Portland State University

PDXScholar

Spring 7-15-2016

\title{
Seismic Behavior and Design of the Linked Column Steel Frame System for Rapid Return to Occupancy
}

Arlindo Pires Lopes

Portland State University

Follow this and additional works at: https://pdxscholar.library.pdx.edu/open_access_etds

Part of the Civil and Environmental Engineering Commons Let us know how access to this document benefits you.

\section{Recommended Citation}

Lopes, Arlindo Pires, "Seismic Behavior and Design of the Linked Column Steel Frame System for Rapid Return to Occupancy" (2016). Dissertations and Theses. Paper 3012.

https://doi.org/10.15760/etd.3007

This Dissertation is brought to you for free and open access. It has been accepted for inclusion in Dissertations and Theses by an authorized administrator of PDXScholar. Please contact us if we can make this document more accessible: pdxscholar@pdx.edu. 
Seismic Behavior and Design of the Linked Column Steel Frame System for

Rapid Return to Occupancy

by

Arlindo Pires Lopes

A dissertation submitted in partial fulfillment of the requirements for the degree of

\author{
Doctor of Philosophy \\ in \\ Civil and Environmental Engineering
}

\author{
Dissertation Committee: \\ Peter Dusicka, Chair \\ Franz Rad \\ Hormoz Zareh \\ Qi-Song $\mathrm{Yu}$
}

Portland State University

2016 


\begin{abstract}
The Linked Column Frame (LCF) is a new brace-free lateral structural steel system intended for rapid return to occupancy performance level. LCF is more resilient under a design level earthquake than the conventional approaches. The structural system consists of moment frames for gravity that combines with closely spaced dual columns (LC) interconnected with bolted links for the lateral system. The LC links are sacrificial and intended to be replaced following a design level earthquake. The centerpiece of this work was a unique full-scale experiment using hybrid testing; a combination of physical test of a critical sub-system tied to a numerical model of the building frame. Hybrid testing allows for full scale study at the system level accounting for the uncertainties via experimental component and having the ability to model more conventional behavior through numerical simulation. The experimental subsystem consisted of a two story LCF frame with a single bay while the remainder of the building was numerically modeled. Two actuators per story were connected to the specimen. The LC links have been designed to be short and plastically shear dominated and the LCF met the design intent of $2.5 \%$ inter-story drift limits. For evaluating the LCF response, hybrid testing was performed for ground motion at three different intensities; $50 \%, 10 \%$ and $2 \%$ probability of exceedence in 50 years for Seattle, Washington ground motions. The system overall had exhibited three distinct performance levels; linearly elastic, rapid return to occupancy where only the replaceable links would yield, and collapse prevention where the gravity beam components also became damaged. Results demonstrated a viable system under cyclic and seismic loading, offering a ductile structural system with the ability to rapidly return to occupancy.
\end{abstract}




\section{Acknowledgements}

I thank God for guiding me through this doctoral process. For God, nothing is impossible.

My time at Portland State University (PSU) has been blessed with challenging course work and research in other fields besides the work presented in this dissertation. I would like to express my sincere gratitude to my advisor, Dr. Peter Dusicka, for his guidance, encouragement, and support during my studies, without which this research would not have been possible. It has been an honor to be his first Ph.D. graduate.

Support from the National Science Foundation via the Network for Earthquake Engineering Simulation (NEES) research under Grant No. 0830414 is greatly appreciated.

My thankfulness also goes to my doctoral committee members, Dr. Franz Rad, Dr. Hormoz Zareh, and Dr. Kent Yu, who have provided educational guidance and incentive through valuable advice regarding my studies.

I would like to thank the Department of Civil and Environmental Engineering at PSU. In particular, Megan Falcone and Ariel Lewis for their hard work.

The iSTAR Laboratory has a wealth of excellent students which have made my time here memorable. Thanks to my officemates for their help and friendliness, especially Max Stephens, Gregory Lewis, Selamawit Mehary, Michael Noddings, Marshall Stokes, Stuart Garth, Ilya Palnikov, and Ramiro Bassaez.

Thanks to the laboratory staff at the University of California, Berkeley for 
their hard work during the experimental program of this research. I am especially grateful to Dr. Shakhzod Takhirov and Dr. Selim Gunay for their invaluable assistance in the experimental program.

I would like especially to thank Mr. and Mrs. Downing. Thanks for being such good friends since we first came to Portland. Thanks to the Bible study group for their continuous pray and spiritual support.

The leave of absence from my home institution Universidade do Estado do Amazonas - UEA necessary to pursue my doctoral degree is gratefully acknowledged. I am thankful to the Dean of Graduate Studies Dr. Jose Luiz Pio, for his help with administrative matters related to my absence.

Sincere gratitude goes to my parents, Dr. Rogerio Lopes and Fatima Pires, who guided me and helped me to be what I am and did provide me with everything I dreamed in my entire life. It may not be possible for me to return their endless love and sacrifice. I would like to thank my father for being understanding and supportive with all the decisions that I have made in my life. Thanks for teaching me how to score a goal in a free penalty kick and how to understand a free body diagram.

My mother who is the strongest and bravest woman I know, has been my biggest fan and at the same time my biggest inspiration, deserves a special mention. Mom, you encouraged and inspired me during all these years. Thank you for always being by my side helping me put things in perspective and move on. Thanks to my big brother Wilson and my little sister Evelyn, for their unconditional love and support in all my endeavors.

Finally, special thanks to my wife, Adriana, for helping me finish this program and making this journey a wonderful and unforgettable experience. I would not have been able to complete this program without her continuous love, patience, and encouragement. Adriana has made countless sacrifices to help me get to this point. For these and many other reasons I will forever be grateful. I dedicate this dissertation to my beautiful wife. 


\section{Table of Contents}

Abstract ........................... i

Acknowledgements ........................ ii

List of Tables . . . . . . . . . . . . . . . . . . . . . . . $\mathrm{x}$

List of Figures . . . . . . . . . . . . . . . . . . xii

$\begin{array}{lll}\text { Chapter } 1 & \text { Introduction } & 1\end{array}$

1.1 Research Motivation ................. 1

1.2 NEESR-II LCF Research Project . . . . . . . . . . . . . . 2

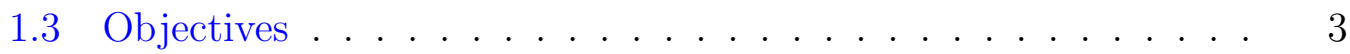

1.4 Contributions to the Field . . . . . . . . . . . . . . . . . . 4

1.5 Dissertation Organization .............. . 4

$\begin{array}{lll}\text { Chapter } 2 & \text { Literature Review } & 7\end{array}$

2.1 Introduction . . . . . . . . . . . . . . . 7

2.2 Moment Resisting Frames . . . . . . . . . . . . . . 8

2.3 Concentrically Braced Frames . . . . . . . . . . . . . . . . . 9

2.4 Eccentrically Braced Frames . . . . . . . . . . . . . . . . 10

2.4.1 Links ..................... . . 11

2.4.2 Link Overstrength . . . . . . . . . . . . . . . 15

2.5 Special Truss Moment Frames . . . . . . . . . . . . . . . . . . . 17

2.6 Other Lateral-Load Resisting Frames . . . . . . . . . . . . . . 18

2.7 Experimental Methods for Structures . . . . . . . . . . . . 22

Chapter 3 Linked Column Steel Frame System 24 
3.1 Introduction . . . . . . . . . . . . . . . . . 24

3.2 Prototype Buildings . . . . . . . . . . . . . . . . . 27

3.3 Drift Sensitivity Analyses _. . . . . . . . . . . . . . . . 29

3.4 Lateral Performance . . . . . . . . . . . . . . . . . . . . 34

3.4 .1 Numerical Model . . . . . . . . . . . . . . . . . . . . . 34

3.4 .2 Lateral Response . . . . . . . . . . . . . . . . . 34

3.4.3 Performance Characteristics . . . . . . . . . . . 36

3.5 Preliminary Design Recommendations . . . . . . . . . . . . 39

Chapter 4 Numerical Analyses Toward Experimental Validation 41

4.1 Introduction . . . . . . . . . . . . . . . . . . . . 41

4.2 Building Geometry . . . . . . . . . . . . . . . . 42

4.3 System Efficiency . . . . . . . . . . . . . . . . . . . . 43

4.4 LCF Building Selection . . . . . . . . . . . . . . . 50

4.5 Earthquake Selection . . . . . . . . . . . . . . . . . 54

4.6 LCF Building Response . . . . . . . . . . . . . . . . . 56

4.6.1 Equivalent Lateral Force Procedure . . . . . . . . . . 57

4.6 .2 Pushover Analysis . . . . . . . . . . . . . . . . . 61

4.6.3 Response History Analysis . . . . . . . . . . . . . . . 64

4.7 Numerical Substructure Component . . . . . . . . . . . . . 66

$\begin{array}{lll}\text { Chapter } 5 & \text { Experimental Program } & 69\end{array}$

5.1 Introduction . . . . . . . . . . . . . . . . . . 69

5.2 Specimen Design . . . . . . . . . . . . . . . . 70

5.2 .1 Specimen Dimensions . . . . . . . . . . . . . . . . 70

5.2 .2 Beams and Columns . . . . . . . . . . . . . . . . . 72

5.2 .3 Shear Links . . . . . . . . . . . . . . . . . 76

5.2 .4 Frame Connections . . . . . . . . . . . . . . . . 81

5.2.5 Column Base Plates and Clevises . . . . . . . . . . . 82

5.2.6 Summary of Design Characteristics . . . . . . . . . 87 
5.3 Experimental Test Setup . . . . . . . . . . . . . . . . . . 88

5.3.1 Construction Sequence . . . . . . . . . . . . . . 88

5.3 .2 Actuators and Brackets . . . . . . . . . . . . . . . 93

5.3 .3 Actuator Spreader Beams _. . . . . . . . . . . . 95

5.3.4 Out-of-Plane Restraint System _ . . . . . . . . . . . 96

5.3.5 Completed Test Setup . . . . . . . . . . . . . . . . 103

5.4 Instrumentation . . . . . . . . . . . . . . . . . . . . 103

5.4 .1 Strain gages . . . . . . . . . . . . . . . . . 105

$5.4 .2 \quad$ LVDTs. . . . . . . . . . . . . . . . . . . . 107

5.4 .3 Load Cells . . . . . . . . . . . . . . . . . . . . . . . . . . 108

5.4.4 String Pots and Accelerometers _. . . . . . . . . . 109

5.4 .5 Cameras and Video . . . . . . . . . . . . . . . . . . . . . 110

5.4 .6 Data Acquisition System . . . . . . . . . . . . . . . . 111

5.5 Cyclic Loading Protocol . . . . . . . . . . . . . . . . . . . . 112

5.6 Hybrid Simulation . . . . . . . . . . . . . . . . . . 115

5.7 Test Sequence . . . . . . . . . . . . . . . . . . . . . . . . . . 119

Chapter 6 LCF System Performance Validation 122

6.1 Introduction . . . . . . . . . . . . . . . . . . . . . . . . 122

6.2 Initial Cyclic Behavior Evaluation . . . . . . . . . . . . . . . 123

6.3 Seismic Response of LCF-5L . . . . . . . . . . . . . . . . . . 129

6.4 Replaceability of Links . . . . . . . . . . . . . . . . . . . . . . 144

6.5 Seismic Response of LCF-3L . . . . . . . . . . . . . . . 147

6.6 Ultimate Cyclic Loading . . . . . . . . . . . . . . . . . . . 153

$\begin{array}{lll}\text { Chapter } 7 & \text { LCF Structural Mechanics } & 159\end{array}$

7.1 Introduction . . . . . . . . . . . . . . . . . . . . . 159

7.2 Cantilever Column . . . . . . . . . . . . . . . . . . 160

7.3 Vierendeel Column . . . . . . . . . . . . . . . . . . . . . 166

7.4 Serviceability . . . . . . . . . . . . . . . . . . . 169 
7.5 Axial Load on Foundation . . . . . . . . . . . . . . . . . 172

7.6 Analytical Examples . . . . . . . . . . . . . . . . . . . 177

$\begin{array}{lll}\text { Chapter } 8 & \text { Design Practice for LCF } & 185\end{array}$

8.1 System Geometry . . . . . . . . . . . . . . . . . . . . . . 185

8.1 .1 Floor Height . . . . . . . . . . . . . . . . . 186

8.1 .2 Span Length . . . . . . . . . . . . . . . . . . . . . 186

8.1 .3 Number of Linked Columns . . . . . . . . . . . . . 186

8.1 .4 Link Length . . . . . . . . . . . . . . . . . 186

8.1.5 Steel Floor Systems . . . . . . . . . . . . . . . . . 187

8.2 Loading Conditions . . . . . . . . . . . . . . . . . . . . 187

8.3 Proportion Structural Members . . . . . . . . . . . . . . 188

8.3 .1 Linked Columns . . . . . . . . . . . . . . . . . . 188

$8.3 .2 \quad$ Links . . . . . . . . . . . . . . . . . . . . . . . . . 189

8.3.3 Gravity Beams and Gravity Columns . . . . . . . . . . 189

8.4 Strong Column and Weak Beam Design . . . . . . . . . . . . 190

8.5 Serviceability and Drift . . . . . . . . . . . . . . . . . 192

8.6 Pushover Analysis . . . . . . . . . . . . . . . . . . . . . 193

8.7 Performance Levels . . . . . . . . . . . . . . . . . . . . . . . 194

8.8 Design Example . . . . . . . . . . . . . . . . . . . . 196

$\begin{array}{lll}\text { Chapter } 9 & \text { Seismic Performance Assessment } & 217\end{array}$

9.1 Introduction . . . . . . . . . . . . . . . . . . . 217

9.2 Analysis Procedure Selection . . . . . . . . . . . . . . . . 218

9.3 Linear Static Procedure . . . . . . . . . . . . . . . . . . . 219

9.4 Load Combination . . . . . . . . . . . . . . . . . 225

9.5 Strength of LCF components . . . . . . . . . . . . . . 226

9.5 .1 Beams . . . . . . . . . . . . . . . 226

9.5.2 Replaceable Links . . . . . . . . . . . . . . . . . . . 229

9.5 .3 Columns . . . . . . . . . . . . . . . . . . . . . 230 
9.6 Acceptance Criteria . . . . . . . . . . . . . . . . . . . . 233

9.7 Assessment Example . . . . . . . . . . . . . . . . . . . 236

9.7 .1 Beams . . . . . . . . . . . . . . . . . . 239

9.7.1.1 Flexure Demand . . . . . . . . . . . . . . . 239

9.7.1.2 Flexure Strength . . . . . . . . . . . . . . 239

9.7.1.3 Acceptance Criteria Check . . . . . . . . . . . . 241

9.7 .2 Replaceable Links . . . . . . . . . . . . . . . . . . . . 242

9.7.2.1 Shear Demand . . . . . . . . . . . . . . . . . 242

9.7.2.2 Shear Strength . . . . . . . . . . . . . . . 243

9.7.2.3 Acceptance Criteria Check . . . . . . . . . . . . 243

9.7 .3 Columns . . . . . . . . . . . . . . . . . . . . . 244

9.7.3.1 Axial Demand . . . . . . . . . . . . . . . . 244

9.7.3.2 Axial Strength . . . . . . . . . . . . . . 245

9.7.3.3 Flexure Demand . . . . . . . . . . . . . . . 246

9.7.3.4 Flexural Strength . . . . . . . . . . . . . 246

9.7.3.5 Acceptance Criteria Check . . . . . . . . . . . . 248

9.8 Observations . . . . . . . . . . . . . . . . . . . . . . . . 249

Chapter 10 Design Recommendations 251

10.1 Seismic Performance Factors . . . . . . . . . . . . . . . . . . 251

10.2 Proposed LCF Section for the Seismic Provisions . . . . . . . 252

10.3 Horizontal Diaphragm Load Transfer . . . . . . . . . . . . . 259

10.4 Replaceable Link . . . . . . . . . . . . . . . . . . . . . 260

10.5 Foundation . . . . . . . . . . . . . . . . 262

$\begin{array}{ll}\text { Chapter } 11 \text { Conclusions and Future Studies } & 267\end{array}$

11.1 Conclusions . . . . . . . . . . . . . . . . . . . 267

11.2 Suggestions for Future Studies . . . . . . . . . . . . . . 272

$\begin{array}{ll}\text { References } & 274\end{array}$

viii 


\section{List of Tables}

Table 3.1 Structural members for SMRF buildings. . . . . . . . . 28

Table 3.2 Structural members for LCF buildings . . . . . . . . . . 32

Table 3.3 LC links dimensions $(\mathrm{mm}) \ldots \ldots \ldots$

Table 3.4 LCF system lateral parameters . . . . . . . . . . . 38

Table 4.1 Structural members for LCF buildings. . . . . . . . . . . 45

Table 4.2 Structural members for LCF buildings. . . . . . . . . . 46

Table 4.3 LCF system lateral parameters. . . . . . . . . . . . 47

Table 4.4 Details of Seattle ground motions. . . . . . . . . . 55

Table 4.5 Details of Seattle seismic forces. . . . . . . . . . . 60

Table 4.6 Drift story check for the 2-story \& 4-bay LCF systems. . . 61

Table 4.7 LCF system lateral parameters . . . . . . . . . . . . 63

Table 4.8 Expected internal forces during the experiment. . . . . . . 65

Table 5.1 FEMA 461 parameters. . . . . . . . . . . . . . . . . 114

Table 5.2 Test sequence on the LCF test assembly. . . . . . . . . 120

Table 6.1 Panel Zone $\gamma_{\max }$ and location. . . . . . . . . . . . . 142

Table 7.1 Partial and total displacements for different story levels. . 164

Table 7.2 Linked column axial force at foundation level. . . . . . . . 173

Table 7.3 Linked Column Member Sizing for a 2-story LCF Building. 179

Table 7.4 Linked Column Member Sizing for a 3-story LCF Building. 181

Table 7.5 Linked Column Member Sizing for a 6-story LCF Building. 182

Table 7.6 Summary of the member sizing. . . . . . . . . . . . 183 
Table 8.1 Deflection limit for beams. . . . . . . . . . . . . . . . 192

Table 8.2 Seismic design parameters for the LCF sample building. 201

Table 8.3 Lateral Force Distribution Calculations for the LCF sample building. . . . . . . . . . . . . . . . . . . . . 202

Table 8.4 First model sizing for a 4-story LCF Building. . . . . . . . 204

Table 8.5 First model drift analysis for the LCF sample building. . . 208

Table 8.6 Member sizing for the LCF sample building. . . . . . . . . 210

Table 8.7 Third model link dimensions (in) . . . . . . . . . . . . 210

Table 8.8 Second model drift analysis for the LCF sample building. 211

Table 8.9 Third model drift analysis for the LCF sample building. . 211

Table 8.10 Performance parameters for the LCF sample building. . . 215

Table 9.1 Seismic design parameters for the LSP on the LCF 2-story building. . . . . . . . . . . . . . . . . . . . 238

Table 9.2 DCR values for gravity beams on the LCF 2-story building. 242

Table 9.3 DCR values for shear links on the LCF 2-story building. . 244

Table 9.4 DCR values for columns subjected to compression forces on the LCF 2-story building. . . . . . . . . . . . . . . . . 249

Table 9.5 DCR values for columns subjected to tension forces on the LCF 2-story building. . . . . . . . . . . . . . . . . . 249

Table 10.1 Seismic performance factors for LCF buildings. . . . . . . 252 


\section{List of Figures}

Figure 2.1 Moment resisting frame. . . . . . . . . . . . . 8

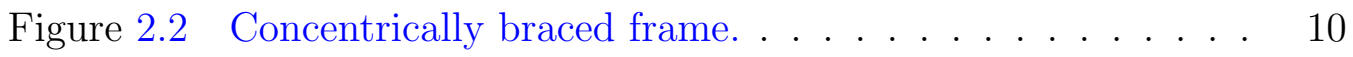

Figure 2.3 Eccentrically braced frame. . . . . . . . . . . . . 11

Figure 2.4 Rendering of the new SFOBB suspension signature span. 12

Figure 2.5 Pushover analysis of the single tower. . . . . . . . . . 13

Figure 2.6 Ultimate failure of link L225. . . . . . . . . . . . . 13

Figure 2.7 Experimental setup at iSTAR laboratory. . . . . . . . . . 14

Figure 2.8 Strain distribution of link 25DBase. . . . . . . . . . 16

Figure 2.9 STMF with a single panel Vierendel ductile segment. . . 18

Figure 2.10 Special steel plate shear wall. . . . . . . . . . . . . . . 19

Figure 2.11 Buckling retrained braced frame. . . . . . . . . . 20

Figure 2.12 Preliminary LCF experimental test setup. . . . . . . . 22

Figure 3.1 Elevation of a 3 -story LCF building. . . . . . . . . 26

Figure 3.2 Idealized lateral response. . . . . . . . . . . . . . . 26

Figure 3.3 Inter-story drift sensitivity analyses. . . . . . . . . . 30

Figure 3.4 Pushover comparisons. . . . . . . . . . . . . . . . . . 36

Figure 3.5 Modified model for a 3 -story LCF building. . . . . . . . . 37

Figure 4.1 Plan view of the LCF building considered in the numerical analyses. . . . . . . . . . . . . . . . . . . 43

Figure 4.2 Different boundary conditions for links. . . . . . . . . 45

Figure 4.3 Pushover response for different LCF systems. . . . . . . . 46 
Figure 4.4 Element contribution to LCF-B-ISL and LCF-W-ISL sys-

tems. . . . . . . . . . . . . . . . . 49

Figure 4.5 Element contribution to LCF-B-L and LCF-W-L systems. 50

Figure 4.6 Numerical model for specimen LCF-5L. . . . . . . . . . 52

Figure 4.7 Numerical model for specimen LCF-3L. . . . . . . . . . 53

Figure 4.8 Ground motions used in the dynamic analyses. . . . . . . 55

Figure 4.9 Acceleration time history used in the dynamic analyses. . 56

Figure 4.10 Seismic forces used in the ELF procedure. . . . . . . . 60

Figure 4.11 Deformed shape for the LCF-5L system. . . . . . . . . 61

Figure 4.12 Estimate of the lateral response for LCF-5L system. . . . 63

Figure 4.13 Base Shear vs Drift for LCF-3L system. . . . . . . . . . 65

Figure 4.14 Numerical substructure component. . . . . . . . . . . . 67

Figure 5.1 Elevation of the LCF specimens. . . . . . . . . . 71

Figure 5.2 Free-body diagram of a link. . . . . . . . . . . . . 76

Figure 5.3 Shear link detailing. . . . . . . . . . . . . . . . . . . . 79

Figure 5.4 Shear link connection. . . . . . . . . . . . . . 80

Figure 5.5 Extended-end plate moment connection. . . . . . . . . 83

Figure 5.6 Double angle shear connection. . . . . . . . . . . 84

Figure 5.7 (a) Linked column base plate; (b) Gravity column base plate. . . . . . . . . . . . . . . . . 85

Figure 5.8 Dimensional requirements for pin-connected members. . . 86

Figure 5.9 Linked column clevis. . . . . . . . . . . . . . . . . 86

Figure 5.10 Gravity column clevis. . . . . . . . . . . . . . 87

Figure 5.11 (a) LC floor beam; (b) Actuator bracket. . . . . . . . . . 89

Figure 5.12 Linked columns of LCF-5L specimen being installed on the LC floor beam. . . . . . . . . . . . . . . . . . . . 90

Figure 5.13 GC floor beam. . . . . . . . . . . . . . . . . 91

Figure 5.14 Sliding mechanism B at the first floor level. . . . . . . . . 92 
Figure 5.15 Second floor T-section beam being lifted. . . . . . . . . . 93

Figure 5.16 Static actuators used in the LCF system experimental test. 94

Figure 5.17 Dynamic actuators used in the LCF system experimental

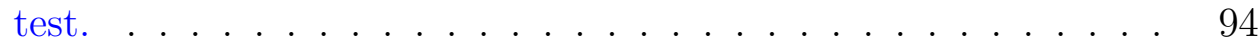

Figure 5.18 Elevation view of the actuator brackets. . . . . . . . . . 95

Figure 5.19 Actuator brackets installed on the reaction wall. . . . . . 96

Figure 5.20 Actuator spreader beam details. . . . . . . . . . . . 97

Figure 5.21 Actuator spreader beam located at the second floor. . . . 97

Figure 5.22 3D view of the out-of-plane restraint system. . . . . . . . 98

Figure 5.23 C-A and C-B connectors located at the second floor level. 99

Figure 5.24 Connector C-B details. . . . . . . . . . . . . . . . . . . . 100

Figure 5.25 Connector C-C details. . . . . . . . . . . . . . . . . . . . 101

Figure 5.26 Sliding mechanism A at first floor level. . . . . . . . . . . 101

Figure 5.27 Sliding mechanism B at first floor level. . . . . . . . . . . 102

Figure 5.28 Completed test setup for the LCF-5L specimen before testing. . . . . . . . . . . . . . . . . 104

Figure 5.29 Strain gage layout used on LCF test assembly. . . . . . . 106

Figure 5.30 Strain gage protection. . . . . . . . . . . . . . . 107

Figure 5.31 LVDT layout used on LCF test assembly. . . . . . . . . . 108

Figure 5.32 Load cell already installed and load cell cross-section. . . 109

Figure 5.33 String pot at the first story level. . . . . . . . . . . . . . 109

Figure 5.34 Accelerometer used to obtain rotation. . . . . . . . . . . 110

Figure 5.35 String pots and accelerometers layout used on LCF test assembly. ........................ . . 111

Figure 5.36 Cameras and laser scanner used on LCF test assembly. 112

Figure 5.37 Switch boxes and cable extension. . . . . . . . . . . 113

Figure 5.38 Intermediate gage device attached to the LCF specimen. 113

Figure 5.39 Displacement-controlled loading history used on LCF test assembly. . . . . . . . . . . . . . . . . 115

xiv 
Figure 5.40 Hybrid simulation setup used on LCF-5L test assembly. . 117

Figure 5.41 Interacting components of a hybrid simulation test. . . . 119

Figure 6.1 Friction test: Base shear versus overall drift. . . . . . . . 123

Figure 6.2 Base shear vs LC1 base rotation for LCF-5L specimen. . 124

Figure 6.3 Base shear vs GC base rotation for LCF-5L specimen. . . 125

Figure 6.4 Shear on linked columns vs Link 1 rotation through LVDT.126

Figure 6.5 Base shear vs moment connection rotation for LCF-5L specimen. . . . . . . . . . . . . . . . 127

Figure 6.6 Base shear vs shear connection rotation for LCF-5L specimen. . . . . . . . . . . . . . . . . . . . . . . . . . 128

Figure 6.7 LCF system overstrength. . . . . . . . . . . . . . 129

Figure 6.8 Lump masses for hybrid simulation test. . . . . . . . 130

Figure 6.9 Stiffness test results for LCF-5L specimen. . . . . . . . . 131

Figure 6.10 Axial loads along LC1 height for LCF-5L specimen . . . 134

Figure 6.11 Axial loads along LC2 height for LCF-5L specimen . . . 135

Figure 6.12 Moments at LC1 for LCF-5L specimen. . . . . . . . . . 136

Figure 6.13 Moments at LC2 for LCF-5L specimen. . . . . . . . . . . 137

Figure 6.14 Moments at GB1 for LCF-5L specimen. . . . . . . . . 138

Figure 6.15 Moments at GB2 for LCF-5L specimen. . . . . . . . . . 139

Figure 6.16 Gravity beam strain distribution for LCF-5L specimen. . 140

Figure 6.17 Link behavior along column height: LCF 5 links . . . . . 141

Figure 6.18 Damage in structural members at 1.4\% drift. . . . . . . . 142

Figure 6.19 Free body diagram to calculate shear on links. . . . . . . 143

Figure 6.20 Shear on links vs levels of earthquake for LCF-5L specimen.144

Figure 6.21 Experimental test setup after hybrid testing for LCF-5L specimen. . . . . . . . . . . . . . . . . . 145

Figure 6.22 Replaceability of shear link at mid-height of second floor. 146 Figure 6.23 Deformed shear links after testing the LCF-5L specimen. 147 
Figure 6.24 fig:LCF hybrid model. . . . . . . . . . . . . . . . . . 148

Figure 6.25 Base shear versus drift. . . . . . . . . . . . . . . . . . 149

Figure 6.26 Axial loads along LC1 height for LCF-3L specimen . . . 150

Figure 6.27 Axial loads along LC2 height for LCF-3L specimen . . . 151

Figure 6.28 Shear on links vs levels of earthquake for LCF-3L specimen.152

Figure 6.29 Shear deformation versus drift for LCF-3L specimen during RR earthquake. . . . . . . . . . . . . . . . . . . 153

Figure 6.30 Shear deformation versus drift for LCF-3L specimen during $\mathrm{CP}$ earthquake. . . . . . . . . . . . . . . . . . . 154

Figure 6.31 Progression of damage of Link A for LCF-3L specimen. . 155

Figure 6.32 Flange strain distribution of gravity beams for LCF-3L specimen. . . . . . . . . . . . . . . . . 156

Figure 6.33 Base shear versus drift; Link shear deformation versus drift hysteresis. . . . . . . . . . . . . . . . . . 157

Figure 6.34 Damage in structural members at 5\% drift. . . . . . . . 157

Figure 6.35 Friction test setup. . . . . . . . . . . . . . . 158

Figure 7.1 Cantilever column of a 2-story LCF building. . . . . . . . 161

Figure 7.2 Cantilever column of a 3-story LCF building. . . . . . . . 163

Figure 7.3 Cantilever column of a 6-story LCF building. . . . . . . 165

Figure 7.4 Total displacement versus number of stories. . . . . . 166

Figure 7.5 Vierendeel column. . . . . . . . . . . . . . . . . . . 167

Figure 7.6 Gravity beam model for a LCF building. . . . . . . . . 170

Figure 7.7 LCF 2-story linked column. . . . . . . . . . . . . . 173

Figure 7.8 Foundation reaction parameters versus number of stories. 174

Figure 7.9 Plastic shear links on linked columns. . . . . . . . . . . 176

Figure 7.10 2-story LCF building layout. . . . . . . . . . . . 177

Figure 7.11 Cantilever model for a 2-story LCF building. . . . . . . . 178

Figure 7.123 -story LCF building layout. . . . . . . . . . . . . . 180 
Figure 7.13 Cantilever model for a 3-story LCF building. . . . . . . . 180

Figure 7.14 6-story LCF building layout. . . . . . . . . . . . . . . . . 182

Figure 7.15 Cantilever model for a 6-story LCF building. . . . . . . . 183

Figure 8.1 (a) story mechanism and $(b)$ beam mechanism. . . . . . . 191

Figure 8.2 LCF building sequence of yielding: links and then gravity beams. ......................... 193

Figure 8.3 Limit states of a LCF building. . . . . . . . . . . . . . . 195

Figure 8.4 Linked Column Frame Flowchart. . . . . . . . . . . . . . 198

Figure 8.5 Plan view of LCF sample building. . . . . . . . . . . . . 199

Figure 8.6 Elevation view of LCF sample building. . . . . . . . . . . 200

Figure 8.7 4-story LCF building layout. . . . . . . . . . . . . . . . . 203

Figure 8.8 Cantilever model for a 4-story LCF building. . . . . . . . 203

Figure 8.9 First model strong column and weak beam design calcu-

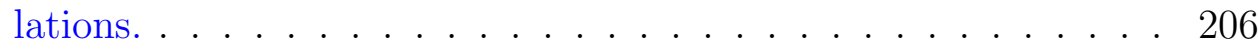

Figure 8.10 LCF substructure labeling. . . . . . . . . . . . . . . . . . 209

Figure 8.11 Pushover curves for the LCF sample building. . . . . . . 213

Figure 9.1 Flowchart for the LCF linear static procedure. . . . . . . 220

Figure 9.2 General Horizontal Response Spectrum. . . . . . . . . . . 222

Figure 9.3 LCF pseudo seismic force flowchart for linear static pro-

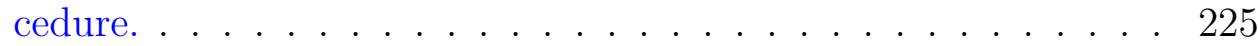

Figure 9.4 LCF acceptance criteria flowchart. . . . . . . . . . . . . 234

Figure 9.5 Seismic assessment for structural members on the LCF 2-story building. . . . . . . . . . . . . . . . 237

Figure 9.6 LCF 2-story building labeling for seismic assessment. . . 237

Figure 10.1 Horizontal diaphragm load transfer. . . . . . . . . . . . . 260

Figure 10.2 Replaceable shear link. . . . . . . . . . . . . . . . . 261

Figure 10.3 Pile cap plan view. . . . . . . . . . . . . . . . . . . 263 
Figure 10.4 Pile cap lateral view without anchor bolts. . . . . . . . . 264

Figure 10.5 Base plate pan view for the pile cap. . . . . . . . . . . 265

Figure 10.6 Pile cap lateral view with anchor bolts. . . . . . . . . . . 266

Figure 10.7 Pile cap front view with anchor bolts. . . . . . . . . . 266 


\section{Chapter 1}

\section{Introduction}

\subsection{Research Motivation}

Seismic design approach is generally based on elastic structural behavior and accounts for the inelastic behavior indirectly (i.e., force modification factor, occupancy importance factor, and deflection amplification factor). According to Ghobarah (2001) although buildings designed to current codes performed well during recent earthquakes from a life safety perspective, the level of damage to structures, economic loss due to loss of use, and cost of repair were unexpectedly high.

Performance-based seismic design (FEMA 445, 2006) is a more general design philosophy in which the design criteria are expressed in terms of achieving stated performance objectives when the structure is subjected to specific levels of seismic hazard. Two predominant methods, the coefficient method (FEMA 356, 2000) and the capacity-spectrum method (FEMA 440, 2005) are most often used in current U.S. practice. Both approaches use nonlinear static analysis (pushover analysis) to estimate seismic demands and capacities.

New structural systems are being developed with emphasis on immediate occupancy following a design level earthquake. Example of these systems include development of damage free beam-column connection, either through post tensioning (Ricles et. al., 2001) or friction based resistance (Khoo et. al., 2011). An alternative approach to immediate occupancy performance level is to design 
for damage in non-gravity members that could be replaced. Such structural systems would be capable of rapid repair that would facilitate return to occupancy following a seismic event. For example, buckling restrained brace frames exhibit this characteristic.

A non-braced structural system was developed at Portland State University (PSU) and is referred to as the Linked Column Frame (LCF) system. The LCF aims to address the rapid return to occupancy design performance while maintaining the architectural advantages of non-braced steel frame construction.

\subsection{NEESR-II LCF Research Project}

A large research project funded by the National Science Foundation (NSF) under the umbrella of the George E. Brown Network for Earthquake Engineering Simulation Research (NEESR-II) was undertaken. This project was named "Toward Rapid Return to Occupancy in Unbraced Steel Frames" and the overall vision of this project was to develop a lateral load-resisting system for unbraced steel frames capable of achieving specific target performance levels. PSU was the leading institution and the project also had collaborators from the University of Washington, who conducted system level computer simulations, and California State University in Los Angeles, who helped with some of the preliminary work and outreach. The research began with numerical and experimental investigation of wide-flange links for use in the LCF system utilizing plate bolted connections (Lewis, 2010) followed by studies on composite sandwich links for the LCF system (Stephens, 2011). Concurrent with these tests, two independent numerical modeling and design on the LCF system level were performed by Malakoutian (2012) and Lopes et. al. $\left(2012^{a}\right)$ in which the latter one was chosen toward LCF system experimental tests. 


\subsection{Objectives}

Building upon the research described in Section (1.2), full-scale LCF tests at the NEES structural laboratory of the University of California at Berkeley (nees@berkeley) were conducted culminating in the development of design recommendations for LCF systems based on the observations and data gathered during the experimental and numerical tasks.

These full-scale tests were the first tests ever conducted on LCF systems using either cyclic or hybrid simulation, which is a combination of physical test of a critical experimental substructure tied to a numerical substructure component of the building. The main objectives of this dissertation were as follows:

1. To develop a numerical model toward experimental validation through drift sensitivity analysis and lateral performance.

2. To investigate different boundary conditions on the system and use of built-up sections for the replaceable links.

3. To develop a numerical substructure component model in order to proceed with the hybrid simulation tests.

4. To prepare, execute and report findings of a unique full-scale LCF experiment using cyclic and hybrid simulation tests.

5. To experimentally validate the LCF system performance.

6. To experimentally find the Overstrength coefficient, $\Omega_{0}$, which is a seismic performance factor.

7. To develop closed-form equations via structural mechanics for estimating the lateral stiffness of LCF buildings and axial load on foundations.

8. To provide general guidelines for preliminary LCF design. 
9. To study through seismic performance assessment the fit of the LCF system into performance based design guidelines.

10. To propose additions to the current seismic code provisions to incorporate the design of LCF buildings.

11. To provide structural detailing for horizontal diaphragm and linked column foundations.

\subsection{Contributions to the Field}

This research was the first methodical and comprehensive evaluation of LCF buildings, including development, numerical analyses, experimental investigation, analytical studies, and bridge between academic and practice. Ultimately, the LCF system performance validation was done by testing full-scale specimens through cyclic and hybrid simulation investigations leading to design guidelines for the LCF system in order to obtain multiple performance objectives simultaneously. This dissertation is intended to define a state-of-the-art method for

evaluation of LCF buildings, and therefore provide guidance to the seismic design of such structures in moderate-to-high seismicity regions.

\subsection{Dissertation Organization}

The dissertation is organized in 11 chapters, appendices and a list of references as follows:

- Chapter 1 discusses the motivation of the study, NEESR-II LCF research project, objectives, and contributions to the field.

- Chapter 2 presents a literature review and includes previous research on lateral-load resisting frame systems, links and experimental methods. 
- Chapter 3 describes the LCF system through drift sensitivity analysis and lateral performance, illustrating that a viable non-braced frame alternative system is possible to be implemented. Also, an economic evaluation comparison between LCF and Special Moment Resisting Frame (SMRF) was made. Structural steel weight was calculated for each of the systems as an indicator of cost of the structure.

- Chapter 4 considers numerical analyses toward experimental validation. Changing boundary conditions and using built-up sections for the replaceable links have shown an increase of LCF system's efficiency. Non-linear pushover analyses were used to validate the system ability to achieve the rapid return to occupancy performance level.

- Chapter 5 gives a description of the experimental program that was conducted at nees@berkeley, using cyclic and hybrid simulation tests. The centerpiece of this work was a unique full-scale experiment consisted of a two story LCF building with a single bay, while the remainder of the building was numerically modeled.

- Chapter 6 contains the LCF system performance validation via the discussion of the results of the experiments. For evaluating the LCF structural response, FEMA 461 was used as the loading protocol for the cyclic testing, whereas ground motions at three different intensities; 50\%, 10\% and $2 \%$ probability of exceedance in 50 years were used for the hybrid testing. The system overall had exhibited three distinct performance levels: linearly elastic, rapid return to occupancy where only the replaceable links yielded, and collapse prevention where the gravity beam components also became damaged.

- Chapter 7 deals with principles of structural mechanics and their applications to the analysis of LCF systems and components. Most importantly, 
cantilever and Vierendeel approaches applied to LCF buildings were developed leading to closed-form equations that may be used to select the geometric properties not only for the closely spaced dual columns but also the replaceable links, meeting the design inter-story drift limits. In addition to estimating the lateral stiffness of LCF buildings, closed-form equations were also developed to size gravity beam members as well as to estimate the axial load on foundations.

- Chapter 8 provides general guidelines for preliminary LCF design that results in configurations where linked columns and replaceable links are likely to satisfy capacity design requirements. A step-by-step design procedure is illustrated through an example of a 4-story LCF building.

- Chapter 9 shows the results of a structural seismic performance assessment into performance based design guidelines. The seismic performance assessment was conducted using a linear analysis procedure and assessment calculations for beams, replaceable links and columns are presented.

- Chapter 10 proposes structural detailing for horizontal diaphragm and linked column foundations; and also proposes additions to the current seismic code provisions to incorporate the design of LCF buildings, providing the basis of design, the requirements for analysis, and the requirements for the system, members and connections.

- Chapter 11 summarizes the main conclusions and provides recommendations for future studies. 


\section{Chapter 2}

\section{Literature Review}

\section{$2.1 \quad$ Introduction}

Current approaches to structural steel building design for extreme seismic events typically include forms of ductile structural systems contained in the AISC Seismic Provisions. Most of these structural systems utilize gravity load-carrying members to also resist lateral loads. However, inelastic behavior is usually directly related to structural damage, which in the conventional systems typically results in damage to the gravity load-carrying members even for smaller than design level events.

The LCF system incorporates aspects of conventional systems such as Moment Resisting Frames (MRFs) and Eccentrically Braced Frames (EBFs), but combines them to achieve system performance that can be designed to obtain multiple performance objectives simultaneously.

Relevant literature related to conventional system-level studies and other lateral-load resisting frames is examined first, followed by information on ex-

perimental methods for structures. Due to extensive literature that exists on these topics, only few references used to guide the overall scope of this research are discussed here. Additional references are presented throughout the dissertation. 


\subsection{Moment Resisting Frames}

MRFs are, in their simplest form, linear assemblages of beams and columns, with the beams rigidly connected to the columns as shown in Figure (2.1). To achieve economic designs while providing life-safety to the occupants, modern seismic design principles take advantage of the ductility of buildings and design structures for only a fraction of the expected elastic lateral load. In a steel MRF, plastic hinges develop near the beam-to-column connections during large seismic events. These plastic hinges act as ductile fuses, dissipating energy through stable hysteretic behavior while limiting forces transmitted to other components of the structure.

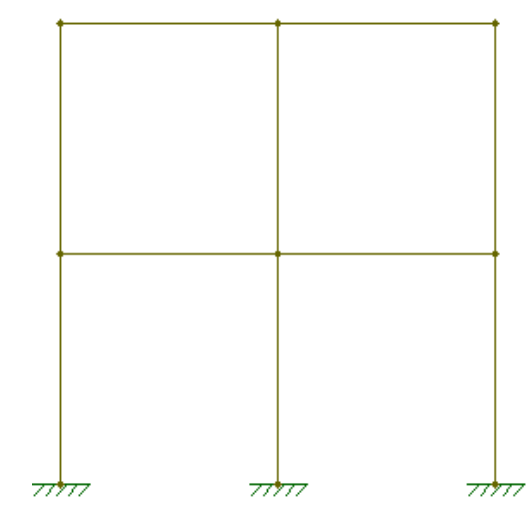

Figure 2.1: Moment resisting frame.

MRFs with traditional welded flange and bolted web connections were believed to be very ductile systems and were extensively used between the 1960s and the early 1990s. This belief was put into question after failures during the Northridge earthquake (Bruneau et. al., 1998), in many cases without any signs of plastic deformation in the beam. As a result of these failures, different schemes were developed to improve the connection performance. Depending on the connection details, the system is classified as ordinary (OMF), intermediate 
(IMF) or special moment frame (SMF) with the latter being best suited for areas of high seismicity.

OMFs are expected to sustain minimal ineslatic deformation in their members and connections when subjected to seismic forces, and it is assumed to have an interstory drift of 0.01 radians and should remain mostly elastic. IMFs are expected to endure limited inelastic deformations in their members and connections when subjected to seismic forces, and the required interstory drift is a minimum of 0.02 radians. Finally, SMFs are expected to undergo significant inelastic deformation when subjected to seismic forces, and the required interstory drift is a minimum of 0.04 radians. Usually, MRFs tend to have heavy member sections and higher story drift compared to other lateral load resisting systems in order to control the design drift.

A conventional MRF is designed to yield and form plastic hinges with associated damages in beams and columns. These damages can result in significant repair costs. In the case of SMF, rapid return to occupancy is not easily achievable since the damage is in the gravity load-carrying beams and, sometimes, in the columns.

\subsection{Concentrically Braced Frames}

Concentrically Braced Frames (CBFs) do not fit in the scope of non-braced structural systems, and also suffer from similar shortcomings when considering return to occupancy despite their ability to provide stiff and ductile response. 
To achieve appropriate strength and ductile response, diagonal braces must be specifically designed to sustain plastic deformation and dissipate hysteretic energy in a stable manner through successive cycles of buckling in compression and yielding in tension (Tremblay \& Tirca, 2003). Schematic of a CBF is shown in Figure (2.2).

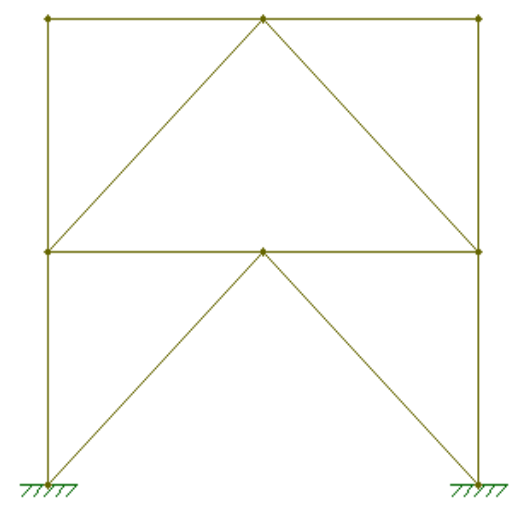

Figure 2.2: Concentrically braced frame.

\subsection{Eccentrically Braced Frames}

Eccentrically Braced Frames (EBFs) are very efficient structures for resisting earthquakes as they combine the ductility of MRFs and the stiffness associated with braced frames. An EBF is a frame system in wich the axial forces induced in the braces are transferred either to a column or to another brace through shear and bending in a small segment of the beam called link. Typical EBF geometry is shown in Figure (2.3). EBFs depend on the inelasticity of specially designed links to provide ductility and to dissipate energy during earthquakes. With increasing emphasis on performance based design, the link's replaceability 
becomes a desired quality. Researchers have begun to examine the possibility of using a bolted link design so that after a seismic event the damaged sections could be replaced (Stratan \& Dubina, 2004) and (Mansour, 2010). Bolted links would also allow for cost effective designs of buildings located in lower seismic regions (Hines, 2009). Unfortunately, EBFs do not fit in the scope of non-braced structural systems.

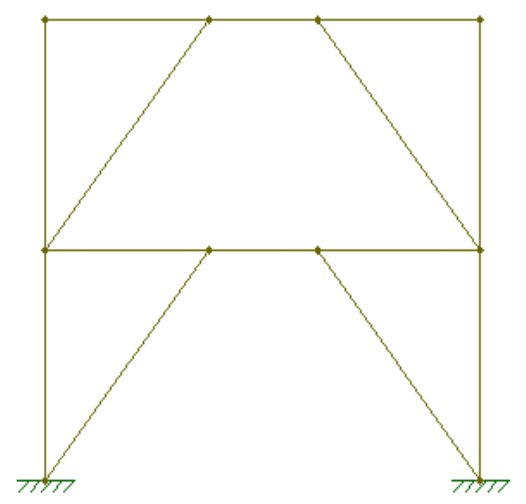

Figure 2.3: Eccentrically braced frame.

\subsubsection{Links}

Links have been used in EBFs to dissipate earthquake energy via large inelastic deformations. They are classified into three types: short, intermediate and long links, depending on the structural and geometric properties (ANSI/AISC 341, 2010). According to Chao et. al. (2006) when architectural constraints permit, short links which dissipate energy primarily through inelastic shear distortion are preferred to longer links that dissipate energy through plastic hinge rotation. Within the scope of this dissertation, replaceable links were 
adopted to interconnect the dual columns on the LCF system.

The idea behind the LCF system was based on recent developments in long span bridge design and applied to building construction. Nader (2000) designed the new San Francisco Oakland Bay Bridge (SFOBB) and one of their approaches to have a clearly defined plastic mechanism for response to lateral loads was to provide replaceable shear links between the tower shafts which would yield in the event of a major earthquake with a clear failure sequence. Figure (2.4) shows the rendering of the new SFOBB suspension signature span and Figure (2.5) shows the effects of using shear links on the lateral behavior of the tower.

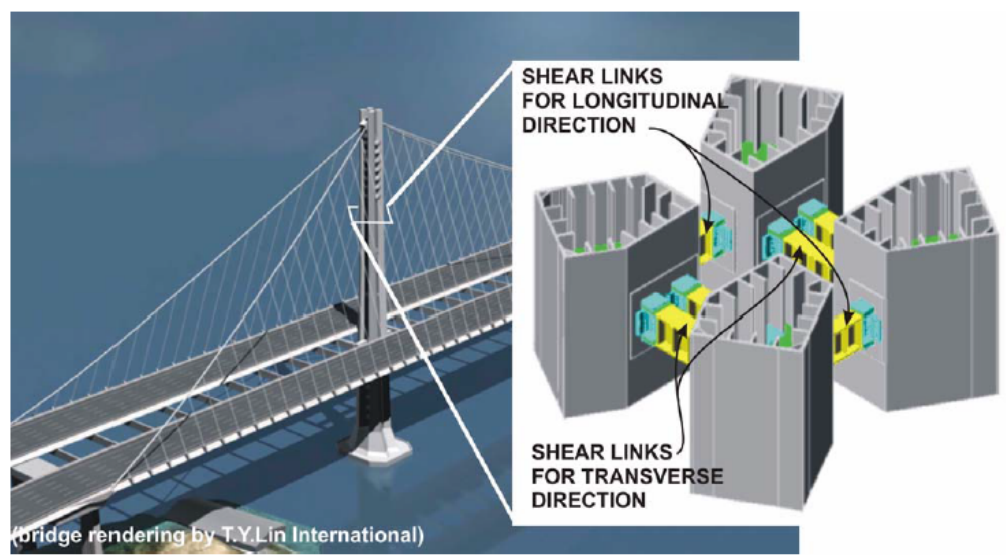

Figure 2.4: Rendering of the new SFOBB suspension signature span.

Dusicka (2004) investigated the inelastic behavior of built-up shear links for seismic protection of bridges through the use of large-scale experiments, material investigation and numerical analyses. Built-up shear links were shown to be effective hysteretic energy dissipators. Figure (2.6) shows the ultimate 


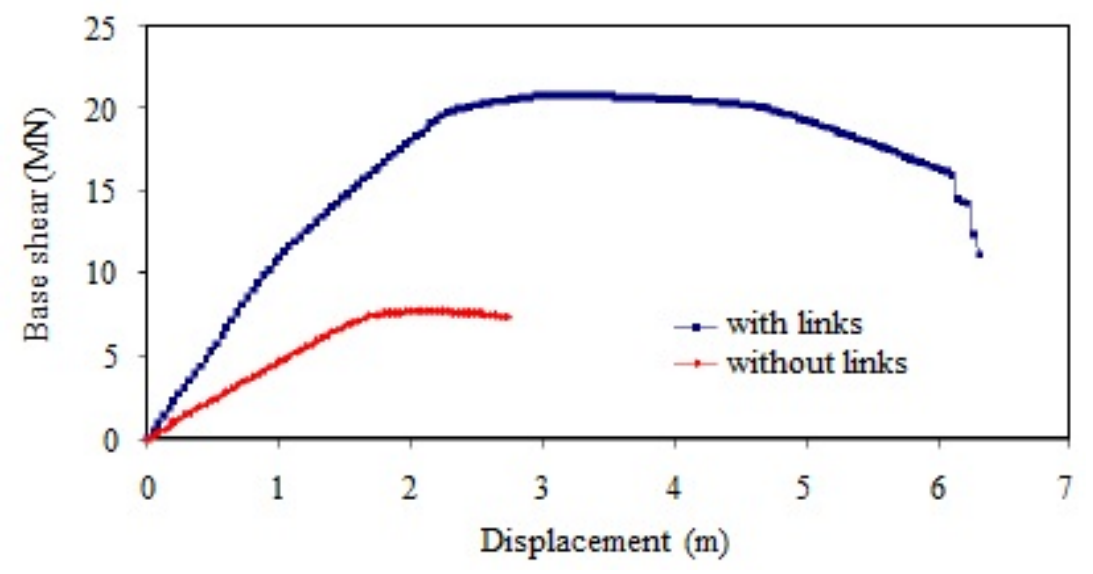

Figure 2.5: Pushover analysis of the single tower.

failure of one of the links tested.

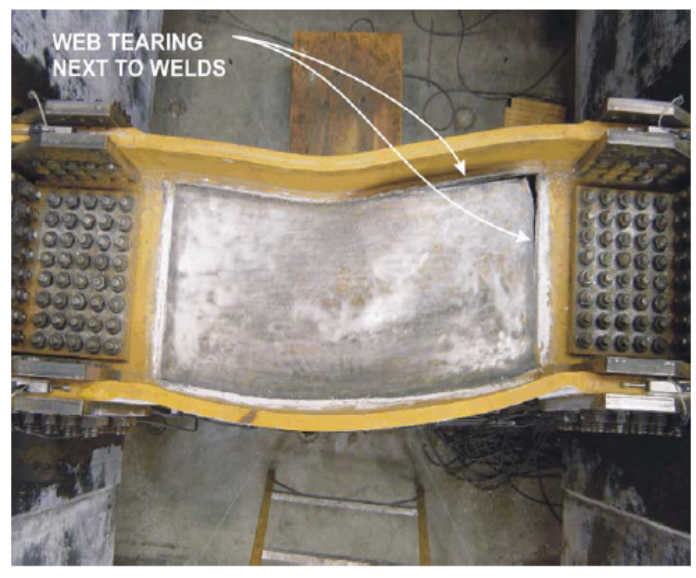

Figure 2.6: Ultimate failure of link L225.

In current lateral systems the shear link is an integral component of the frame system. After an extreme event, retrofit time and costs are increased due to the difficulty in removal and replacement of the damaged link. The LCF system takes an innovative step forward by separating the shear link from the framing system, acting as a seismic dissipater. In addition, the link can now be easily replaced and with less disruption after extreme events. 
Lewis (2010) studied replaceable link connections with the intent of limiting plastic strain at the link-to-end plate connection and thereby minimizing undesirable failure modes. A variety of end stiffener configurations were first investigated through numerical analysis and then experimentally tested at PSU.
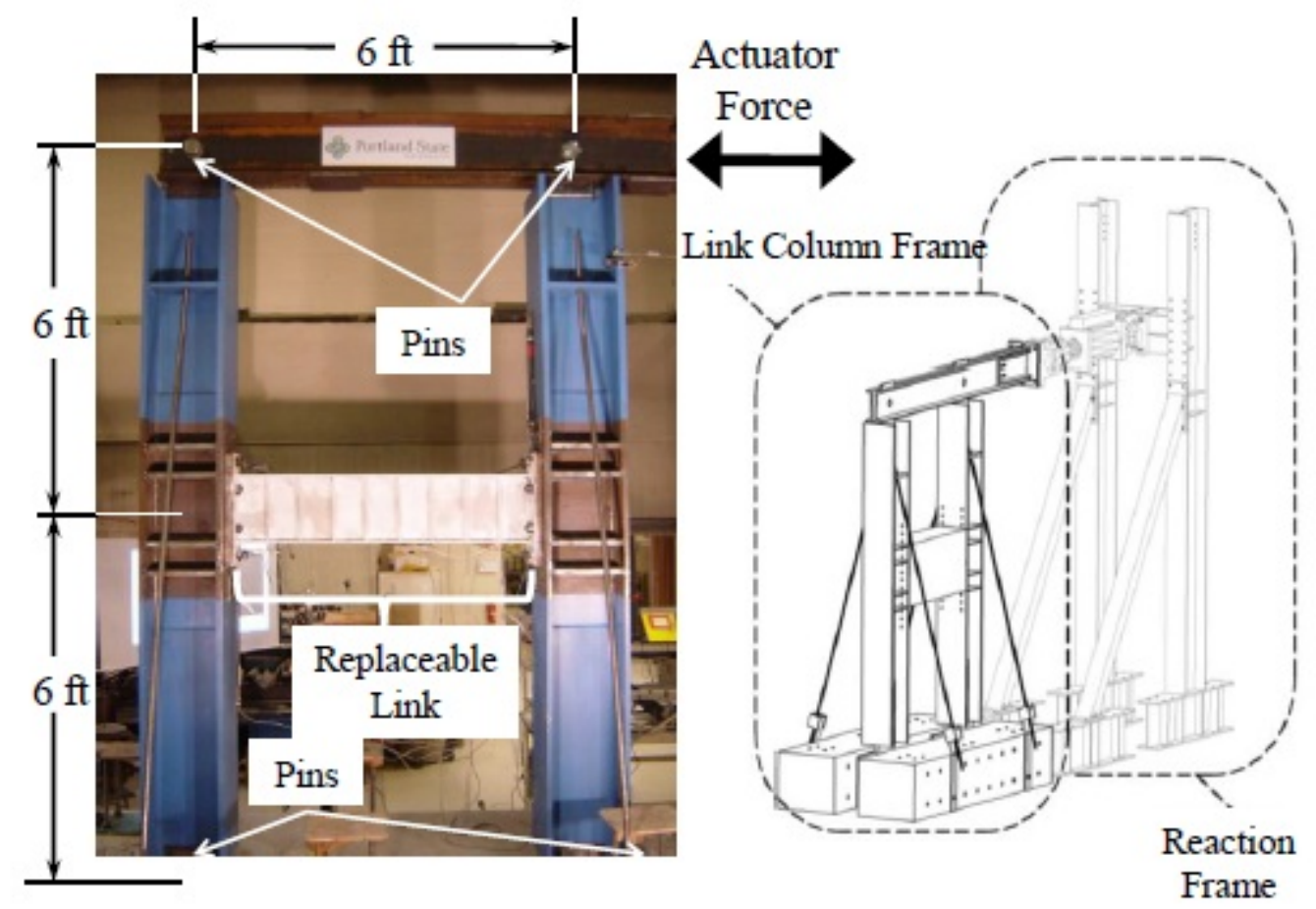

Figure 2.7: Experimental setup at iSTAR laboratory.

Laboratory test setup was designed based on the LCF concept, with two columns being tied through a link at mid-height as shown in Figure (2.7). The test setup was approximately $12 \mathrm{ft}$ tall, with column spacing at approximately $6 \mathrm{ft}$ on center, representing a full story frame. The link is the only aspect of the frame resisting the cyclic loading being applied by the 220 kip actuator at the top of the frame. 
Reducing the plastic demand at the location of welds was achieved through end stiffeners that shifted the plastic strain away from the welded link ends. Straight end stiffeners parallel to the web were found to be both practical and effective in reducing plastic strains at the link-to-end plate connection. These end stiffeners parallel to the web were also used in the full-scale LCF system experiments at nees@berkeley.

Stephens (2011) focused on the potential of shifting from discrete transverse web stiffening to continuously stiffened webs in built-up shear links within the LCF system. Built-up links were designed to yield in shear when subjected to severe cyclic loading and link webs were designed using two metal sheets joined by an elastic core. These composite steel-rubber-steel sandwich webs allowed for an increase in web thickness without increasing the shear strength of the links. Numerical and experimental investigations were conducted to assess the performance of composite sandwich links subjected to severe loading. Figure (2.8) shows the strain distribution of one of the links tested.

\subsubsection{Link Overstrength}

The maximum shear strength $\left(V_{\max }\right)$ that develops in the link can be different to the plastic shear $\left(V_{P}\right)$ capacity. The ratio of the maximum shear strength to the plastic shear capacity given by Equation (2.1) is referred to as overstrength and considers all factors that can contribute to the increased resistance (Dusicka et. al., 2006), including material strain hardening and the development of shear 

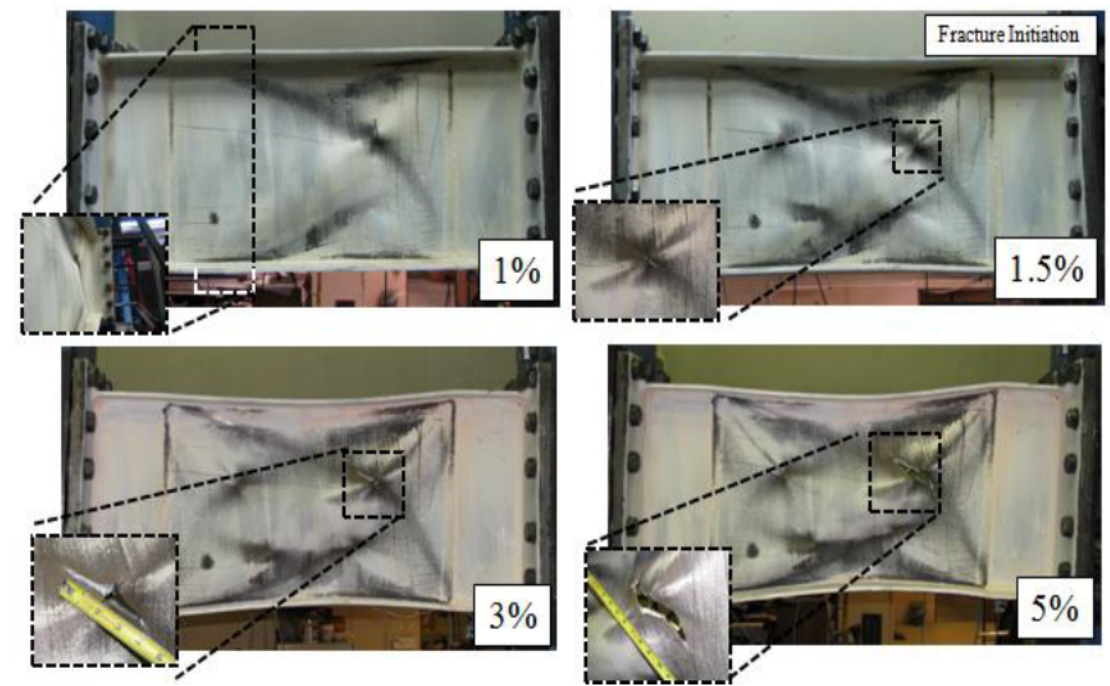

Figure 2.8: Strain distribution of link 25DBase.

resistance in the flanges.

$$
\Omega=\frac{V_{\max }}{V_{P}}
$$

Popov \& Engelhardt (1988) recommended a link overstrength factor of 1.5. Currently, the AISC Seismic Provisions (ANSI/AISC 341, 2010) specify a link overstrength factor of 1.25 for I-shaped links and 1.4 for box links for the design of diagonal braces and their connections, beams outside links and for the columns.

Okazaki et. al. (2005) evaluated link overstrength, particularly for sections with large ratios of flange to web area. This was based on a concern that heavy flanges can contribute substantially to the shear capacity of the section, and therefore generate high levels of overstrength. A range of link lengths were tested, ranging from short shear yielding links to very long flexural yielding 
links. The average overstrength for shorter link specimens was 1.41 and the average overstrength for longer link specimens was 1.22.

Tests on large built-up shear links for use in bridge applications showed overstrength factors of nearly 2.0 (McDaniel et. al., 2003). These tests differed from the others in that the specimens were built-up links with a larger section and different cross-section proportions than rolled W-shapes typically used for links in EBFs. Further, these links were also very short. This has led to concerns that current overstrength factors may be unconservative. Underestimating the overstrength developed in a shear link can lead to deficient designs and undesirable failure modes. Therefore, during the design process of the LCF system an overstrength of 2.0 was considered in order to estimate axial loads on foundations.

\subsection{Special Truss Moment Frames}

Special Truss Moment Frames (STMFs) dissipate energy via ductile segments located near the mid-span of truss girders and are suitable for high seismic areas. According to Chao \& Goel (2008) one advantage of using STMF systems is that the truss girders can be used over longer spans, and greater overall structural stiffness can be achieved by using deeper girders. On the other hand, the special segment is confined to within half of the span and the chords are continuous

over the ends of the special segment, resulting in similar difficulties for rapid return to occupancy as MRFs. Figure (2.9) illustrates a typical STMF. 


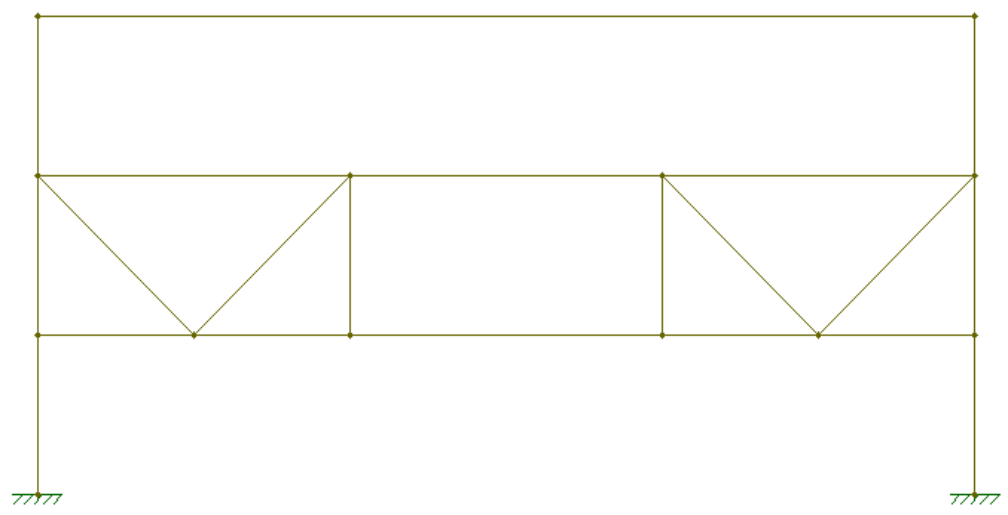

Figure 2.9: STMF with a single panel Vierendel ductile segment.

\subsection{Other Lateral-Load Resisting Frames}

The systems previously described are well suited to provide the desired level of ductility and energy dissipation under seismic loading for life safety without the need to design for elastic behavior. The resulting damage however can be induced for earthquake intensities well below the design level earthquakes because of the large response modification factor values used for sizing members; ranging from $\mathrm{R}=6$ to 8 (ASCE/SEI 7, 2010). The loss of occupancy and the difficulty associated with economically repairing the gravity system following an earthquake can burden the owners and occupants.

More recent lateral load-resisting systems introduced in the latest seismic design provisions for steel buildings (ANSI/AISC 341, 2010) offer additional options and potential advantages as compared to the conventional approaches. These systems include special steel plate shear wall and buckling-restrained 
braced frame. Both systems avoid using the primary gravity members for providing lateral inelasticity, but still exhibit some disadvantages when considering the potential for returning to occupancy.

Special Steel Plate Shear Walls (SPSWs) consist of a steel frame with steel infill web plates and the structural system has both horizontal and vertical boundary elements. Astanesh \& Zhao (2002) performed tests on 3-story SPSWs specimen to investigate the cyclic behavior of a steel plate shear wall system. One of the failure modes was the local buckling of the wide flange column which is a disadvantage when considering the potential for returning to occupancy. Figure (2.10) shows a special steel plate shear wall system.

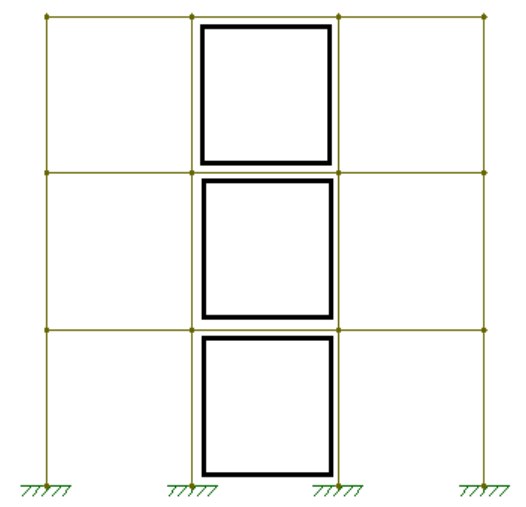

Figure 2.10: Special steel plate shear wall.

Buckling Restrained Braced Frames (BRBFs) use a prefabricated or manufactured brace element consisting of a steel core and a system of restraints that limits buckling of the steel core (ANSI/AISC 341, 2010). Experiments conducted by Roeder et. al. (2006) indicate that failure modes such as beam and column local buckling and fracture of the beam to gusset plate welds can occur 
outside of the brace. In addition, BRBFs have diagonal braces and the intent of the LCF research was to develop an alternative non-braced frame system. Figure (2.11) illustrates a buckling restrained braced frame.

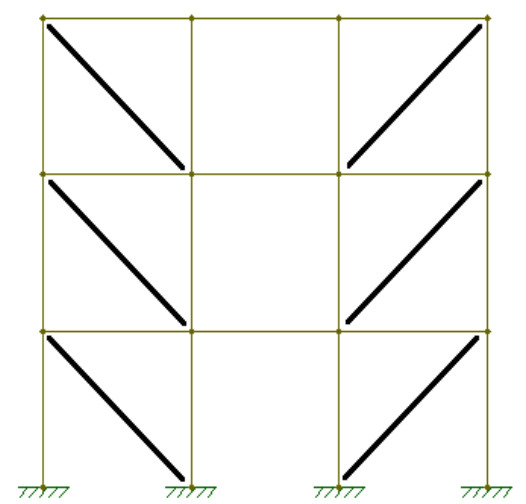

Figure 2.11: Buckling retrained braced frame.

Both of these systems are approaching the vision targeted by this research except that both utilize bays that are filled or have a diagonal brace. Other, not yet codified structural systems have been investigated including a moment resisting frame where nonlinear behavior is achieved through rocking in the form of post-tensioned moment connection rather than relying on material yielding (Ricles et. al., 2001). Although the system utilizes the gravity members for resisting lateral loads, the connections do not undergo inelastic deformation and therefore immediate occupancy level can be rapidly achieved. This damage free beam-column moment frame system has tremendous potential for achieving immediate occupancy due to the theoretical ability to self-center. However, even for these systems energy dissipation continues to be an important consideration and must be externally introduced in order to control the lateral drift (Filiatrault 
et. al., 2003).

Others have proposed self-centering systems that rely on rocking at the base of framing systems (Pollino \& Bruneau, 2007) and Khoo et. al. (2011), however, these systems could present numerous nonstructural issues in buildings for accommodating different vertical displacement at beams ends. More recently, two innovative systems of seismic-resistant steel frames with dissipative fuses were developed within the European Research Programme FUSEIS. The first, named FUSEIS 1, resembles a shear wall, whereas in the second system, named FUSEIS 2, the devices were made by introducing a discontinuity into the composite beams of a moment-resisting frame (Dimakogianni et. al., 2013). The cyclic experimental program consisted of testing only the columns with fuses in a one-story building.

Additional design options for lateral load-resisting structural systems need to be developed to propel state-of-the-art structural performance toward performance based design. Inelasticity is extremely beneficial to control the dynamic response and should not necessarily imply crippling damage to the gravity system. This dissertation addresses this gap in seismic design at the system level by developing and experimentally validating a new lateral load resisting system that can achieve specific target performance levels including rapid return to occupancy following an earthquake. 


\subsection{Experimental Methods for Structures}

Three methodologies are commonly used for evaluating the performance of structural systems when subjected to earthquake loads: shake table tests, quasistatic tests, and hybrid simulation. Although more realistic results can be achieved through shake table tests, only reduced-scale structural models can be tested due to table constraints. Also, shake tables with multi-degree of freedom capabilities are expensive to build. LCF system experiments were planned to be conducted at the University of Nevada, Reno considering a 2-story and 1-bay frame to investigate the dynamic inelastic behavior of the system and validate the performance based design methodology using real time earthquake simulation. Figure (2.12) illustrates the preliminary LCF experimental test setup.

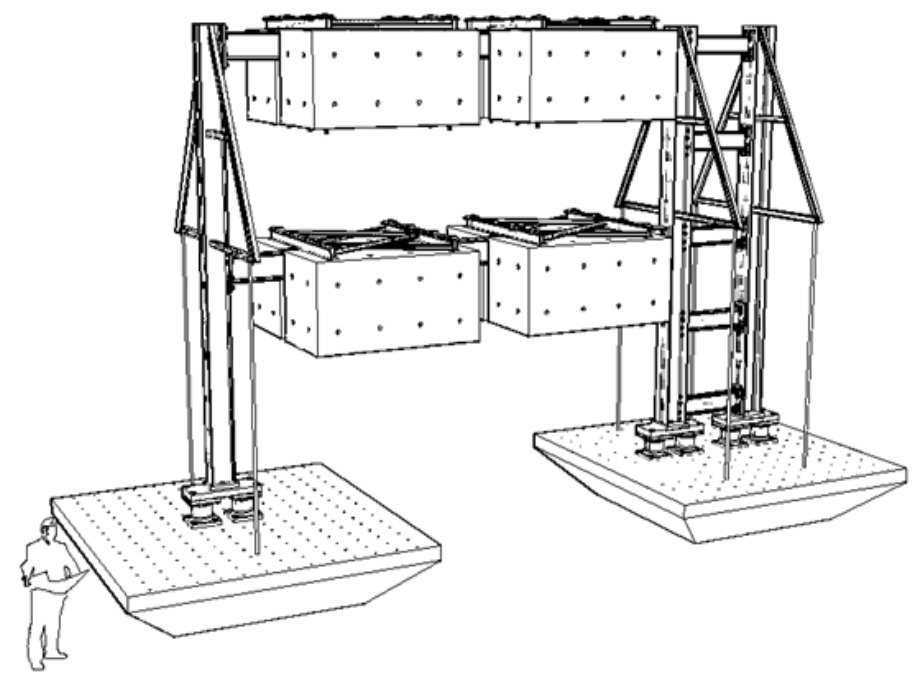

Figure 2.12: Preliminary LCF experimental test setup.

Quasi-static testing is a much simpler testing method that can be used to 
test structural members at large scales, but these tests require a predefined displacement history, that can later be difficult to relate to the seismic demands on the structure (Shing \& Mahin, 1984).

Hybrid simulation can be used to reduce the cost involved with fabrication and full-scale testing of large structures. Within a hybrid simulation test, critical componentes of the structural system under evaluation can be physically tested to be better understood, while others can be represented with computational models. The hybrid simulation test method is useful for modeling structures exhibiting complex non-linear behavior, especially if the non-linear behavior is concentrated in specific regions of the structure (Mosqueda et. al., 2005).

As mentioned in Chapter (1), the NEES site at the University of California at Berkeley (nees@berkeley) was utilized for the system level cyclic and hybrid simulation tests. The limitations of actuators as well as the overturning strong floor capacity were used as constraints to further refine the full-scale LCF experiments. 


\section{Chapter 3}

\section{Linked Column Steel Frame System}

\subsection{Introduction}

Non-braced frames are the lateral systems of choice when design constraints need to accommodate large openings. These structures are expected to achieve lateral ductility when subjected to earthquake loading through yielding beams or columns, and the connections must be capable of remaining intact through several cycles of inelastic rotation. The LCF system is a new lateral load resisting system that was developed at PSU with the goals of rapid return to occupancy via replacement of sacrificial components. The system consists of moment frames (MF) and linked columns (LC) with replaceable links. The MF provides gravity load-carrying capacity and under earthquake excitation the structure remains elastic. The LC consists of closely spaced dual-columns interconnected with links, which are designed to yield, deform plastically and be replaceable. The LCF system's ability to achieve rapid return to occupancy 
relies on the behavior of the replaceable links. The LCF system also offers architectural advantages of open perimeter bays and occupation versatility in the interior floor layout. Example LCF layout for a 3-story building is shown in elevation in Figure (3.1), where two LCs were utilized.

During the initial development of the system, non-moment transferring connections were introduced at all column to foundation locations and in strategic beam to column locations (Dusicka \& Iwai, 2007). These idealized pin connections at the base of each column limit yielding at the foundation and thereby minimize damage to the columns that is typical in ductile moment frame designs. The idealized pin connections in the MF beams reduce the lateral stiffness of the gravity moment frame. By superimposing the lateral response contributions of the LC and MF as shown in Figure (3.2), the resulting lateral response of the LCF system provides for three performance levels as follows: (i) Elastic behavior: Under service loads, the entire structure remains elastic and the primary stiffness is provided by the LC assembly. (ii) Rapid return to occupancy: Under extreme lateral loads, the links plastically deform while the rest of the structure remains elastic. (iii) Collapse prevention: MF beams are also damaged. The effectiveness of the rapid return to occupancy performance level depends on the relative transitions from elastic to plastic response of the LC and of the MF. Relative deformation can be described by the ratio of displacements at first yield $\Delta_{y L C} / \Delta_{y M F}$ from Equation (3.1), where $V_{L C}$ and $V_{M F}$ are the idealized lateral strengths, and $K_{L C}$ and $K_{M F}$ are the lateral stiffness values 
of LC and MF subcomponents, respectively.

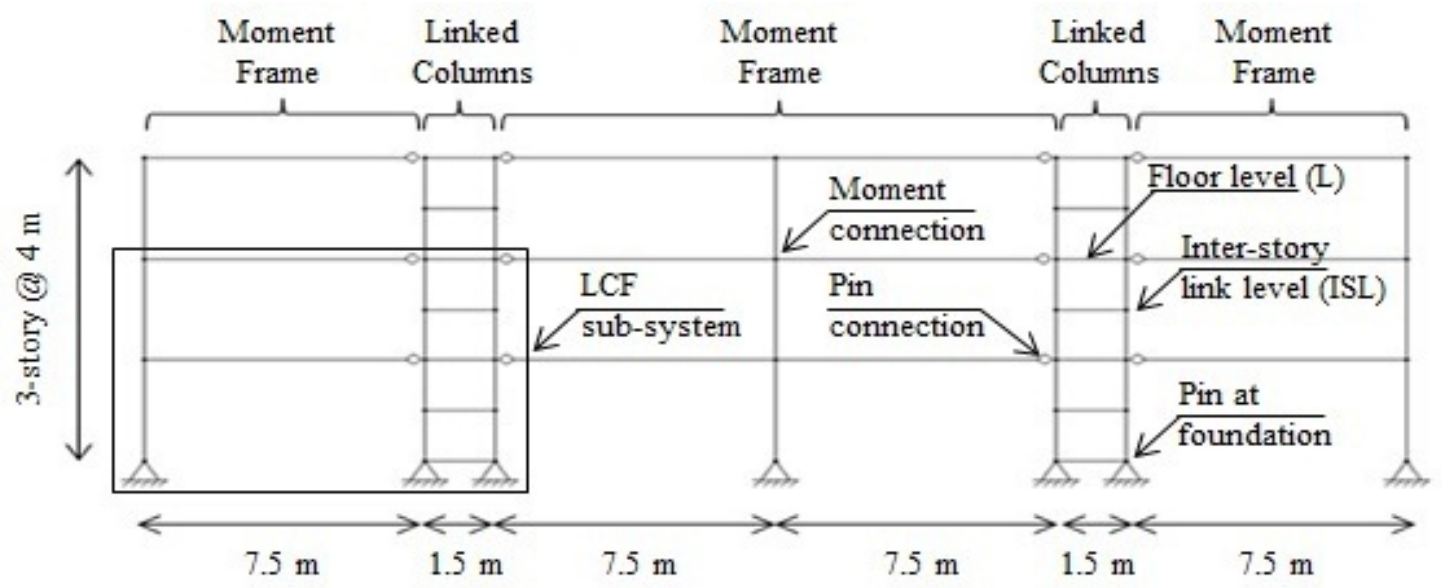

Figure 3.1: Elevation of a 3-story LCF building.

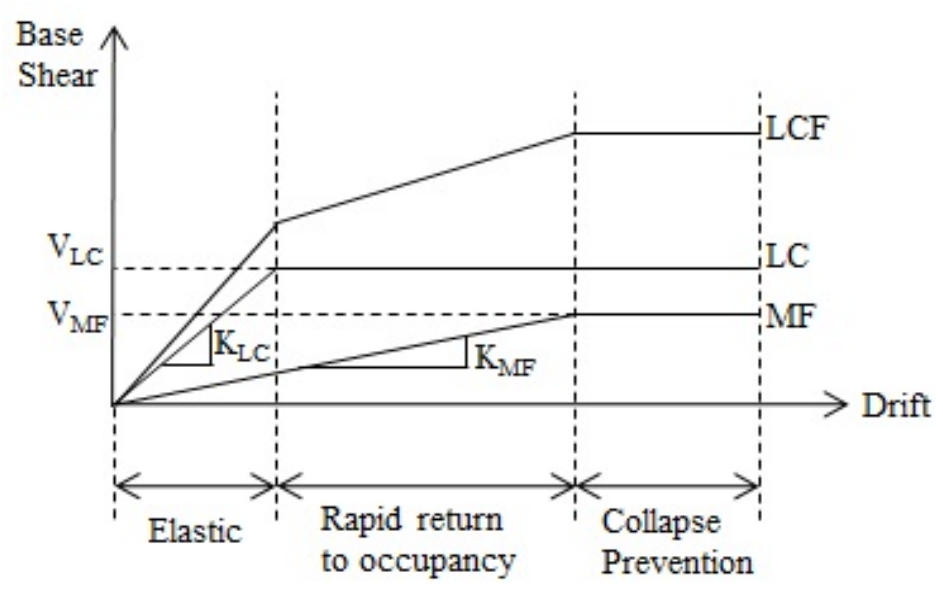

Figure 3.2: Idealized lateral response.

$$
\frac{\Delta_{y L C}}{\Delta_{y M F}}=\frac{V_{L C} K_{M F}}{V_{M F} K_{L C}}=\Gamma
$$

Ratios less than unity describe structural systems in which LC reaches plasticity at lower displacements than MF, thereby providing the potential for rapid return 
to occupancy performance level. The larger the difference between the yield displacements, the larger the drift range in which rapid return to occupancy can occur. Numerical and experimental studies has been carried out on bolted links that were either shear or moment dominated (Dusicka \& Lewis, 2010). The results have shown the viability of the links by their ability to deform plastically and exhibit failure modes that form away from the bolted connection regions. Other experiments on a steel frame similar to a linked column but with multiple sacrificial metal rods at mid-length of the links that were distributed throughout a story height had also indicated favorable cyclic response (Palkopoulou et. al., 2009). Also, system level numerical model development and analyses of the LCF system have been done considering time history analyses (Malakoutian, 2012) and non-linear static analyses (Lopes et. al., 2012 ${ }^{a}$ ) leading to preliminary design procedures for the LCF system toward performance level response.

\subsection{Prototype Buildings}

The seismic assessment of multistory special moment-resisting frames (SMRF) by the SAC Joint Venture (FEMA 355-C, 2000) after the 1994 Northridge earthquake revealed that many buildings using this type of lateral force resisting system did not perform as intended. The SAC model buildings were designed according to the 1994 Uniform Building Code and represented typical 3-, 9-, and 20-story office buildings in Los Angeles, Seattle and Boston. In elevation, the typical bay width was $9.1 \mathrm{~m}$ and typical bay height was $4.0 \mathrm{~m}$. 
For the purpose of this research, the location of the building is in Seattle, Washington. This location is considered a medium to high seismic design category. The resulting SMRF around the perimeter of the buildings developed by SAC included 3-story structures. Using the same design parameters, a 6story structure was also designed and beam and column sections for both LCF buildings are summarized in Table (3.1). These SMRF designs served as the benchmark for comparison with the LCF designs.

Table 3.1: Structural members for SMRF buildings.

\begin{tabular}{|c|c|c|}
\hline \multicolumn{3}{|c|}{ 3-story building } \\
\hline Story & Beam & Column \\
\hline 1 & W33x201 & $\mathrm{W} 14 \times 176$ \\
\hline 2 & W24x94 & $\mathrm{W} 14 \times 176$ \\
\hline 3 & W21x62 & W14x176 \\
\hline \multicolumn{3}{|c|}{ 6-story building } \\
\hline 1 & W36x231 & W14x730 \\
\hline $2-3$ & W33x141 & W14x730 \\
\hline $4-6$ & W27x114 & W14x665 \\
\hline
\end{tabular}

The LCF buildings followed similar layout, except that two of the columns were replaced by linked columns as shown in Figure (3.1). The LCFs were located around the perimeter of the building. Each linked column was spaced $1.5 \mathrm{~m}$ apart and the beam lengths were adjusted accordingly such that the building had the same overall plan dimensions. The links connecting the LCs were designed as shear links, also referred to as short links in EBFs. Link designs utilized built-up sections for additional design freedom and additional designs 
were also developed using wide flange beams. Unlike SMRFs, LCF systems have the potential repair in the rapid return to occupancy performance level because no gravity members would be damaged.

\subsection{Drift Sensitivity Analyses}

The initial member sizes of the LCFs were obtained using gravity load analyses along with accommodation for equivalent seismic lateral loads using building code prescribed forces (ASCE/SEI 7, 2010). Seismic design coefficients of $\mathrm{R}=8$ and $\mathrm{Cd}=5.5$ were used as per research done by Malakoutian (2012). Strong column weak beam capacity design principles were applied for all columns, including LCs. The initial LCF structure subjected to equivalent seismic lateral loads resulted in inter-story drifts exceeding code maxima. The determination of the controlling structural members in terms of drift within the structural system was accomplished through a series of parametric studies on a linear elastic numerical model subjected to the calculated equivalent lateral forces.

To investigate the stiffness sensitivity of individual members of the system, the flexural stiffness of each of the members was increased while all other members were kept unchanged. The flexural stiffness was altered numerically from 2 to 6 times the original in the model using a multiple of the moment of inertia for the member of interest. The gravity MF columns, MF beams, LC columns and the replaceable links were all independently considered. The impacts on inter-story drifts are summarized in Figure (3.3), where only the top three most 
significant parameters per story are reported for clarity.
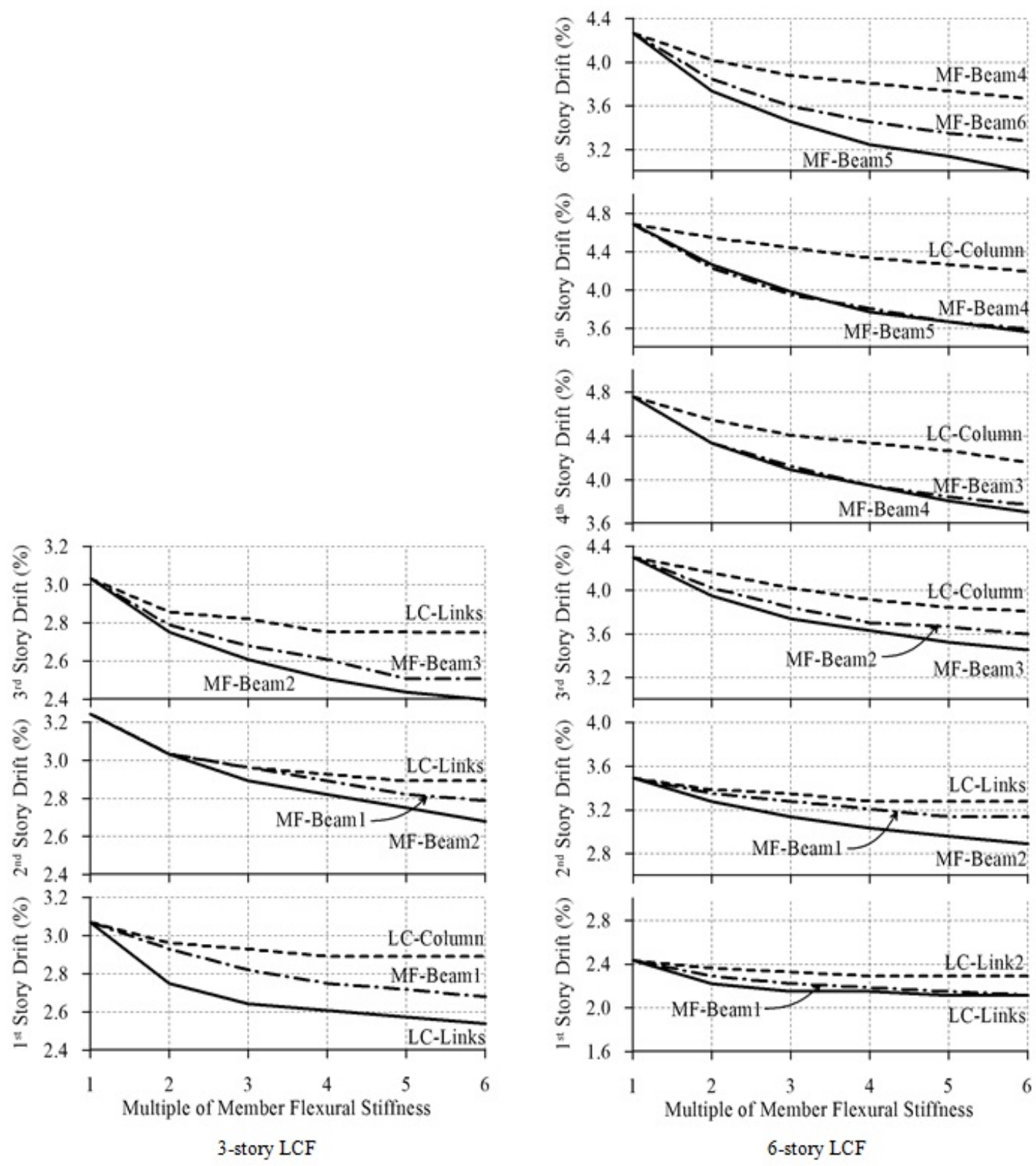

Figure 3.3: Inter-story drift sensitivity analyses.

Changing just one parameter did not necessarily address the desired drift criteria, however the results of the parametric study had identified key members that influenced the LCF system drift. For the three story building, the LC links 
significantly influenced the drift in the first story while MF beams exhibited more significant contributions in the upper stories. For the six story building, LC links as well as LC columns exhibited the most influence on the drift in the bottom stories with a diminishing role of the LC in the upper stories. The MF beams tended to be more influential at higher stories.

Two different design approaches were undertaken to determine the section sizes of LCF systems meeting the design intent of $2.5 \%$ inter-story drift limits; increasing MF beam sizes first and increasing LC links and column sizes first. Incremental increase in rolled wide flange sections available in the US was used for columns and MF beams. Built-up sections from plates as well as rolled wide flange alternatives were developed for the links resulting in LCF-L and LCF-M designs corresponding to the approach of controlling drift primarily using LC and MF, respectively. Member sizes were also checked for strong column weak beam such that the columns were expected to remain elastic. The resulting LCF 3 and 6 story systems are summarized in Table (3.2) for both approaches. Additional designs were completed using wide flange sections for shear links and were referred to as LCF-W design systems. 
Table 3.2: Structural members for LCF buildings

\begin{tabular}{|c|c|c|c|c|}
\hline \multicolumn{5}{|c|}{ 3-story LCF-L building } \\
\hline Story & $\mathrm{LC}$ & Link & Beam & GC \\
\hline 1 & $\mathrm{~W} 14 \times 257$ & A & $\mathrm{W} 24 \times 146$ & $\mathrm{~W} 14 \times 211$ \\
\hline 2 & $\mathrm{~W} 14 \times 257$ & $\mathrm{~A}$ & $\mathrm{~W} 24 \times 117$ & $\mathrm{~W} 14 \times 211$ \\
\hline 3 & $\mathrm{~W} 14 \times 257$ & B & $\mathrm{W} 21 \times 55$ & $\mathrm{~W} 14 \times 211$ \\
\hline \multicolumn{5}{|c|}{ 3-story LCF-M building } \\
\hline Story & $\mathrm{LC}$ & Link & Beam & GC \\
\hline 1 & $\mathrm{~W} 14 \times 233$ & A & $\mathrm{W} 24 \times 84$ & $\mathrm{~W} 14 \times 120$ \\
\hline 2 & $\mathrm{~W} 14 \times 233$ & $\mathrm{~A}$ & W24x68 & $\mathrm{W} 14 \times 120$ \\
\hline 3 & $\mathrm{~W} 14 \times 233$ & B & W21x55 & $\mathrm{W} 14 \times 120$ \\
\hline \multicolumn{5}{|c|}{ 6-story LCF-L building } \\
\hline Story & $\mathrm{LC}$ & Link & Beam & GC \\
\hline 1 & $\mathrm{~W} 14 \times 605$ & $\mathrm{C}$ & W30x99 & W14x398 \\
\hline 2 & W14x605 & $\mathrm{C}$ & W33x141 & W14x398 \\
\hline 3 & W14x605 & $\mathrm{C}$ & W36x182 & W14x398 \\
\hline 4 & W14x605 & $\mathrm{D}$ & W36x182 & W14x398 \\
\hline $5-6$ & $\mathrm{~W} 14 \times 550$ & $\mathrm{D}$ & W30x99 & W14x311 \\
\hline \multicolumn{5}{|c|}{ 6-story LCF-M building } \\
\hline Story & $\mathrm{LC}$ & Link & Beam & GC \\
\hline $1-2$ & $\mathrm{~W} 14 \times 730$ & $\mathrm{E}$ & W24x84 & W14x283 \\
\hline $3-4$ & $\mathrm{~W} 14 \times 730$ & $\mathrm{E}$ & W33x118 & W14x283 \\
\hline 5 & $\mathrm{~W} 14 \times 605$ & $\mathrm{D}$ & W33x118 & $\mathrm{W} 14 \times 283$ \\
\hline 6 & W14x605 & $\mathrm{D}$ & W24x84 & $\mathrm{W} 14 \times 283$ \\
\hline
\end{tabular}

All steel was assumed to be $345 \mathrm{MPa}$ nominal yield except for the links which were assumed to be $250 \mathrm{MPa}$. The LC links have been considered to be short or plastically shear dominated resulting in sectional properties summarized in Table (3.3). Intermediate or long links are also potentially possible in LCF 
designs, however the effects on meeting drift criteria and building member sizes had not been investigated in this research.

Table 3.3: LC links dimensions ( $\mathrm{mm}$ )

\begin{tabular}{ccccc}
\hline \multicolumn{5}{c}{ 3-story LCF-L building } \\
\hline Links & $h_{w}$ & $t_{w}$ & $b_{f}$ & $t_{f}$ \\
\hline $\mathrm{A}$ & 483 & 9.5 & 267 & 15.9 \\
$\mathrm{~B}$ & 356 & 9.5 & 267 & 15.9 \\
$\mathrm{C}$ & 762 & 12.7 & 267 & 15.9 \\
$\mathrm{D}$ & 546 & 9.5 & 267 & 15.9 \\
$\mathrm{E}$ & 864 & 12.7 & 432 & 25.4 \\
\hline
\end{tabular}

Structural steel weight was calculated for each of the frames as an indicator of cost of the structure and these are summarized in Table (3.4). The LCF systems in which the LC links and columns were used as the primary means of achieving drift criteria resulted in significantly lighter frames than those in which MF beams were used as means to control drift. These lighter LCF designs had comparable weights to SMRF designs indicating that comparable cost effectiveness is possible while considering the steel costs only. Hence, while the MF beam stiffness has greater influence on the drift than LC links or columns, more cost effective designs are achievable by altering the stiffness of LC links and columns as the starting point in controlling drift. 


\subsection{Lateral Performance}

\subsubsection{Numerical Model}

Non-linear lateral behavior of two dimensional frames was evaluated by incremental pushover analyses using SAP2000 (2010), which utilized lumped plastic hinge formulations based on FEMA 356 (2000). The numerical model included a $\mathrm{P}-\Delta$ column linked by axially rigid horizontal truss elements at each story. Pushover analyses were chosen for the ability to study and demonstrate the deformation mechanism, while assuming first mode dominated lateral response. Time history analyses can better represent the participation of higher mode effects, being covered elsewhere (Malakoutian, 2012).

\subsubsection{Lateral Response}

The system level pushover behavior of LCF and SMRF as measured by the roof drift and total frame base shear are shown in Figure (3.4). Both approaches used for design of members are included. Points where the first LC link and the first MF beam developed their respective plastic capacity are also indicated.

Each of the LCF systems exhibited three regions within the lateral response; elastic, yielding of LC link and yielding of LC links as well as MF beams. Provided the links are replaceable, these correspond to three distinct performance levels; elastic, rapid return to occupancy via replaceable links and collapse prevention. Unlike SMRFs, LCF systems have the potential for effective repair in 
the rapid return to occupancy performance level because no gravity members would be damaged. Unlike EBFs, the shear links are bolted to the columns to facilitate post-earthquake replacement. Even when residual drift results in the structure, the permanent deformations are locked in the shear links, which can be removed using a plasma cutter or an oxygen-acetylene torch. And, the elasticity of the MF component of the LCF system would provide restoring forces upon removal of the permanently deformed links.

The design base shear was included in Figure (3.4) to evaluate the efficiency of the designs. Since the SMRFs were drift controlled, an increase in beams size associated with addressing drift criteria also significantly increased the system strength and resulted in base shears that were at least $45 \%$ and $120 \%$ higher at first yield for the 3-story and 6-story LCF buildings, respectively. The LCF designs were found to have base shears closer to the design base shear both at first yield as well as at 5\% drift, a value that is twice the design drift limit. The lower base shears for the LCFs translates to lower demand on the foundation, which results in potential cost savings for the building. 


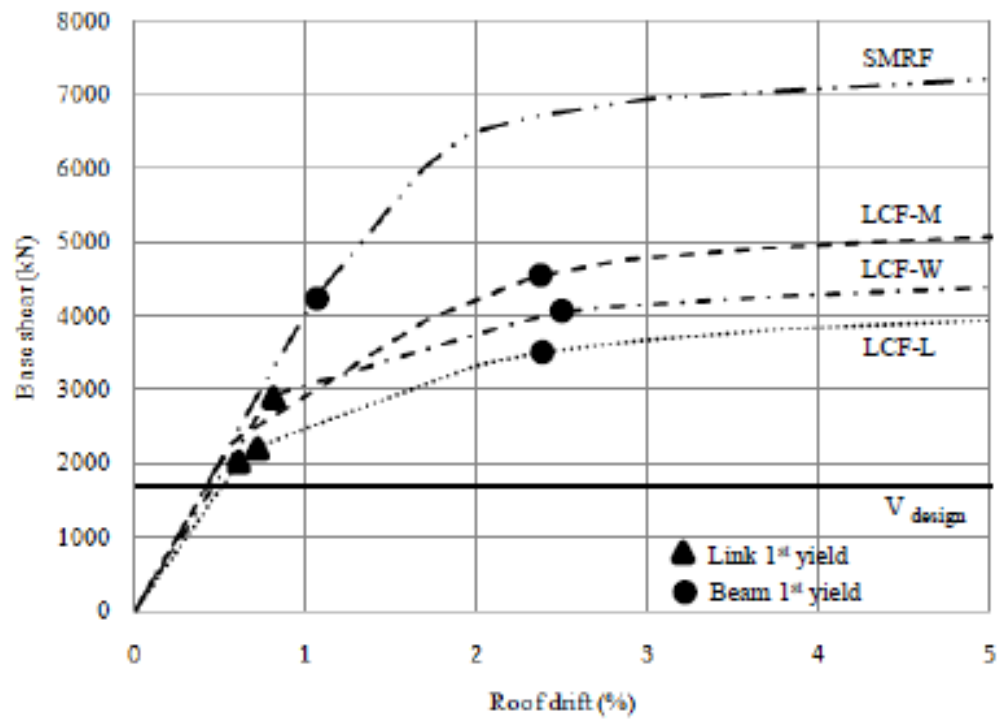

a) 3-story Buildings

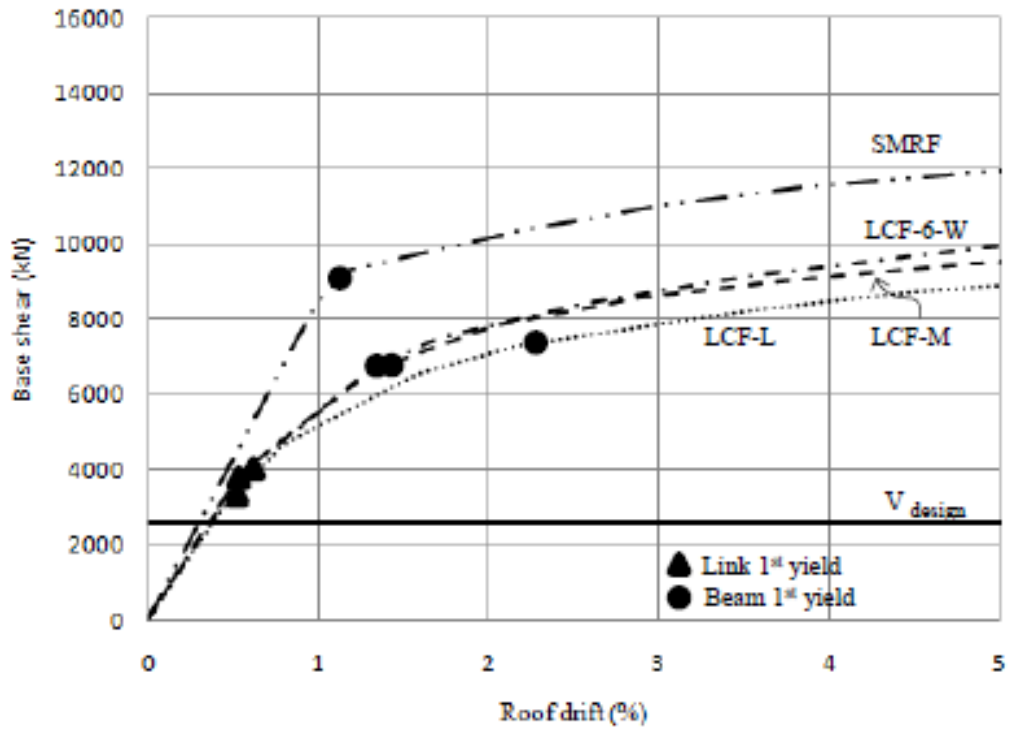

b) 6-story Buildings

Figure 3.4: Pushover comparisons.

\subsubsection{Performance Characteristics}

For the LCF systems, individual contributions from MF and LC subcomponents were determined with separate pushover analyses of a modified model where 
the member connections in MF and LC respectively were released, as shown in Figure (3.5). The building lateral response as measured by the roof drift was then expressed as a sum of the two subcomponents as illustrated in the idealized case of Figure (3.2). The difference to the idealized case was that system pushover results exhibited post yield stiffness and gradual transitions following first yield. Lateral system performance parameters were extracted to describe the overall system behavior.

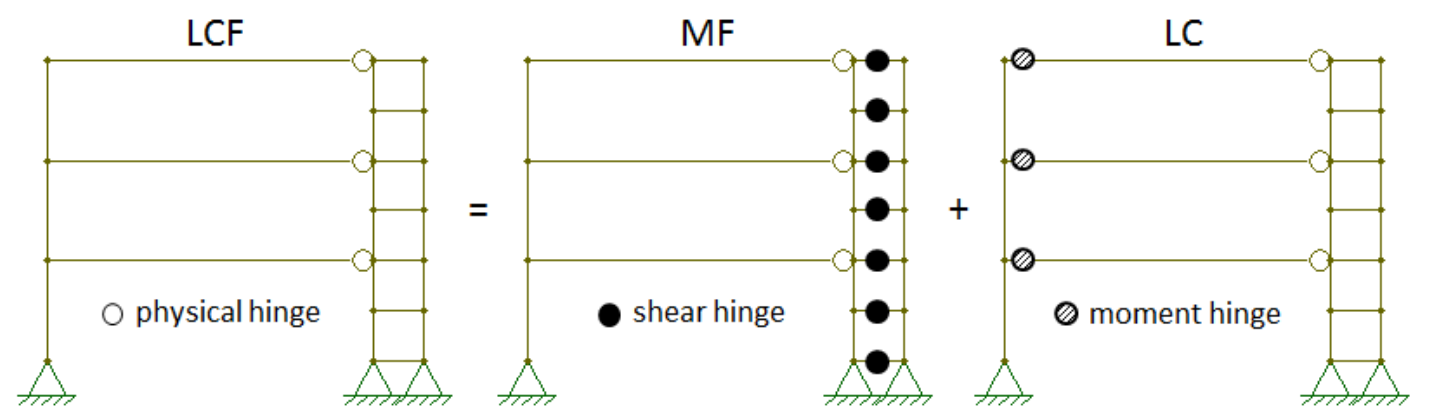

Figure 3.5: Modified model for a 3-story LCF building.

In addition to the drift ratio of Equation (3.1), stiffness and strength ratios $K_{L} C / K_{M} F=\alpha$ and $V_{L} C / V_{M} F=\beta$ were calculated. The lateral strength values were obtained at first yield, recognizing that additional strength is gained on subsequent plastic hinge formations and due to strain hardening. The system parameters are summarized in Table (3.4) and are useful in comparing the relative lateral performance of the overall systems.

For 3-story buildings the LCs dominated the stiffness as well as the strength of the overall structure while for 6-story buildings the LC strengths were below that of MF contributions. This difference explains in part the inter-story drift 
sensitivity of the 6 -story building to MF beam stiffness. In all but one LCF case, the displacement or drift ratio was equal to or less than 0.52 , indicating that rapid return to occupancy performance level, in which only LC links are damaged and all gravity members remain elastic, can occur over a drift range that is at least twice the drift of the first yield. The case in which the drift ratio was less favorable with regard to rapid return to occupancy was the 6story LCF-M design, where the MF beams were primarily used to achieve the prescribed drift criteria. In this case, the intended performance advantage of the LCF was less effective, suggesting that using LC links and columns first to meet drift is more advantageous.

Table 3.4: LCF system lateral parameters

\begin{tabular}{|c|c|c|c|c|}
\hline \multicolumn{5}{|c|}{ LCF 3-story building } \\
\hline & $\alpha$ & $\beta$ & $\Gamma$ & weight $\left(10^{3} \mathrm{~kg}\right)$ \\
\hline SMRF & - & - & - & 39.5 \\
\hline LCF-M & 2.91 & 0.86 & 0.30 & 50.3 \\
\hline LCF-L & 4.83 & 1.51 & 0.31 & 37.4 \\
\hline LCF-W & 5.36 & 1.78 & 0.33 & 38.9 \\
\hline \multicolumn{5}{|c|}{ LCF 6-story building } \\
\hline & $\alpha$ & $\beta$ & $\Gamma$ & weight $\left(10^{3} \mathrm{~kg}\right)$ \\
\hline SMRF & - & - & - & 178 \\
\hline LCF-M & 1.63 & 1.27 & 0.78 & 176 \\
\hline LCF-L & 3.08 & 1.27 & 0.41 & 170 \\
\hline LCF-W & 2.46 & 1.29 & 0.52 & 180 \\
\hline
\end{tabular}




\subsection{Preliminary Design Recommendations}

The LCF system is designed for strength and ductility. Strength is related to the maximum capacity of the structural member to resist a specific load and ductility is related to the maximum deformation beyond the yield stress without loss of strength. Based on sensitivity analyses Lopes et. al. $\left(2012^{a}\right)$ aimed at addressing drift limit considerations and their resulting lateral pushover response, suggesting the following steps for initial design of LCF buildings in medium to high seismic areas.

- Obtain and apply appropriate loads including equivalent static seismic loads as defined in ASCE/SEI 7 (2010).

- Proportion beams and columns as defined in AISC (2011).

- Check deflection limits of structural members as defined in IBC (2012).

- Proportion links as defined in ANSI/AISC 341 (2010) for EBFs. The links connecting the LCs are designed as shear links, also referred to as short links.

- Apply strong column and weak beam design and adjust member sizes accordingly as defined in ANSI/AISC 341 (2010). To prevent the failure during an earthquake the column member is designed stronger than the beam member using the strong column and weak beam concept. 
- Check inter-story drift as defined in ASCE/SEI 7 (2010). Should drift criteria not be met, increase the stiffness of the links and linked columns first before increasing moment frame beams.

- Check the relative LC and MF displacement using Equation (3.1) via non-linear pushover analyses as defined in FEMA 440 (2005).

- Refine the system strength by using links as built-up sections/wide flanges such that flexural stiffness of the links is minimally affected while decreasing the strength to reduce foundation forces.

Thus far the design recommendations rely on the designers ability to conduct pushover analyses to verify the potential of the system to achieve rapid return to occupancy. This may be viable for buildings where performance design is explicit, however, closed-form equations were developed to simplify the design procedures for routine structures while maintaining the outlined target performance levels. Those equations are discussed in Chapter (7). 


\section{Chapter 4}

\section{Numerical Analyses Toward Experimental Validation}

\subsection{Introduction}

This chapter outlines the LCF system analyses conducted in preparation for large-scale system experiments. Changing boundary conditions and using builtup sections for the shear links have shown an increase of LCF system's efficiency. Non-linear pushover analyses were used to validate the system ability to achieve the rapid return performance level. The objectives of these analyses were to design the physical specimens while considering laboratory constraints and as such have shed light on some of the important design considerations of the LCF system. In addition to the design of the physical specimens, a set of earthquakes was chosen in order to proceed with the hybrid simulation experiments; a combination of physical test of a critical experimental substructure tied to a numerical substructure component of the building. 


\subsection{Building Geometry}

The design resistance of the LCF was based on a prototype building that is a modified version of the 3-story building SAC configuration. The modification was due to physical space laboratory constraints. The building is three stories tall and has a $49.5 \mathrm{~m} \times 33 \mathrm{~m}$ plan with typical floor. In elevation, the typical bay width is $7.5 \mathrm{~m}$ and typical story height is $4 \mathrm{~m}$ and each LC is spaced $1.5 \mathrm{~m}$ apart. The building is located in Seattle, Washington, considered to be a medium to high seismic design category, with site class D as defined in ASCE/SEI 7 (2010). Seismic design coefficients for SMFRs of $R=8$ and $C_{d}=5.5$ were assumed because specific LCF system coefficients have not yet been established at the time these numerical analyses were performed. The SAC 3-story building has been extensively studied in the past, and this building was selected as the study building in this research because it is well known in the structural engineering research community and has served as a benchmark building in the structural analysis of moment-resisting frames. Lopes et. al. $\left(2012^{a}\right)$ designed both SMRF and LCF systems considering 3 and 6-story buildings. Figure (4.1) shows the plan view of the LCF building considered in the numerical analyses. 


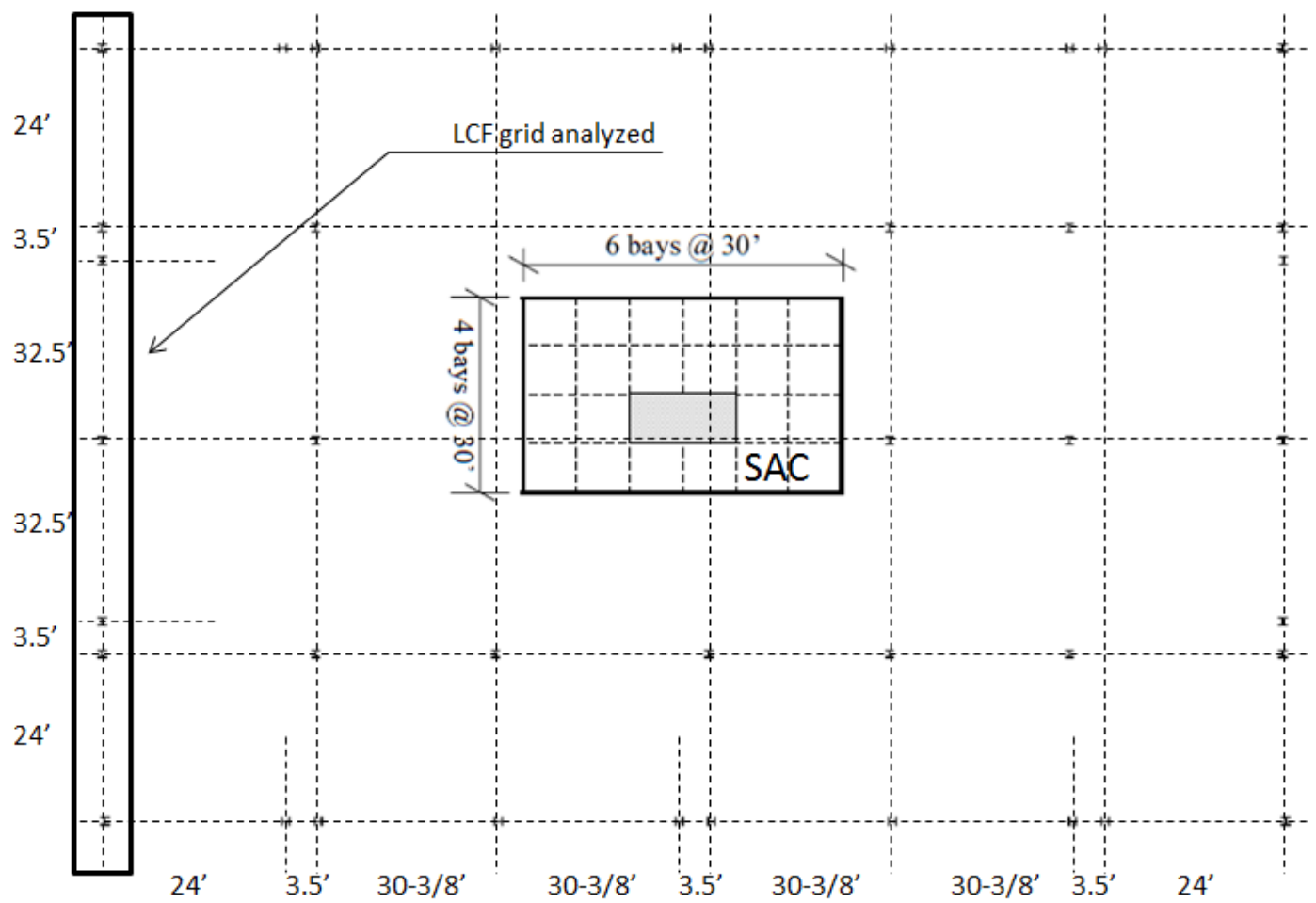

Figure 4.1: Plan view of the LCF building considered in the numerical analyses.

\subsection{System Efficiency}

The determination of the controlling members within the LCF system was accomplished through a series of parametric studies. Lopes et. al. (2011) started with a base model for the LCF system in which the inertial properties of frame members were increased incrementally by a succession of factors. Frame members were grouped according to function and story height. Each frame member group was individually altered by increasing the sectional properties for moment of inertia by a succession of factors from the integers and half-values from 1 to 6 . These parametric studies successfully identified the controlling frame members of the LCF structural system thereby affording the designer precise 
control over the system as a whole. These studies were repeated for 1,2, 3 and 6 story models of the LCF system leading to a preliminary LCF design (Lopes et. al., 2012 $2^{a}$.

The next step was to analyze the LCF buildings considering two aspects: 1) change in links' boundary conditions and 2) use of built-up sections as shear links. As such, four different numerical models were designed as per the preliminary LCF design procedure described in Chapter (3) to determine the section sizes of LCF systems meeting the design intent of $2.5 \%$ inter-story drift limits. Figure (4.2) shows different boundary conditions that were used for the links. Should be mentioned that all analyses consisted of a 3-story and 4-bay LCF building. LCF-B-ISL (a) is a system considering built-up sections for the links at inter-story levels of the structure, whereas LCF-B-L $(b)$ is a system considering built-up sections for the links at both floor and inter-story levels of the structure. LCF-W-ISL (a) is a system considering wide flanges for the links at inter-story levels of the structure, whereas LCF-W-L $(b)$ is a system considering wide flanges for the links at both floor and inter-story levels of the structure. All steel is assumed to be $345 \mathrm{MPa}$ nominal yield stress except for the links which are assumed to be $250 \mathrm{MPa}$. The results for LCF 3 story systems are summarized in Table (7.4). The LC links have been considered to be short and plastically shear dominates resulting in section properties summarized in Table (4.2).

Lateral behavior of the linked column frame is evaluated by incremental 


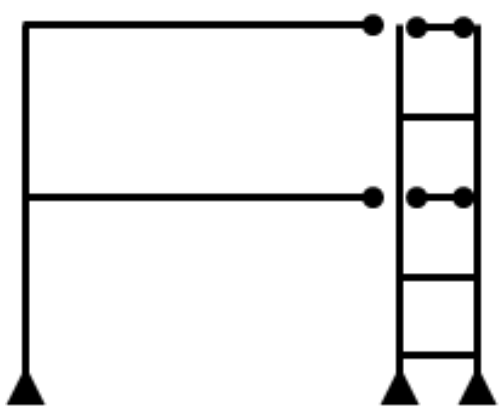

(a)

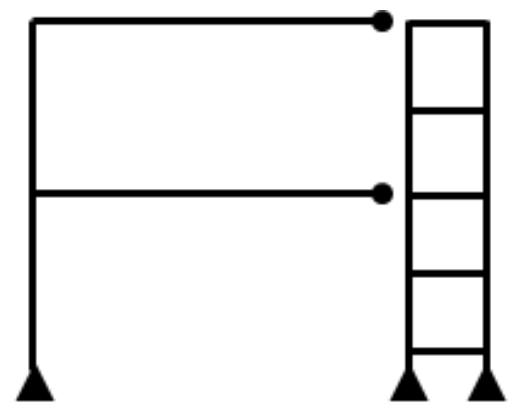

(b)

Figure 4.2: Different boundary conditions for links.

Table 4.1: Structural members for LCF buildings.

\begin{tabular}{|c|c|c|c|c|c|}
\hline \multirow[t]{2}{*}{ Type } & \multirow[t]{2}{*}{ Story } & \multicolumn{2}{|c|}{ LC } & \multicolumn{2}{|c|}{ MF } \\
\hline & & Column & Link & Beam & Column \\
\hline \multirow[t]{3}{*}{ LCF-B-ISL } & 1 & W14x132 & A & W24x62 & W14x120 \\
\hline & 2 & W14x132 & A & W24x62 & $\mathrm{W} 14 \times 120$ \\
\hline & 3 & W14x132 & A & W18x50 & $\mathrm{W} 14 \times 120$ \\
\hline \multirow[t]{3}{*}{ LCF-W-ISL } & 1 & W14x132 & W12x96 & W24x62 & W14x120 \\
\hline & 2 & W14x132 & W12x96 & W24x62 & W14x120 \\
\hline & 3 & W14x132 & W12x96 & $\mathrm{W} 18 \times 50$ & $\mathrm{~W} 14 \times 120$ \\
\hline \multirow[t]{3}{*}{ LCF-B-L } & 1 & W14x132 & B & W24x62 & W14x120 \\
\hline & 2 & W14x132 & B & W24x62 & $\mathrm{W} 14 \times 120$ \\
\hline & 3 & W14x132 & B & W18x50 & W14x120 \\
\hline \multirow[t]{3}{*}{ LCF-W-L } & 1 & W14x132 & W12x50 & W24x62 & $\mathrm{W} 14 \times 120$ \\
\hline & 2 & W14x132 & W12x50 & W24x62 & $\mathrm{W} 14 \times 120$ \\
\hline & 3 & W14x132 & $\mathrm{W} 12 \times 50$ & W18x50 & $\mathrm{W} 14 \times 120$ \\
\hline
\end{tabular}

pushover analysis using SAP2000 (2010), which utilized lumped plastic hinge formulations based on FEMA 356 (2000). In this method inelastic material behavior with p-delta effects were included. The plastic hinges at beams and columns were modeled to have elasto-plastic behavior considering the effect of the interaction of moment and axial load. The system level pushover behavior of LCF systems measured by overall base shear versus roof drift is shown in Figure (4.3). LCF systems considering wide flanges as shear links indicated a larger overall base shear. LCF-B-L and LCF-B-ISL systems indicated a preference to be experimentally tested. The lower base shears for the LCFs translates to 
Table 4.2: Structural members for LCF buildings.

\begin{tabular}{ccccccc}
\hline Links & $\mathrm{hw}(\mathrm{mm})$ & $\mathrm{tw}(\mathrm{mm})$ & $\mathrm{bf}(\mathrm{mm})$ & $\mathrm{tf}(\mathrm{mm})$ & $\mathrm{Vp}(\mathrm{kN})$ & $\mathrm{Mp}(\mathrm{kN} . \mathrm{m})$ \\
\hline $\mathrm{A}$ & 260 & 9.5 & 368 & 32 & 335 & 295 \\
$\mathrm{~B}$ & 152 & 9.5 & 368 & 32 & 195 & 170 \\
\hline
\end{tabular}

lower demand on the foundation, which results in potential cost savings for the building.

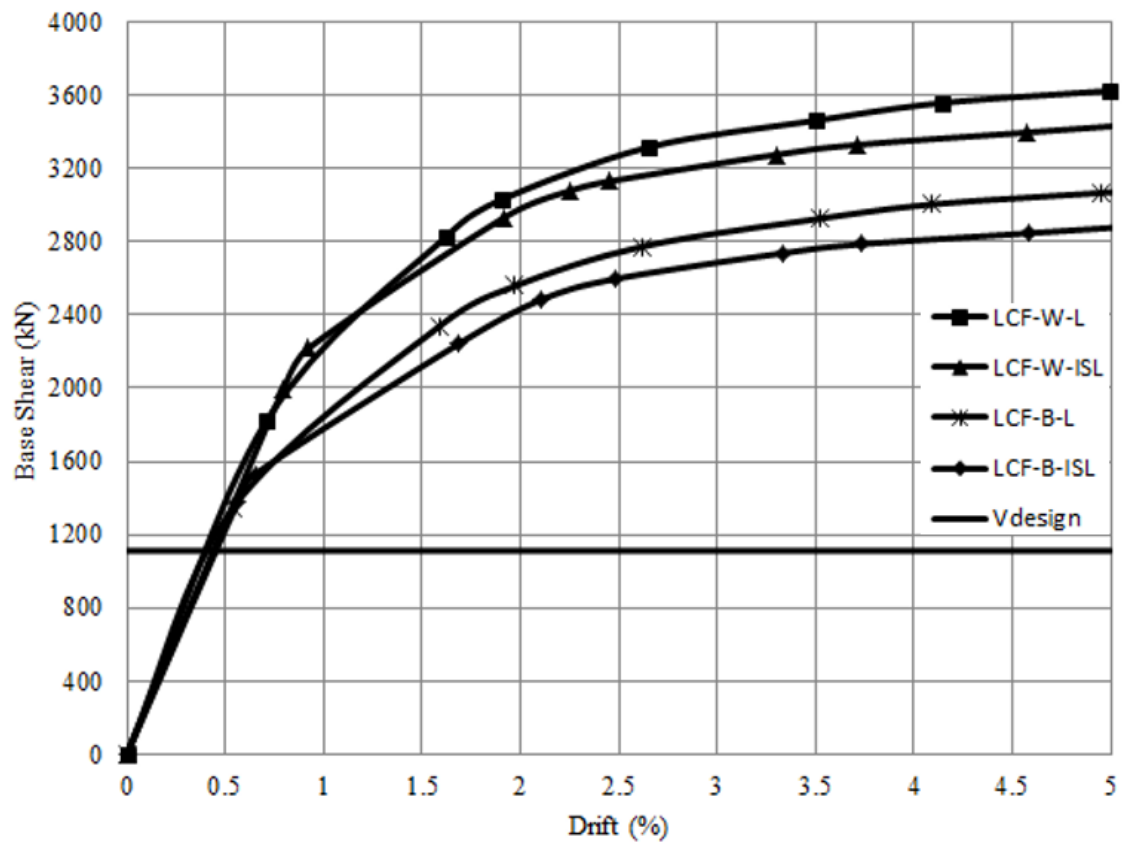

Figure 4.3: Pushover response for different LCF systems.

For the LCF systems shown in Figures (4.4) and (4.5), individual contributions from LC and MF subcomponents were determined with separate pushover analyses of a modified model where the member connections in MF and LC respectively were released. Points where the first LC link and the first MF beam developed their respective plastic capacity indicate the rapid return to occupancy performance level. 
The building lateral response as measured by the roof drift was then expressed as a sum of the two subcomponents as illustrated in the idealized case of Figure (3.2). The difference to the idealized case is that system pushover results exhibited post yield stiffness and gradual transitions following first yield. The LCF-B-ISL and LCF-B-L systems were found to have base shears closer to the design base shear both at first yield as well as at $5 \%$ drift, a value that is twice the design drift limit. Also, the LCF philosophy of exhibiting rapid return to occupancy performance level was improved considering built-up sections for the links instead of wide flanges. Each of the LCF systems exhibited three regions within the lateral response; elastic, yielding of LC link and yielding of LC links as well as MF beams.

Lateral system performance parameters were extracted to describe the overall system behavior. In addition to the drift ratio of Equation (3.1), stiffness and strength ratios $K_{L C} / K_{M F}$ and $V_{L C} / V_{M F}$ were calculated. The lateral strength values were obtained at first yield, recognizing that additional strength is gained on subsequent plastic hinge formations and due to strain hardening. The system parameters, the first LC link and the first MF beam plasticity drift are summarized in Table (4.3).

Table 4.3: LCF system lateral parameters.

\begin{tabular}{|c|c|c|c|c|c|c|}
\hline Type & $K_{L C} / K_{M F}$ & $V_{L C} / V_{M F}$ & $\Delta_{L C} / \Delta_{M F}$ & $1^{\text {st }}$ LC yield (\%) & $1^{\text {st }}$ MF yield (\%) & RR (\%) \\
\hline LCF-B-ISL & 2.65 & 0.81 & 0.30 & 0.56 & 2.1 & 1.54 \\
\hline LCF-W-ISL & 2.76 & 1.18 & 0.43 & 0.79 & 1.91 & 1.12 \\
\hline LCF-B-L & 2.87 & 0.91 & 0.32 & 0.53 & 1.97 & 1.44 \\
\hline LCF-W-L & 2.96 & 1.24 & 0.42 & 0.71 & 1.90 & 1.19 \\
\hline
\end{tabular}


For 3-story buildings the LCs dominated the stiffness as well as the strength of the overall structure. In all cases analyzed, the drift ratio $\Delta_{L C} / \Delta_{M F}$ is equal to or less than 0.43 , indicating that rapid return to occupancy performance level, in which only LC links are damaged and all gravity members remain elastic, can occur over a drift range that is at least twice the drift of the first yield. 

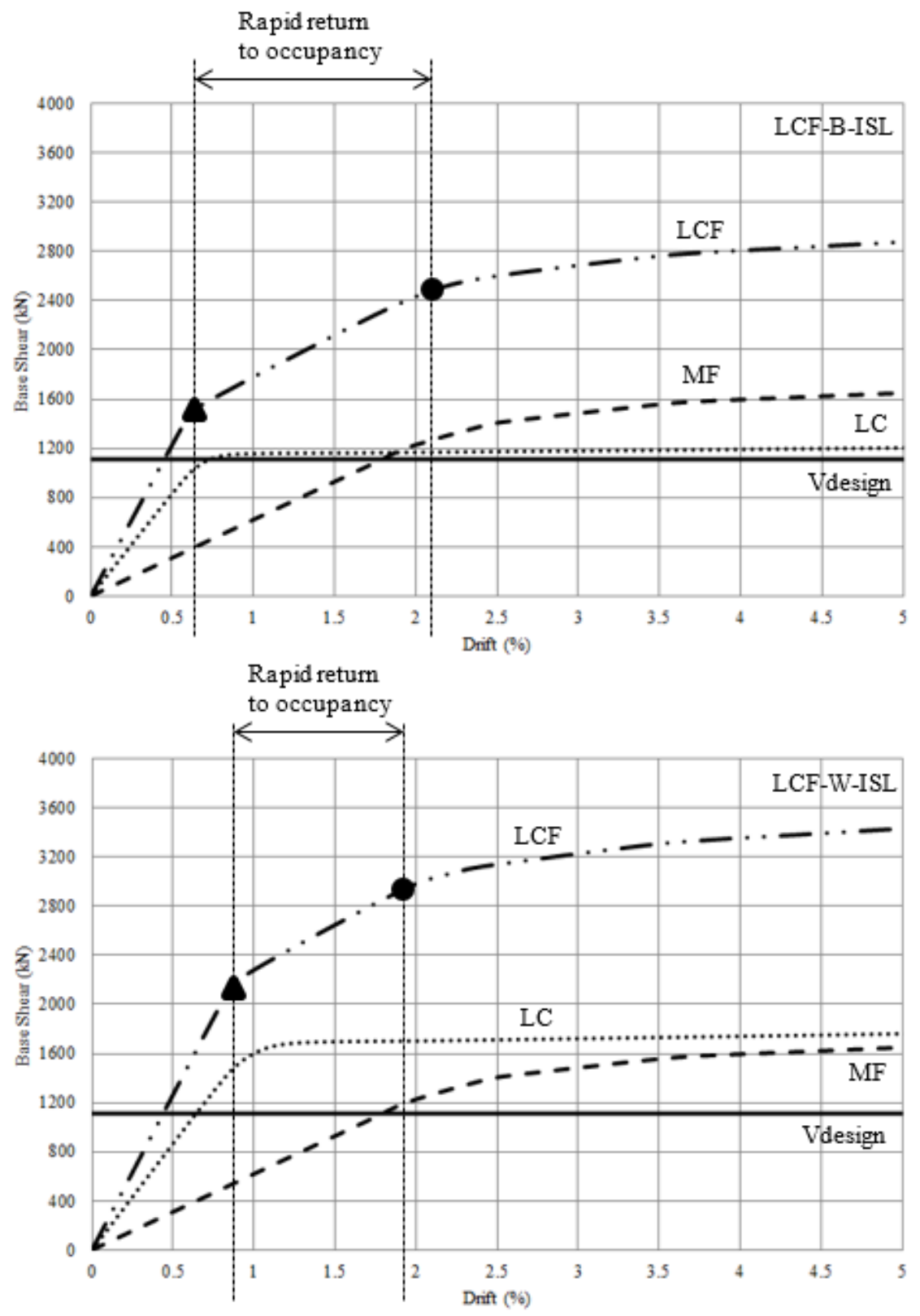

Figure 4.4: Element contribution to LCF-B-ISL and LCF-W-ISL systems. 

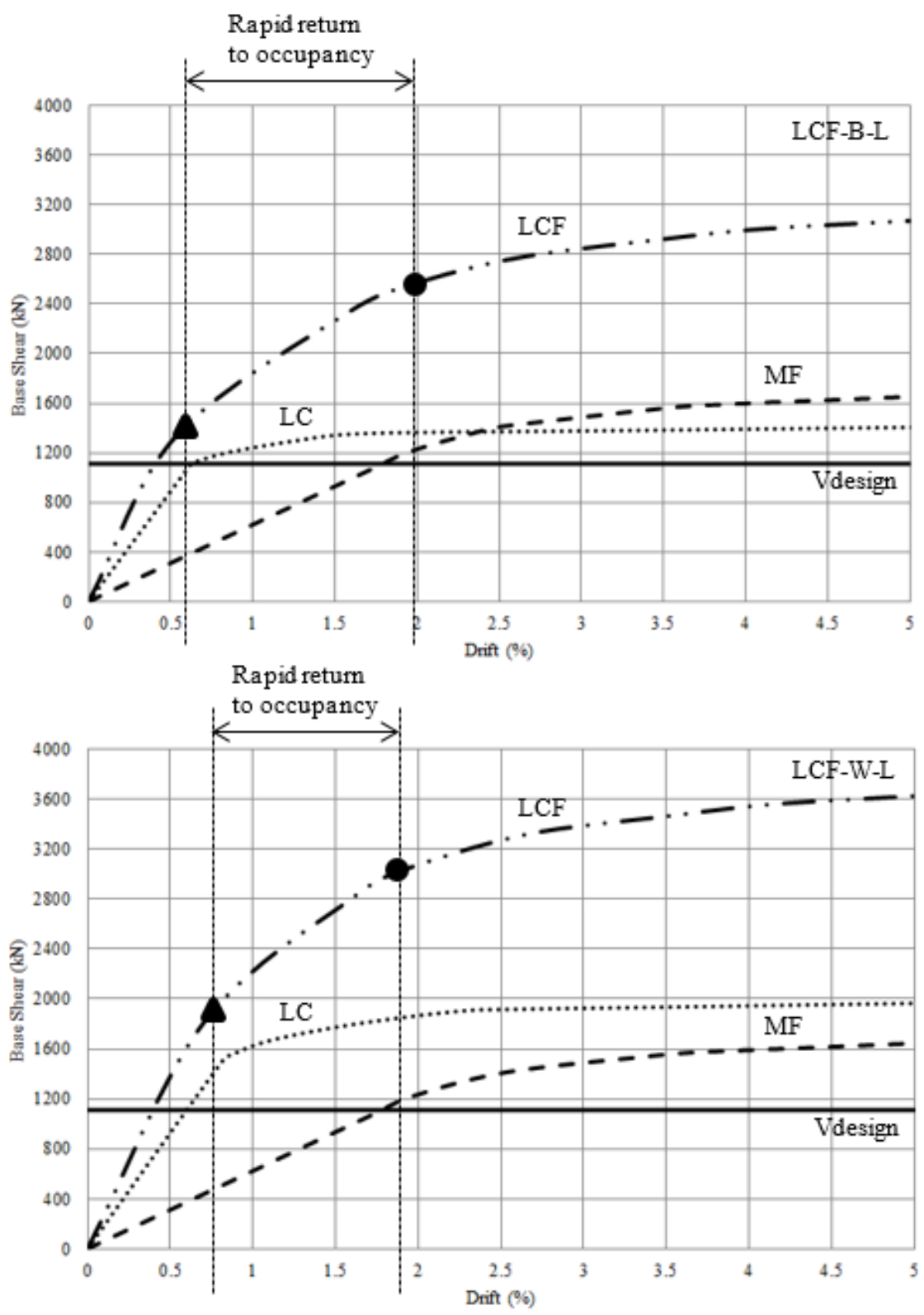

Figure 4.5: Element contribution to LCF-B-L and LCF-W-L systems.

\subsection{LCF Building Selection}

Even though 3-story LCF-B-L and LCF-B-ISL systems indicated a preference to be experimentally tested, a different experimental substructure was chosen. 
The reasons were as follows:

- Laboratory constraints revealed that the LCF system could be implemented up to a 2-story building.

- The LCF system required two actuators per floor and only four actuators were available.

- Using only one type of connection for the linked columns dictated the two specimens to be tested. For the first specimen with five links, denoted hereafter as LCF-5L, the wide flange links were placed throughout the height of the building, whereas for the second specimen with three links, denoted hereafter as LCF-3L, the wide flange links were placed at floor levels only.

- The research project had tight budget limits in terms of ordering built-up sections. Welding cost would have increased the budget.

Thus, the LCF building selected for the experimental test using hybrid simulation scheme consisted of a 2-story and 4-bay structure with replaceable links as wide flanges. The building plan was considered to be symmetric about the $x$ and $y$ axes, but the mass centers were shifted by $5 \%$ of plan dimension in both directions to account for accidental torsion, as required by (ASCE/SEI 7, 2010) for dynamic analysis. However, only one grid of the LCF system was analyzed as shown in Figure (4.1). Slab action was accounted for through a 
rigid diaphragm constraint, which allowed the mass and rotational inertia to be distributed to the entire floor.

Malakoutian (2012) performed nonlinear dynamic analyses of LCF buildings using OpenSees and his model was used as a reference. Therefore, twodimensional models were created in OpenSees to represent two different LCF buildings. The first building consisted of a 2-story and 4-bay LCF building and had five links per linked column, LCF-5L, and the second building consisted of a 2-story and 4-bay LCF building and had three links per linked column, LCF-3L. Figures (4.6) and (4.7) show an overview of the numerical model for specimens LCF-5L and LCF-3L, respectively, including rigid end offsets. While beam-to-column connections and link-to-column connections were assumed to be perfectly rigid, non-moment transferring connections were introduced at all column to foundation locations and in strategic beam to column locations.

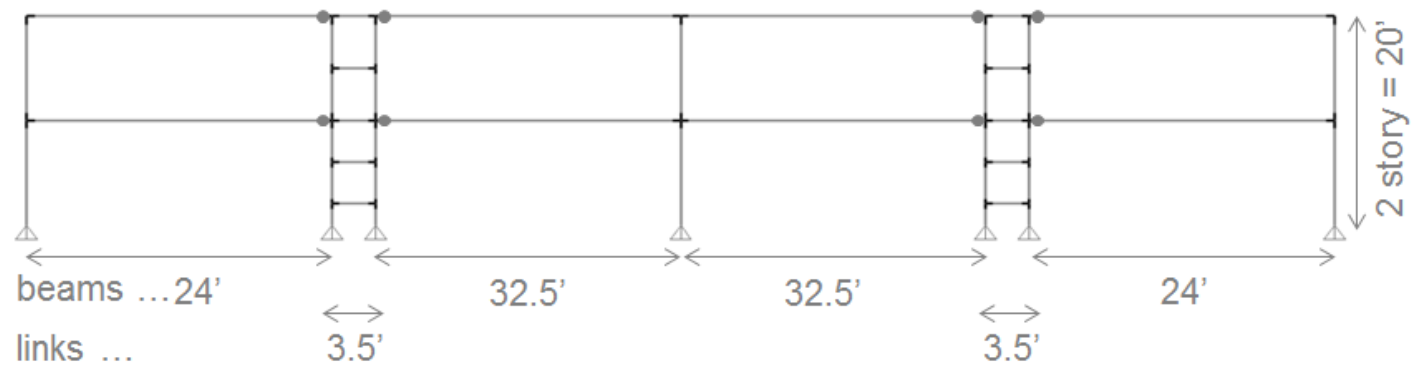

Figure 4.6: Numerical model for specimen LCF-5L.

The yield strength of structural steel $f_{y}$ was taken to be $50 \mathrm{ksi}$ and modulus of elasticity $E$ equals to 29,000 ksi. All beams and columns were modeled using force-based nonlinear beam-column elements that model the spread of plasticity 


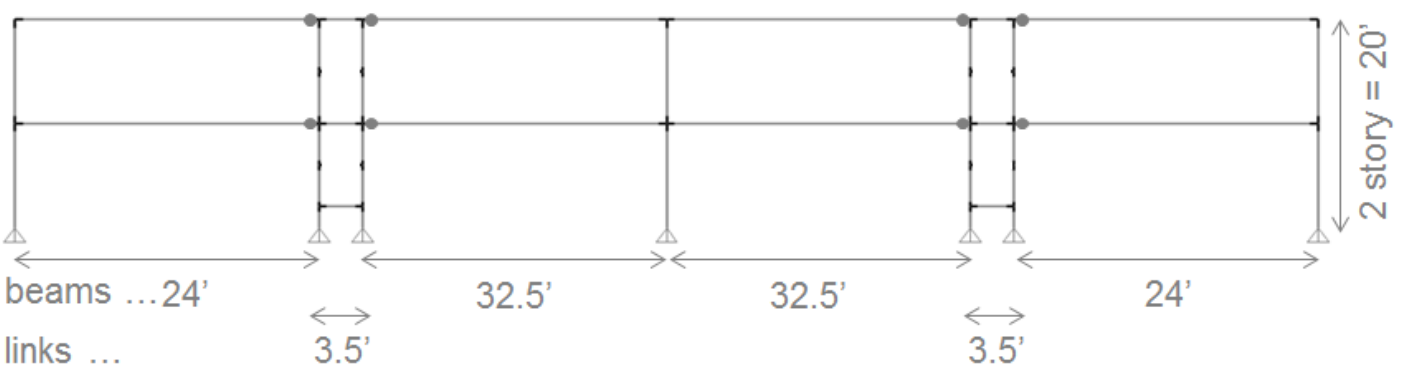

Figure 4.7: Numerical model for specimen LCF-3L.

across the element, with fiber sections that essentially account for momentaxial force interaction at each analysis step. All columns were pinned at the base and Rayleigh damping ratio of $2 \%$ at the first mode was used. Damping comparison studies of a 3-story conventional building performed by (Sayani et. al., 2011) indicated only a trivial difference in median responses between Rayleigh damping and stiffness proportional damping. Also, replaceable links were modeled based on experimental results from Dusicka \& Lewis (2010).

P-delta effects were also included in the analysis. Gravity loads acting on the deformed configuration of the structure amplify story drift especially under severe earthquake motions. Bernal (1987) proposed an empirical formula that characterizes gravity loads to account for P-delta effects in inelastic structures subjected to earthquakes. Based on the results, he concluded that the code previsions were inadequate and not conservative enough to account for P-delta effects. Gupta \& Krawinkler (2000) illustrated how the response of steel frames is very sensitive to p-delta effects and not simple to predict. According to Gupta \& Krawinkler (1999) the P-delta effects caused by the vertical loads tributary 
to the interior cannot be ignored, thus their structures were modeled through a leaning column.

Thus, the leaning column approach was used to take into account the Pdelta effects in the LCF system. The leaning column was pinned at the base and connected to the frame by axially rigid link elements and was modeled with elastic column elements. The model consisted of a very small moment of inertia in order to have negligible effect on the lateral stiffness of the structure and a very large area in order to create axially rigid elements.

\subsection{Earthquake Selection}

Ground motion intensity was selected such that three distinct performance levels were induced in the LCF specimen: linearly elastic (E), rapid return to occupancy (RR1 and RR2) corresponding to a moderate damage state, and collapse prevention (CP1 and $\mathrm{CP} 2)$ corresponding to a significant damage state. The ground motions were those developed in the SAC project for the Seattle site (Somerville et. al., 1997). The ground motion for the linearly elastic intensity was obtained taking $15 \%$ of SE-05. Table (4.4) below provides information on the records generated for Seattle having probabilities of exceedence of $10 \%$ in 50 years (SE-05 and SE-20) and 2\% in 50 years (SE-25 and SE-29). These acceleration time histories have been derived from historical recordings and have been altered so that their mean response spectrum matches the NEHRP (1997) design spectrum, modified from soil type of $S_{B}$ and $S_{C}$ to soil $S_{D}$ and having 
a hazard specified by the 1997 United States Geological Survey (USGS) maps. Response design spectra and acceleration time history are illustrated in Figure (4.8) and Figure (4.9), respectively.

Table 4.4: Details of Seattle ground motions.

\begin{tabular}{ccccc}
\hline Name & Code & Record & Magnitude & Scale Factor \\
\hline SE-05 & RR1 & Olympia, 1949 & 6.5 & 1.86 \\
SE-20 & RR2 & Vina del Mar, 1949 & 8.0 & 1.69 \\
SE-25 & CP1 & Olympia, 1949 & 6.5 & 4.35 \\
SE-29 & CP2 & Valparaiso, 1985 & 8.0 & 2.9 \\
\hline
\end{tabular}

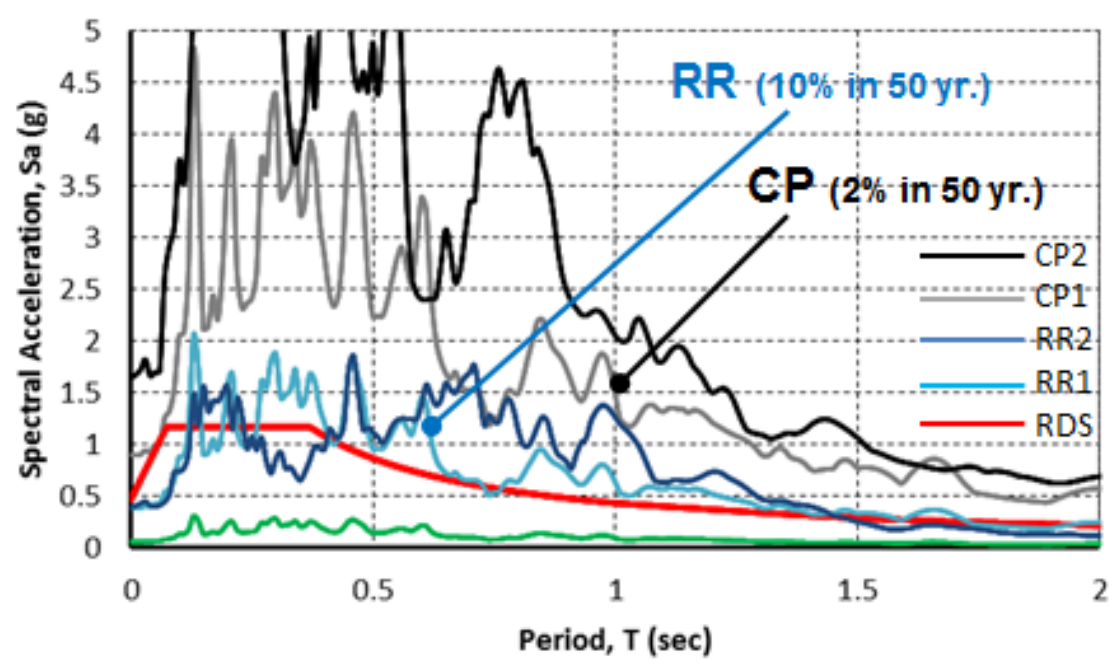

Figure 4.8: Ground motions used in the dynamic analyses. 


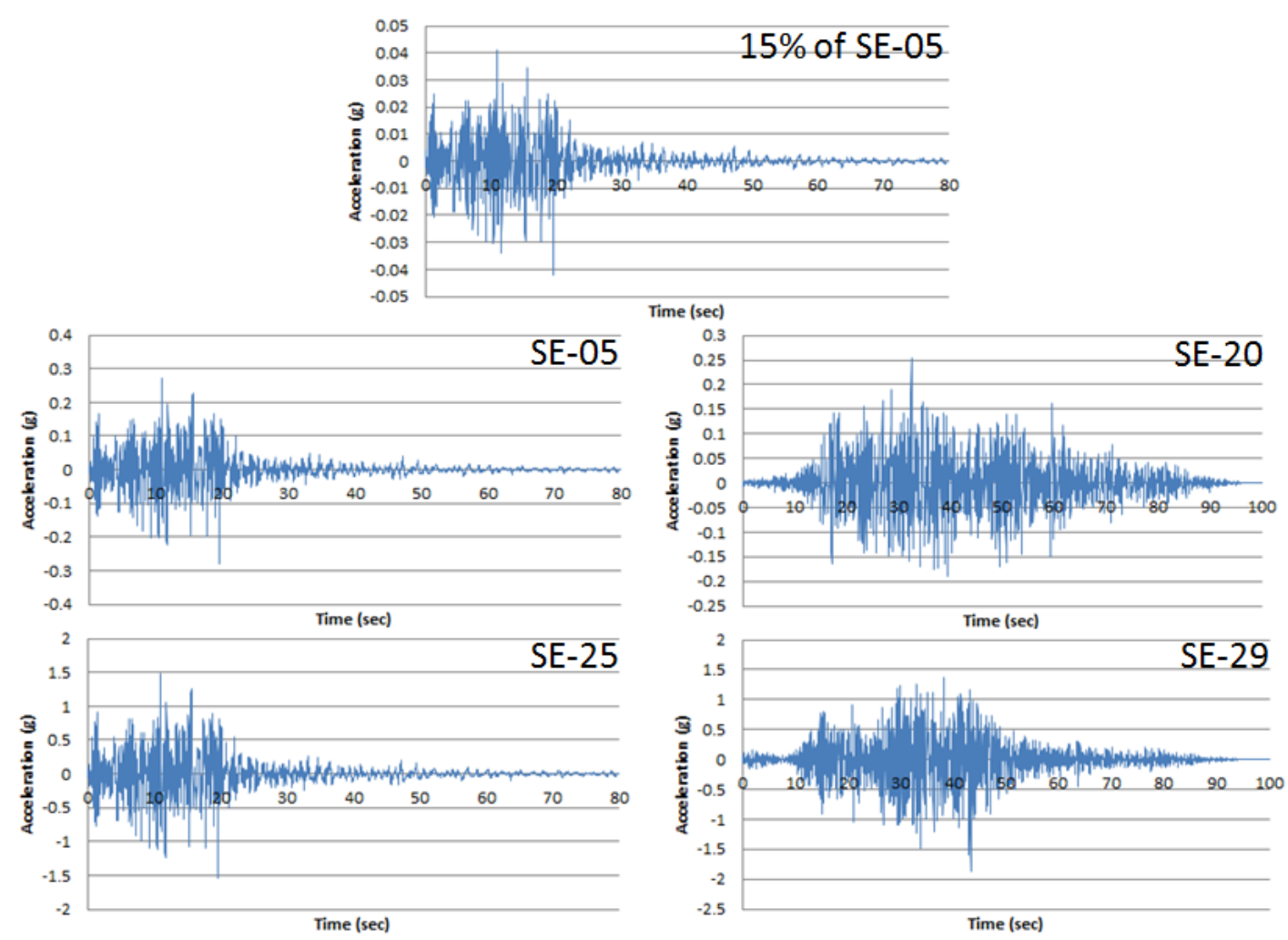

Figure 4.9: Acceleration time history used in the dynamic analyses.

\subsection{LCF Building Response}

The main contribution of this research is to validate a performance based design methodology through hybrid simulation experimental investigation. As such, there was a need to obtain maximum values regarding internal forces (bending moment, shear and axial) in order to proper design the LCF building to be experimentally tested. Three types of analyses were performed: static, nonlinear pushover and time history. Lopes et. al. $\left(2012^{b}\right)$ used for the 2-story and 4-bay LCF building, static and nonlinear pushover analyses to design the specimen that was tested in the laboratory, whereas Malakoutian (2012) worked heavily on 
the numerical aspect of the system using time history analyses. Malakoutian's model was used to confirm the maximum values of the LCF system obtained through the previous analyses mentioned before.

\subsubsection{Equivalent Lateral Force Procedure}

Prior to performing pushover or dynamic time history analyses, a static analysis using the Equivalent Lateral Force (ELF) procedure was performed to verify the design of the LCF building. Since the design strength was approximately verified by pushover analysis and the design of moment frame buildings is generally found to be drift sensitive, this design verification was limited to a drift check.

The seismic base shear $V$ in a given direction is determined in accordance with Equation (8.4) (ASCE/SEI 7, 2010):

$$
V=C_{S} \cdot W
$$

where $C_{S}$ is the seismic response coefficient and $W$ is the effective seismic weight. The seismic response coefficient shall be determined in accordance with Equation (4.2).

$$
C_{S}=\frac{S_{D S}}{\left(\frac{R}{I_{e}}\right)}
$$

where $S_{D S}$ is the design spectral response acceleration parameter in the short period range, $R$ is the response modification factor and $I_{e}$ is the importance 
factor.

The value of $C_{S}$ computed in accordance with Equation 4.2 need not exceed the following:

$$
C_{S}=\frac{S_{D 1}}{T \cdot\left(\frac{R}{I_{e}}\right)}
$$

for $T \leq T_{L}$, and $C_{S}$ shall not be less than

$$
C_{S}=0.044 . S_{D S} \cdot I_{e} \geq 0.01
$$

where $S_{D 1}$ is the design spectral response acceleration parameter at a period of $1.0 \mathrm{sec}, T$ is the fundamental period of the structure, and $T_{L}$ is the long-period transition period.

The values used in these calculations were as follows: $S_{D S}=0.87 \mathrm{~g}, S_{D 1}=$ $0.30 g, T=0.76 s e c, C_{S}=0.108 g, W=2,857 k i p s$, and $V=310 k i p s$. Since the system has 2 grid lines of LCF, the base shear should be divided by 2 . $R=8$ was used as specific LCF system coefficients have not yet been established at the time that these analyses were performed.

The total lateral force was distributed over the height of the building in accordance with the following equations: 


$$
\begin{array}{r}
F_{x}=C_{v x} \cdot V \\
C_{v x}=\frac{w_{x} \cdot h_{x}^{k}}{\sum_{i=1}^{n} w_{i} \cdot h_{i}^{k}}
\end{array}
$$

where $C_{v x}$ is the vertical distribution factor, $w_{i}$ and $w_{x}$ correspond to the portion of the total effective seismic weight of the structure located or assigned to level $i$ or $x, h_{i}$ and $h_{x}$ correspond to the height from the base to level $i$ or $x$, and $k$ is an exponent related to the structure period. $k$ is determined in accordance with the following equation:

$$
k=0.5 . T+0.75
$$

Using the building weight, the vertical distribution of shear was determined as indicated in Table (4.5) and illustrated in Figure (4.10). Should be noted that the accidental torsion was included in the analysis. 
Table 4.5: Details of Seattle seismic forces.

\begin{tabular}{ccccc}
\hline Level & $w_{x}($ Kips $)$ & Sum w(Kips) & $h_{x}(\mathrm{ft})$ & $\mathrm{h}(\mathrm{ft})$ \\
\hline Floor 2 & 1325 & 1325 & 20 & 10 \\
Floor 1 & 1532 & 2857 & 10 & 10 \\
Sum & 2857 & - & - & - \\
\hline \multirow{2}{*}{$w_{x} \cdot h_{x}^{k}(\mathrm{kips}-\mathrm{ft})$} & $\%$ & Fx (Kips) & $\mathrm{Fx}(\mathrm{Kips})$ - torsion \\
\hline & 39118 & 65 & 101 & 106 \\
\hline Sum & 20666 & 35 & 53 & 56 \\
\hline
\end{tabular}

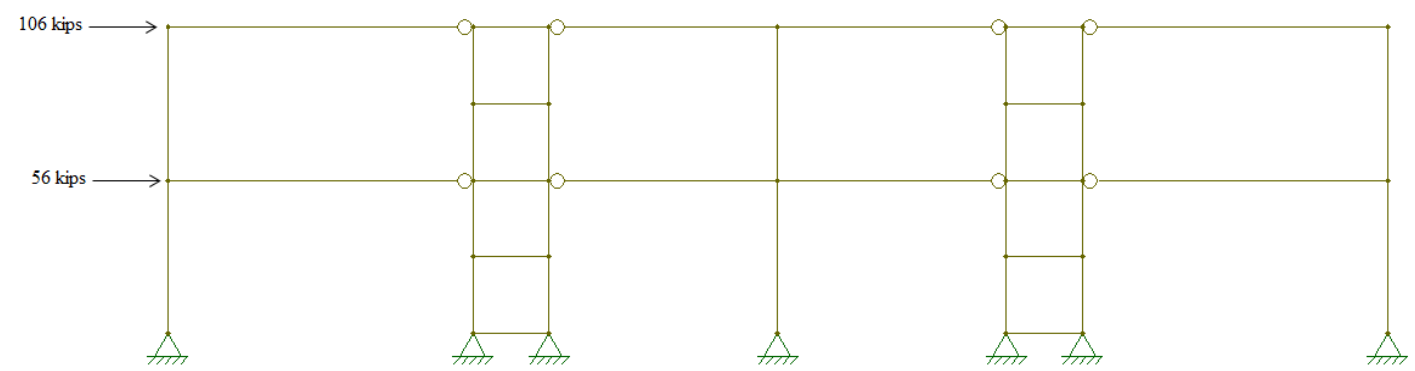

Figure 4.10: Seismic forces used in the ELF procedure.

After applying these lateral loads to the numerical model, the linear displacements $\delta_{x e}$ between each two stories were determined by an elastic analysis. The elastic linear displacements of level $x$ were modified in accordance with the following equation:

$$
\delta_{x}=\frac{C_{d} \cdot \delta_{x e}}{I}
$$

where $C_{d}=5.5$ is the deflection amplification factor. Table (4.6) indicates elastic and design drifts, and drift limit of each story for the structure, while 
Figure (4.11) shows the deformed shape of the structure. Allowable drifts were calculated in accordance with (ASCE/SEI 7, 2010). The final location of the links of LCF system was based upon the drift demands determined from this analysis. Table (4.6) also indicates that the story drift requirements have been met for all stories in the structure for the proposed layout of the lateral systems.

Table 4.6: Drift story check for the 2-story \& 4-bay LCF systems.

\begin{tabular}{cccccc}
\hline LCF-5L & Story height (in) & Elastic Displ. (in) & Elastic drift (in) & Design drift (in) & Drift limit (in) \\
\hline Floor 2 & 110 & 0.55 & 0.26 & 1.43 & 2.75 \\
Floor 1 & 110 & 0.29 & 0.29 & 1.60 & 2.75 \\
\hline LCF-3L & Story height (in) & Elastic Displ. (in) & Elastic drift (in) & Design drift (in) & Drift limit (in) \\
\hline Floor 2 & 110 & 0.74 & 0.34 & 1.87 & 2.75 \\
Floor 1 & 110 & 0.40 & 0.40 & 2.20 & 2.75 \\
\hline
\end{tabular}

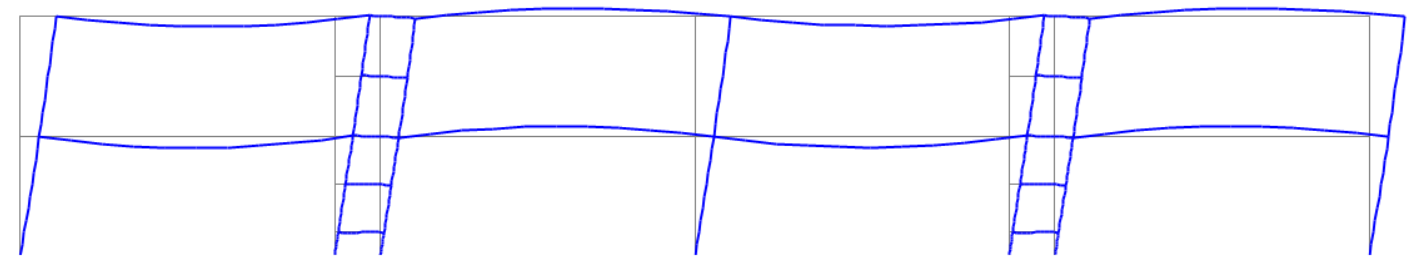

Figure 4.11: Deformed shape for the LCF-5L system.

\subsubsection{Pushover Analysis}

Although the LCF structures were analyzed using the time history approach, nonlinear static analysis (pushover) were conducted using the load pattern from the ELF procedure. The pushover analysis is an evaluation method in which force and displacement demands are estimated from a nonlinear analysis. This 
analysis provides information in regards to the inelastic behavior of the building and potential collapse mechanisms. According to FEMA P-695 (2009), a pushover analysis is a reasonable approach for first mode dominated structures but is not representative of the dynamic response of structures in which higher modes dominate.

Pushover analysis in displacement control was carried out under a lateral load pattern corresponding to the fundamental mode shape to determine the base shear capacity and post-yield behavior on the basis of the building models. The structure was pushed up to $5 \%$ drift. The pushover analysis made use of the Newton algorithm with the convergence tolerance set at $10^{-4}$ and the maximum number of iterations set at 50. A step size of 0.001 in was chosen based on a preliminary study to ensure convergence. In the cases where convergence was a problem, either the Broyden algorithm or the Newton algorithm with line search was used.

Numerical models were designed as per LCF preliminary design procedure described in Chapter (3) to determine the section sizes of LCF systems meeting the design intent of $2.5 \%$ inter-story drift limits. All steel was assumed to be $345 \mathrm{MPa}$ nominal yield stress and the resulting LCF specimen is summarized as follows: gravity columns (W14x132), linked columns (W14x132), gravity beams (W16x57), and replaceable links (W10x45). The LC links have been considered to be short and plastically shear dominated.

Estimate lateral response for a 2-story, 4-bay LCF system is shown in Figure 
(4.12). Individual contributions from LC and MF subcomponents were determined with separate pushover analyses of a modified model where the member connections in MF and LC respectively were released. Points where the first LC link and the first MF beam developed their respective plastic capacity indicated the rapid return to occupancy performance level. The system parameters for a 2-story \& 4-bay LCF and a 2-story \& 1-bay LCF systems are summarized in Table (4.7)

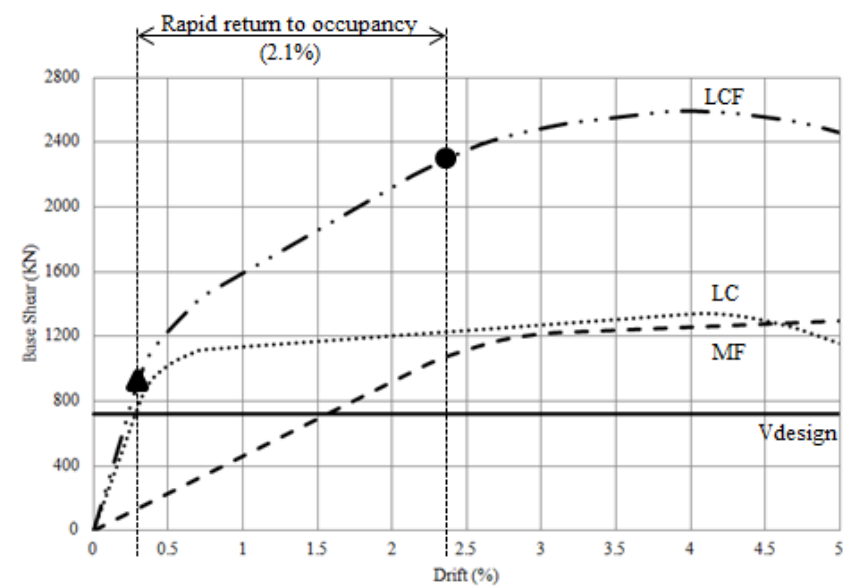

Figure 4.12: Estimate of the lateral response for LCF-5L system.

Table 4.7: LCF system lateral parameters

\begin{tabular}{cccccc}
\hline LCF Type & $\alpha$ & $\beta$ & $\Gamma$ & $\begin{array}{c}\text { First LC } \\
\text { plasticity } \\
\text { drift }\end{array}$ & $\begin{array}{c}\text { First MF } \\
\text { plasticity } \\
\text { drift }\end{array}$ \\
\hline 2-story, 4-bay & 5.55 & 0.79 & 0.14 & 0.30 & 2.40 \\
2-story,1-bay & 10.54 & 1.43 & 0.14 & 0.31 & 2.46 \\
\hline
\end{tabular}

For 2-story buildings the LCs dominated the stiffness as well as strength of the overall structure. Lateral parameters indicated that rapid return to occupancy performance level can occur over a drift range of $2 \%$. Due to differences 
on lateral parameters between the numerical (2-story, 4-bay) and experimental (2-story, 1-bay) models, hybrid simulation was needed to investigate the overall structure response.

\subsubsection{Response History Analysis}

Nonlinear dynamic procedures are required for tall buildings and for buildings with torsional irregularities, but structural member sizes still must be determined before an analysis can be performed. Nonlinear time history analyses were carried out to evaluate the structural response of the LCF systems when subjected to the ground motion described in Section (4.5). Figure 4.13 shows base shear versus drift for the LCF-3L system and Table (4.8) summarizes the expected internal forces during the experiment. 

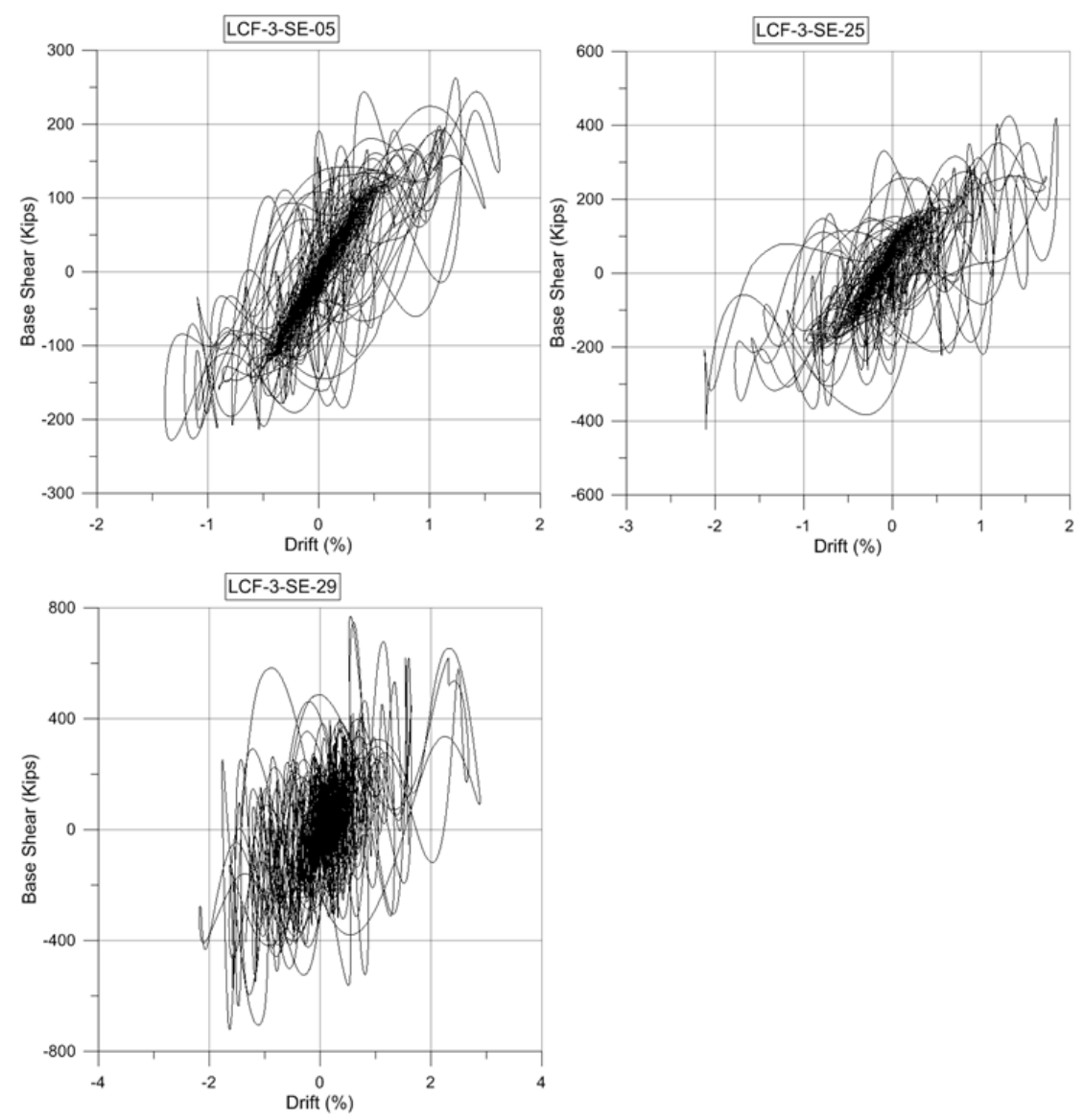

Figure 4.13: Base Shear vs Drift for LCF-3L system.

Table 4.8: Expected internal forces during the experiment.

\begin{tabular}{ccc}
\hline Internal Forces & LCF-5L & LCF-3L \\
\hline GC Axial + & 50 & 55 \\
GC Axial - & 5 & 5 \\
GC Shear & 60 & 50 \\
\hline LC Axial + & 565 & 305 \\
LC Axial - & 645 & 380 \\
LC Shear & 130 & 80 \\
\hline$K_{L C} / K_{M F}$ & 5.55 & 3.77 \\
$V_{L C} / V_{M F}$ & 0.79 & 0.53 \\
$\Delta_{L C} / \Delta_{M F}$ & 0.14 & 0.14 \\
\hline
\end{tabular}

As mentioned before, Malakoutian (2012) worked mainly on the numerical aspect of the LCF building while decisions had to be made in order to experimental test the LCF specimens. As such, the expected internal forces during 
the experiment were obtained through pushover analysis and then compared to time history analysis indicating similar values.

\subsection{Numerical Substructure Component}

Thus far the LCF buildings were numerically analyzed considering either the entire system (2-story \& 4-bay) or the experimental subsystem component (2story \& 1-bay). Due to differences on lateral parameters between system and subsystem responses, hybrid simulation was needed to better understand the behavior of the LCF system. Hybrid testing allows for full scale study at the system level accounting for the uncertainties via experimental component and having the ability to model more conventional behavior through numerical simulation. In order to proceed with the experimental tests in the laboratory, a numerical substructure component was developed using OpenSees in conjuction with OpenFresco. Figure (4.14) shows the numerical substructure component of the LCF system.

The numerical substructure component consisted of a moment frame that connects two LCF subsystems. Masses from the LCF subsystems were transferred to the moment frame and a experimental element was used to attach the numerical and experimental parts. 


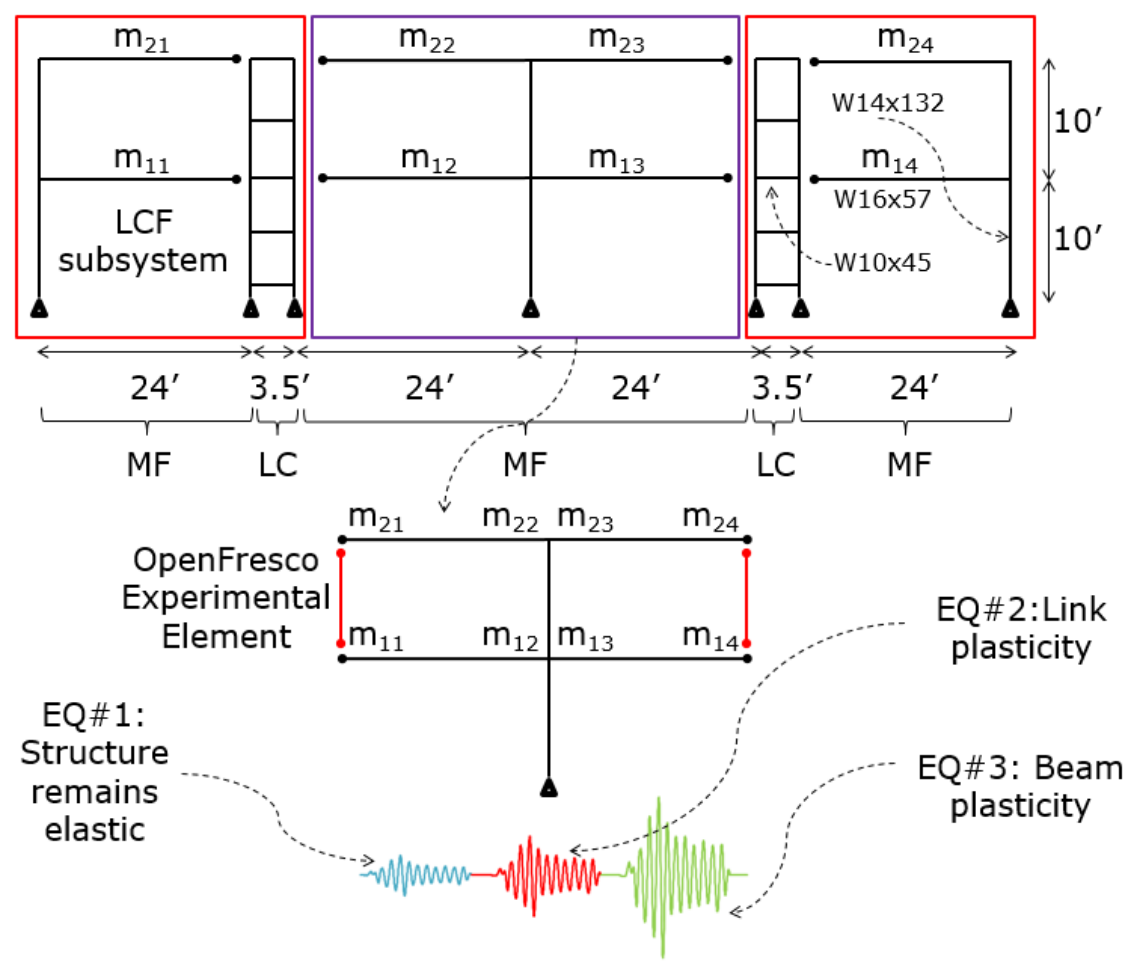

Figure 4.14: Numerical substructure component.

The Tool Command Language, hereafter denoted as tcl script, for the numerical substructure component was created in Notepad ++ and was divided into four parts: main routine, input, solution, and output modules. The main routine represents the skeleton of the tcl script and consists of the main lines of the solution process. This routine calls other routines to perform specific tasks. The input module consists of the geometry of the structure, element, section, material properties, and loads. Information is also needed on the fixity condition of the joints. The element data consist of connectivity, that is, joint numbers and section numbers associated with each element. The section data are required for all the different sections used in the structure and consists of 
cross-sectional area and moment of inertia of the section. Modulus of elasticity and shear modulus are defined on the material data while loaded joints and elements are defined on the load parameters. The solution module is responsible for assembly of the structure matrices (mass, damping and stiffness), assembly of the load vector, solution of the equilibrium equations, and calculations of element-displacement and member-end force vectors. Finally, OpenSees can output a variety of data as defined by the user, which can then be analyzed using a post-processor. The hybrid simulation tcl script is presented in Appendix-A. 


\section{Chapter 5}

\section{Experimental Program}

\section{$5.1 \quad$ Introduction}

The system experiments were moved from the University of Nevada at Reno in 2011 (Lopes et. al., 2010) to the University of California at Berkeley in 2012 (Lopes et. al., $2012^{c}$ ) due to schedule conflicts. The first LCF specimen arrived at the laboratory in November of 2012, whereas the last experimental was conducted in July of 2013. Meanwhile, several trips from Portland-OR to Berkeley-

CA were arranged. All experimental tests were done during spring/summer of 2013 and the LCF system was investigated as a full-scale 2-story and 1-bay structure. Cyclic and hybrid simulation tests were performed. The large scale nees@berkeley structure laboratory is equipped with a state-of-the-art servohydraulic system for simulating earthquake loads. The laboratory features a main test floor, a configurable reaction wall, bridge cranes, hydraulic actuators, digital controllers, and a data-acquisition system with a variety of instrumentation devices. The analyses thus far have focused on system level numerical 
model development. The next step in understanding the behavior of the system was to perform experimental testing on a typical LCF system. The goals of the tests were to understand how the LCF system components interact together as a unit, to monitor the progression of damage in the replaceable links and gravity beams, and ultimately validate the LCF system.

\subsection{Specimen Design}

\subsubsection{Specimen Dimensions}

The design resistance of the LCF was based on a prototype building that was a modified version of the 3-story building SAC configuration. The modification was due to physical space laboratory constraints. In elevation, the typical bay width was $24.5 \mathrm{ft}$ and typical story height was $10 \mathrm{ft}$ and each LC was spaced $3.5 \mathrm{ft}$ apart. The two specimens denoted as LCF-5L (five links) and LCF-3L (three links) are shown in Figure (5.1).

The member sizes and dimensions of the test specimens were chosen to approximate large scale frame for a two story building constructed in a medium to high seismic design category and to meet the constraints of the laboratory. The laboratory constraints included: (1) capacities of the actuators and strong floor, (2) height of the reaction wall and (3) plan dimensions of the strong floor. The design of the frame members are discussed in Section (5.2). Detailed design calculations for the specimen and structural drawings, that were supplied to the steel fabricator, are available in Appendices B and C, respectively. 


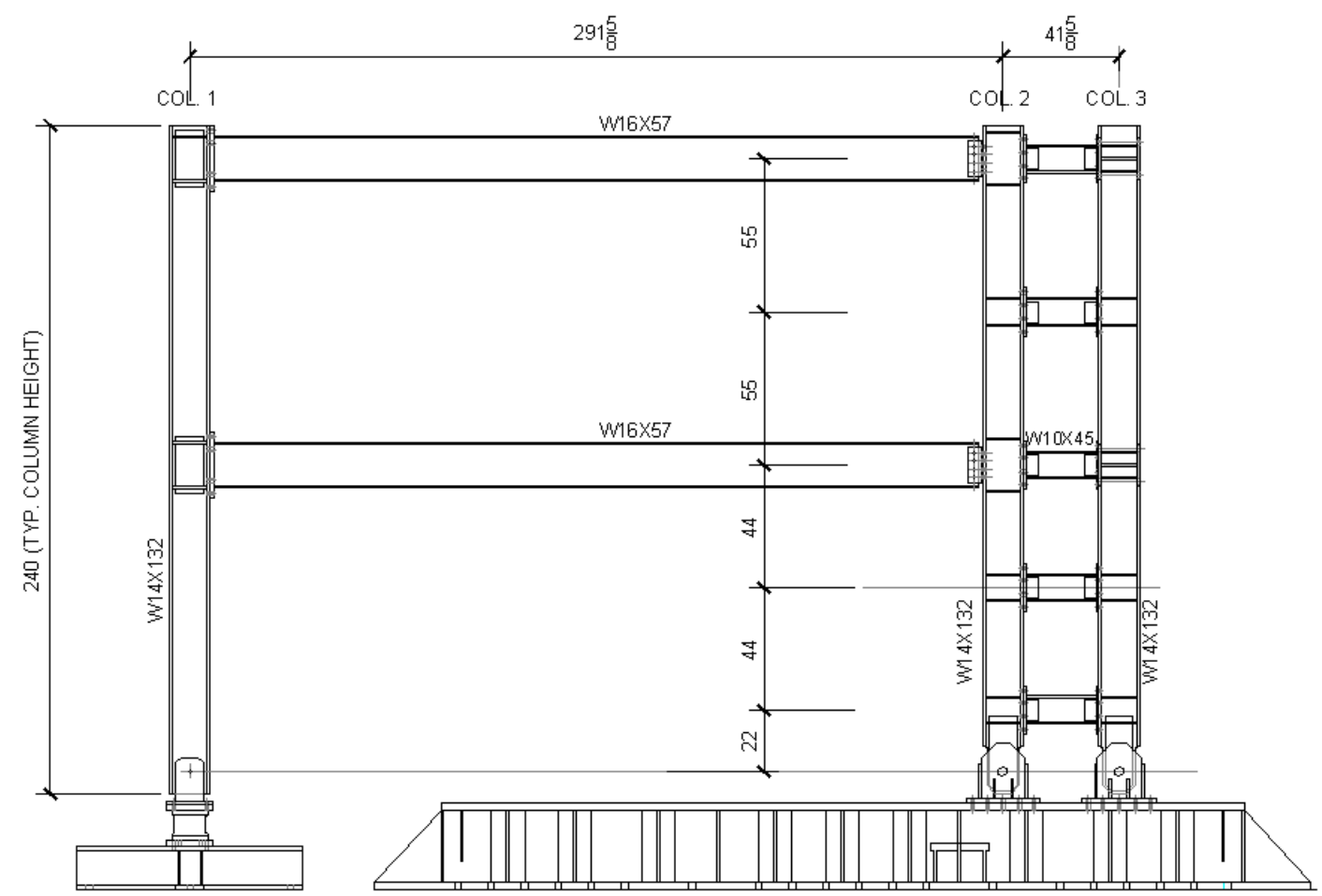

(a) LCF-5L

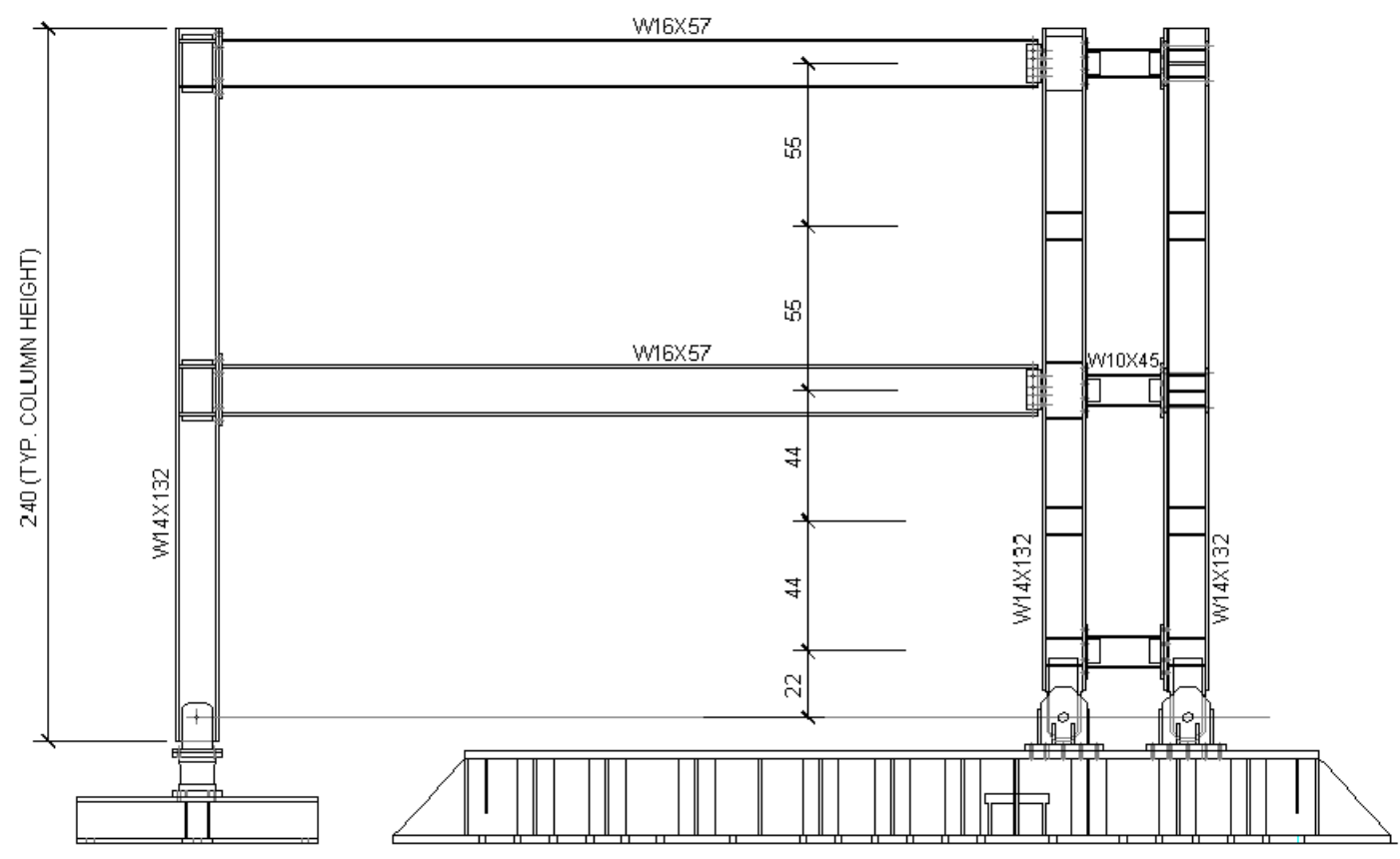

(b) LCF-3L

Figure 5.1: Elevation of the LCF specimens. 


\subsubsection{Beams and Columns}

The LCF system was designed for strength and ductility. Strength is related to the maximum capacity of the structural member to resist a specific load and ductility is related to the maximum deformation beyond the yield stress without loss of strength. The beams and columns were designed according to capacity principles and additional demands from structural analysis. The demands from gravity load were relatively small due to the inability to apply gravity load to the floor system in the laboratory. Therefore, the gravity demands on the system were only from the self weight of the structure.

For doubly symmetric wide flange members bent about its strong-axis the applicable LRFD flexural strength limit states are yielding and lateral-torsional buckling (LTB). When the member unbraced length, $L_{b}$, is less than the limiting laterally unbraced length for the limit state of yielding, $L_{p}$, the LTB limit state does not apply and flexural strength is determined by the plastic moment capacity, $M_{p}$, of the section given by Equation (5.1). When $L_{b}$ is between $L_{p}$ and the limiting laterally unbraced length for the limit state of inelastic LTB, $L_{r}$, the flexural capacity is determined using Equation (5.2).

When $L_{b} \leq L_{p}$

$$
\phi_{b} M_{n}=\phi_{b} F_{y} Z_{x}
$$

When $L_{p}<L_{b} \leq L_{r}$

$$
\phi_{b} M_{n}=\phi_{b} C_{b}\left[M_{p}-\left(M_{p}-0.7 F_{y} S_{x}\right)\left(\frac{L_{b}-L_{p}}{L_{r}-L_{p}}\right)\right] \leq \phi_{b} M_{p}
$$

where $C_{b}$ is the lateral-torsional buckling modification factor, $F_{y}$ is the yielding stress, $M_{n}$ is the nominal flexural strength, $S_{x}$ is the elastic section modulus about the x-axis, $Z_{x}$ is the plactic section modulus about the x-axis, and $\phi_{b}$ is the resistance factor for flexure. For the W16x57 section the value of $L_{p}$ was 
$5.7 \mathrm{ft}$ and $L_{r}$ was $18.3 \mathrm{ft}$. Since $L_{b}=24.5 \mathrm{ft}$ is greater than $L_{r}$, the strength was based on elastic LTB.

The LRFD axial compressive strength of doubly symmetric wide flange members is governed by the flexural buckling limit state. Compression strength is controlled by the member slenderness ratio $(K L / r)$. The effective length factor, $K$, accounts for member end conditions. The design compressive strength is given by the following equations:

$$
\phi_{c} P_{n}=\phi_{c} F_{c r} A_{g}
$$

$$
\text { When } \frac{K L}{r} \leq 4.71 \sqrt{\frac{E}{F_{y}}}
$$

$$
F_{c r}=\left[0.658^{\frac{F_{y}}{F_{e}}}\right] F_{y}
$$

$$
\text { When } \frac{K L}{r}>4.71 \sqrt{\frac{E}{F_{y}}}
$$

$$
\begin{gathered}
F_{c r}=0.877 F_{e} \\
F_{e}=\frac{\pi^{2} E}{\left(\frac{K L}{r}\right)^{2}}
\end{gathered}
$$

where $A_{g}$ is the gross area of the member, $E$ is the modulus of elasticity, $F_{c r}$ is the critical stress, $F_{e}$ is the elastic critical buckling stress, $L$ is the length of member, $r$ is the radius of gyration, and $\phi_{c}$ is the resistance factor for compression. According to Galambos (1998) these column strength equations consider the influence from initial residual stress and geometric imperfections.

The structural analysis for design was performed with both OpenSees (McKenna, 1997) and SAP 2000 (SAP2000, 2010) by pushing the test specimen model out to $5 \%$ drift, with the results being similar. The internal forces and corresponding displacements were found from the above structural analysis. When a column is subjected to combined axial and flexural loading, a portion of the member 
capacity is consumed by the axial force demand and a portion by the moment demand. An interaction equation, that is dependent on the ratio of axial force demand to capacity, was used to evaluate whether or not the member has adequate strength for the imposed demand. Before evaluating member strength for combined loading the independent axial and flexural capacities were determined. The demand capacity ratios of the beams and columns were determined according to the following AISC (2011) equations:

For $P_{u} / \phi P_{n}>0.2$

$$
\frac{P_{u}}{\phi P_{n}}+\frac{8}{9} \cdot\left(\frac{M_{u x}}{\phi M_{n x}}+\frac{M_{u y}}{\phi M_{n y}}\right) \leq 1.0
$$

For $P_{u} / \phi P_{n}<0.2$

$$
\frac{P_{u}}{2 \phi P_{n}}+\left(\frac{M_{u x}}{\phi M_{n x}}+\frac{M_{u y}}{\phi M_{n y}}\right) \leq 1.0
$$

During an earthquake the structure is designed so it can provide energy dissipation as well. Therefore, the structure is designed so it can develop inelastic structural behavior. This behavior is achieved when the column members remain elastic providing strength and stability of the stories above and the beam members behave plastically. Frames in which measures are taken to promote plastic hinges in the beams rather than in the columns are said to be Strong Column and Weak Beam (SCWB) frames. The behavior of a steel beam is more ductile than a steel column, so it is safer to establish the location of the plastic hinges at the end of the beam rather at the column. The philosophy behind this approach is that, at a joint, the summation of the columns' plastic moment capacities exceed the summation of the beam's plastic moment capacities, based 
on simple moment equilibrium at the joint as defined by the ratio:

$$
S C W B=\frac{\sum M_{p c}}{\sum M_{p b}} \geq 1.0
$$

where $\sum M_{p c}$ is the summation of the projections to the beam centerline of the nominal flexural strengths of the columns above and below the joint and $\sum M_{p b}$ is the summation of the expected flexural strengths of the plastic hinges in the beams, projected from the hinge location to the column centerline. For the strong column and weak beam check, several cases were analyzed and two of them are summarized below:

1. Joint at gravity column and gravity located at first floor level, where SCWB was equal to 1.63.

2. Joint at linked column and first shear link located at foundation level, where SCWB was equal to 1.17.

The above requirement cannot fully prevent column plastic hinges at beamto-column joints because the internal forces acting at the top and bottom faces of a joint varies greatly during an earthquake. It is believed that satisfying Equation (5.9) will limit column yielding, resulting in columns strong enough to spread beam plastic hinges over multiple frame levels.

The panel zones of the frame columns were analyzed to determine if the webs were adequately sized to prevent yielding according to the current philosophy used for moment frames ANSI/AISC 341 (2010). The check was performed as a basis for assessing the behavior of panel zones of the frame columns where column stiffeners were an option due to a beam joint. The forces on the panel zones were determined assuming that the beams formed plastic hinges at a distance of $d / 2$ from the face of the column. The column shears were determined 


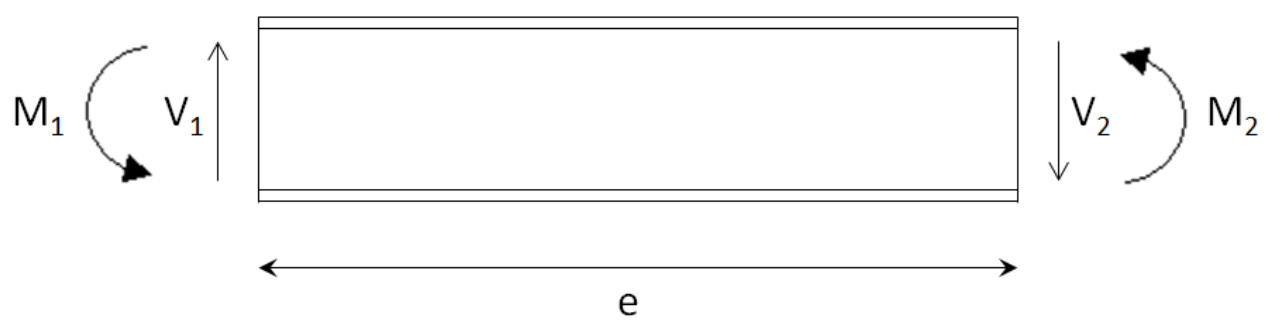

Figure 5.2: Free-body diagram of a link.

from equilibrium. The plastic moment at the beam face was determined ignoring axial load in the beam.

\subsubsection{Shear Links}

Shear links were necessary to interconnect the dual columns and guarantee a controlled drift behavior. Figure (5.2) shows the free-body diagram of a link. If axial force and moment-shear interaction are ignored, plastic flexural hinges will form at nodes 1 and 2 of the link when moments $M_{1}$ and $M_{2}$ reach plastic moment, $M_{p}$. A plastic shear hinge is formed when shear reaches $V_{p}$. The plastic moment and plastic shear are respectively computed as follows:

$$
\begin{gathered}
M_{p}=F_{y} \cdot Z_{x} \\
V_{p}=0.6 \cdot F_{y} \cdot A_{w}
\end{gathered}
$$


where $A_{w}$ is the link web area. If $M_{p}$ and $V_{p}$ are reached at the same time, the corresponding link length is given by:

$$
e_{0}=\frac{2 \cdot M_{p}}{V_{p}}
$$

where $e_{0}$ is the link length when a balanced yielding condition is achieved.

According to Bruneau et. al. (1998) test results showed that a properly stiffened shear link can strain harden and develop a shear strength equals to $1.5 V_{p}$. Also, to avoid high bending strains that may lead to severe flange buckling or to failure of link flange-to-column welds, these end moments are limited to $1.2 M_{p}$, and the maximum length, $e_{0}$, in Equation (5.13), for a shear link is modified as follows Kasai \& Popov (1986).

$$
e_{0}=\frac{2\left(1.2 M_{p}\right)}{1.5 V_{p}}=\frac{1.6 M_{p}}{V_{p}}
$$

Links in the LCF specimens were designated as structural fuses to dissipate energy and were designed to satisfy several conditions simultaneously such as desired shear strength, link length limit, link rotation limit, and inter-story drift limit, while meeting the seismic limits for compactness. Members of the LCF system shall have flanges continuously connected to the web and the widththickness ratios of its compression elements shall not exceed the limiting ratios 
below.

$$
\begin{aligned}
\frac{b_{f}}{2 t_{f}} & =0.30 \frac{E}{F_{y}} \\
\frac{h}{t_{w}} & =2.45 \frac{E}{F_{y}}
\end{aligned}
$$

The nominal shear strength of a link, denoted $V_{n}$, is the lesser of $V_{p}$ and $2 M_{p} /$ e. Also, the design shear strength of a link is given by the following equation

$$
V_{r}=0.9 V_{n}
$$

According to ANSI/AISC 341 (2010) the link rotation angle shall not exceed 0.08 radians for links of length $1.6 M_{p} / V_{p}$ or less. Therefore, the plastic moment considering the link rotation angle for a shear link can be written as

$$
M_{p(r o t)}=\frac{V_{p} \cdot e}{1.6}
$$

Because there are two limit states, both of the following equations must be satisfied, where $V_{u}$ and $M_{u}$ are values from structural analysis.

$$
\begin{gathered}
V_{u}<V_{r} \\
M_{u}<M_{p_{(r o t)}}
\end{gathered}
$$

Considering a yield stress of $50 \mathrm{ksi}$, a W10x45 shear link cross-section was 


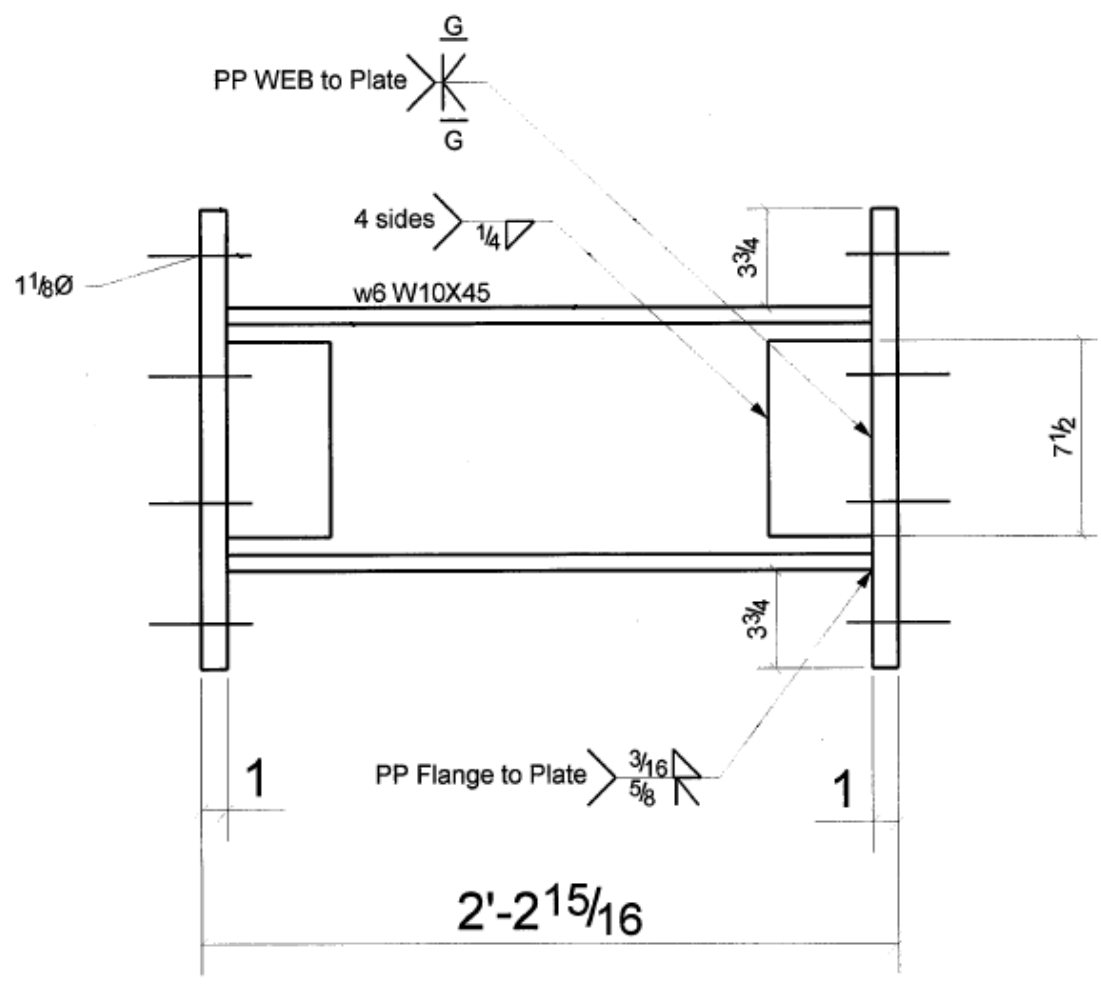

Figure 5.3: Shear link detailing.

used with the following parameters: $d=10.1 \mathrm{in}, t_{w}=0.35 \mathrm{in}, t_{f}=0.62 \mathrm{in}$, $h=8.86 \mathrm{in}, A_{w}=3.1 \mathrm{in}^{2}$, and $Z_{x}=54.01 \mathrm{in}^{3}$. Using these values, the anticipated plastic shear, nominal shear, plastic moment, and plastic moment considering the link rotation were $V_{p}=93$ kips, $V_{r}=84$ kips, $M_{p}=2,700$ kips.in, and $M_{p_{(r o t)}}=1,100$ kips.in. Five links for the first specimen and three links for the second specimen with identical geometric and material properties were fabricated. The duplicate specimens were necessary to access the replaceability of the links. Shear links used on the LCF test assembly and shear link connection are shown in Figures (5.3) and (5.4), respectively.

The shear links were shop welded to an end-plate and then field bolted 


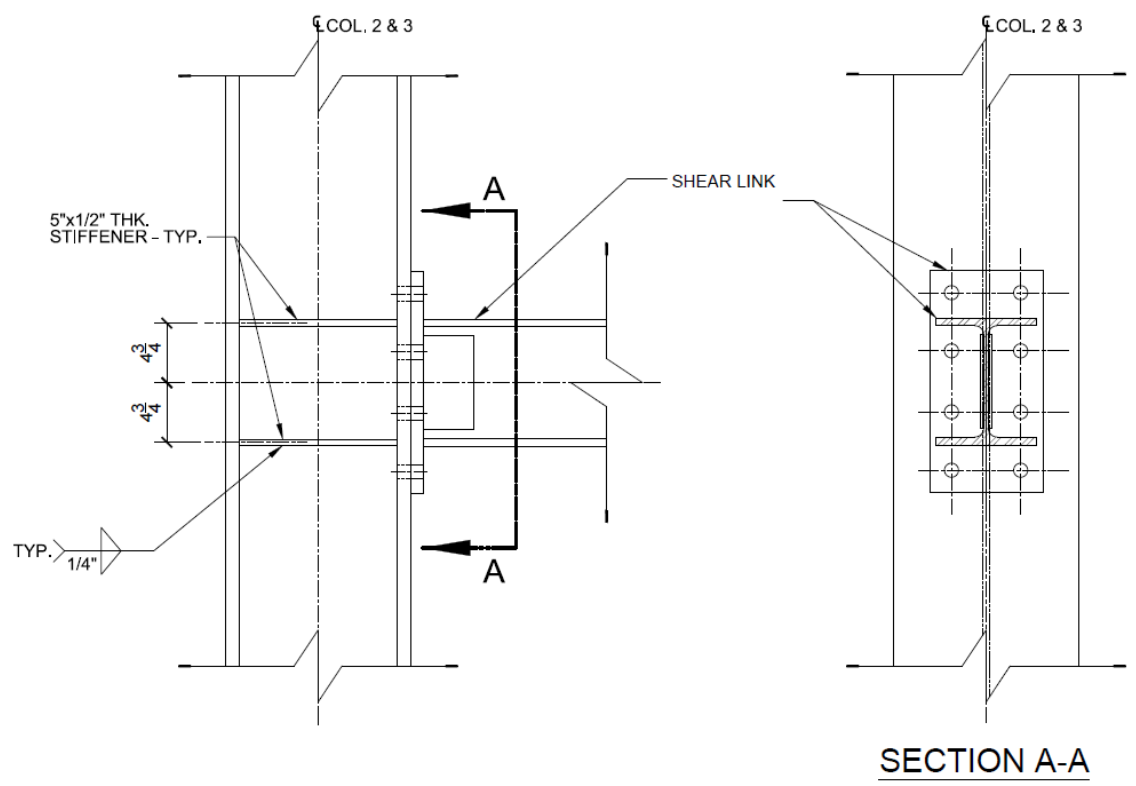

Figure 5.4: Shear link connection.

to the column flange using prestressed high strength bolts. The total length considering the end plates was 27 inches with an effective link length equals to 17 inches. The calculated number of intermediate link stiffener was equal to 1 and spacing equals to $9.5 \mathrm{in}$, however, intermediate link stiffeners were not considered in the design process. It is believed that ANSI/AISC 341 (2010) has an overdesign intermediate link stiffener equation, because the yielding link is coupled with the beam floor in the current design of EBFs. This drawback can be mitigated by designing the system with replaceable shear links and, therefore, reducing the number of intermediate stiffeners.

A partial penetration bevel butt weld (both sides) was chosen to join the web to the end plate connection. As shown in Figure (5.3), the welding flanges were designed with both bevel groove weld (arrow side) and fillet weld (other side). Also, fillet welds were used to join the $3 / 8$ " web to both web and end 
plate connection. The links were connected to the linked columns by eight 1" diameter A490-X bolts.

\subsubsection{Frame Connections}

In order to expedite the construction process and to eliminate the need for field welding, bolted connections were used as framing connections. The connection between gravity beams and gravity column, as well as the connection between links and dual columns were bolted unstiffened extended end-plate moment connections, denoted hereafter as moment connection. The connection between gravity beams and the linked column were bolted shear connections known as a double angle connection, denoted hereafter as shear connection, which were made with two angles, one of each side of the beam to be supported.

Extented end-plate connections were investigated elsewhere as an alternative to welded connections during the aftermath of the Northridge Earthquake, and two variations were chosen as pre-qualified connections: the four bolt unstiffened end-plate connection and the eight bolt stiffened end-plate connection. Design procedures for these two connections subjected to cyclic loading were developed by Sumner \& Murray (2002) and were included in FEMA 350 (2000).

Design procedures used for the moment connection are presented in AISC (2011) and Murray \& Sumner (2004). The two main limit states which control the design, for moment connection, are end plate flexural yielding and bolt tension rupture. However, the following limit states were also considered in the 
design of the moment connection: shear yielding, shear rupture, web buckling, web crippling, and plate buckling. Since most of the moment transferred from the gravity beam to the gravity column results in large concentrated forces, the use of column stiffeners and continuity plates were necessary to reinforce the columns.

For the shear connection, AISC (2011) considers axial load acting on the gravity beam, was used. The following limit states were considered in the design of the shear connection: shear yielding, shear rupture, flexural yielding, flexural rupture, block shear rupture, bolt bearing, bolt shear, yielding under combined shear and tension, rupture under combined shear and tension, block shear under combined shear and tension, and local buckling for the angles.

The gravity beams were shop welded to an end-plate and then field bolted to the column flange using prestressed high strength bolts. The moment and shear connections are shown in Figures (5.5) and (5.6), respectively.

\subsubsection{Column Base Plates and Clevises}

The design of the base column required consideration of bearing pressure on the supporting material and bending of the plate. According to Thornton (1990) there is no need to determine whether the plate is large or small, lightly loaded, or heavily loaded. Thornton (1990) proposed a unified procedure resulting in Equation (5.20) and this procedure was used in order to determine the thickness of the base plate for the LCF columns. 

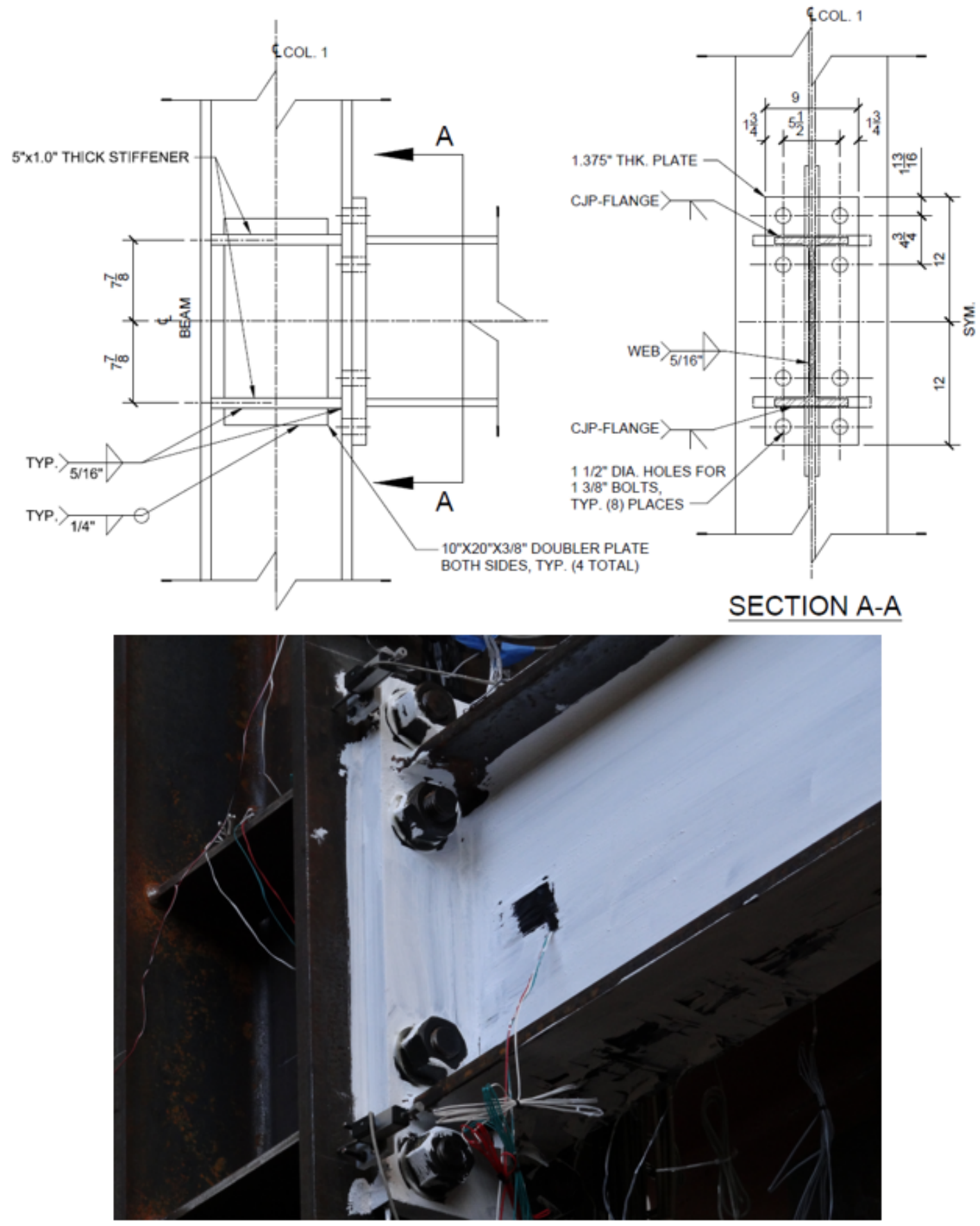

Figure 5.5: Extended-end plate moment connection. 

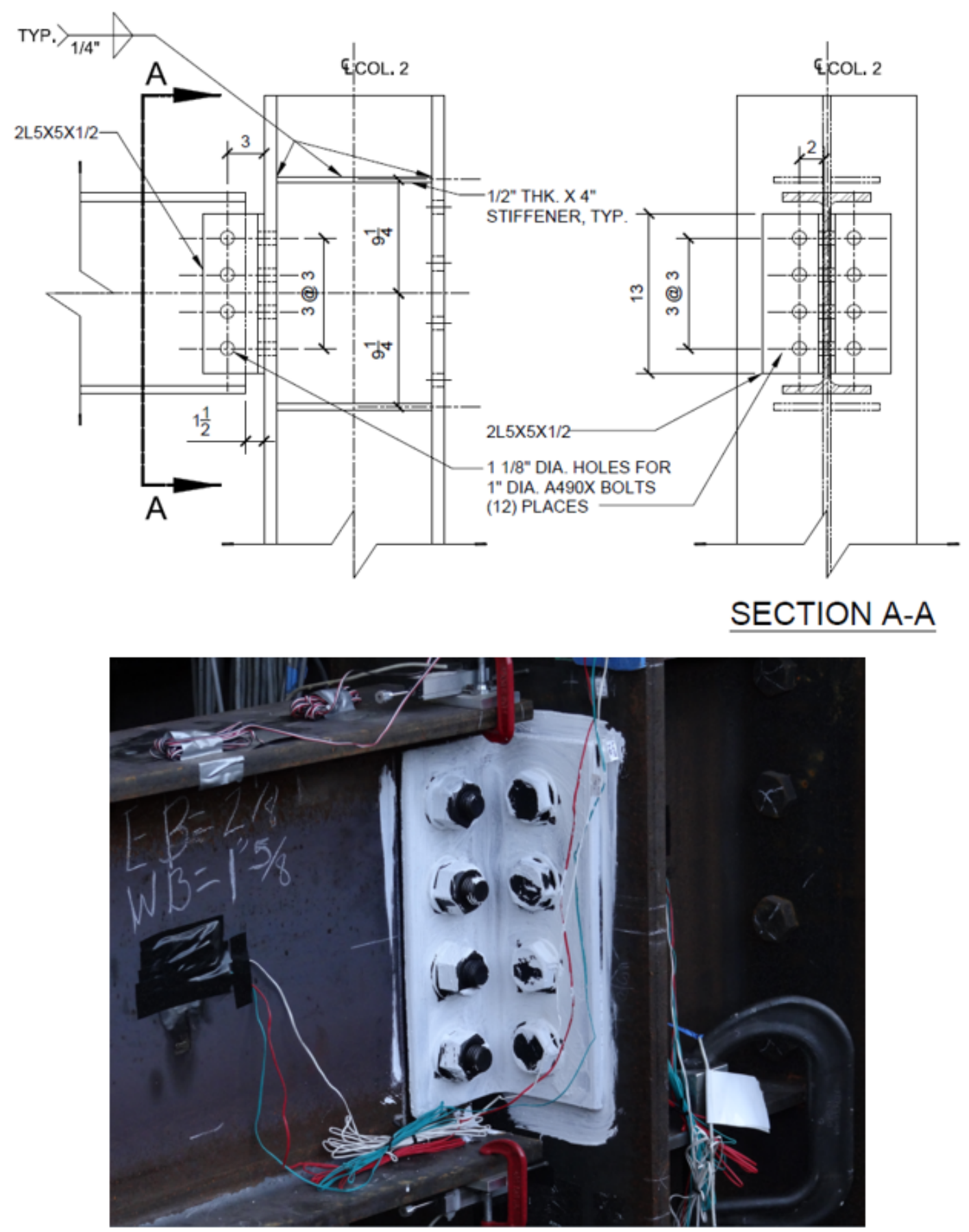

Figure 5.6: Double angle shear connection. 


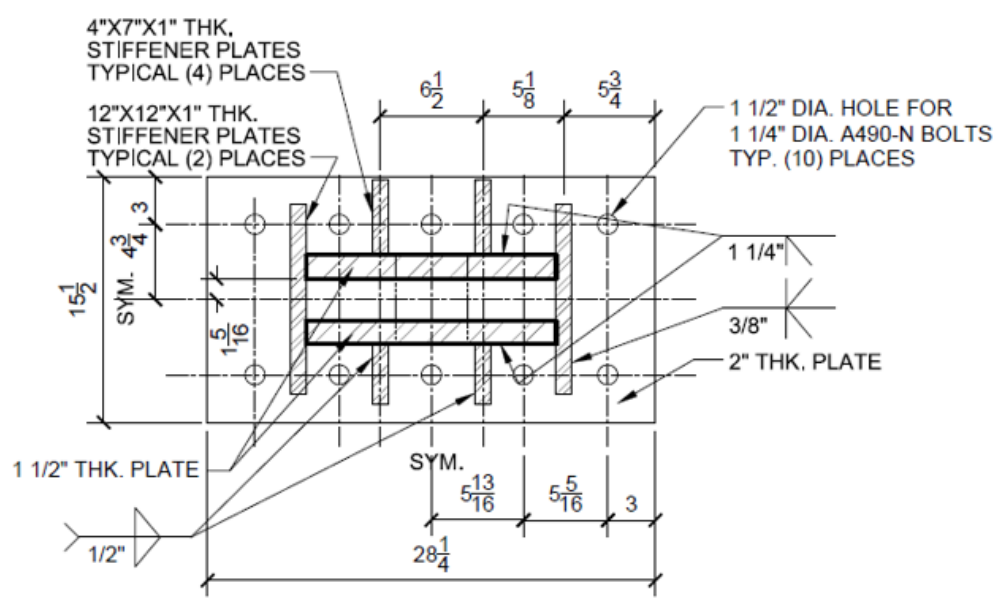

(a) Linked column base plate

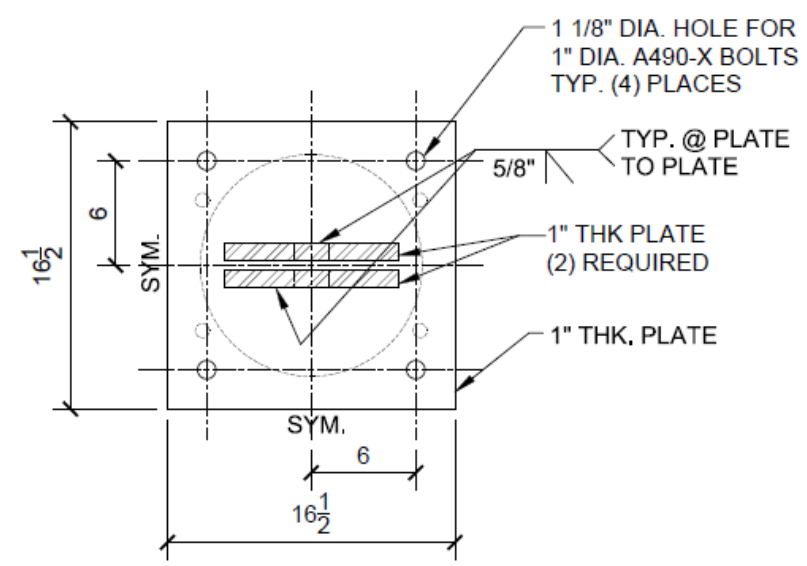

(b) Gravity column base plate

Figure 5.7: (a) Linked column base plate; (b) Gravity column base plate.

$$
t \geq L \sqrt{\frac{2 P_{u}}{0.9 F_{y} B N}}
$$

where $L$ is the maximum cantilever strip, $P_{u}$ is the factored load, $B$ and $N$ are the base plate dimensions. This procedure is the same as that given in Part 14 of the Manual, Design of Beam Bearing Plates, Column Base Plates, Anchor Rods, and Column Splices (AISC, 2011). Figure (5.7) shows the details of these base connections for each column along with the bolt layouts and plate sizes. 


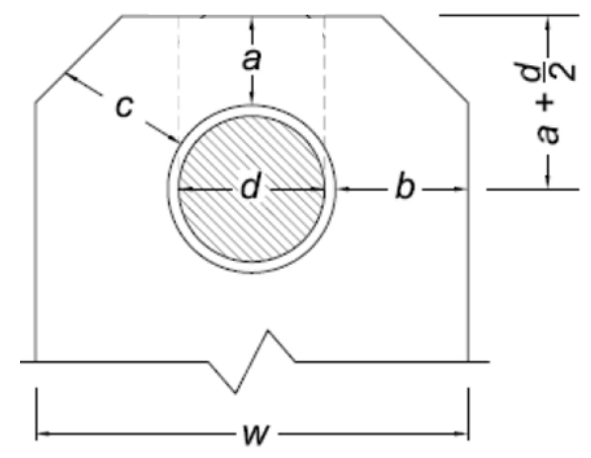

Figure 5.8: Dimensional requirements for pin-connected members.
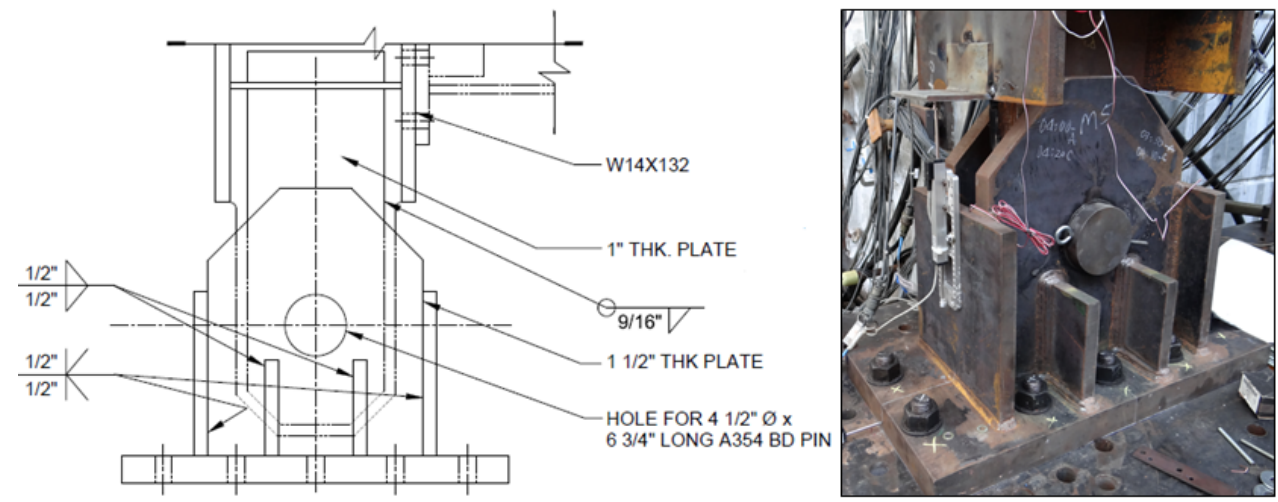

Figure 5.9: Linked column clevis.

Column base plates and clevises were designed to provide an idealized pinned connection that would prevent slip and uplift and also to avoid plastic hinges on the columns. In regards to the pinned connection, the dimension requirements presented in Specification Section D5.2 (AISC, 2011) were met to provide for the proper functioning of the pin. Those requirements are illustrated in Figure (5.8) and are as follows: $a \geq \frac{4}{3} b_{e f f}, w \geq 2 b_{\text {eff }}+d$, and $c \geq a$, where $b_{\text {eff }}=2 t+0.625$. Figure (5.9) and (5.10) show the details of the clevis connections for linked and gravity columns, respectively. 


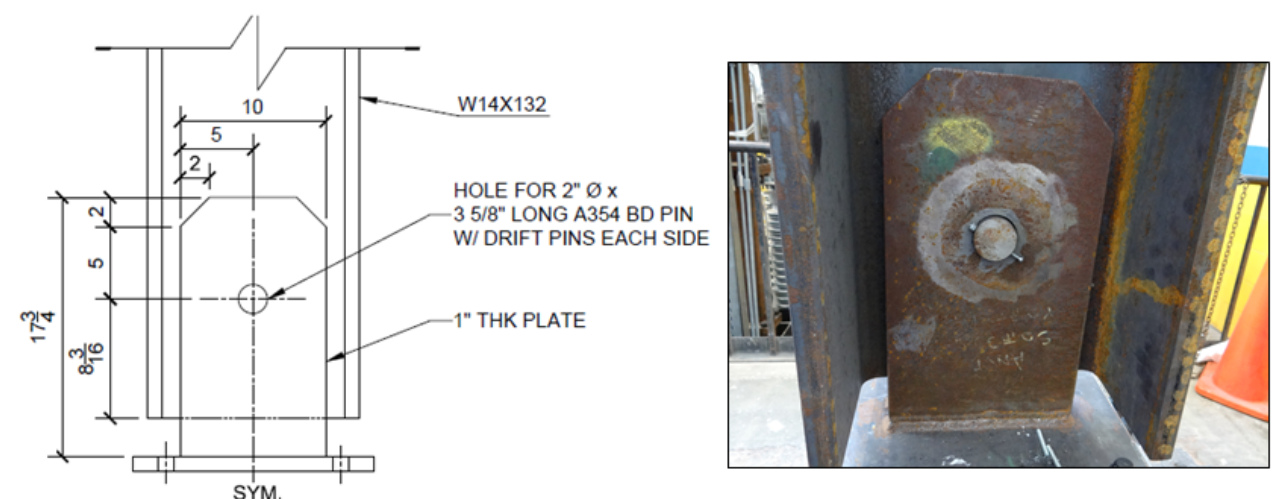

Figure 5.10: Gravity column clevis.

\subsubsection{Summary of Design Characteristics}

The columns and beams of the test specimen were specified to be ASTM A572 Grade 50 steel. Also, plates for column stiffeners, doubler plates and clevises were specified to be ASTM A572 Grade 50 steel. The yield stress for the columns was $60 \mathrm{ksi}$ whereas for the beams was $55 \mathrm{ksi}$. The gravity beam plastic moment, $M_{p}$, was determined equal to 5,775 kips-in. Pin clevises were specified to be ASTM A354 Grade BD steel pin. The links of the test specimen were specified to be ASTM A572 Grade 50 steel. Similarly, all plates for the shear links were specified to be ASTM A572 Grade 50 and compliance was verified by review of the mill certificates. The yield stress for the links was 53 ksi and the plastic shear, $V_{p}$, and plastic moment, $M_{p}$ were equal to 100 kips and 2,625 kips-in, respectively. Bolts were specified as ASTM A490 structural bolts and were pre-tensioned using a hydraulic torque wrench. The turn-of-the-nut method described in AISC (2011) was used for this purpose. Certified mill test and weld inspection reports are presented in Appendix D. 


\subsection{Experimental Test Setup}

The LCF system was tested using parts of an existing test setup already found at nees@berkeley. Prior to the LCF tests, Lai (2012) tested conventional and hybrid braced frames using two actuators attached to the reaction wall. The existing test setup had to be modified in order to accommodate the LCF system. Fours actuators and two new actuator brackets were needed for the LCF project as well as an extra foundation element capable of receiving the gravity column. Modifications on the out-of-plane restraint system and reinforcement on the strong floor were also needed.

\subsubsection{Construction Sequence}

Test specimens were designed at PSU and fabricated by AMT Metal Fabricators, Inc. of Richmond, California and all welding was performed according to AWS (2004) D1.1 prequalified welds. Prior to the arrival of the LCF specimen, several tasks were done in order to properly accommodate the experimental setup. A heavy built-up floor beam, denoted hereafter as LC floor beam, was positioned in place in order to receive the linked columns. Steel brackets made of W14x211 sections were installed on the existing reaction wall to attach the actuators while three HSS columns, which belongs to the out-of-plane system, were also positioned on the strong floor and attached to an existing cantilever beam fixed to the reaction wall. Actuator brackets and out-of-plane system are discussed 


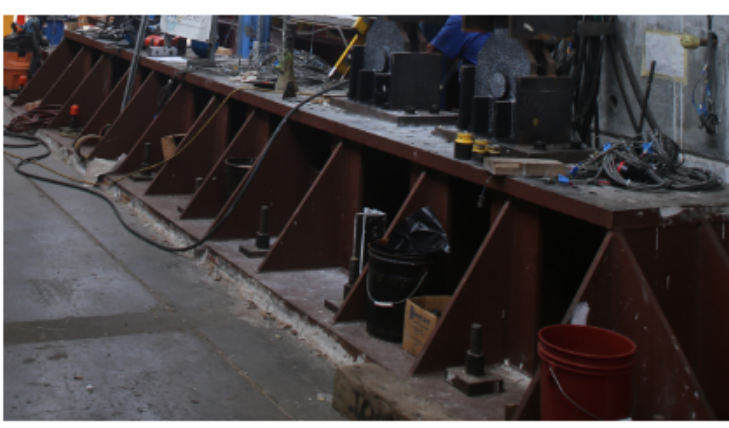

(a) LC floor beam

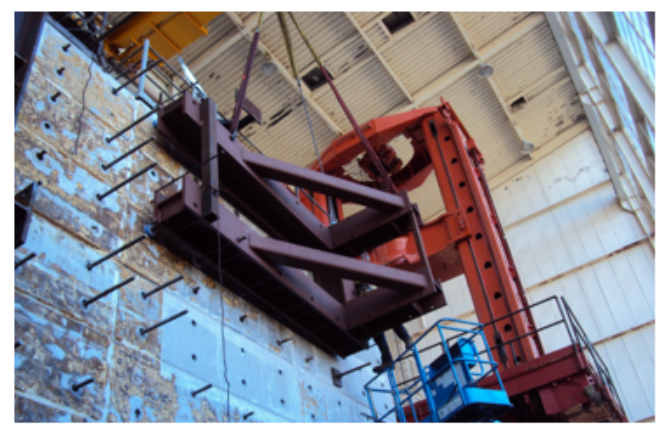

(b) Actuator bracket

Figure 5.11: (a) LC floor beam; (b) Actuator bracket.

in Sections (5.3.2) and (5.3.4), respectively. Figure (5.11) shows the LC floor beam and the upper actuator bracket being installed on the reaction wall.

The LCF-5L specimen was erected within a three-week period in the laboratory. The arrived specimen consisted of a set of linked columns with five links already assembled, two gravity beams and one gravity column. The linked columns with five links were placed on the LC floor beam. Link end-plate connections were shop welded using partial joint penetration at flange to plate and single pass fillet welds at webs. In practice, the links and surrounding components are intended to be welded in the shop and bolted on site, thus there was no need to simulate field welding conditions on the LCF system.

The base plate of the linked columns was attached to the LC floor beam located at the top of the strong floor slab, and a series of relatively stiff load transfer beams were provided on the bottom side of the strong floor slab. The 2 " thick linked column base plates were bolted to the LC floor beam with 1 1/4" $\phi$ A490 bolts. Figure (5.12) shows the linked columns of the LCF-5L specimen being installed on the LC floor beam. 


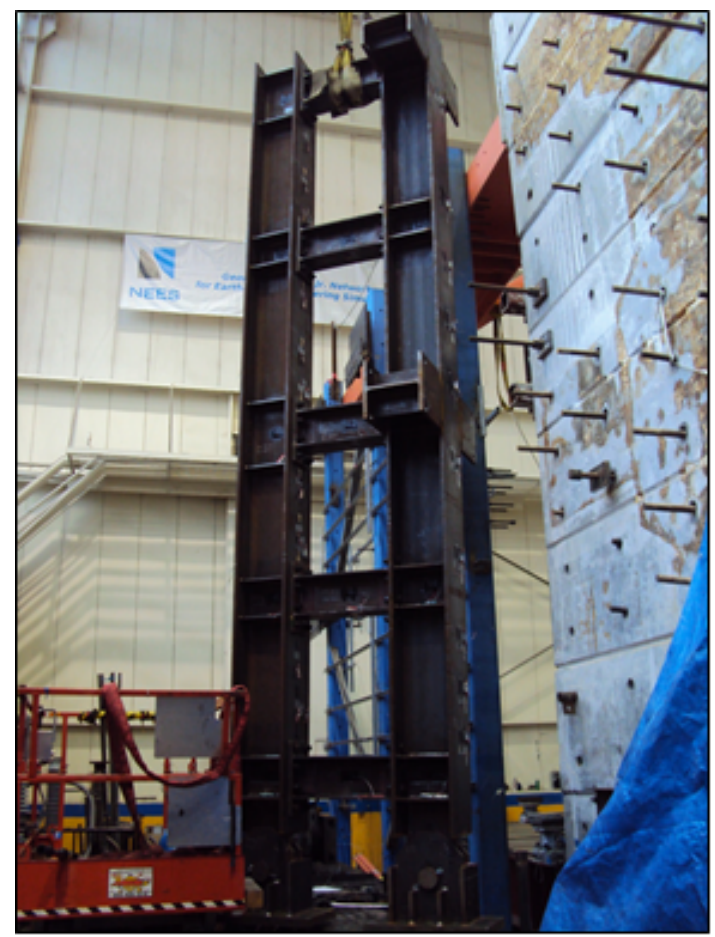

Figure 5.12: Linked columns of LCF-5L specimen being installed on the LC floor beam.

A foundation beam element, denoted hereafter as GC floor beam, was positioned on the strong floor in order to receive the gravity column. The gravity column base plate was attached to a load cell, which is in turn coupled to the GC floor beam, made of a W14x211 section, located at the top of the strong floor slab as shown in Figure (5.13). The 2" thick gravity column base plate was bolted to the load cell with 1" $\phi$ A490 bolts. The load cell was bolted to the GC floor beam with 7/8" $\phi$ A490 bolts through the top flange of the W14x211 section. The LC and the GC floor beams were connected to the strong floor by post-tensioned anchor rods.

After the gravity column was fully bolted to the GC floor beam, three sliding mechanisms per floor were positioned. Two of them (sliding mechanism A) were 

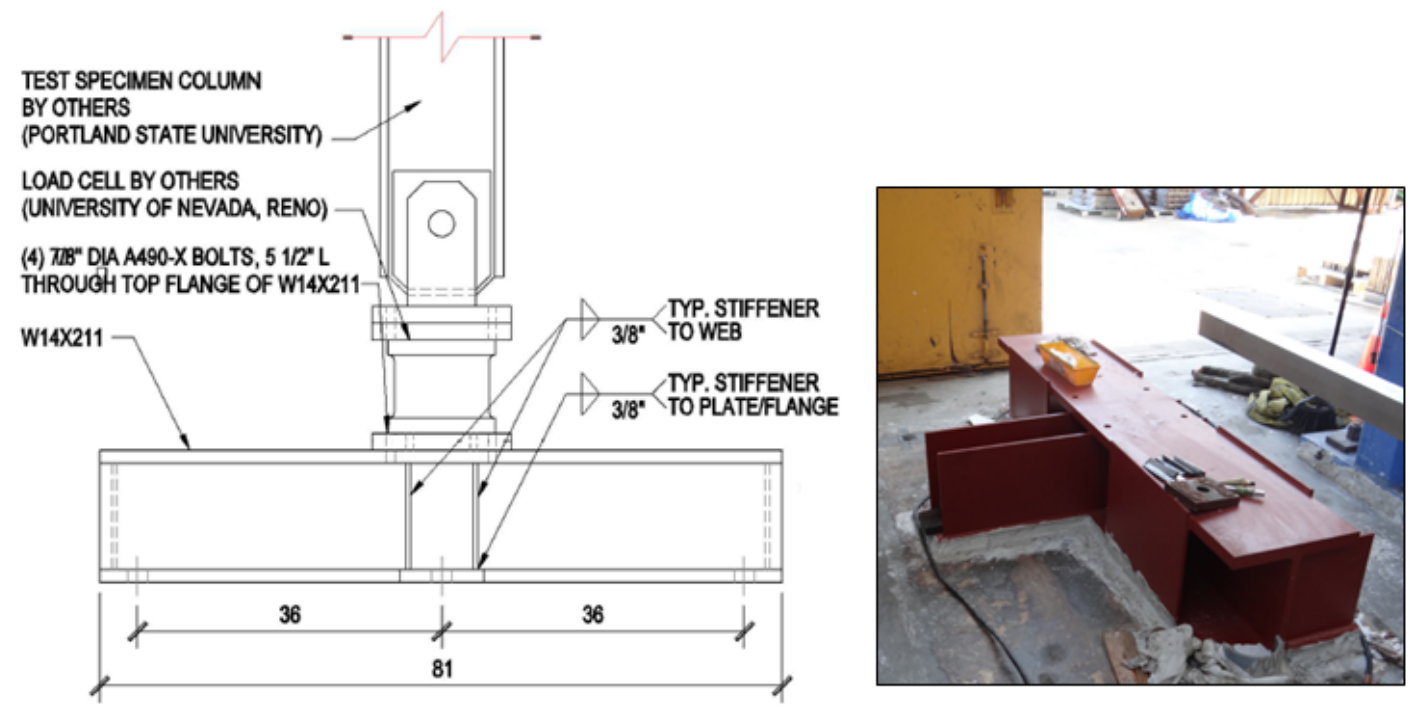

Figure 5.13: GC floor beam.

attached directly to the HSS columns while the other one (sliding mechanism B) was attached to the reaction wall. Sliding mechanism A already existed in the laboratory whereas sliding mechanism B had to be fabricated. The Tsection beam that is also part of the out-of-plane system, had to be fabricated, was then lifted and positioned in the sliding mechanisms. Figure (5.14) shows sliding mechanism B at the first floor level.

The first floor gravity beam was lifted and connected to the gravity column through a moment connection at one end and connected to the linked column through a shear connection at the other end. In the next step, the same procedure was done to connect the second floor gravity beam to the columns. Recall that gravity beam end-plate connections were shop welded using complete joint penetration at flanges and single pass fillet welds at webs. Column stiffeners and doubler plates were also shop welded to the columns using fillet welds. 


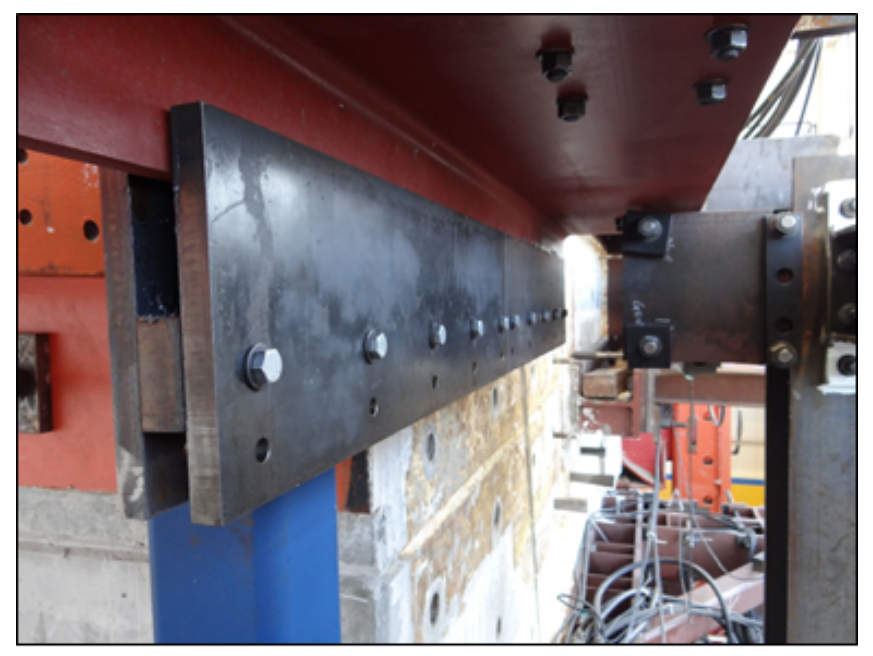

Figure 5.14: Sliding mechanism B at the first floor level.

After the second floor gravity beam was connected to the columns, a second T-section beam was then lifted and positioned in the sliding mechanisms. Each T-section was bolted to four continuity plates (PL 17x19x1/2") and these plates were in turn connected to the top flange of the gravity beam through fillet welds. Each T-section was also connected to the linked columns and to the gravity column via a set of plates bolted to the T-section and fillet welded to the stiffeners and flanges of the columns. Figure (5.15) shows the second floor T-section beam being lifted for installation. In regards to tightening the bolts, a torque-manual-wrench or a gun based wrench were used to tightened the fasteners and/or anchor bolts. They were tightened to the minimum required pretension forces specified either in the AISC manual or by the tool manufacturer. Actuators were then attached to the LCF system. The second specimen, LCF-3L, reused the same apparatus as the LCF-5L specimen, replacing only the damaged links. 


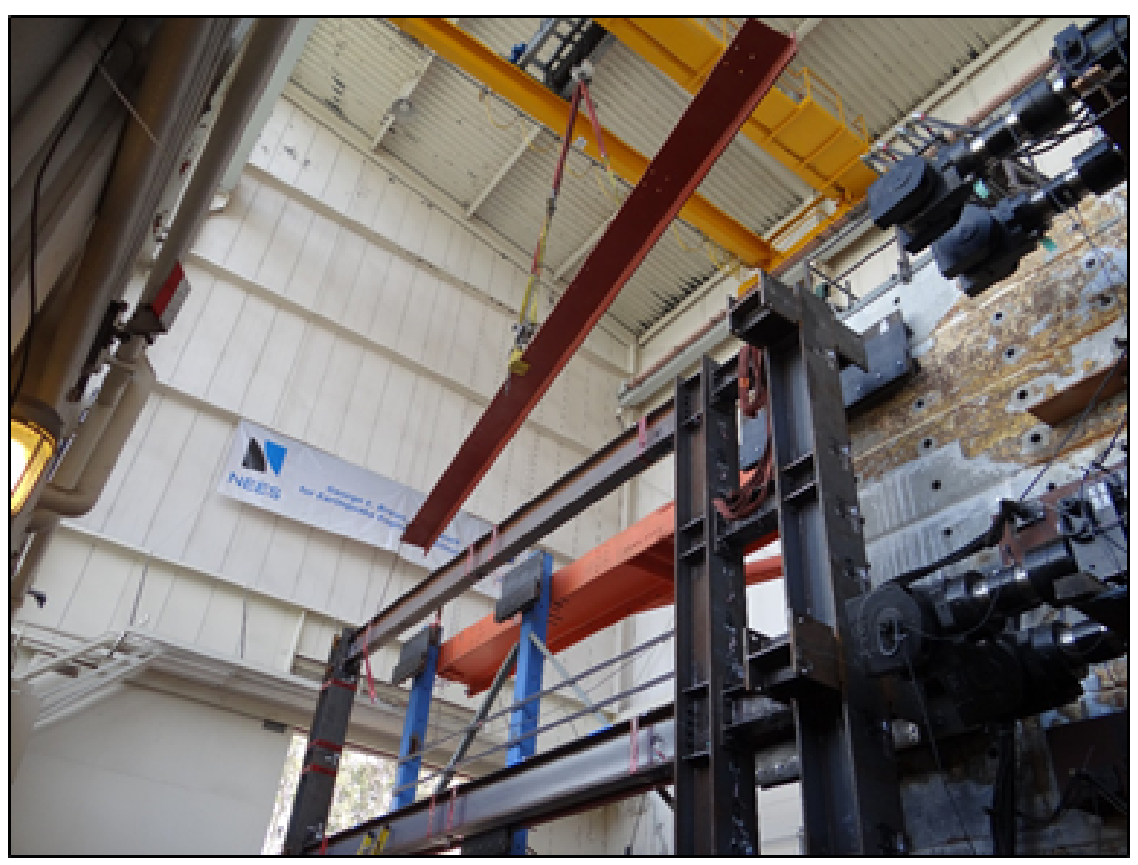

Figure 5.15: Second floor T-section beam being lifted.

\subsubsection{Actuators and Brackets}

Actuators for the experimental tests were selected from among those available in the laboratory, considering the loads and displacement targets. Thus, two static MTS 243.70T, 216 kips with \pm 72 inches stroke were used at the second story level and two dynamic MTS 244.51S, 220 kips with \pm 20 inches stroke were used at the first story level. The MTS 243.70T actuators have a hydraulically powered piston that can extend or retract (double-acting). The MTS 244.51S actuators were not only double-acting, but also double-ended where the actuator provides equal power in tension and compression. With these actuators and also considering the laboratory constraints, a second story level displacement equal to about $5 \%$ drift could be imposed, which corresponds to a displacement of 11 inches at the second floor level. The detailed drawings of the MTS 243.70T and 


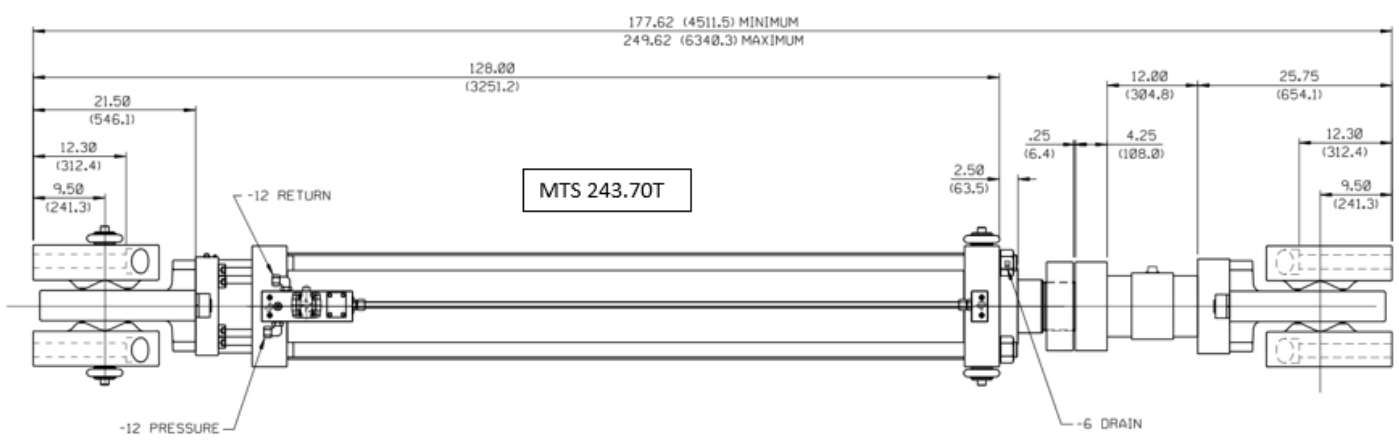

Figure 5.16: Static actuators used in the LCF system experimental test.

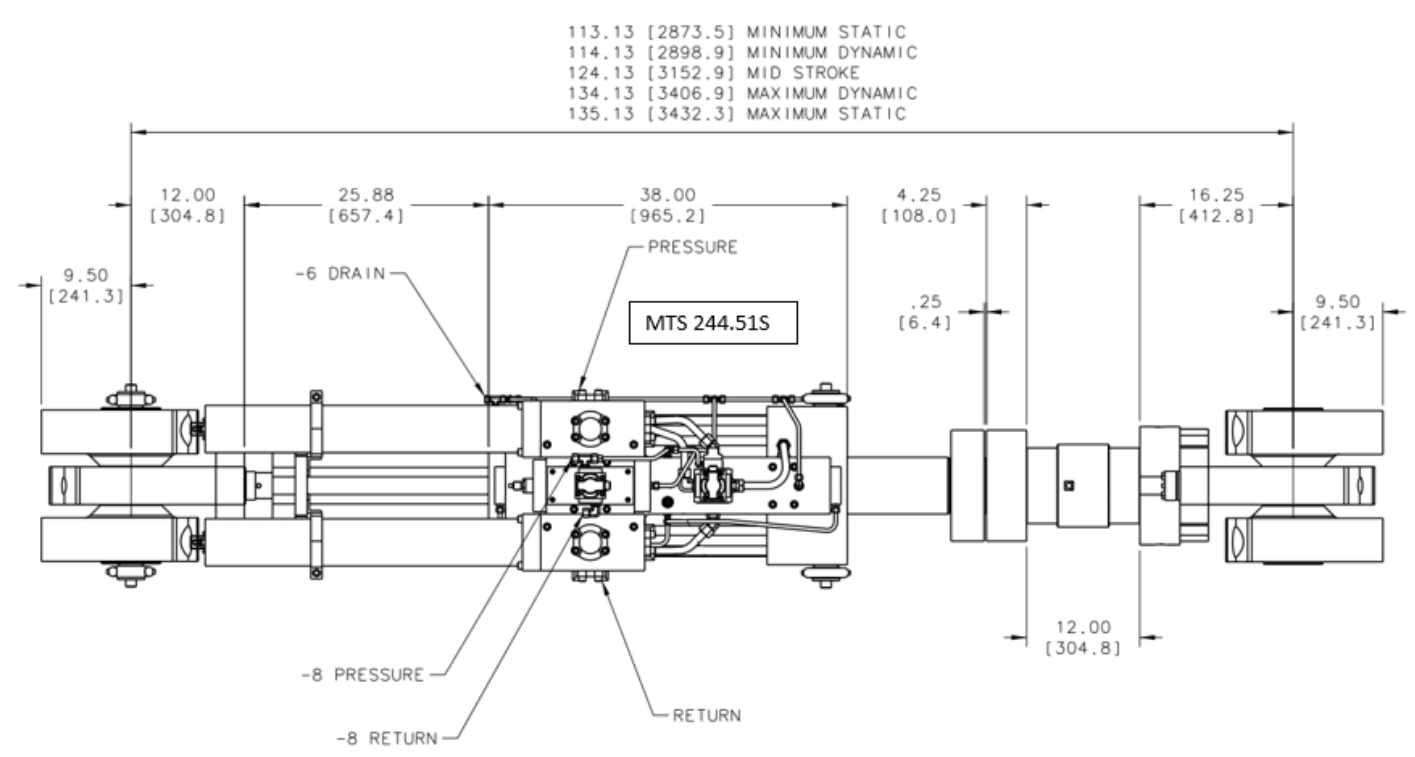

Figure 5.17: Dynamic actuators used in the LCF system experimental test.

MTS 244.51S actuators were provided by MTS and are shown in Figures (5.16) and (5.17), respectively.

Brackets on the reaction wall were needed in order to install the actuators. The upper bracket housed two static actuators whereas the lower bracket received the dynamic actuators. The capacity of each bracket is about 800 kips. Figure (5.18) shows the elevation view of the actuator brackets while Figure 


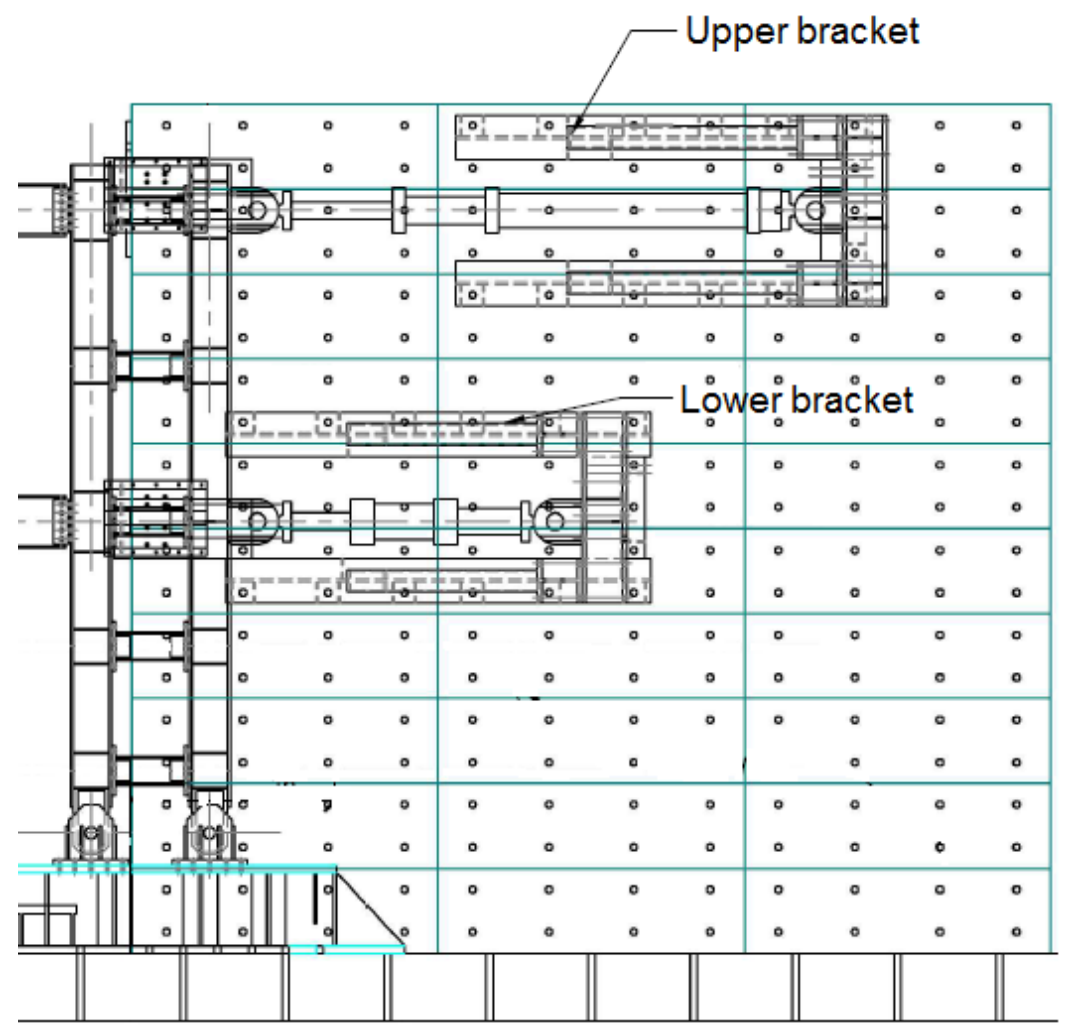

Figure 5.18: Elevation view of the actuator brackets.

(5.19) shows the actuator brackets installed on the reaction wall. Shop drawings for these brackets are presented in Appendix E.

\subsubsection{Actuator Spreader Beams}

In order to attach the actuators to the LCF system, two actuator spreader beams were designed. Each of the spreader beams consisted of two cantilever beams located at the floor levels to accommodate two actuators per floor. The cantilever beams were made of the same cross section and material as the linked columns and were designed to resist a bending moment of 4,950 kips.in and a shear of 220 kips. Stiffeners were designed accordingly. The cantilever beams 


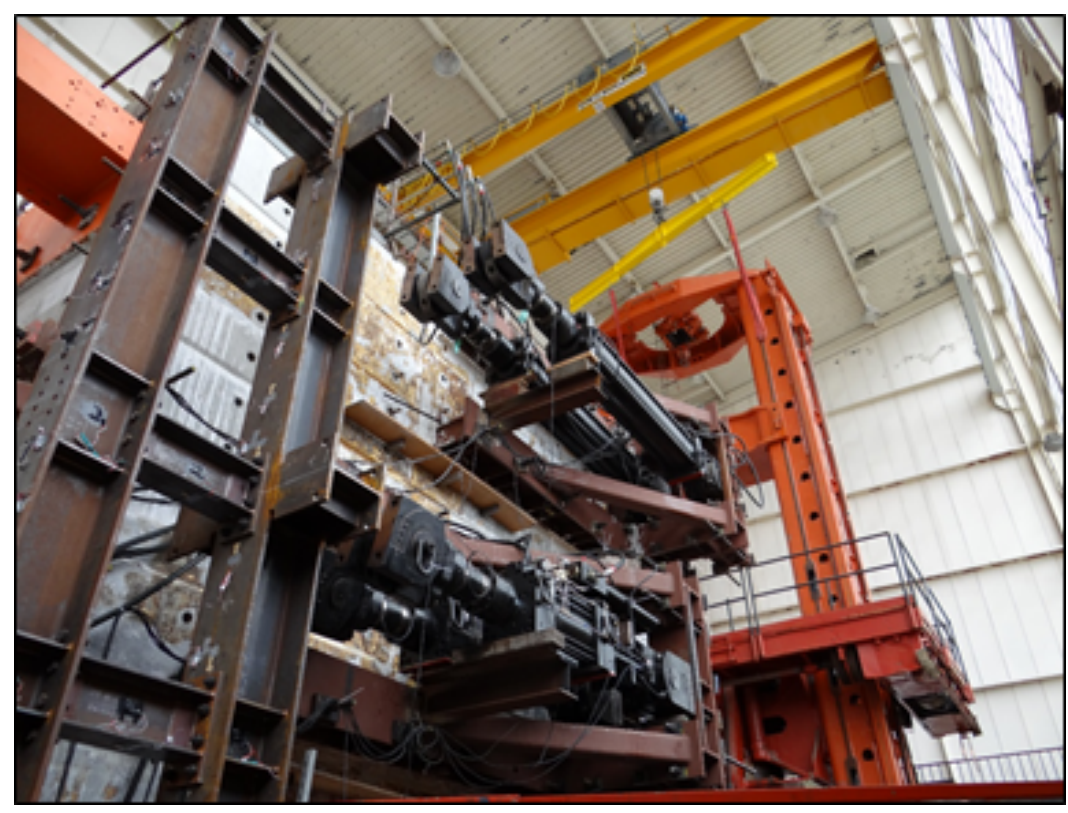

Figure 5.19: Actuator brackets installed on the reaction wall.

were shop welded to the linked column through a partial penetration bevel butt weld (both sides). Figure (5.20) shows the actuator spreader beam details while Figure (5.21) shows the actuator spreader beam located at the second floor before attaching the actuators.

\subsubsection{Out-of-Plane Restraint System}

The out-of-plane restraint system consisted of a cantilever beam fixed at the reaction wall, a T-section beam with three sliding mechanisms per floor, HSS columns, single plates, denoted hereafter connectors A (C-A), which connected the T-section to the gravity beam, a combination of plates and HSS sections, denoted hereafter connectors B (C-B), which connected the T-section to the gravity beams, and a set of plates, denoted hereafter connectors $\mathrm{C}(\mathrm{C}-\mathrm{C})$, which 


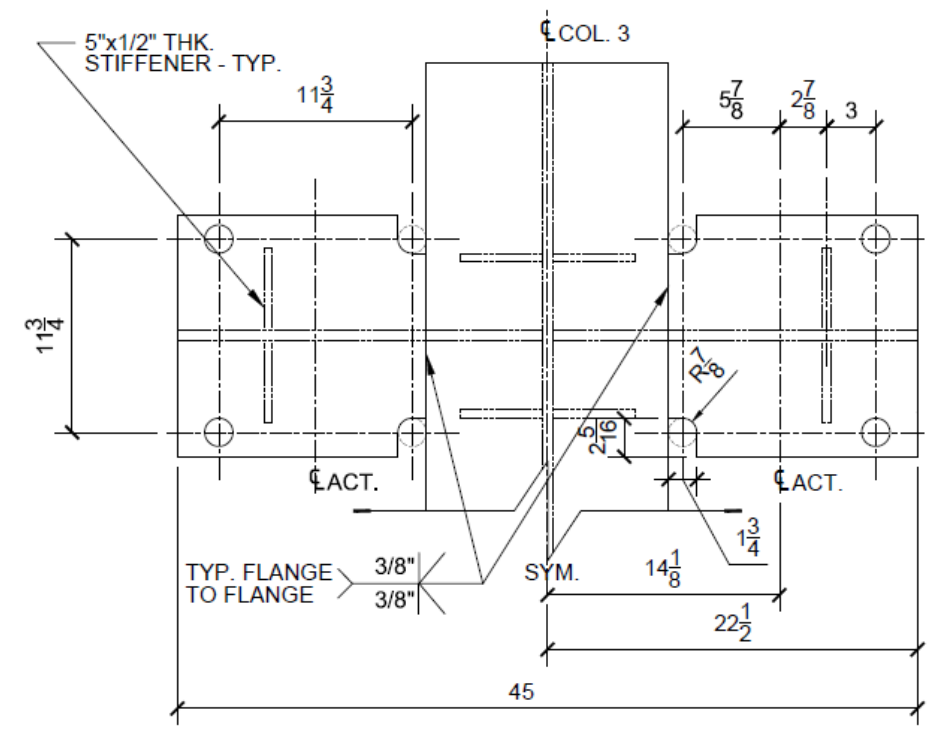

Figure 5.20: Actuator spreader beam details.

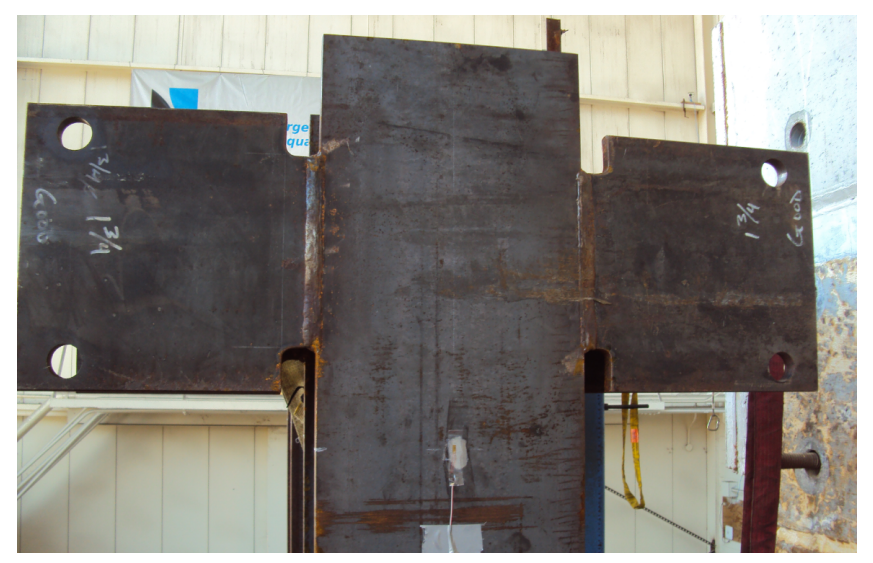

Figure 5.21: Actuator spreader beam located at the second floor. 
connected the T-section to the gravity and linked columns. There were three sliding mechanisms, two C-A, two C-B and three C-C connectors per floor. The T-section beam with $\mathrm{L}=31$ feet was made of a half W36x135 section. Figure (5.22) shows the highlighted out-of-plane restraint system used to avoid out-ofplane movement during testing.

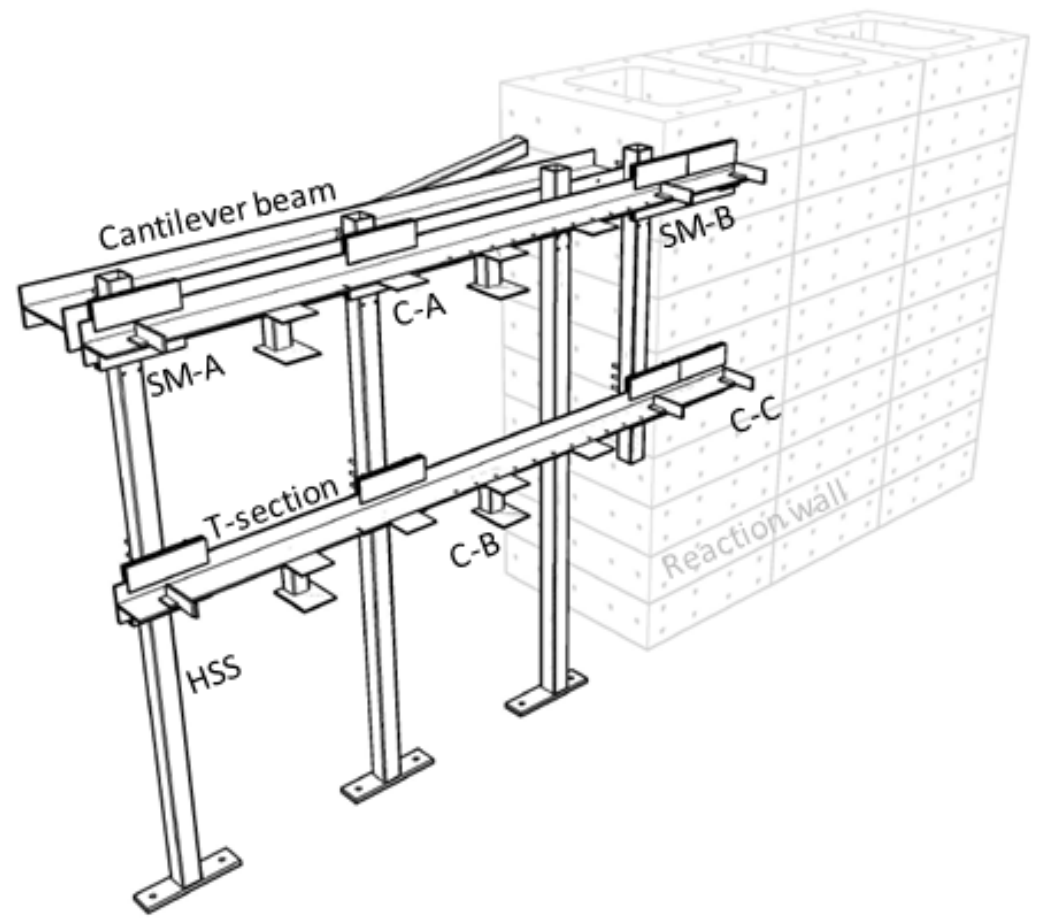

Figure 5.22: 3D view of the out-of-plane restraint system.

The connection between the T-section beam and the gravity beam was done via C-A connectors which consisted of a single plate (PL 17x19x1/2") bolted to the T-section beam and fillet welded to the top flange of the gravity beam. In order to avoid the movement of both flanges along the gravity beam, CB connectors were introduced. Figure (5.23) shows C-A and C-B connectors located at the second floor level. 


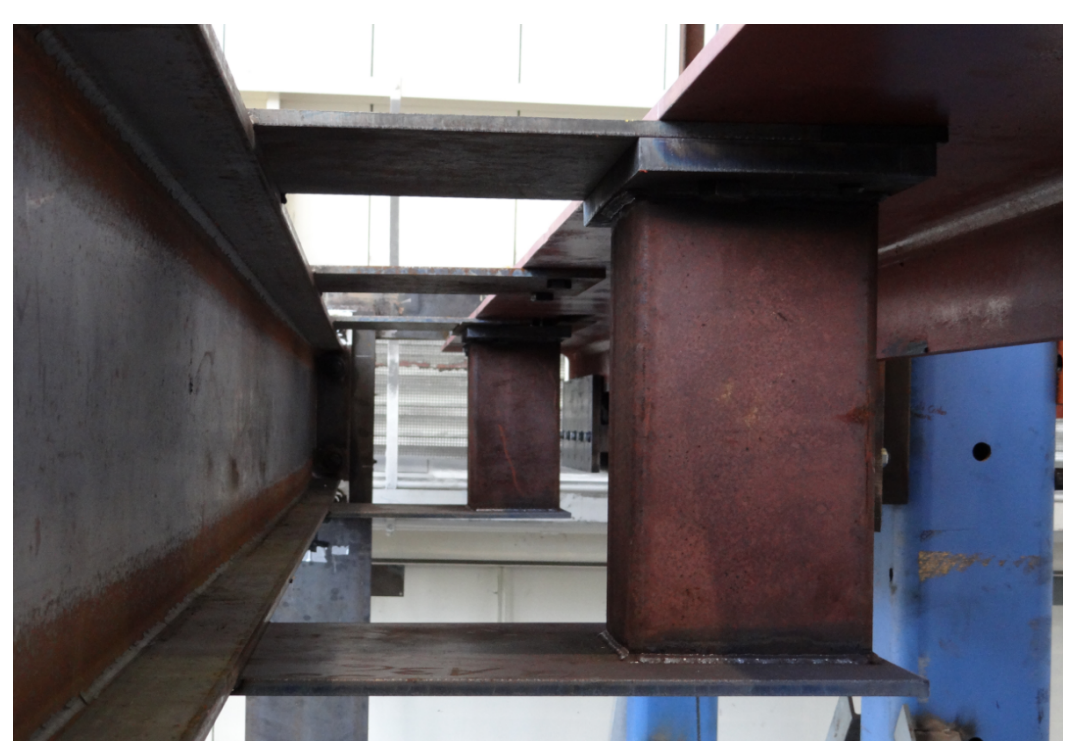

Figure 5.23: C-A and C-B connectors located at the second floor level.

C-B connectors consisted of a top plate (PL 17x19x1/2"), a small extension top plate (PL 17x3 3/4"x1/2"), a plate (PL 17x9x1") that was connected to the HSS, a bottom plate (PL 17x23x1/2"), and an HSS (8x8x1/4") section. The top plates were connected to the T-section and to the HSS plate using bolts. The connection between plate to HHS, HHS to bottom plate, bottom plate to the bottom flange of the gravity beam, and top plate to the top flange of the gravity beam were all done through fillet weld. Figure (5.24) shows the details of Connector C-B.

The role of Connector $\mathrm{C}-\mathrm{C}$ was to connect the T-section beam to either the gravity column or the linked columns. Connector C-C consisted of a vertical plate (PL 25 1/2"x8x1") and two horizontal plates (PL 11x4 1/2"x1/2"). Both horizontal plates were fillet welded to the vertical plate and also bolted to the T-section beam at one end. The other end connected the vertical plate to the 


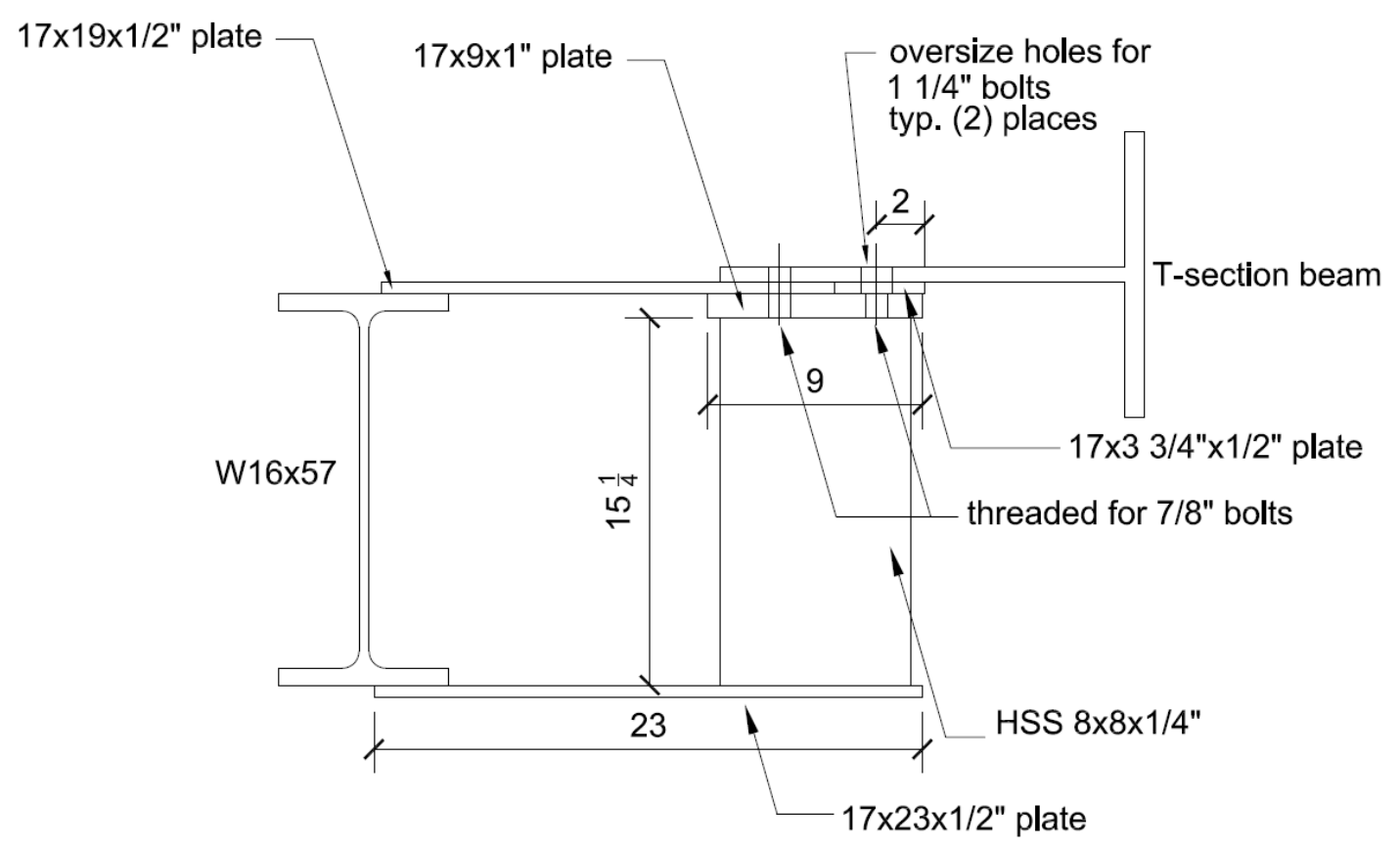

Figure 5.24: Connector C-B details.

column through fillet welds on both column stiffener and column web. Figure (5.25) shows the details of Connector C-C.

Sliding mechanisms A and B were responsible to receive the T-section beam. Sliding mechanism A consisted of a set of three types of plates: PL 36x11x1" (2x), PL36x26.5x1" and PL 36x3x1 3/8". A friction reducing grease was added between the sliding mechanism and the T-section beam to lubricate the steel plates. Figure (5.26) shows the sliding mechanism details as well as sliding mechanism A at first floor level. Sliding mechanism B was two times longer than sliding mechanism A to accommodate the T-section beam by the linked columns. The height of the plates was the same as sliding mechanism A and the plates were installed at the same elevation as well. The distance from the wall was controlled by adjusting nuts on all threaded rods. Four threaded rods 


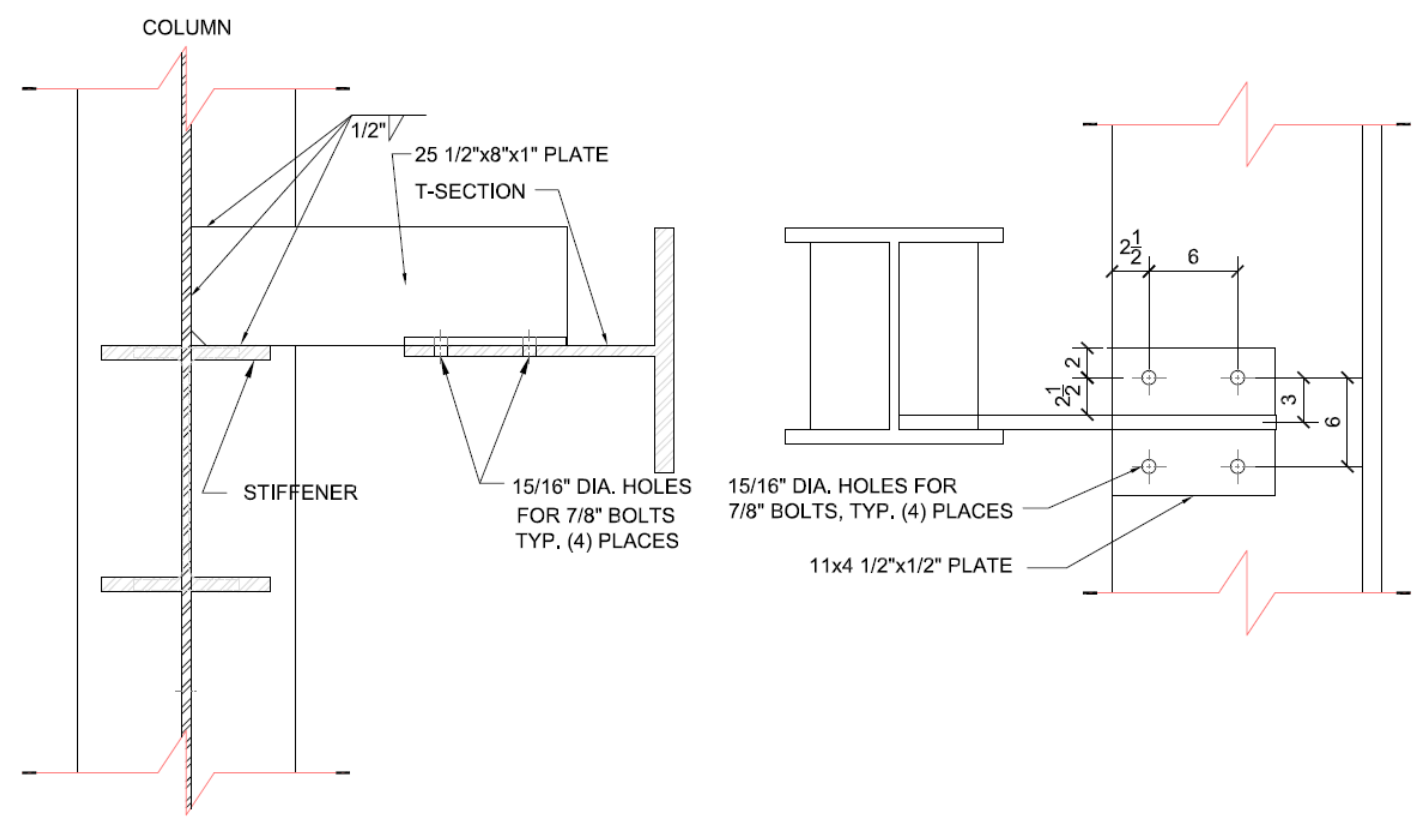

Figure 5.25: Connector C-C details.

with 1.5 in diameter connected the sliding mechanism B to the reaction wall. Two threaded rods went through the wall and two threaded rods went through the rib of the wall - per each elevation. The cantilevered part had two stiffeners attached to a base plate grouted and bolted to the east side of the wall. Figure (5.27) shows the sliding mechanism B located at the first floor level.

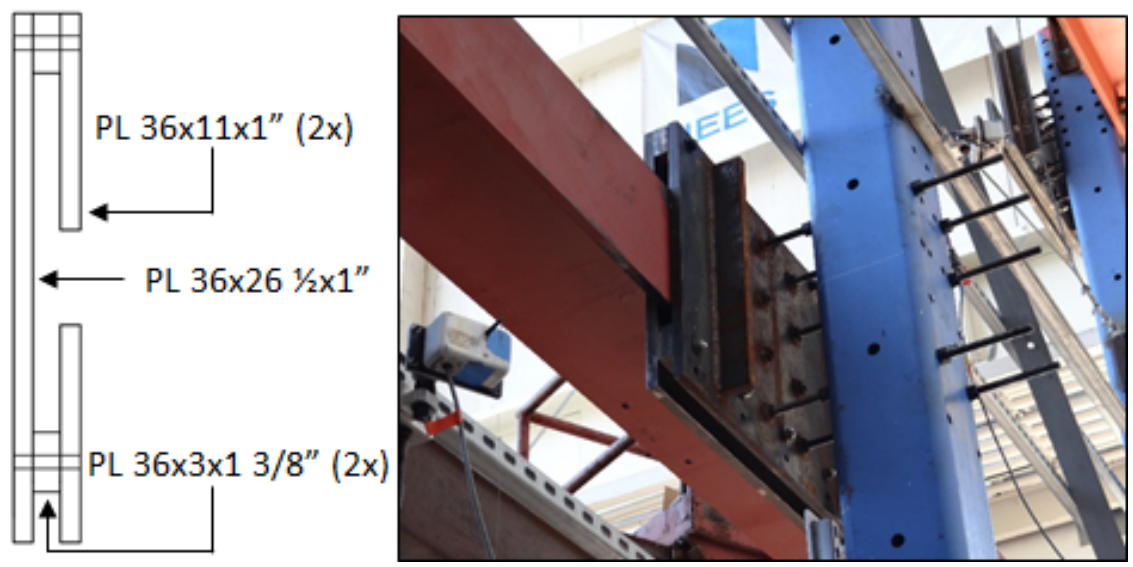

Figure 5.26: Sliding mechanism A at first floor level. 

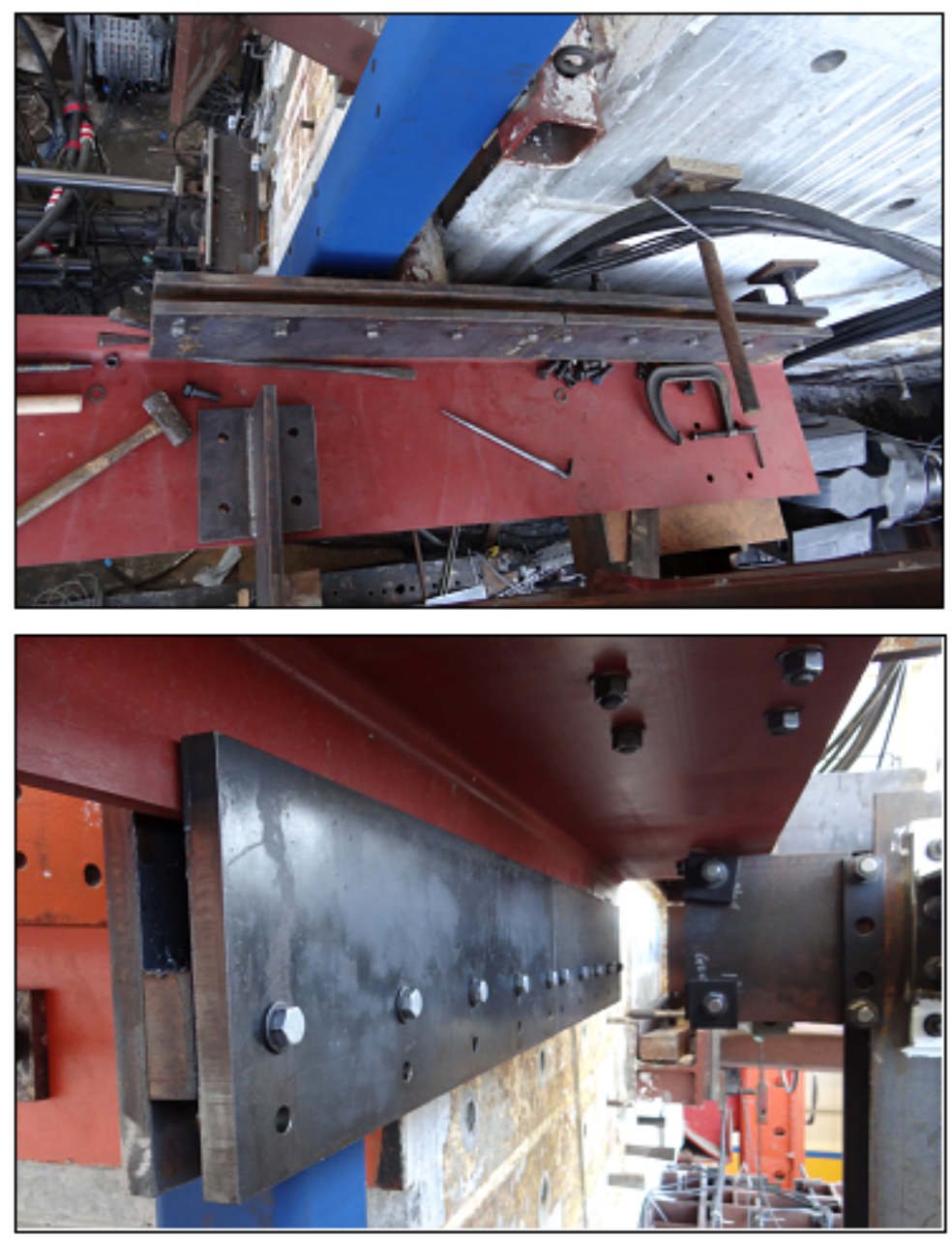

Figure 5.27: Sliding mechanism B at first floor level. 


\subsubsection{Completed Test Setup}

A test setup was devised for testing the LCF system, which consisted of LCF specimen, LC floor beam, GC floor beam, reaction wall, out-of-plane restraint system, and actuators. Parts of the specimen, such as locations where yielding was expected, were whitewashed with a lime and a water mixture intended to flake off as the steel yields, to illustrate the sequence and distribution of strain. During the many stages of the assemblage, the instrumentation was attached. Figure (5.28) shows the completed test setup for the LCF-5L specimen before testing.

\subsection{Instrumentation}

The response of the LCF steel system subjected to cyclic and earthquake loadings was measured using a total of 224 data acquisition channels. These channels collected data from strain gages, linear variable displacement transducers, load cells, string pots, accelerometers, and actuators using a National Instruments data acquisition system. Many of the measurements were used to calculate quantities such as column and link rotations, story drifts, average axial strains, bending moments, shears and axial loads. The detailed location of instrumentation points, channel numbers, cable numbers, and device types are listed in Appendix F. 


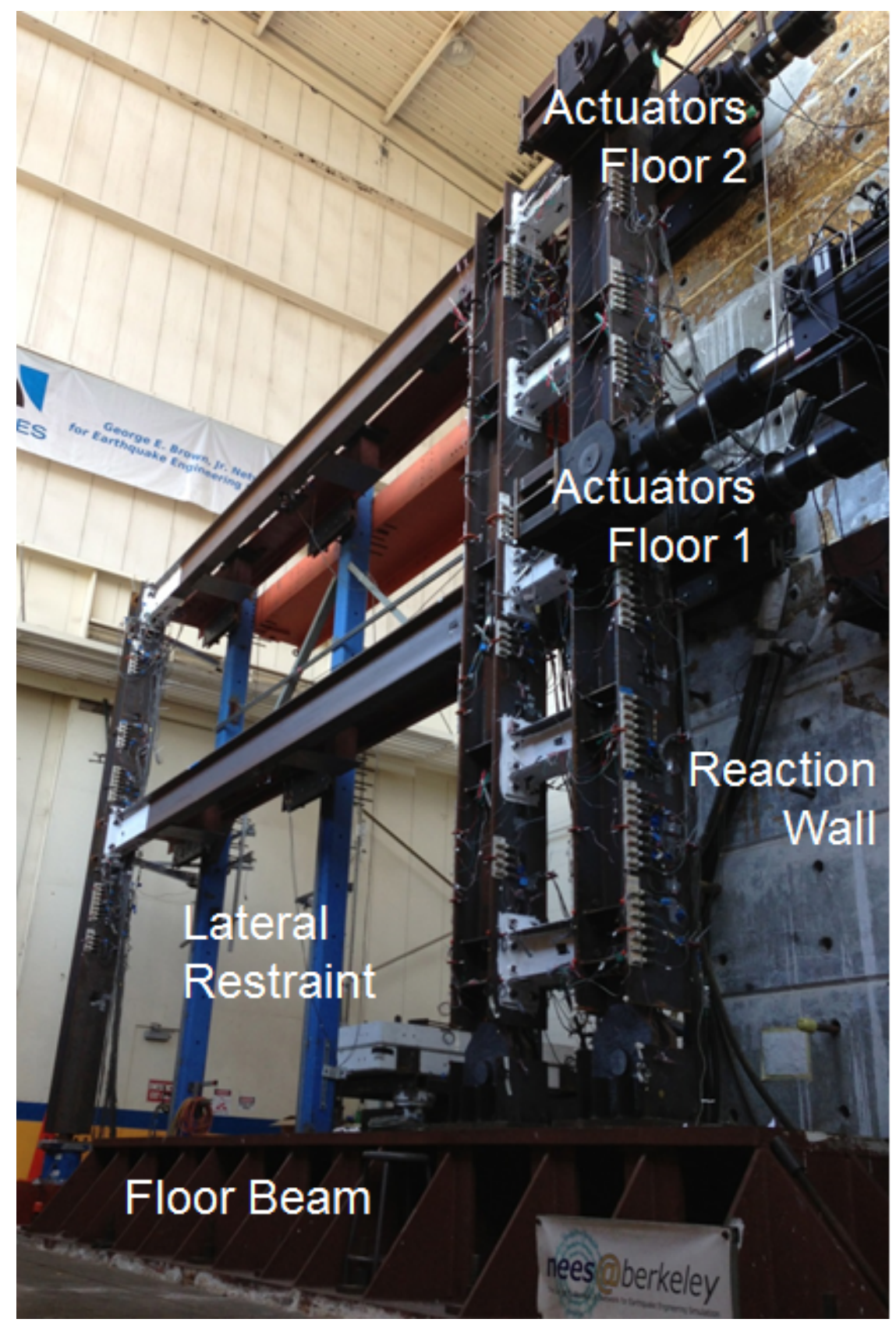

Figure 5.28: Completed test setup for the LCF-5L specimen before testing. 


\subsubsection{Strain gages}

Four types of strain gages were used, namely, general purpose uniaxial gage, large deformation uniaxial gage, post yield uniaxial gage and rosettes. General purpose uniaxial gage was manufactured by Vishay Measurements Group and others by Tokyo Sokki Kenkyujo. The general purpose uniaxial gage were type C2A-06-250LW-120, denoted hereafter as C2A, and were placed along column flanges in both linked and gravity columns. They were also placed on top and bottom flanges of the GBs at mid-section and near the shear connection, totalizing six gages per GB. Additionally, ten $\mathrm{C} 2 \mathrm{~A}$ gages were placed along the GC flanges in order to estimate the axial load and to determine if yielding had occurred. The same approach was used in LC-1 and LC-2 with twenty six and twenty two gages used, respectively. This was done to understand the strain distribution and variation across the column flanges.

The large deformation uniaxial gages were type YEFLA-5-2L-120, denoted hereafter as YEFLA, and were placed along flanges of the shear links. Three gages were attached on top and bottom flanges of the links and two gages were attached on top and bottom flanges of the GBs close to the moment connection. The post yield uniaxial gages were type YFLA-5-2L-120, denoted hereafter as YFLA, with three gages attached to the top and bottom flanges of the GBs close to the moment connection. Finally, rosettes were type YEFRA-5-2L-120, denoted hereafter as rosette, and were placed on the link web and column web 


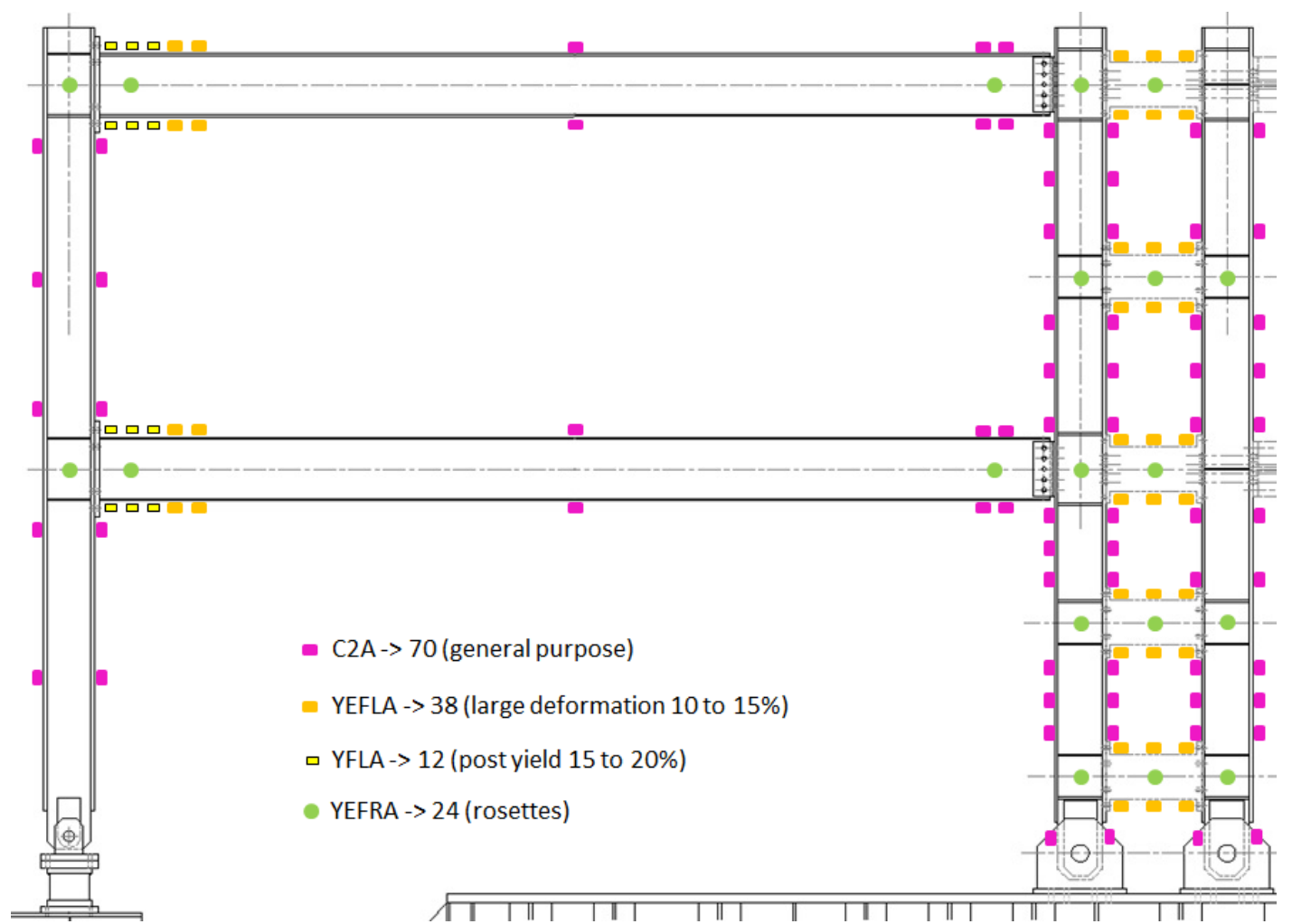

Figure 5.29: Strain gage layout used on LCF test assembly.

(panel zone). Their locations were the following: one rosette per link at link web, eight rosettes on LCs web between column stiffeners, two rosettes on GC between column stiffeners, and two rosettes per GB one at each end. For LCF5L specimen, a total of 139 strain gages were used as follows: 70 C2A, 38 YEFLA, 12 YFLA, and 19 rosettes. For LCF-3L, the same configuration for GC, LCs, GBs were used and only three links were instrumented. Figure (5.29) shows the strain gage layout used on LCF-5L specimen assembly.

Before the installation of the strain gages, a rust layer was removed from the steel surface using a $120^{\circ}$ Angle Air Die Grinder to make sure the strain gages were properly attached to the base metal through a M-Bond 200 adhesive. The 

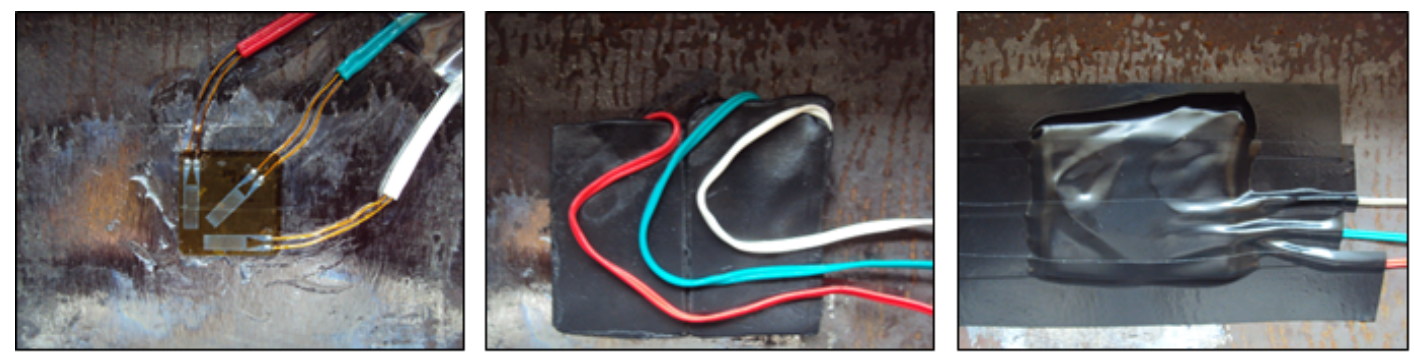

Figure 5.30: Strain gage protection.

installation itself followed the procedure given by the manufacturer. All strain gages were protected with an air-drying polyurethane coating and also with a butyl rubber coating followed by an electrical tape cover as shown in Figure $(5.30)$.

\subsubsection{LVDTs}

Linear Variable Displacement Transducers (LVDTs) were used to measure the column panel zone rotations, link and column rotations, connection slippage, and base plate slippage. The LVDTs used were Novotechnik TLH and TR models with ranges between 1 and 20 inches. The holders for these LVDTs were either tack-welded or C-clamped to the specimen. All LVDTs were calibrated using Mitutoyo gage blocks (0.2 in, 0.3 in, 0.5 in, 1.0 in, 2.0 in and 5.0 in).

Figure (5.31) shows the LVDT gage layout used on LCF-5L specimen assembly. 


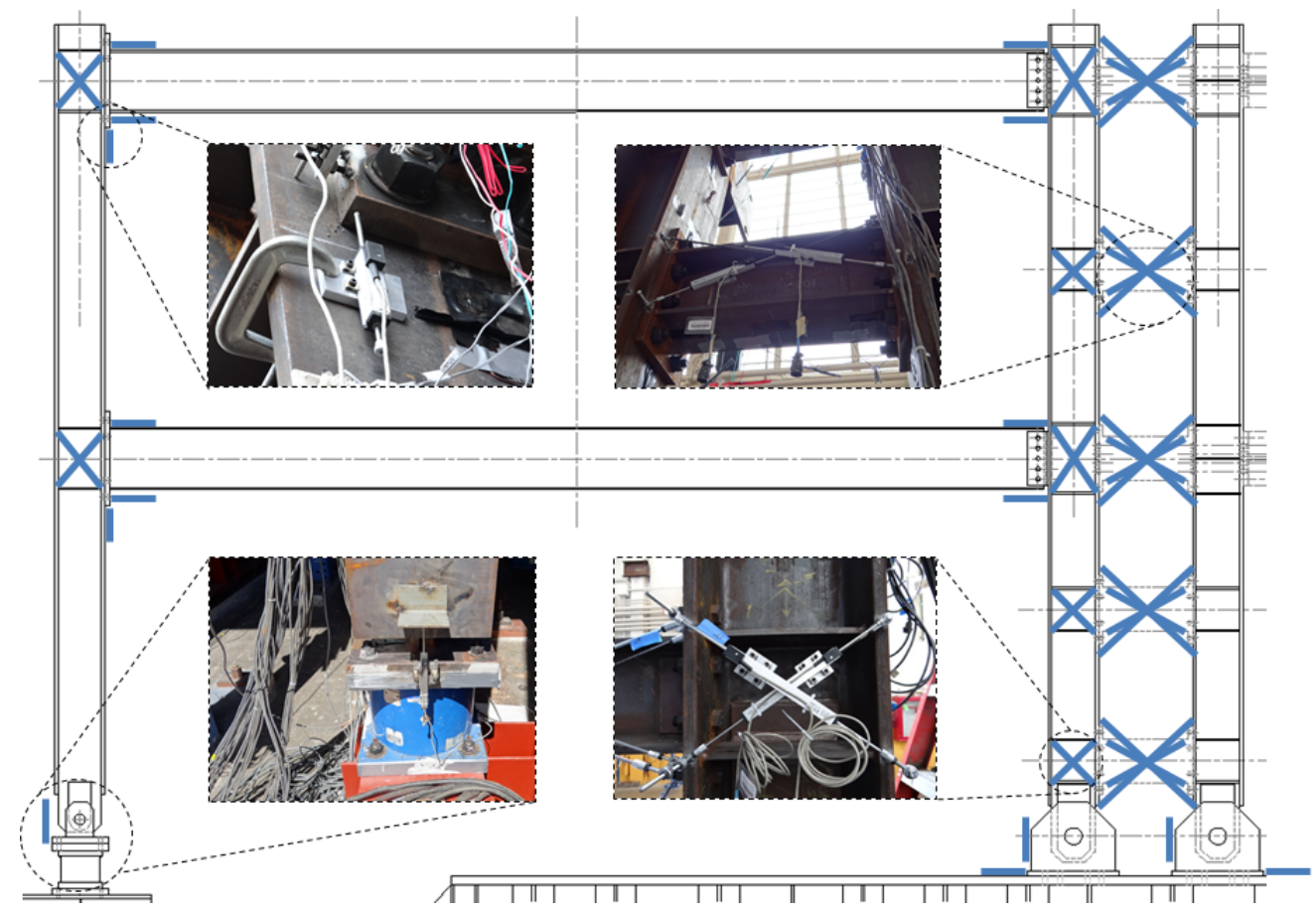

Figure 5.31: LVDT layout used on LCF test assembly.

\subsubsection{Load Cells}

The loads transfered to the specimen were measured with load cells within the actuators while the loads transferred to the gravity column were measured with a 6 degrees of freedom load cell provided by University of Nevada at Reno. Reno's load cell was positioned between the GC strong floor attached to the strong floor and the gravity column and had the following capacity: 200 kips for axial, 200 kips for shear, 500 kips-in for moment, and 700 kips-in for torque. Figure (5.32) shows the load cell already installed on the gravity column and also the load cell cross-section. 

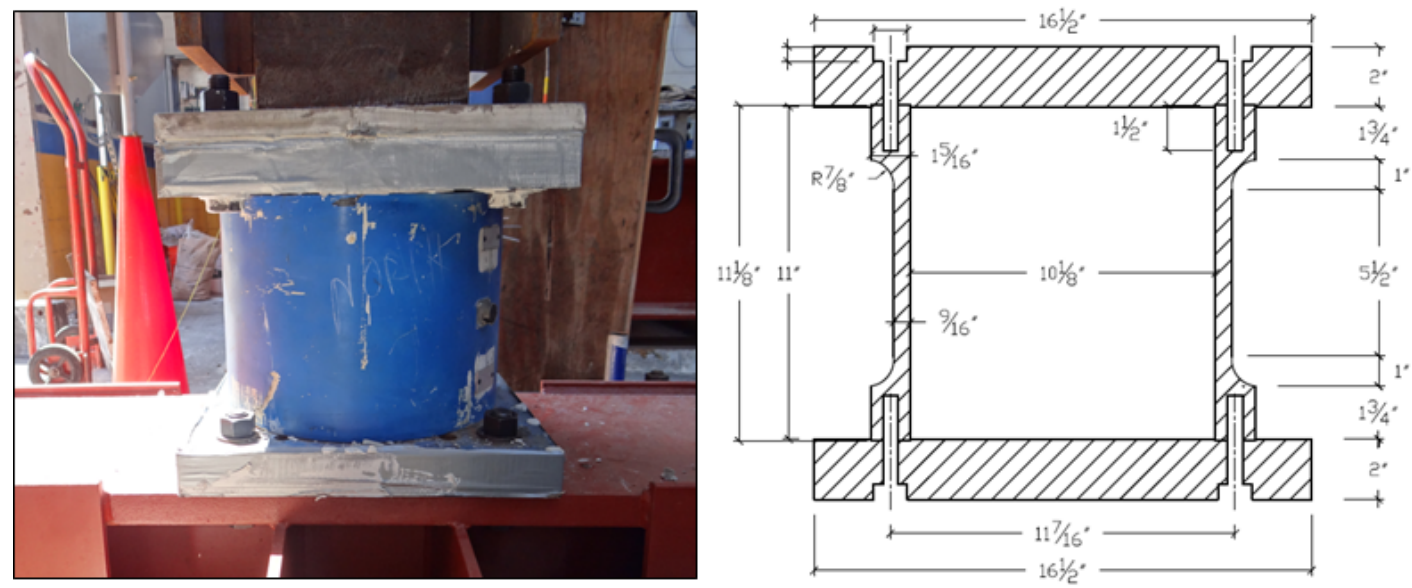

Figure 5.32: Load cell already installed and load cell cross-section.

\subsubsection{String Pots and Accelerometers}

First and second stories were also instrumented with string pots to measure story displacements. They were mounted directly to the gravity column at each floor level. The string pots used were Celesco PT 101 models with total movement ranges between 2 and 30 inches. Figure (5.33) shows a string pot at the first story level.
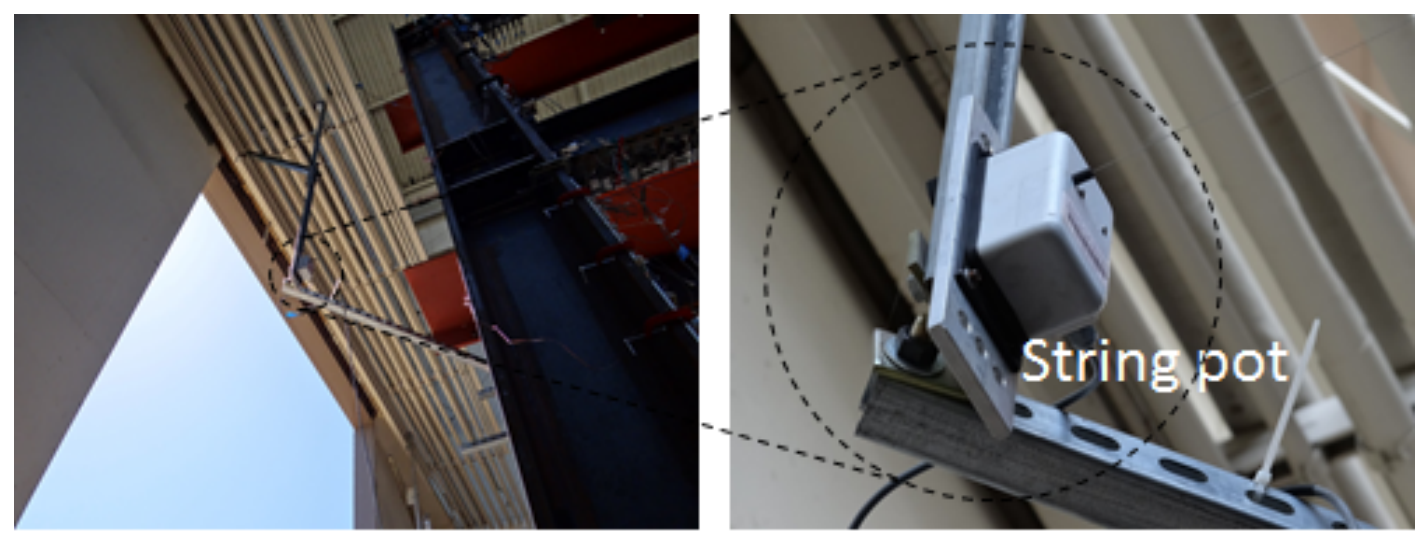

Figure 5.33: String pot at the first story level. 


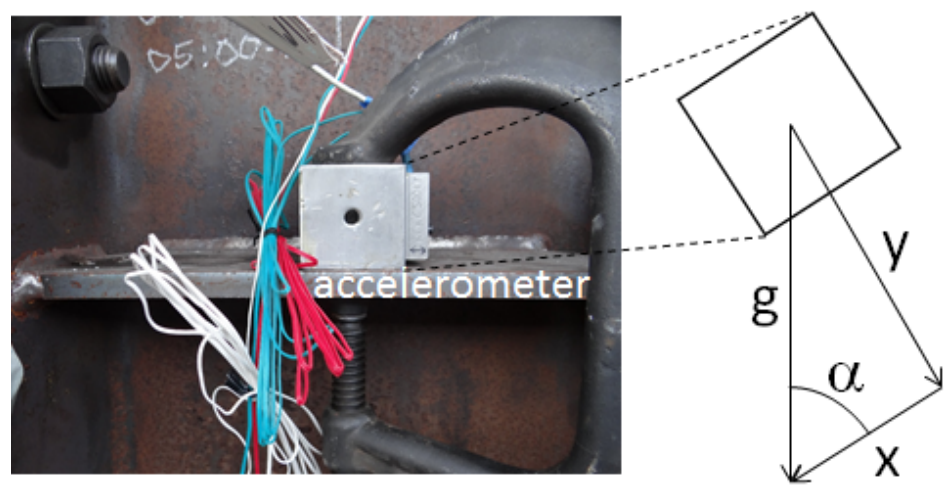

Figure 5.34: Accelerometer used to obtain rotation.

To capture the rotation of some joints, tiltmeters were attempted to be used. However, due to calibration issues the tiltmeters were disregarded. Six in-house three-axes accelerometers were used instead. The gravitational acceleration always acts perpendicular to the earth's surface. So when the LCF system is tilted at an angle $\alpha$, part of that acceleration acts along $x$ and $y$ axes. Hence, the rotation was calculated using trigonometry. Figure (5.34) shows one of the accelerometers used and also a sketch to obtain the rotation. Accelerometers were attached to both linked and gravity columns stiffeners as shown in Figure (5.35). String pots and accelerometers layout used on LCF test assembly is also shown in Figure (5.35).

\subsubsection{Cameras and Video}

Digital photos in different views and angles were taken and stored in a desktop computer. Five digital single-lens reflex cameras were connected to desktop computers and shot the still photos every 10 seconds continuously throughout the 


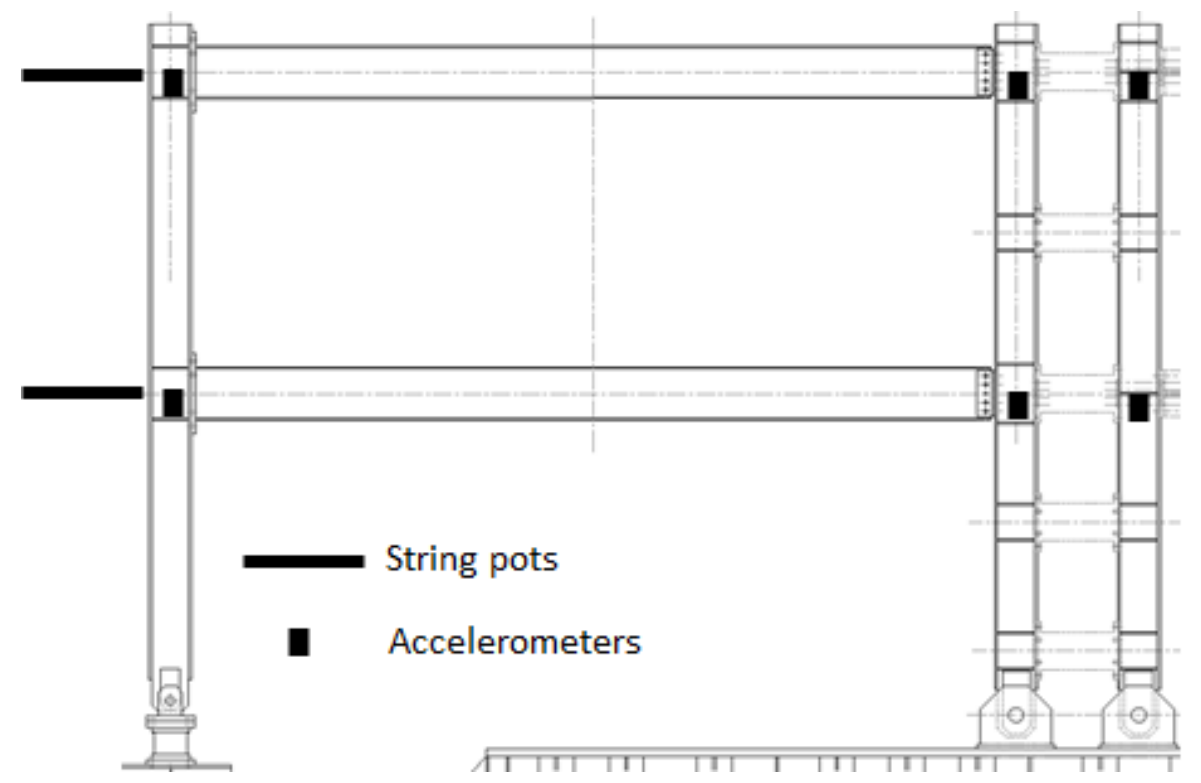

Figure 5.35: String pots and accelerometers layout used on LCF test assembly.

entire test. Digital videos of each test were also recorded. A three-dimensional Leica high definition laser scanner was also used to capture the specimen deformed shape throughout the tests. Unfortunately, the laser scanner did not work as expected. Figure (5.36) shows cameras and laser scanner used on LCF test assembly.

\subsubsection{Data Acquisition System}

All test data were measured using a high performance transducer signal conditioning and system control named 6000DAS. Data were displayed and recorded using Simulink. Instruments were connected to switch boxes using cable extension as shown in Figure (5.37). These switch boxes were integrated into a high-speed data acquisition system programmed to scan every channel and 


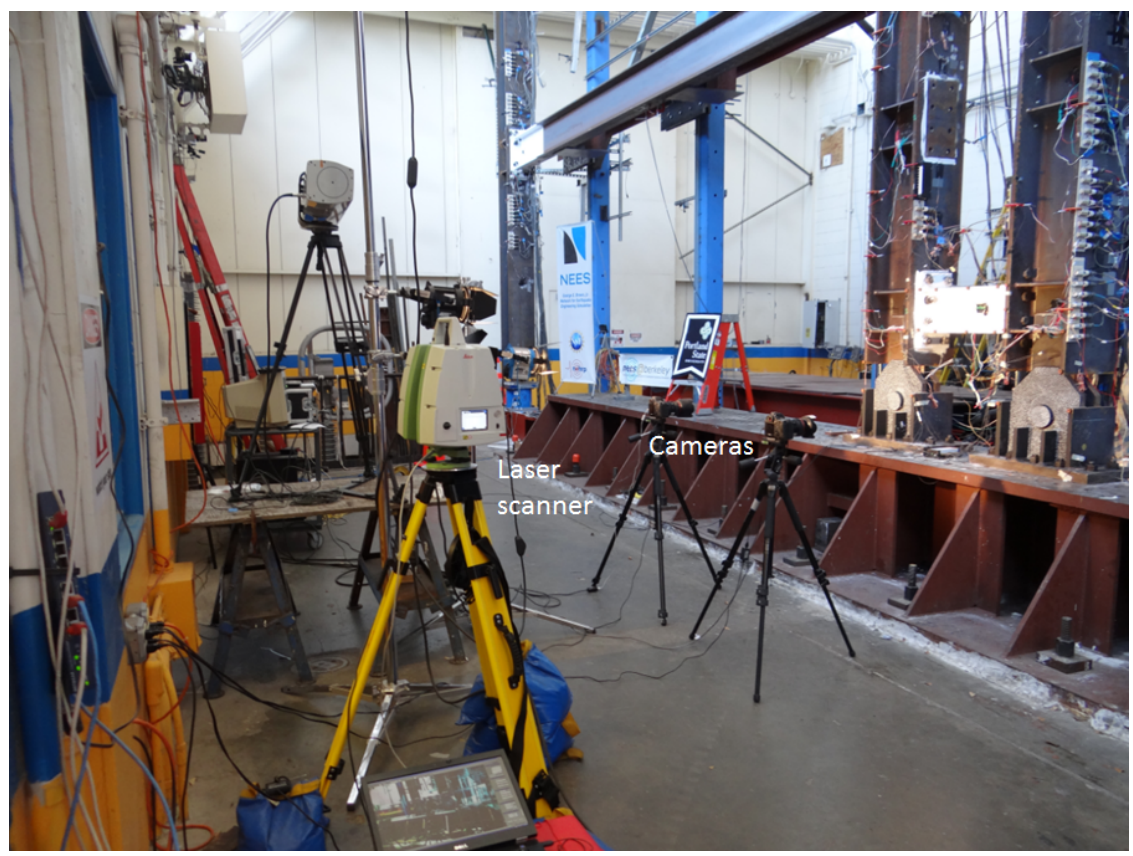

Figure 5.36: Cameras and laser scanner used on LCF test assembly.

record the data into a desktop computer under specified sampling rate defined before testing.

All instruments were attached to cables in one end while the other end was connected to the switch boxes. To expedite the process cables were grouped in bundles. Strain gages were also connected to an intermediate device attached directly to the LCF specimen to facilitate the connection to the switch boxes as shown in Figure (5.38).

\subsection{Cyclic Loading Protocol}

Two LCF systems were tested following the loading protocol described below. One of three loading protocols were to be used during the experimental tests: 

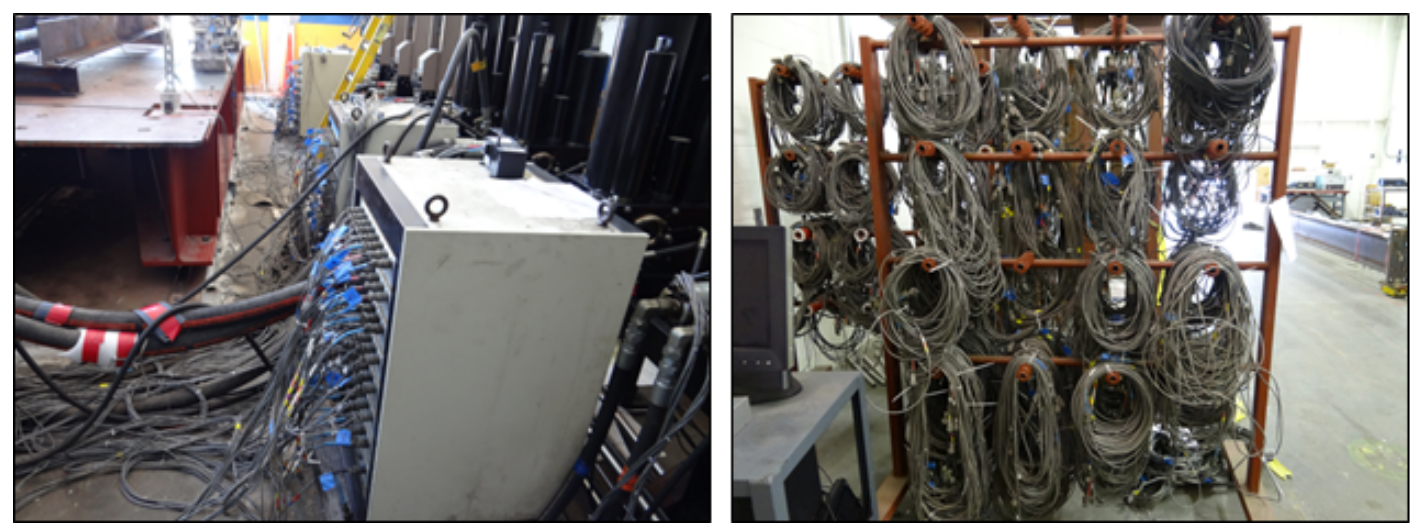

Figure 5.37: Switch boxes and cable extension.

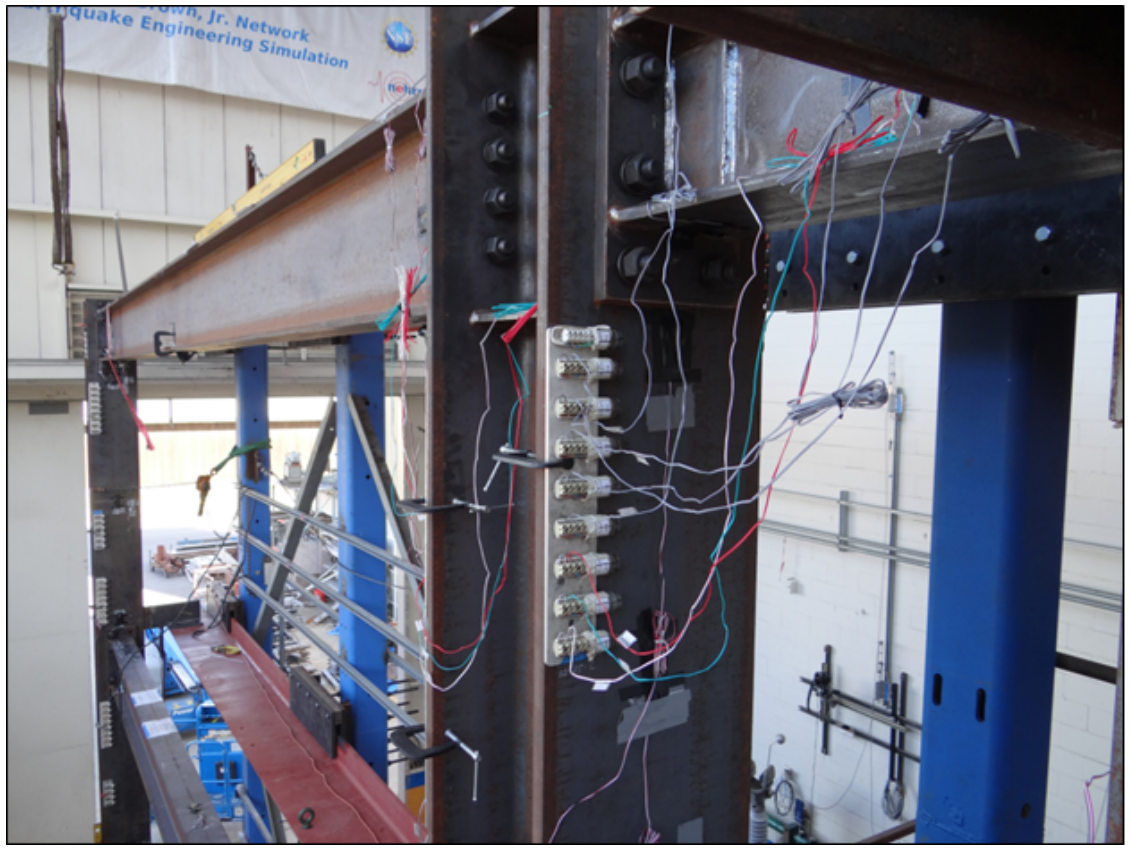

Figure 5.38: Intermediate gage device attached to the LCF specimen. 
SAC, ATC-24 or FEMA 461. For steel frame structures the story yield drift is expected to happen around $0.01 \mathrm{rad}$., which permits an approximate correlation between SAC and ATC-24 protocols. However, during the pre-yield cyclic test, on the LCF-5L specimen, the story yield drift was lot smaller. Therefore, the FEMA 461 protocol was the chosen one and this protocol is also applicable to drift sensitive structural components. FEMA 461 has a protocol named "QuasiStatic Testing on Structural and Non-Structural Components and Systems" and should be used for the determination of performance characteristics of components (i.e. frame assemblies), the behavior of which is primarily controlled by the application of seismic forces or seismic-induced displacements.

FEMA 461 protocol calls for two targets $\left(\Delta_{0}\right.$ and $\left.\Delta_{m}\right)$ and a predetermined number of increments (n), where $\Delta_{0}$ is the targeted smallest deformation amplitude of the loading history, $\Delta_{m}$ is the targeted maximum deformation amplitude of the loading history and $n$ is the number of steps or increments in the loading history. For testing purposes, Table (5.1) summarizes the parameters used on both LCF specimens while Figure (5.39) shows the loading history used on LCF test assembly up to $0.05 \mathrm{rad}$. story drift. The testing was conducted in a slow, displacement-controlled, and predetermined manner.

Table 5.1: FEMA 461 parameters.

\begin{tabular}{ccccc}
\hline Specimen & $\mathrm{n}$ & $\Delta_{0}(\mathrm{rad})$ & $\Delta_{m}(\mathrm{rad})$ & Drift $(\%)$ \\
\hline LCF-5L & 40 & 0.0012 & 0.026 & 2.6 \\
LCF-3L & 51 & 0.0012 & 0.050 & 5.0 \\
\hline
\end{tabular}




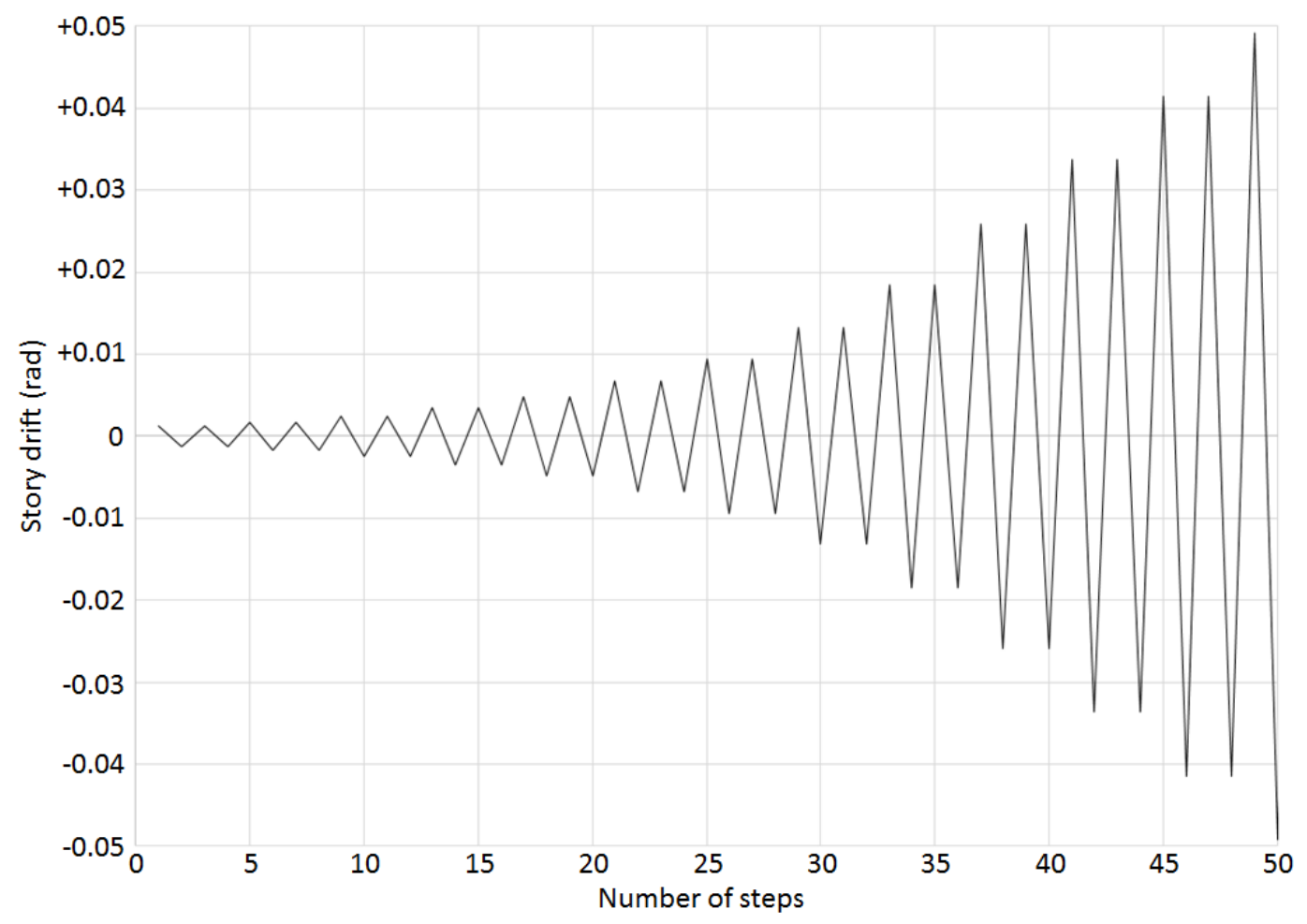

Figure 5.39: Displacement-controlled loading history used on LCF test assembly.

Since the experimental behavior of the LCF system was unknown, a quasistatic testing up to $2.6 \%$ drift was conducted first on the LCF-5L sub-system, followed by a sequence of hybrid testing on the entire system. For the LCF3L specimen, hybrid testing was conducted first and then the physical LCF-3L sub-system was tested under an ultimate cyclic loading up to $5 \%$ total drift. Hybrid simulation is discussed in Section (5.6).

\subsection{Hybrid Simulation}

Over the last years, the seismic behavior of steel structures has been the subject of extended research and several experimental tests on such structures were 
conducted. In most cases, these structures were either subjected to quasi-static cyclic loading in which the dynamic response of the system cannot be captured or were tested on a shaking table. Typically, it is very difficult to do a large or full scale test using a shake table due to limitations on table capacity. Seismic tests may also involve hybrid simulation, a combination of physical test of a critical substructure tied to a numerical model of the building. In a hybrid simulation test, the well understood part of the structure is modeled in a finite element program and the critical substructure is built in the laboratory to be tested.

Hybrid simulation offers an efficient method for assessment of dynamic behavior of large-scale structural systems subjected to earthquake excitation. Compared to earthquake simulations using shake tables, hybrid simulation may have significant advantages in terms of cost, geometry, and required physical mass of structures and components that can be tested. By this token, a LCF system was investigated experimentally to validate the rapid return to occupancy performance at a system level. The hybrid simulation test setup used on LCF5L test assembly is illustrated in Figure (5.40). P-delta effects were included on LCF-3L test assembly and results are discussed in Chapter (6). Ground motion intensity was selected such that three distinct performance levels were induced in the LCF specimen: linearly elastic, rapid return to occupancy corresponding to a moderate damage state, and collapse prevention corresponding to a significant damage state. 


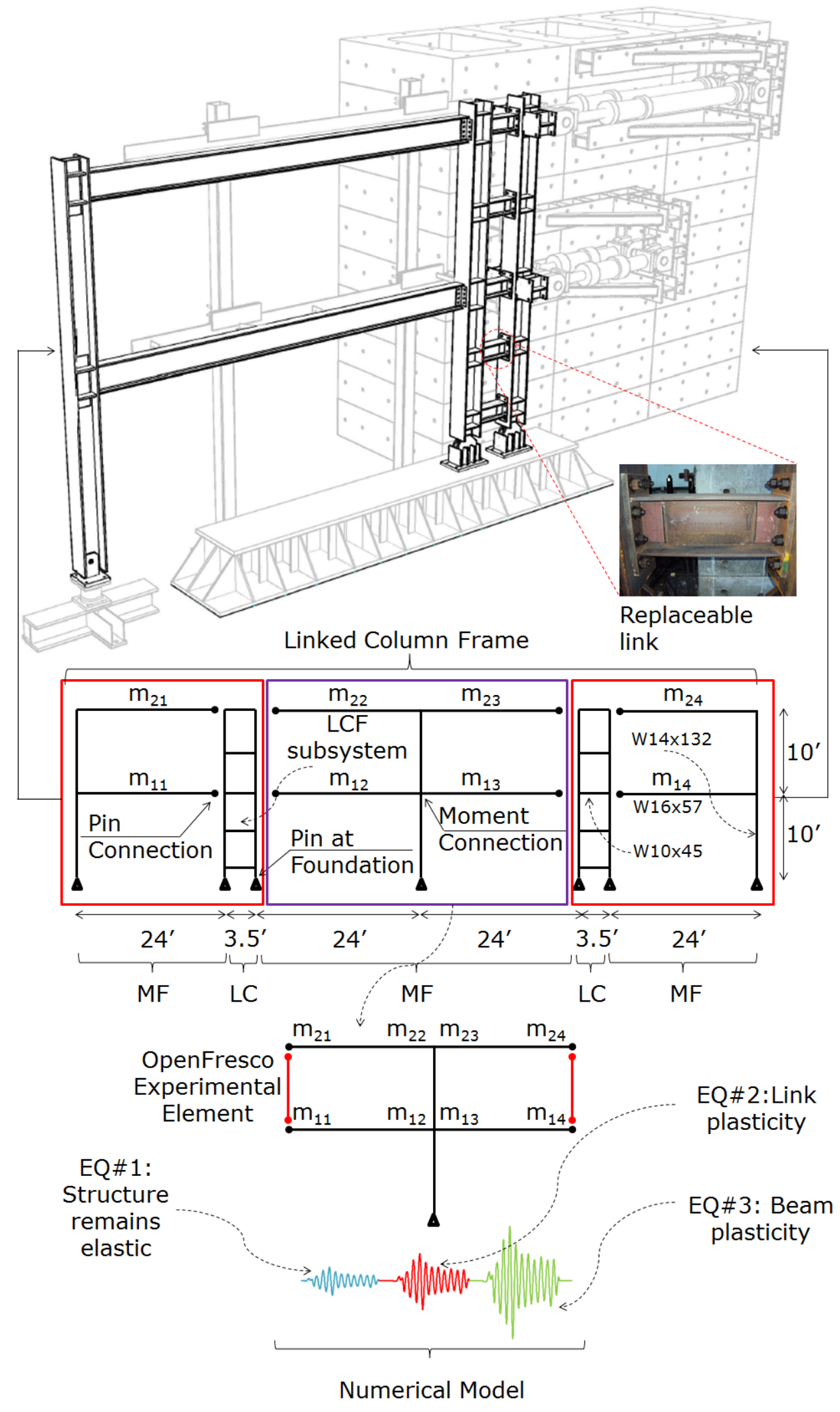

Figure 5.40: Hybrid simulation setup used on LCF-5L test assembly. 
The experimental substructure consisted of a 2-story LCF frame and 1-bay while the remainder of the building was numerically modeled. One of the goals of this research was to apply a set of ground motions, already discussed in Chapter (4), to the LCF in order to understand the behavior of the system during an earthquake event. Numerical investigation indicated that due to differences on lateral parameters between the multistory (2-story, 4-bay) and experimental (2-story, 1-bay) models, hybrid simulation is particularly well-suited for the experiment.

To perform the hybrid simulation, OpenSees was used as a finite element software to model and analyze the numerical part of the system, while OpenFresco was used as a middleware to connect the finite element analysis software with a control and data acquisition software. OpenFresco is an object oriented framework which pairs computational drivers with laboratory control systems to enable hybrid simulation. The OpenFresco experimental element, shown in Figure (5.40) acted within OpenSees to represent the portion of the structure that was physically tested in an experiment. Moreover, the experimental elements provided the necessary interface to the analysis procedures in the finite element analysis software.

To run a hybrid simulation test, four interacting components are required. The first component is a numerical model of the structure to be analyzed, which consisted of two gravity beams and one gravity column. The second component 


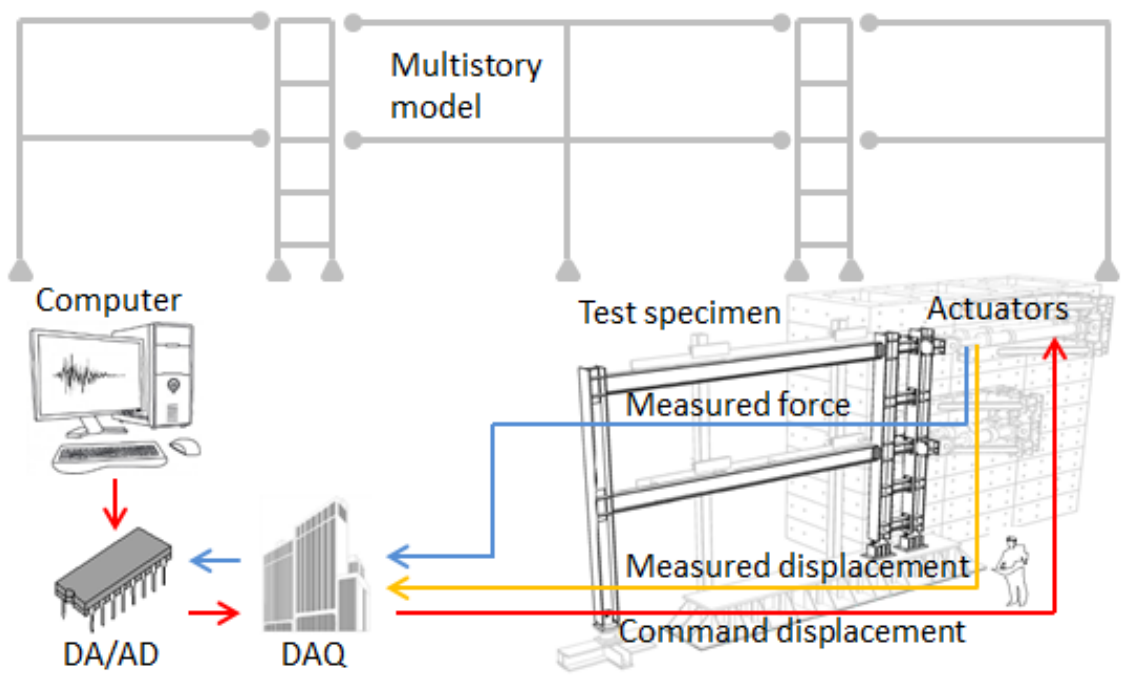

Figure 5.41: Interacting components of a hybrid simulation test.

is a servo-hydraulic control system with static or dynamic actuators that interacts with the specimen through structural response quantities such as forces and displacements. The third component is the physical test specimen itself (2-story, 1-bay), including reaction wall and strong floor. Finally, the fourth component is a data acquisition system with instrumentation that gathers all information for post-processing analyses. These interacting components are shown in Figure $(5.41)$

\subsection{Test Sequence}

Specimens LCF-5L and LCF-3L were both cyclically and hybrid tested. For evaluating the LCF response, FEMA 461 was used as the loading protocol for the cyclic testing, whereas ground motions at three different intensities; 50\%, $10 \%$ and $2 \%$ probability of exceedence in 50 years for Seattle, Washington were 
used for the hybrid testing. The system overall had exhibited three distinct performance levels; linearly elastic $(E)$, rapid return $(R R)$ to occupancy where only the replaceable links would yield, and collapse prevention $(C P)$ where the gravity beam components also become damaged. Table (5.2) summarizes the test sequence on the LCF test assembly.

Table 5.2: Test sequence on the LCF test assembly.

\begin{tabular}{ccccc}
\hline LCF-5L specimen & Max. Drift (\%) & Start & End & Time \\
\hline Cyclic & 2.6 & $10: 05 \mathrm{PM}$ & $1: 40 \mathrm{AM}$ & $3 \mathrm{~h} 35 \mathrm{~min}$ \\
Hybrid E & 0.5 & $2: 05 \mathrm{AM}$ & $2: 36 \mathrm{AM}$ & $31 \mathrm{~min}$ \\
Hybrid RR-1 & 2.0 & $4: 05 \mathrm{AM}$ & $5: 29 \mathrm{AM}$ & $1 \mathrm{~h} 24 \mathrm{~min}$ \\
Hybrid CP-2 & 4.5 & $5: 43 \mathrm{AM}$ & $6: 09 \mathrm{AM}$ & $26 \mathrm{~min}$ \\
\hline LCF-3L specimen & Max. Drift $(\%)$ & Start & End & Total time \\
\hline Hybrid E & 0.5 & $6: 40 \mathrm{PM}$ & $7: 03 \mathrm{PM}$ & $23 \mathrm{~min}$ \\
Hybrid RR-1 & 1.6 & $7: 11 \mathrm{PM}$ & $8: 35 \mathrm{PM}$ & $1 \mathrm{~h} 24 \mathrm{~min}$ \\
Hybrid RR-2 & 1.8 & $8: 47 \mathrm{PM}$ & $10: 15 \mathrm{PM}$ & $1 \mathrm{~h} 28 \mathrm{~min}$ \\
Hybrid CP-1 & 2.4 & $10: 24 \mathrm{PM}$ & $12: 45 \mathrm{AM}$ & $2 \mathrm{~h} 21 \mathrm{~min}$ \\
Hybrid CP-2 & 2.8 & $12: 53 \mathrm{AM}$ & $4: 10 \mathrm{AM}$ & $3 \mathrm{~h} 17 \mathrm{~min}$ \\
Ultimate Cyclic & 5.0 & $5: 15 \mathrm{AM}$ & $9: 05 \mathrm{AM}$ & $3 \mathrm{~h} 50 \mathrm{~min}$ \\
\hline
\end{tabular}

Since this was the first time that a LCF system was being tested, experimental tests for LCF-5L specimen started with a cyclic test up to $2.6 \%$ drift followed by a set of hybrid simulation tests reaching a $4.5 \%$ total drift limit at the end of about 6 hours of testing. During ground motion CP-2, one of the actuators, attached to the first floor, interlocked due to maximum force, abruptly stopping the test with only 26 minutes of ground motion. The ability to rapidly return 
to occupancy was achieved, between LCF-5L and LCF-3L tests, through replacing sacrificial removable links as the gravity system remained elastic. With the knowledge gained from specimen LCF-5F, a second round of experimental tests were conducted on the LCF-3L specimen, starting with hybrid testing up to $2.8 \%$ drift followed by an ultimate cyclic test until a $5.0 \%$ total drift limit was achieved. The total time of the experimental tests for LCF-3L was about 13 hours. The total time for each experimental test did not include the time for troubleshooting and inspection after each major event. 


\section{Chapter 6}

\section{LCF System Performance Validation}

\subsection{Introduction}

Cyclic and hybrid simulation tests of the linked column frame frame were conducted using the loading protocol and hybrid model described in Chapter (5). This section presents results that validates the performance of the LCF system. The specimens are presented in the order in which they were tested and some key aspects are discussed. Each test was conducted over the course of several days. At key points during the experiments, actuators were paused for visual inspection and photography. A friction test was conducted after removing shear

links and gravity beams for LCF-3L specimen. The same loading protocol used for the ultimate cyclic loading was used in the friction test in order to maintain the actuators speed per cycle. Results from Figure (6.1) indicate that friction forces around $10 \%$ of the total base shear were introduced during the experimental tests and all plots that use actuator forces were updated accordingly. To the knowledge of the author, the tests that were conducted in this research 


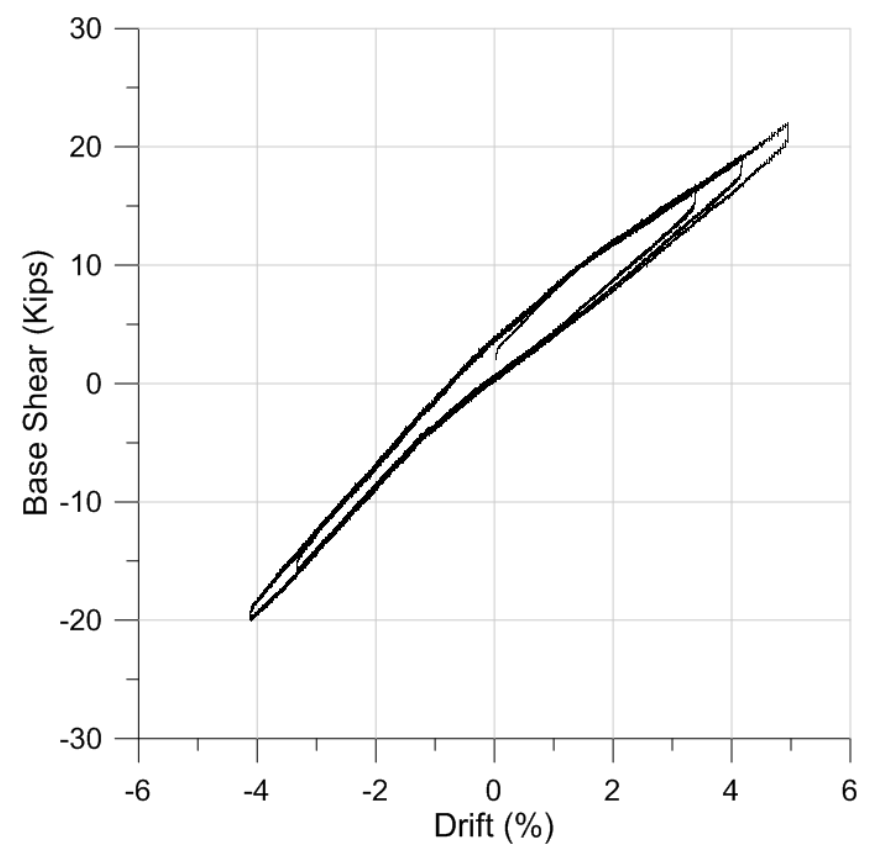

Figure 6.1: Friction test: Base shear versus overall drift.

were the first series of experiments specifically aimed at studying the seismic performance of full scale LCF systems constructed using realistic detailing.

\subsection{Initial Cyclic Behavior Evaluation}

Since this was the first time a LCF system was experimentally tested, a cyclic behavior evaluation was performed prior running the earthquakes via hybrid simulation. The main objective of the cyclic test was to understand how the system would interact together as a unit and shed light to subsequent tests. The idealized pin connection at foundation levels was studied in regards to base rotation. A LVDT was positioned on each foundation element and rotation was obtained from the LVDT values divided by distance from the LVDT itself to 
the center line of the pin connection. This procedure was done to linked and gravity columns. Figures (6.2) and (6.3) show the rotation at the base versus base shear on linked column 1, hereafter denoted as LC1, and rotation at the base versus base shear on gravity column, hereafter denoted as GC, respectively. The maximum base shear achieved was 200 kips while the maximum rotation was about 0.05 rad. Both LC1 and GC had shown a similar behavior indicating a pinching on their hysteresis plots due to a gap formed between the pin and connection plates indicating that the machining of these elements were not properly done.

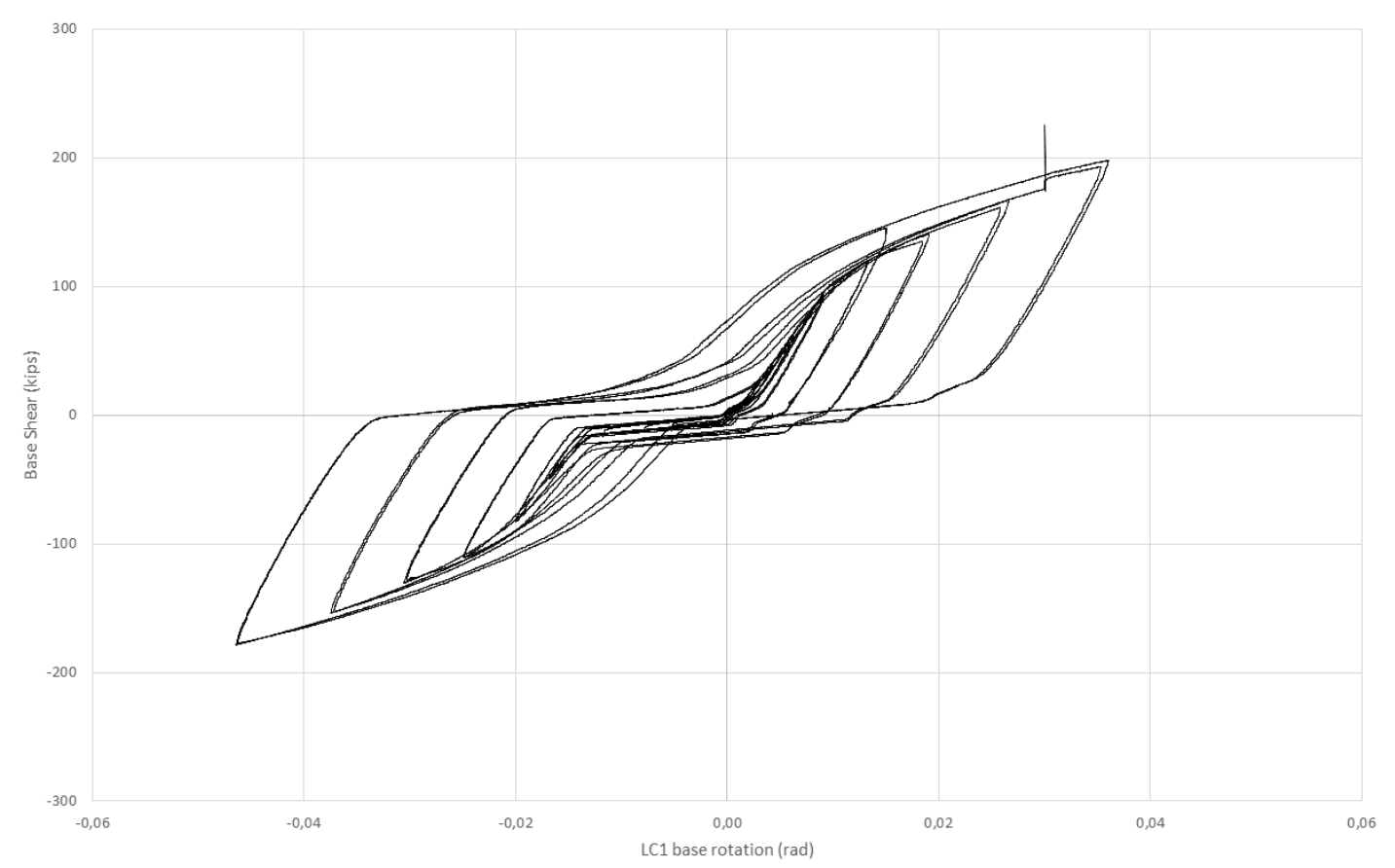

Figure 6.2: Base shear vs LC1 base rotation for LCF-5L specimen.

A further investigation was done in order to find out if the pinching effect pertained to the system. This was done through studies on the rosette strain 


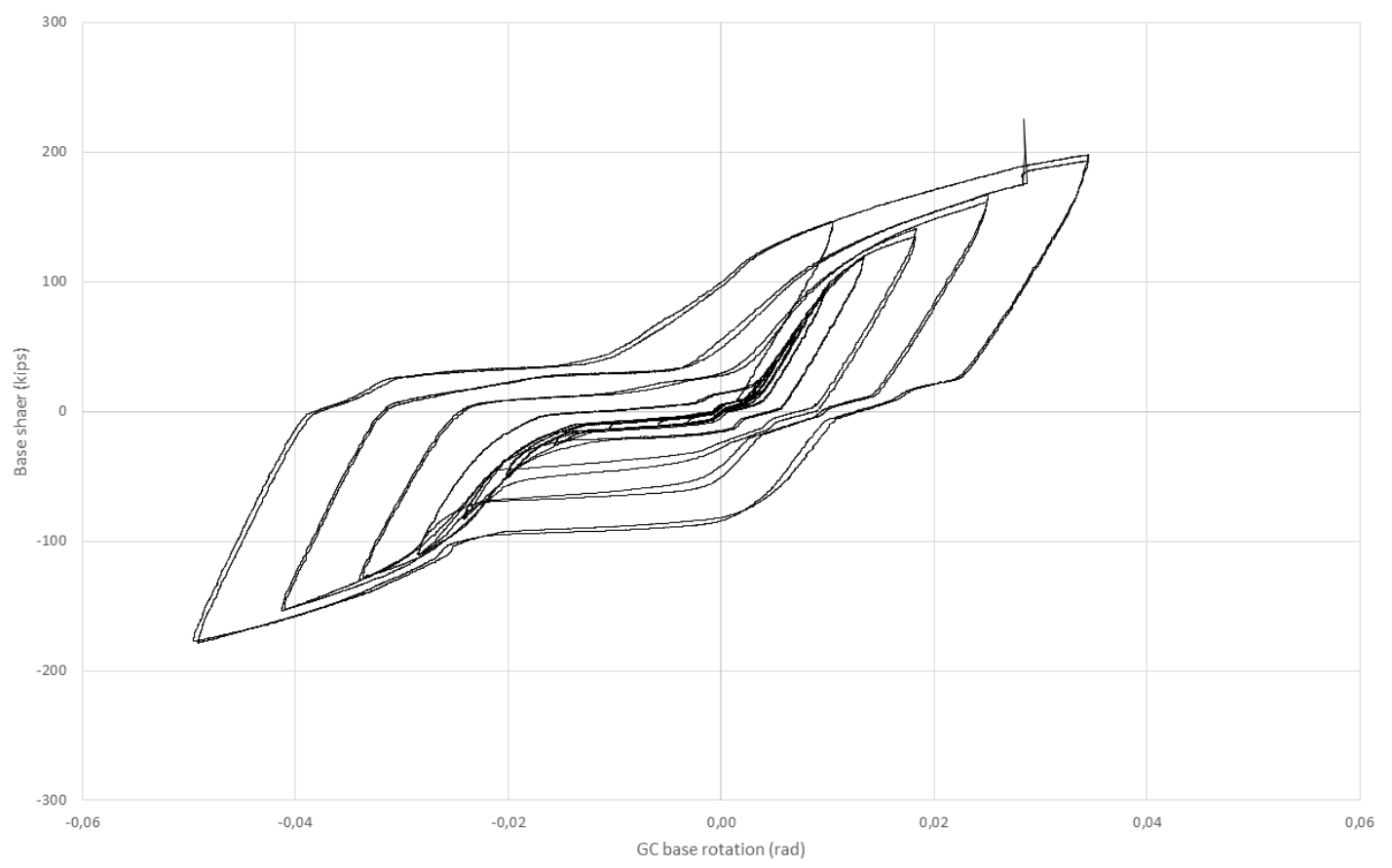

Figure 6.3: Base shear vs GC base rotation for LCF-5L specimen.

gage of Link 1 in order to obtain a limit for the shear strain $(\gamma=2960 \mu \mathrm{in} / \mathrm{in})$ and to capture the time when it occurred $(t=1158 \mathrm{sec}$. $)$. According to the failure theories one can express the critical value associated with each theory of failure either in terms of $\sigma_{y}$ or in terms of $\tau_{y}$. This means that the value of $\tau_{y}$ could be equal to 0.577 times the value of $\sigma_{y}$, according to Von Mises theory. Figure (6.4) shows the shear acting on both linked columns versus rotation of Link 1 based on results given by LVDTs. For $t=1158 \mathrm{sec}$. the results for shear and rotation were 116 kips and 0.0029 rad., respectively, and no pinching was observed when LVDT's were used to plot this performance metric.

Another important performance metric that was investigated during the 


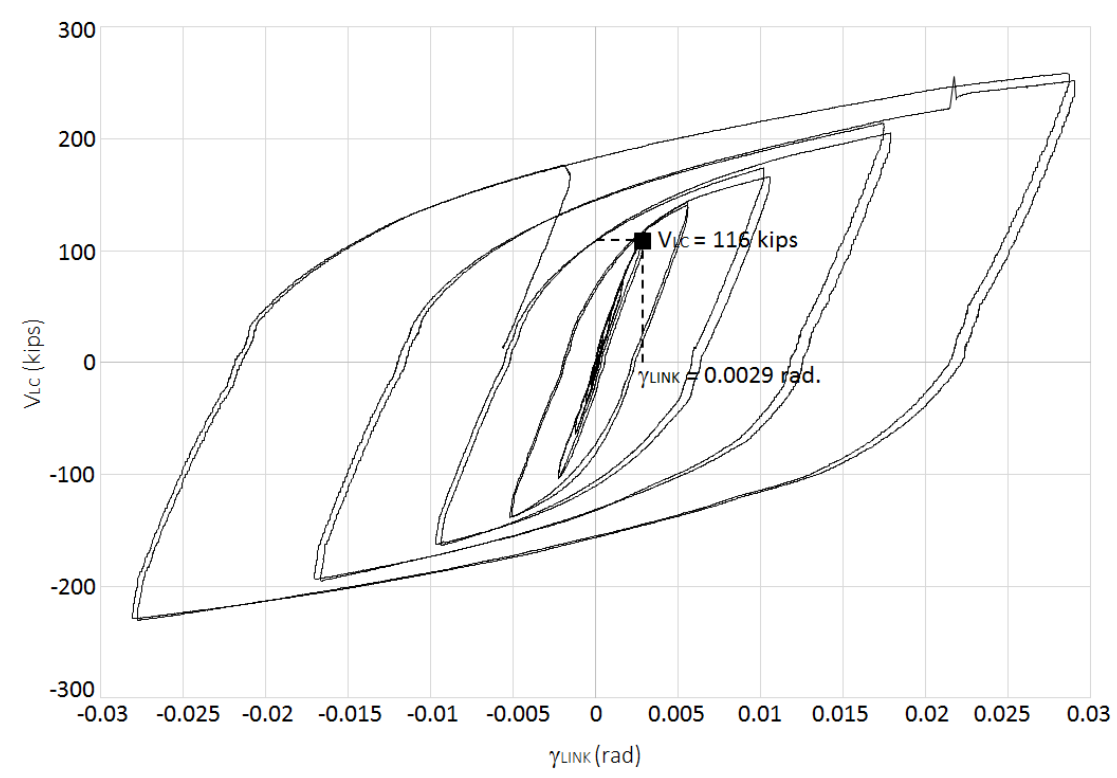

Figure 6.4: Shear on linked columns vs Link 1 rotation through LVDT.

initial cyclic test was related to the rotation of beam-column connections. Accelerometers were positioned at the center line of each column at both floor levels and rotation was obtained. Figures (6.5) and (6.6) indicate the base shear versus rotation on moment and shear connections, respectively. This moment connection is located at the node formed by the first floor and GC, whereas the shear connection is located at the node formed by the first floor and LC1. The behavior in terms of rotation follows the same pattern for both connections, suggesting that beam-to-column connections (moment side) used in the seismic force resisting system (SFRS) shall be capable of accommodating a story drift angle of at least $0.025 \mathrm{rad}$, whereas beam-to-column connections (shear side) used in the SFRS shall be capable of accommodating also a story drift angle of at least $0.025 \mathrm{rad}$. As a result, this suggests that connection details could be 
the same as intermediate moment frames.

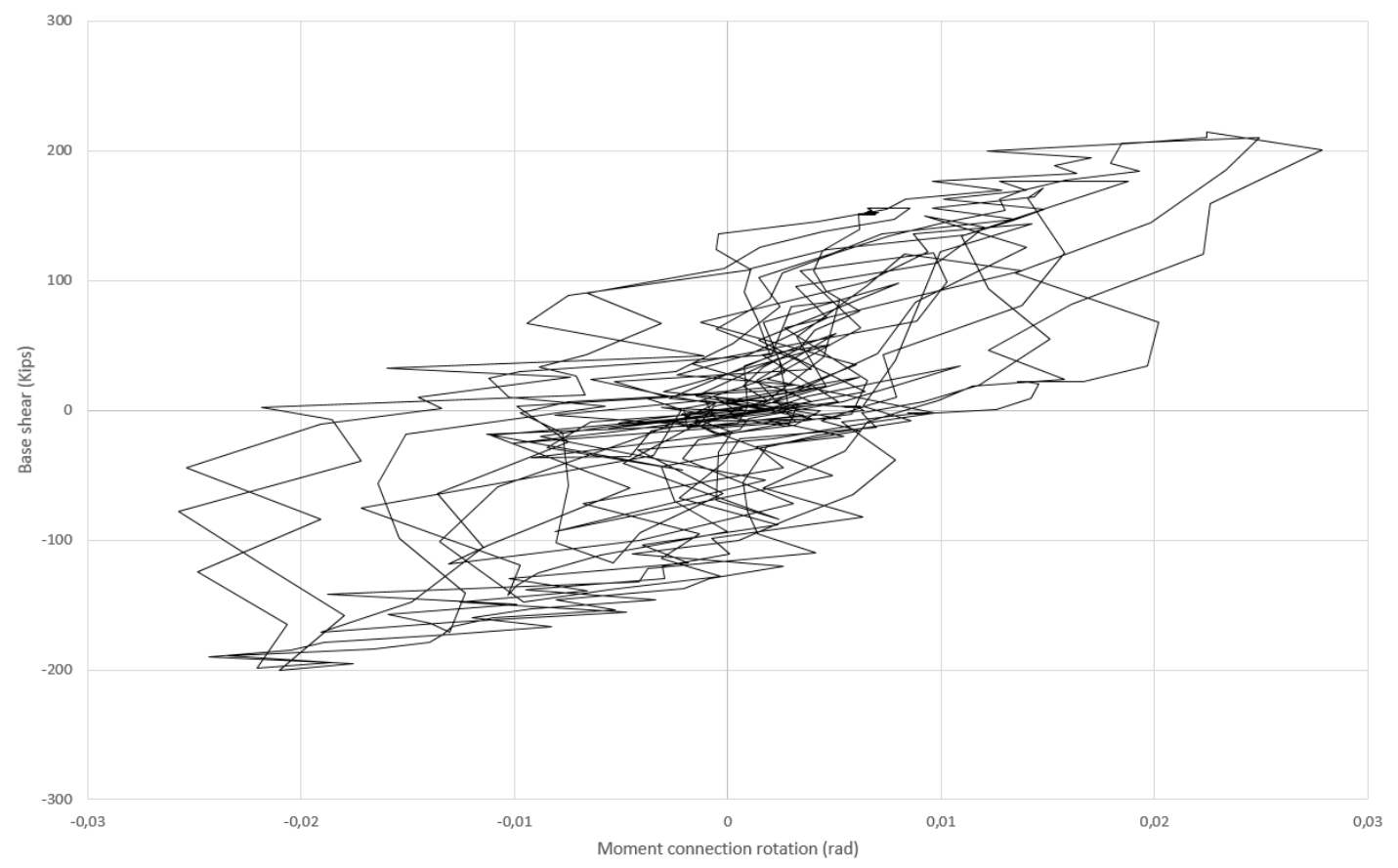

Figure 6.5: Base shear vs moment connection rotation for LCF-5L specimen.

One of the main objectives of this dissertation was to experimentally find the overstrength coefficient, $\Omega_{0}$, which is a seismic performance factor. Figure (6.7) shows the cyclic base shear versus drift for the LCF-5L specimen tested. The LCF-5L system was designed to have a base shear closer to the design base shear at first link yield. The nominal first significant yield occurred at $0.7 \%$ drift and $V_{\text {link }}=100$ kips for the base shear, whereas the prescribed minimum design seismic force level was calculated as $V_{\text {design }}=109$ kips. The ratio in lateral strength $\left(\Omega_{D}\right)$ between $V_{\text {link }}$ and $V_{\text {design }}$ is close to unity. The material overstrength $\left(\Omega_{M}\right)$ provides an allowance for strain hardening, where yielding is expected to occur. Code requirements have historically used a factor that 


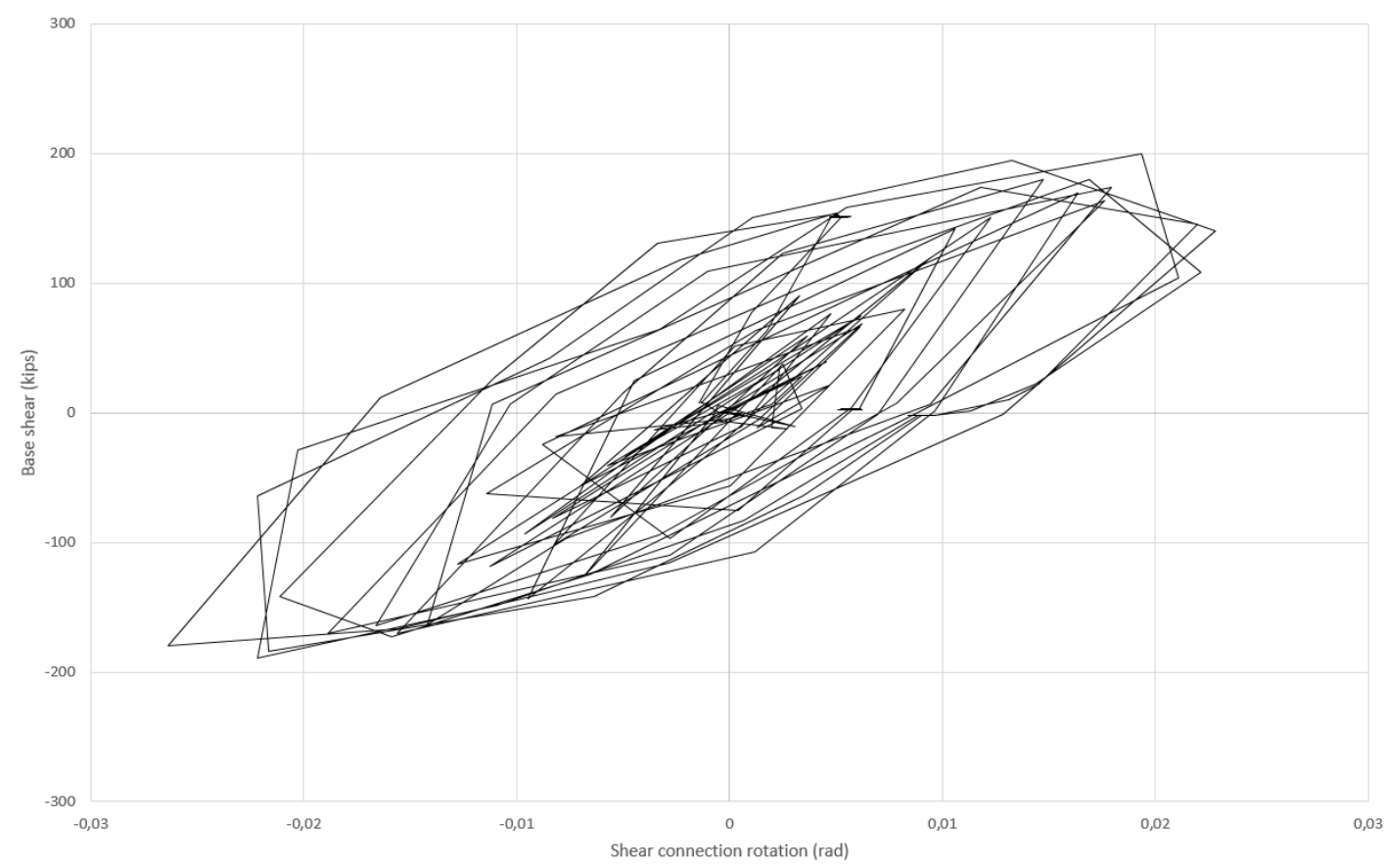

Figure 6.6: Base shear vs shear connection rotation for LCF-5L specimen.

varies from 1.15 to 1.25 . The first beam yield happened at $2.5 \%$ drift and $V_{\text {beam }}=199$ kips. The system overstrength factor $\left(\Omega_{0}\right)$ could be defined as a characteristic of a structural system where the actual strength is greater than the design strength. This factor accounts for overstrength of the structure in the inelastic range. For purpose of comparison, the overstrength factor used in ASCE/SEI 7 (2010), for special moment resisting frame and eccentrically braced frame, are $\Omega_{0}=3.0$ and $\Omega_{0}=2.5$, respectively, whereas for the LCF tested is $\Omega_{0}=2.0$. This factor might be considered to provide a reasonable estimate of the reserve strength attributable to a rapid return to occupancy level of performance. As mentioned before, the pinching in the hysteresis loops shown in Figure (6.7) was caused mainly by the lack of fit between the pin and the 
plates on the LC foundation element. The pinching effect is not related to the LCF system behavior whatsoever as can be seen in Figure (6.4). No sudden loss of stiffness on LCF system was observed.

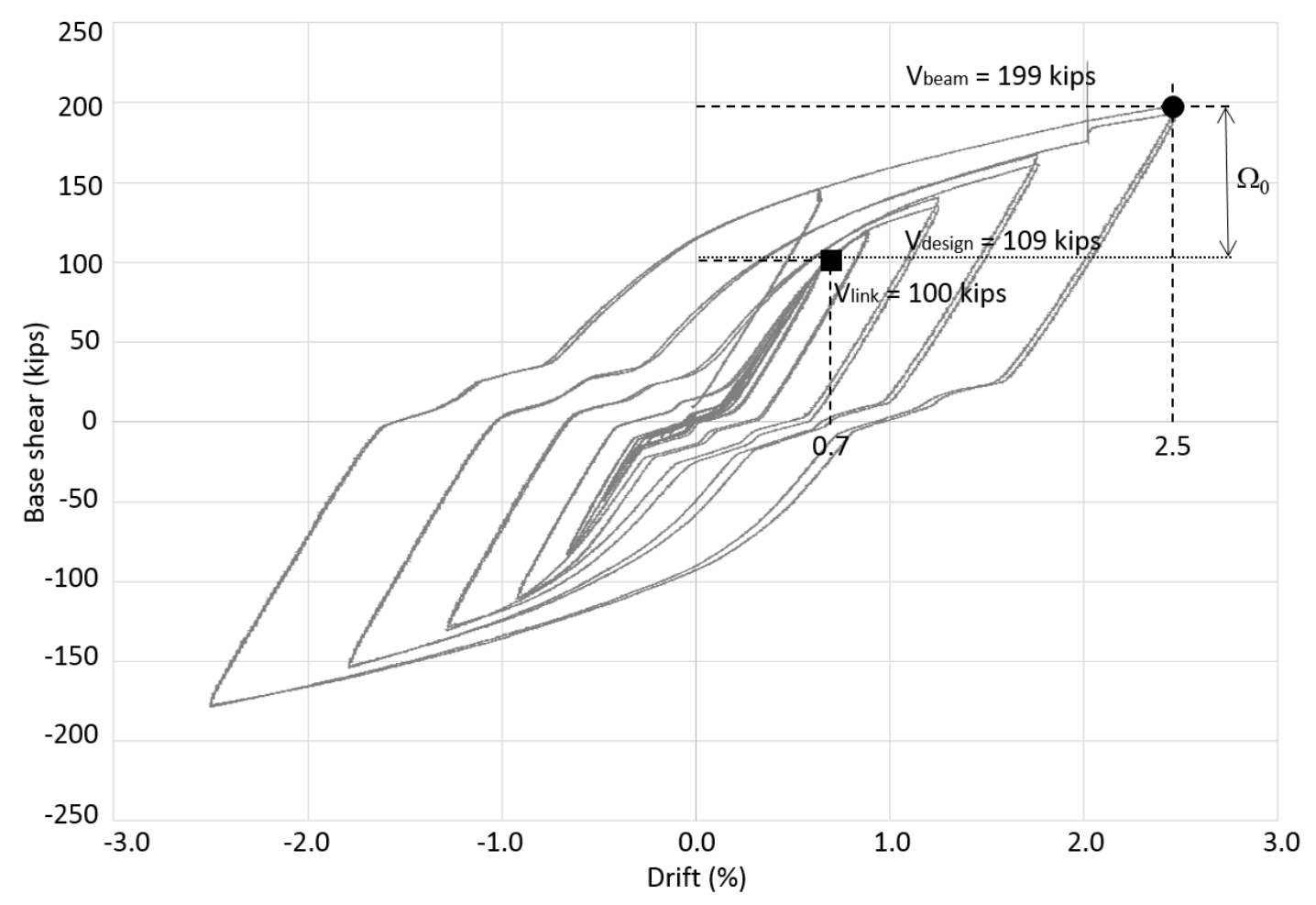

Figure 6.7: LCF system overstrength.

\subsection{Seismic Response of LCF-5L}

In order to proceed with the hybrid simulation tests, structural properties such as mass, damping and stiffness parameters were needed. Seismic masses were the same as those from the SAC 3-story building, $65.53 \mathrm{kips}-\mathrm{sec}^{2} / \mathrm{ft}$ for the first floor and $70.90 \mathrm{kips}^{-\mathrm{sec}^{2}} / \mathrm{ft}$ for the second floor. Lump masses were assigned at 
the nodes of each floor level as shown in Figure (6.8). Masses on the LCF subsystems were transferred to the hybrid simulation model where $m_{i j}$ indicates mass at floor $i$ located at bay $j$.
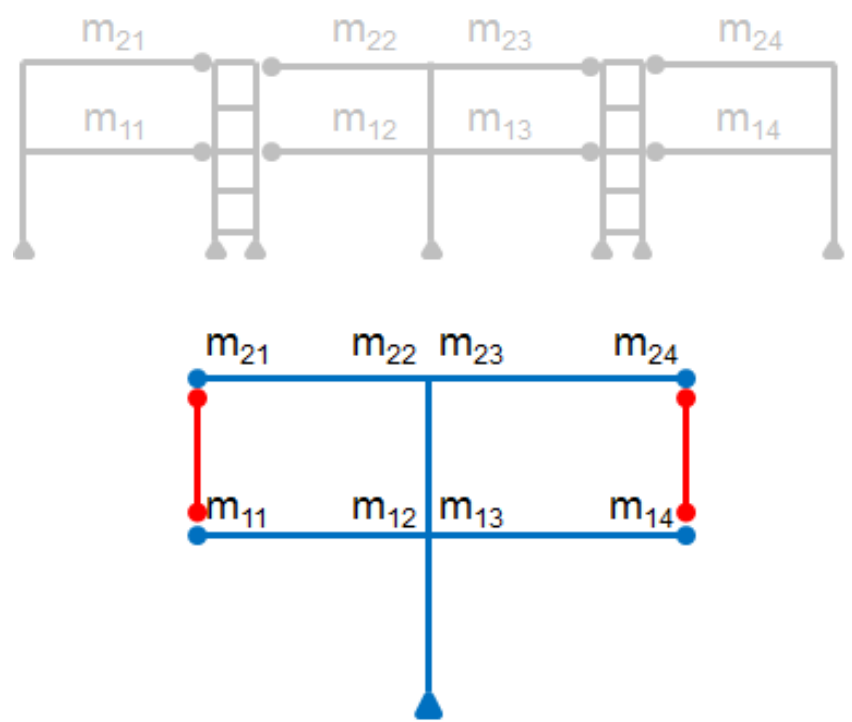

Figure 6.8: Lump masses for hybrid simulation test.

Rayleigh damping parameters were set equal to 0.02 for the first mode and 0.06 for the second mode of vibration. Newmark method for hybrid simulation with fixed number of iteration was used. The factors $\gamma=0.5$ and $\beta=0.25$ in the Newmark integrating scheme were also used.

For the stiffness parameters, a stiffness test was conducted on the LCF-5L specimen. Actuators were set to a predetermined force value and then displacements were calculated at each floor level to find out the stiffness matrix of the specimen. Figure (6.9) shows the coefficients of the stiffness matrix for the LCF5L specimen, while Equations (6.1) and (6.2) indicate the stiffness matrices for 
the LCF-5L specimen and for the hybrid simulation model, respectively, where $K_{i j}$ indicates level $i$ force versus level $j$ displacement.
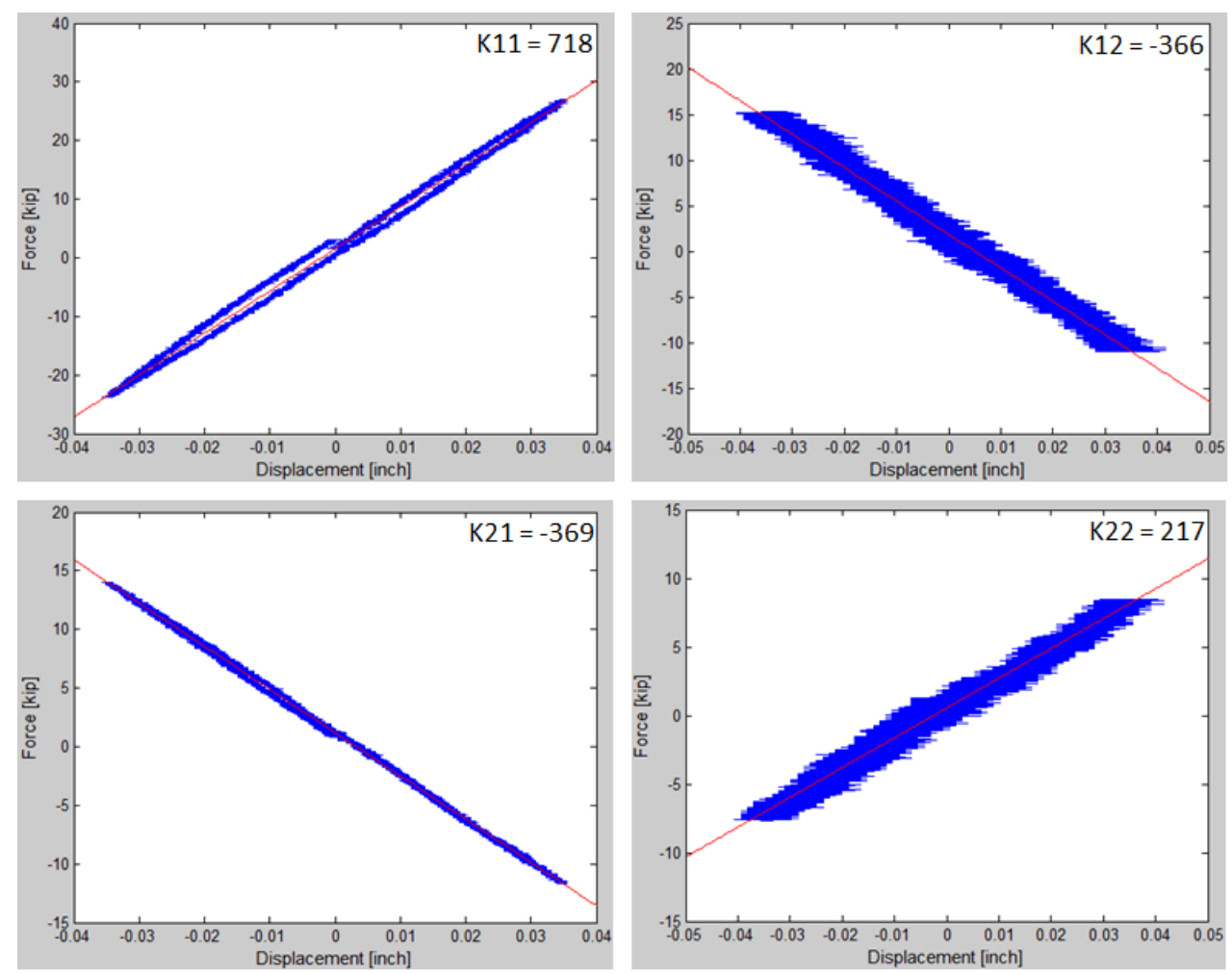

Figure 6.9: Stiffness test results for LCF-5L specimen.

$$
\begin{gathered}
{\left[k_{L C F-5 L}\right]=\left[\begin{array}{cc}
718 & -367.5 \\
-367.5 & 217
\end{array}\right]} \\
{\left[k_{\text {Hybrid }}\right]=\left[\begin{array}{cc}
1436 & -735 \\
-735 & 434
\end{array}\right]}
\end{gathered}
$$


Once the structural properties are obtained, a family of time-stepping methods based on Newmark's method is used. The set of Equations (6.3) provide the basis for computing $u_{i+1}, \dot{u}_{i+1}$ and $\ddot{u}_{i+1}$ at time $i+1$ from the known displacement, $u_{i}$, velocity, $\dot{u}_{i}$, and acceleration, $\ddot{u}_{i}$, at time $i$, where $M$ is the mass matrix, $C$ is the Raleigh damping matrix and $K$ is the stiffness matrix.

$$
\begin{array}{r}
M \ddot{u}_{i+1}+C \dot{u}_{i+1}+K u_{i+1}=p_{i+1} \\
u_{i+1}=u_{i}+\Delta t \dot{u}_{i}+\frac{(\Delta t)^{2}}{2}\left[(1-2 \beta) \ddot{u}_{i}+2 \beta \ddot{u}_{i+1}\right] \\
\dot{u}_{i+1}=\dot{u}_{i}+\Delta t\left[1-\gamma \ddot{u}_{i}+\gamma \ddot{u}_{i+1}\right]
\end{array}
$$

A total of three earthquakes (EE, RR-1 and CP-2) were used on the LCF-5L specimen. The application of the ground motions revealed a sequence of yielding events as follows: link 1 at base, link 2 at mid-height first story, link 3 at first story, link 4 at mid-height second story, link 5 at second story, GB at first story, and GB at second story. During the experiment, one of the actuators force capacity was reached in the push direction at $t=6240 \mathrm{sec}$ on the final earthquake run, collapse prevention level, and the test was abruptly stopped. However, a maximum drift of $4.2 \%$ could be achieved.

Axial loads on linked columns were investigated. LC1 had twelve strain gages attached to each face of the column, whereas LC2 had ten strain gages 
attached to each face of the column. In order to find the axial loads, a set of strength of materials equations was used as follows:

$$
\sigma=\frac{P}{A} \pm \frac{M \cdot y}{I}
$$

where $\sigma$ is the column stress, $P$ is the unknown axial load, $A$ is the area of the column, $M$ is the bending moment, $y$ is the distance from the neutral axis to the outer fiber, and $I$ is the moment of inertia. Using Hooke's law and eccentricity, $e$, Equation (6.4) can be written as:

$$
\epsilon . E=\frac{P}{A} \pm \frac{P . e . y}{I}
$$

where $E$ is the longitudinal modulus of elasticity. Therefore, axial load is given by:

$$
P=\frac{\epsilon \cdot A \cdot E \cdot I}{I \pm e \cdot y \cdot A}
$$

The LC pinned connections were designed for the maximum forces generated by the shear links. As explained in the AISC Seismic Provisions (ANSI/AISC $341,2010)$, an overstrength factor of 1.5 has generally been applied to the nominal strength of shear links $\left(V_{p}=102\right.$ kips $)$ to determine the design strength of the surrounding components. However, studies on shear link hysteretic energy dissipators (Dusicka et. al., 2004 ${ }^{b}$ ) indicate that the overstrength factor 
exceeded the expected overstrength for shear links by a wide margin. Thus, for design purposes, the overstrength factor used in the LCF system was 2.0.

Figures (6.10) and (6.11) show the seismic internal axial loads on LCs along its height as well as the Seismic Provisions and LCF design values for comparison. Experimental results indicate similar behavior on both columns and maximum values that corroborate with the overstrength LCF design factor of 2.0.

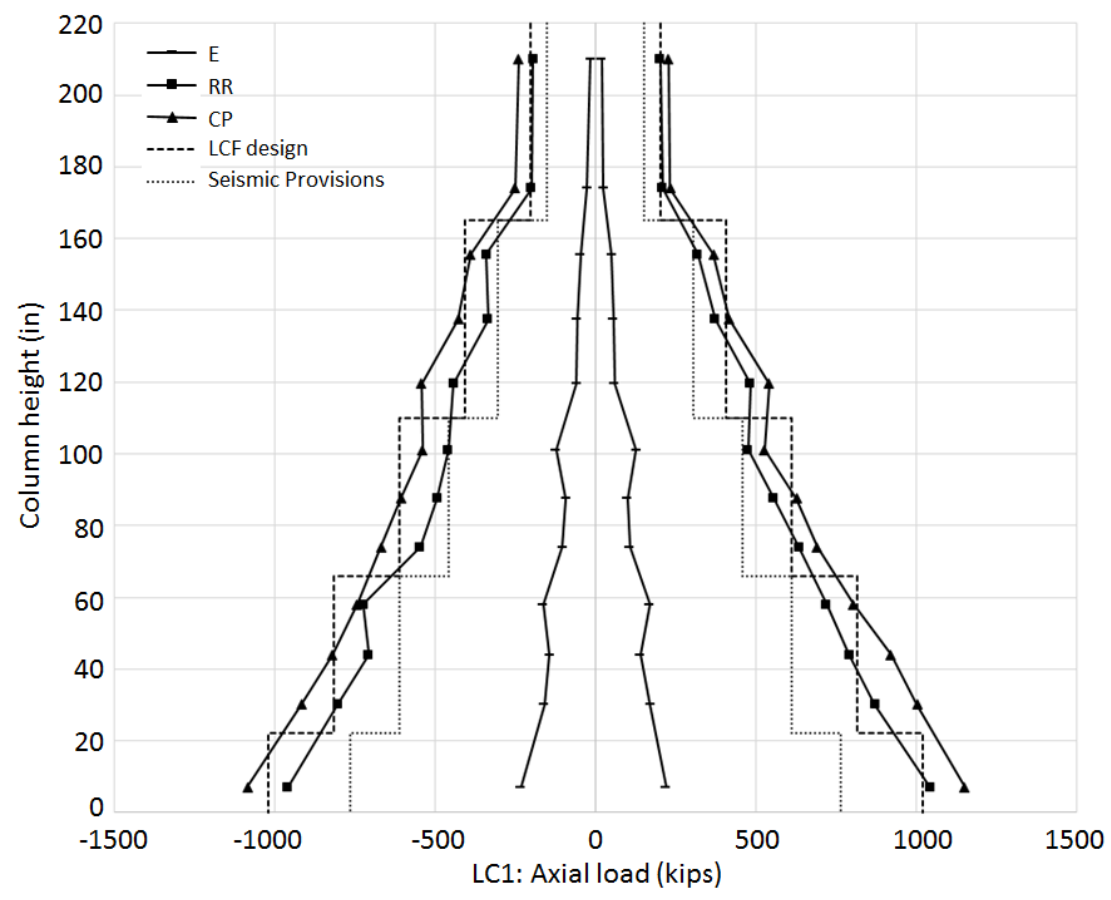

Figure 6.10: Axial loads along LC1 height for LCF-5L specimen

Figure (6.10) shows a well distributed axial load along the column and a maximum axial load on the foundation of about 1,200 kips. As expected, the increase of axial loads was obtained while changing the level of earthquake. Using Seismic provisions overstrength factor had shown that the structure might 
be underdesigned if this factor is used to design the foundation element. A similar behavior was obtained in Figure (6.11) with a maximum axial load on the foundation of about 1,300 kips. Even though an overstrength factor of 2.0 was used for the LCF design, a difference of about $27 \%$ was noted between the LCF design value and the maximum axial load on the linked columns, suggesting that a overstrength factor greater than 2.0 might be considered in the future, while considering the collapse prevention performance level.

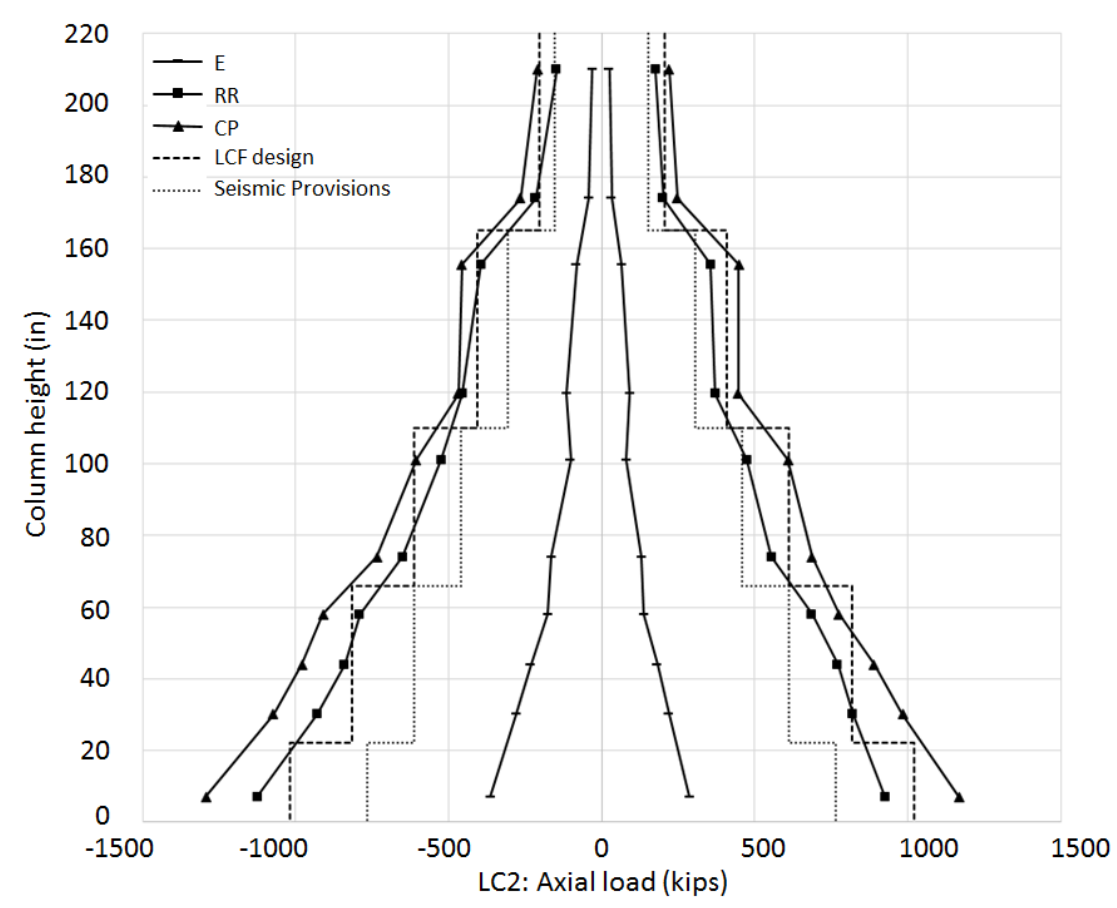

Figure 6.11: Axial loads along LC2 height for LCF-5L specimen

Axial loads and bending moments cause normal stresses on the column and both of them should be addressed. One of the advantages of the LCF system over other conventional systems is that, SMFRs, for example, allow plastic hinges on columns, while LCFs do not. In order to move closer to the goal 
of eliminating column damage, all columns in the LCF system were pinned at the foundation level and the strong-column and weak-beam approach was also used. The seismic internal bending moments were obtained for each level of earthquake using a similar approach that was used to obtain the axial loads. Figures (6.12) and (6.13) show the seismic internal bending moments on LCs along its height and experimental results indicate no damage on columns, with the plastic moment for a W14x132 column section being $M_{p}=975$ kips.ft while the maximum moment obtained on the LCs was about $M_{L C}=230$ kips.ft. As expected, figures (6.12) and (6.13) also show higher moments at lower level heights.

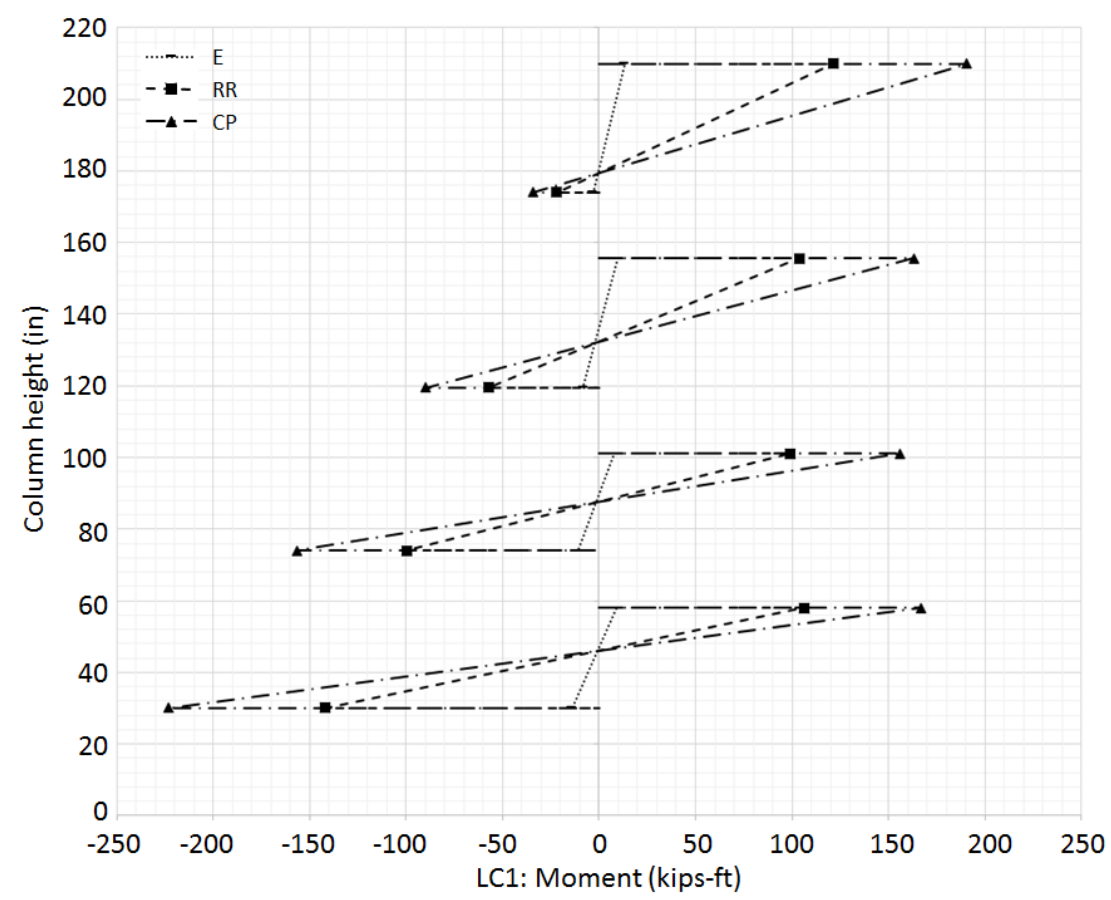

Figure 6.12: Moments at LC1 for LCF-5L specimen.

As explained in Chapter (3), the LCF system has also idealized physical 


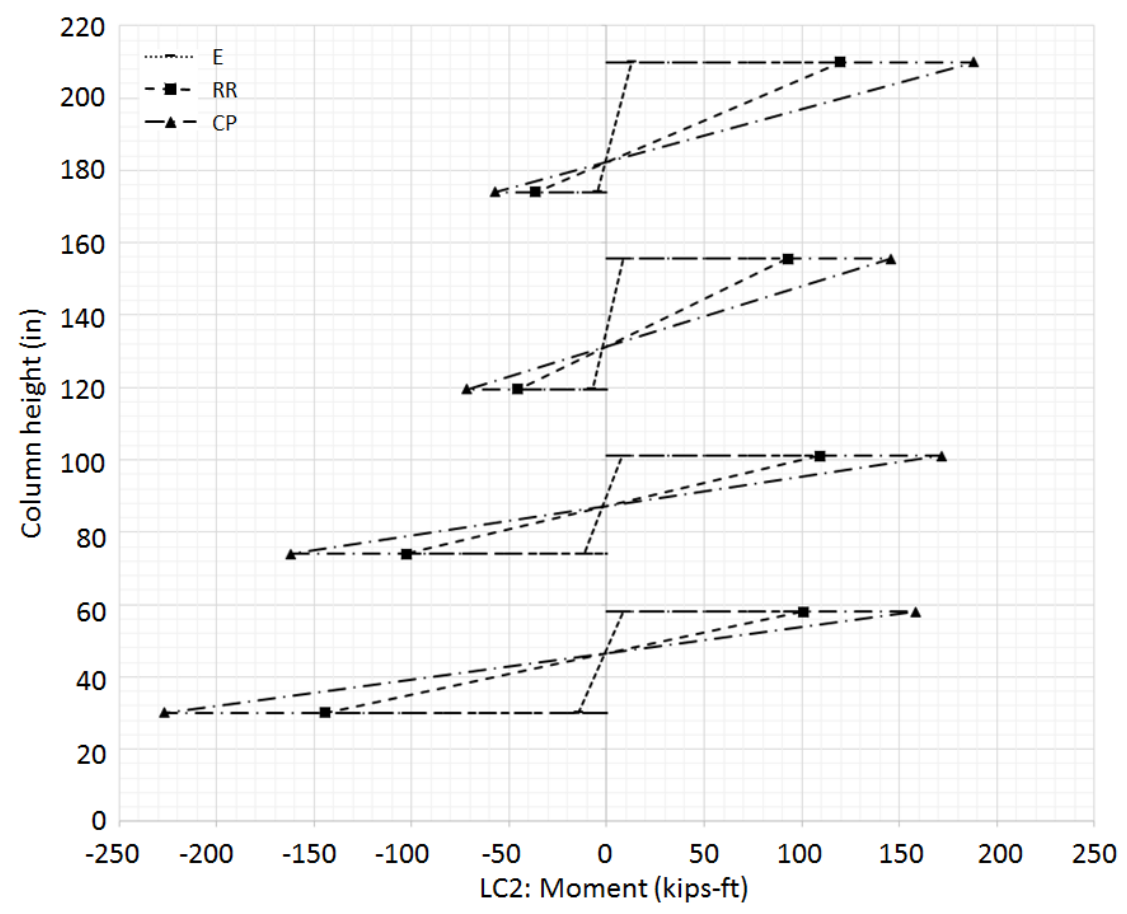

Figure 6.13: Moments at LC2 for LCF-5L specimen.

hinges while connecting the gravity beams to the linked columns. The outcome of this model is to achieve flexibility in the LCF system. Lowering the moment of inertia in the gravity beams is one means of achieving flexibility within the system. Another effective way is to change the boundary conditions of the gravity beams. For the LCF systems evaluated, a fully restrained moment connection at one end of the beam and a shear connection at the other end of the beam was used.

Figures (6.14) and (6.15) show the bending moments on the gravity beams and indicates a proper behavior of a fixed and pinned-end connections, respectively. As shown, the plastic moment for a W16x57 beam section was $M_{p}=5,775$ kips.in and did not occur until collapse prevention performance 
level was achieved. Figures (6.14) and (6.15) also shown the same behavior and clearly indicate an increase of bending moments while changing the levels of earthquake. Also, the shear connection behaved as expected with almost zero moment on its end. Results from bending moments on the gravity beams suggest that the LCF system is capable of achieving a rapid return to occupancy level of performance.

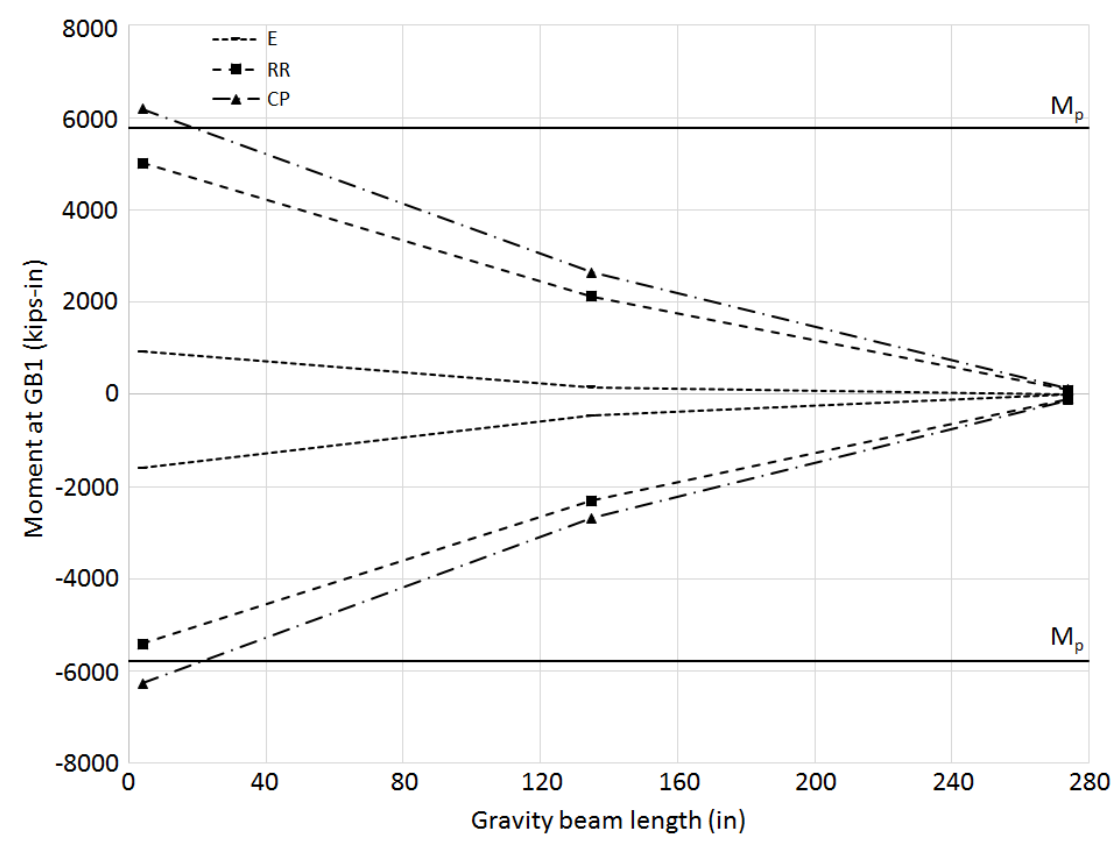

Figure 6.14: Moments at GB1 for LCF-5L specimen.

Another way to validate the LCF performance toward rapid return to occupancy is through the analysis of gravity beam flange strain distribution. Figure (6.16) shows the gravity beam flange strain distribution for the rapid return to occupancy and collapse prevention performance levels. The yielding strain $\gamma_{y}$ is shown by horizontal solid lines for reference and was obtained using Hookes 


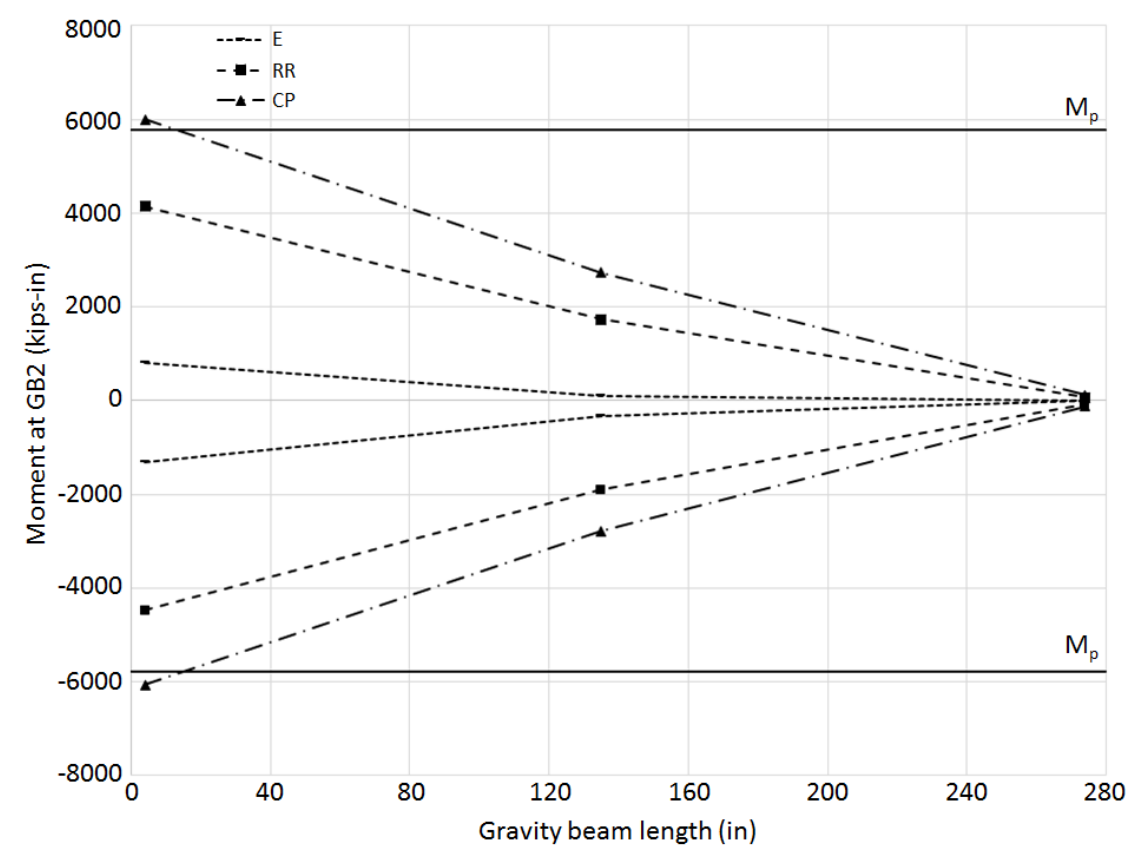

Figure 6.15: Moments at GB2 for LCF-5L specimen.

law. As shown, beam flange yielding does not occur until collapse prevention performance level is achieved. This ensures that no repairs would be necessary and will help to minimize post event repair costs. Figure (6.16) also shows limited demands on gravity beams and this could indicate less rigorous detailing connections. The behavior of both gravity beams was almost identical, except that strains for the collapse performance level on GB-1 was higher than GB-2.

After analyzing some system performance metrics, component level analyses were also analyzed. Since the replaceable shear links play an important role within the LCF system, a study on links' rotation along the column height is discussed. Figure (6.17) shows link rotation for all three performance levels for LCF-5L specimen. The link rotation demands in Link 1 at the base were greater 


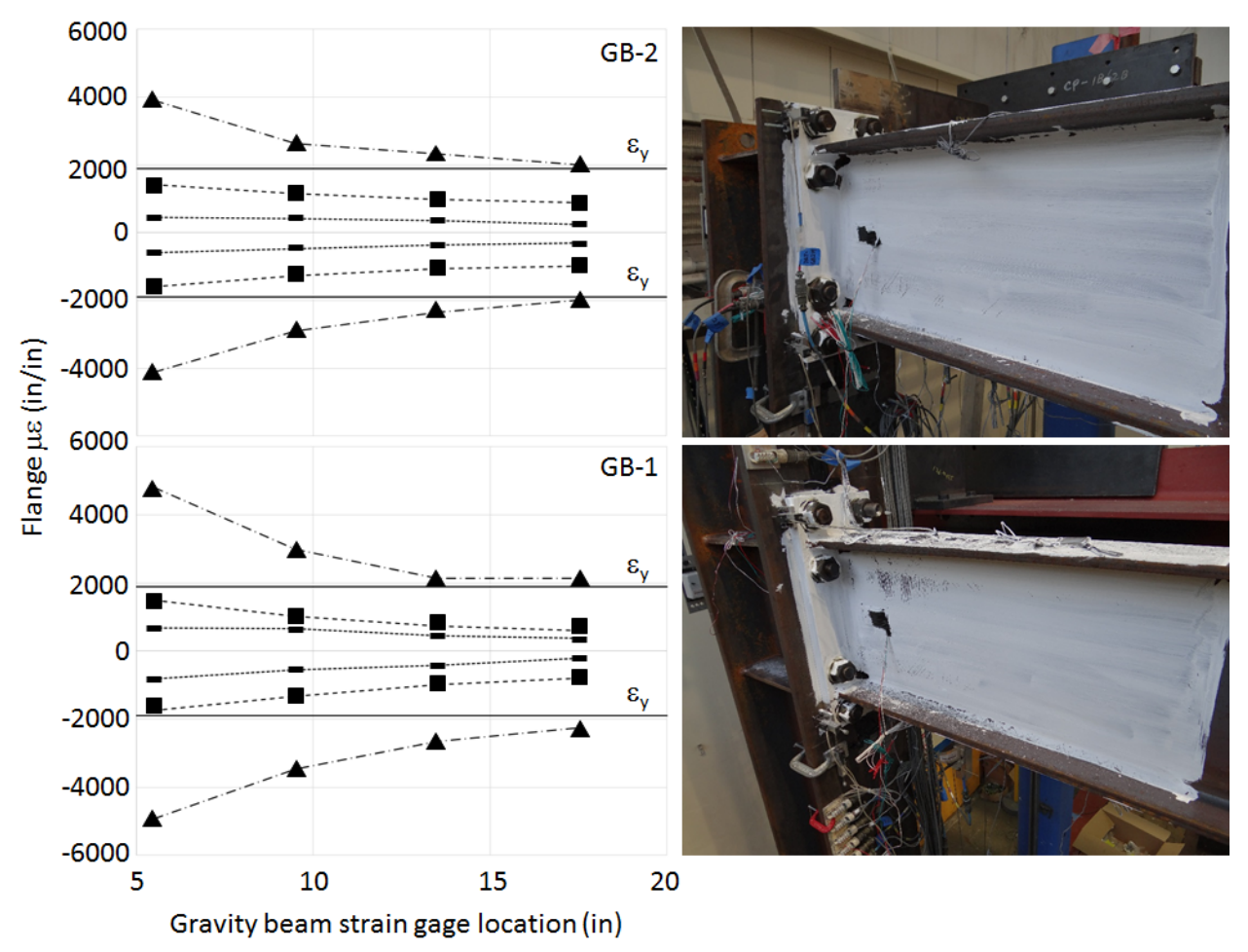

Figure 6.16: Gravity beam strain distribution for LCF-5L specimen.

than in Link 5 at the second story which concur with the sequence of yielding events mentioned previously. Whitewash on the links began flaking near midspan, then propagated toward the end plates as shown in Fig. (6.18). In the collapse prevention performance level the links have larger inelastic demand and are more likely to require replacement. The maximum rotation value on the RR performance level was $0.02 \mathrm{rad}$, whereas for the CP performance levels was about $0.06 \mathrm{rad}$. Cyclic results are also shown for a drift of $2.5 \%$, indicating results between the $\mathrm{RR}$ and $\mathrm{CP}$ performance levels. It should be noted that none of the links failed under any ground motions used in the hybrid simulation test. 


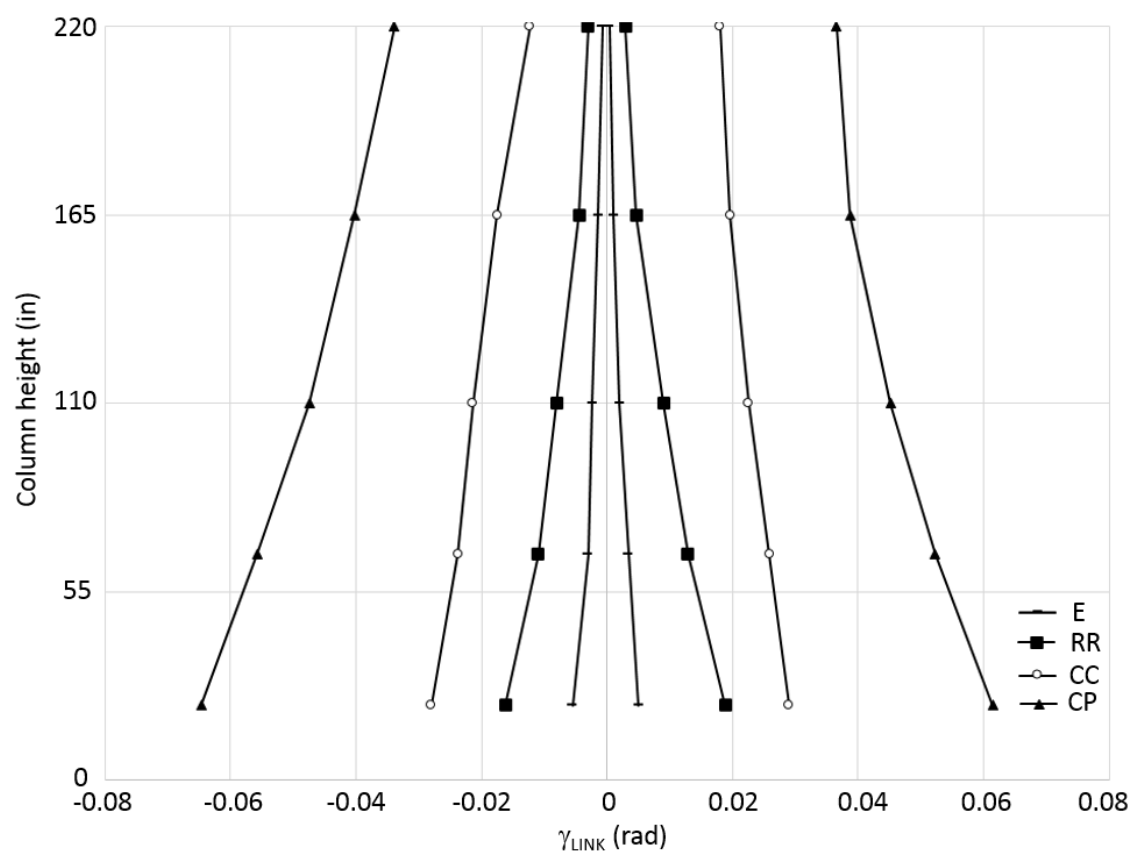

Figure 6.17: Link behavior along column height: LCF 5 links

Throughout the experimental program the panel zones behavior was continuously monitored. LVDTs were positioned diagonally over the panel zone and a strain gage rosette was attached to the column web. For each test, the maximum panel shear deformation, $\gamma_{\max }$, and the panel ductility, $\mu$, were determined. Ductility was calculated by dividing the absolute maximum panel shear deformation by the deformation at panel yield. Results for earthquake CP-2 are summarized in Table (6.1). As can be noted, all panel zones sustained loads higher than their nominal yield load. No weld fracture and no signs of distress or stiffness degradation were observed.

In addition to the analyses of axial loads on the linked columns, an evaluation of shear forces on the replaceable links was performed. Once the axial loads were 

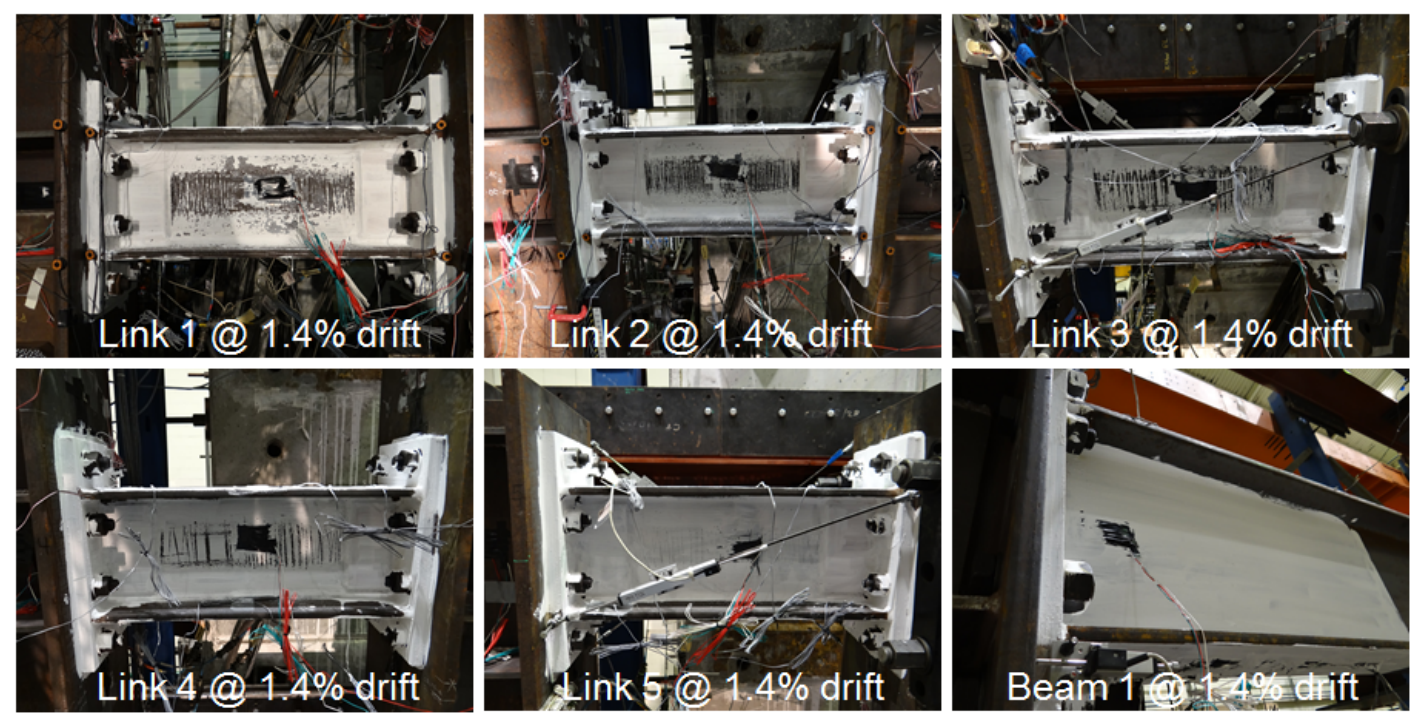

Figure 6.18: Damage in structural members at $1.4 \%$ drift.

Table 6.1: Panel Zone $\gamma_{\max }$ and location.

\begin{tabular}{cccc}
\hline Panel Zone & $\gamma_{\max }$ & $\mu$ & Location \\
\hline PZ-1 & 0.0043 & 1.45 & GC - 2nd floor \\
PZ-2 & 0.0036 & 1.22 & LC1 - 2nd floor \\
PZ-3 & 0.0048 & 1.62 & GC - 1st floor \\
PZ-4 & 0.0032 & 1.08 & LC1 - 1st floor \\
PZ-5 & 0.003 & 1.01 & LC1 - Link 1 level \\
\hline
\end{tabular}

determined through strain gage data, the next step was to calculate the shear on the replaceable links. This procedure was done taking the difference on axial loads between the column above and below the replaceable link. For example, the shear on Link 1 for the collapse prevention level was obtained from the difference between 1167 kips and 889 kips which corresponds to 278 kips. The same procedure was done to Links 2 and 4 . However, in order to capture the correct shear on the link located at the floor levels, Links 3 and 5, the shear from the gravity beam must be calculated as well. Figure (6.19) shows a free-body diagram to calculate shear on links. For example, the shear on Link 3 for the 
collapse prevention level was obtained from the difference between 607 kips and 390 kips which corresponds to 217 kips. This value needs to be subtracted from the gravity beam shear which is 20 kips. Therefore, shear on Link 3 is 197 kips. Note that the gravity beam moments were already obtained as indicated in Figures (6.14) and (6.15). Those moments are used to determine the shear on the gravity beam. Figure (6.20) summarizes the shear force on links for each level of earthquake.

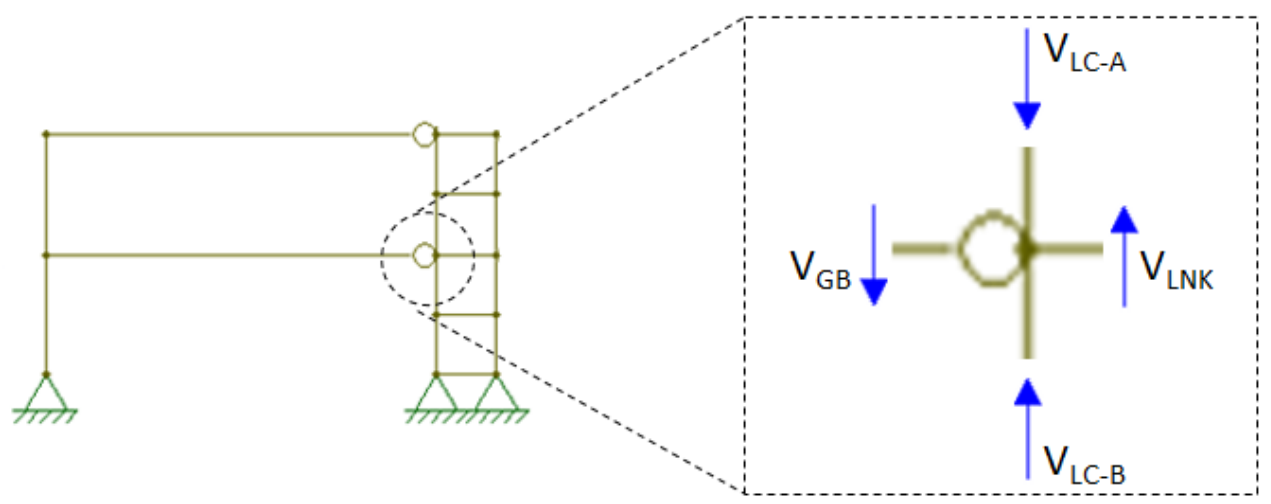

Figure 6.19: Free body diagram to calculate shear on links.

LCF-5L specimen exhibited three regions within the lateral response; elastic, yielding of links and yielding of links as well as MF beams. Provided the links are replaceable, these correspond to three distinct performance levels; elastic, rapid return to occupancy and collapse prevention. No significant permanent drift was observed during the earthquake motions. Figure (6.21) shows the experimental test setup after the hybrid testing for LCF-5L specimen. 


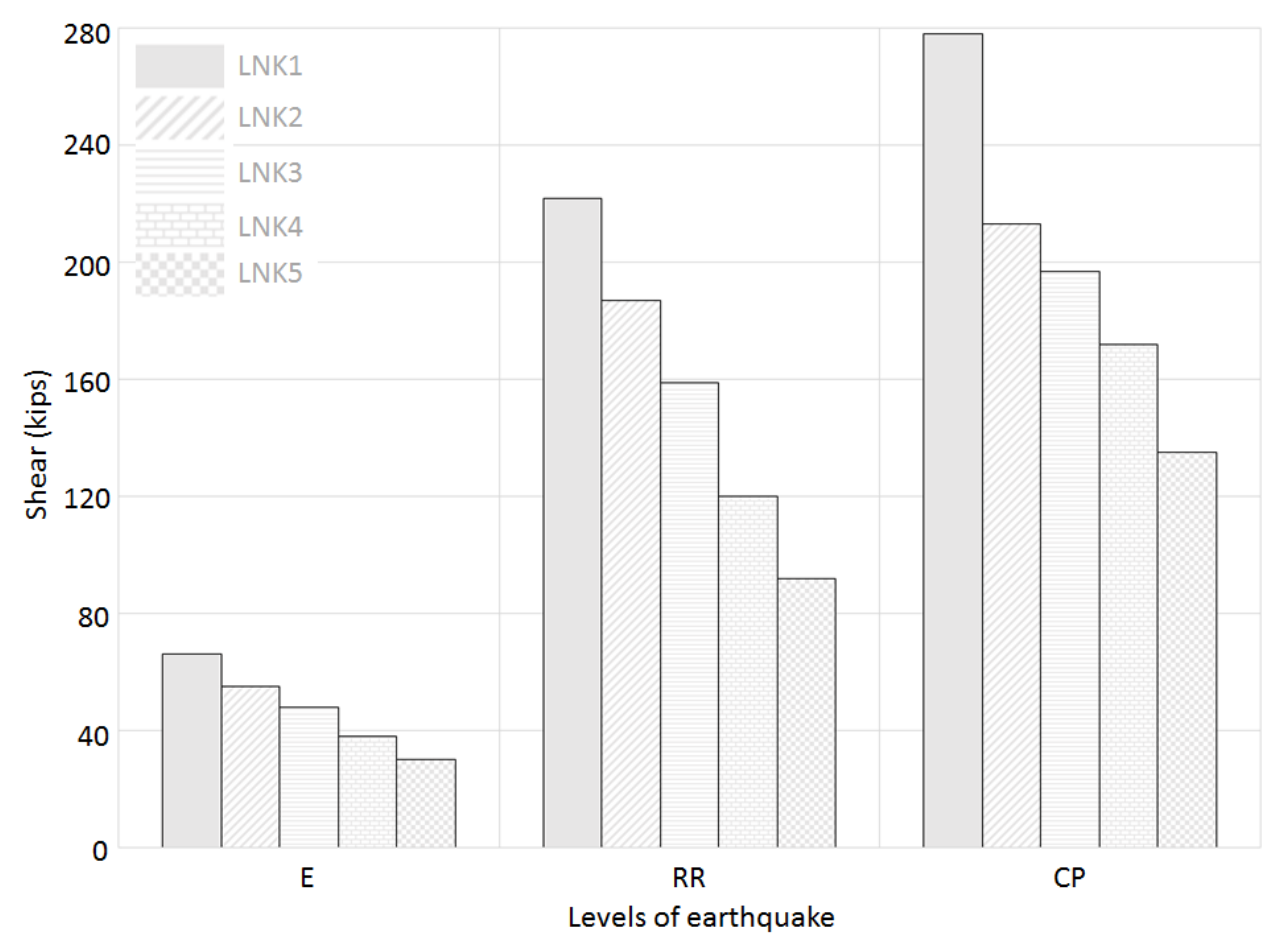

Figure 6.20: Shear on links vs levels of earthquake for LCF-5L specimen.

\subsection{Replaceability of Links}

Replaceability of shear links was performed after the hybrid simulation test for the LCF-5L specimen was done. Since the hybrid test aborted due to tripping a force interlock, the permanent displacement was rather large and unrealistic in terms of residual displacement. So, the actuators were brought to the zero position to get the frame as close to vertical as possible for the ease of links' replacement. And, also to straighten the gravity beams for the second test. All links were easily unbolted without the use of any torch, however, bolts were hammered out because they were jammed by the shear link. The lack of residual drift helped the process of replacing the links, this could prove more difficult 


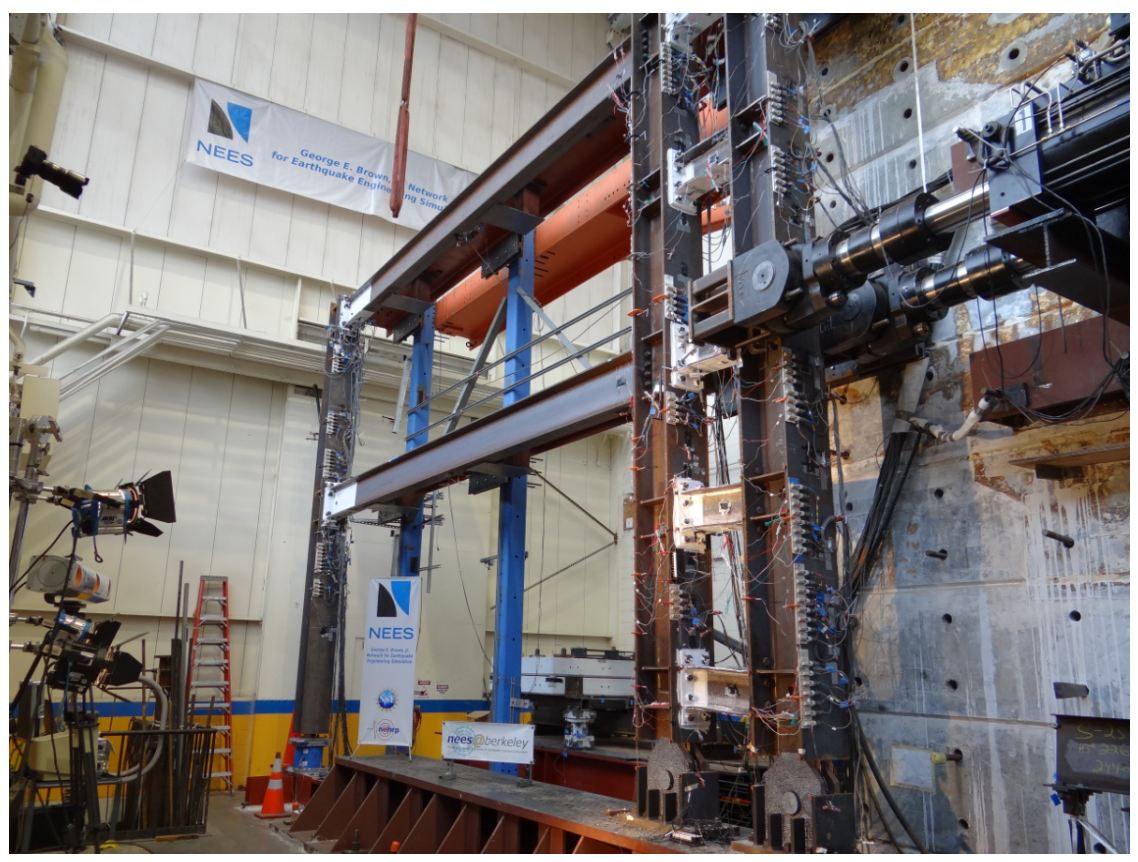

Figure 6.21: Experimental test setup after hybrid testing for LCF-5L specimen.

to achieve in a real building. According to McCormick et. al. (2008) a 0.5\% residual drift represents a limit beyond which it is more economical to rebuild a structure than it is to repair it. Nevertheless, even when residual drift results in the structure, the permanent deformations are locked in the shear links, which can be removed using a plasma cutter or an oxygen-acetylene torch. For the installation of the second set of shear links, that was used on the LCF-3L specimen, bottom bolts of one of the linked columns attached to the base plate had to be loosened up. The actuator side was selected for this operation. The shear links were removed in the following order: 1) link at mid-height of second floor, 2) link at mid-height of first floor, 3) link at second level floor, 4) link at first level floor, and 5) link at foundation level. Removing deformed shear links 
from the system and replacing them by new ones proved to be an easy task. The total amount of time spent in this activity was about 8 hours. Figure (6.22) shows the LCF-5L specimen when the link at mid-height of second floor was removed, while Figure (6.23) shows all deformed shear links after the LCF-5L specimen was tested.

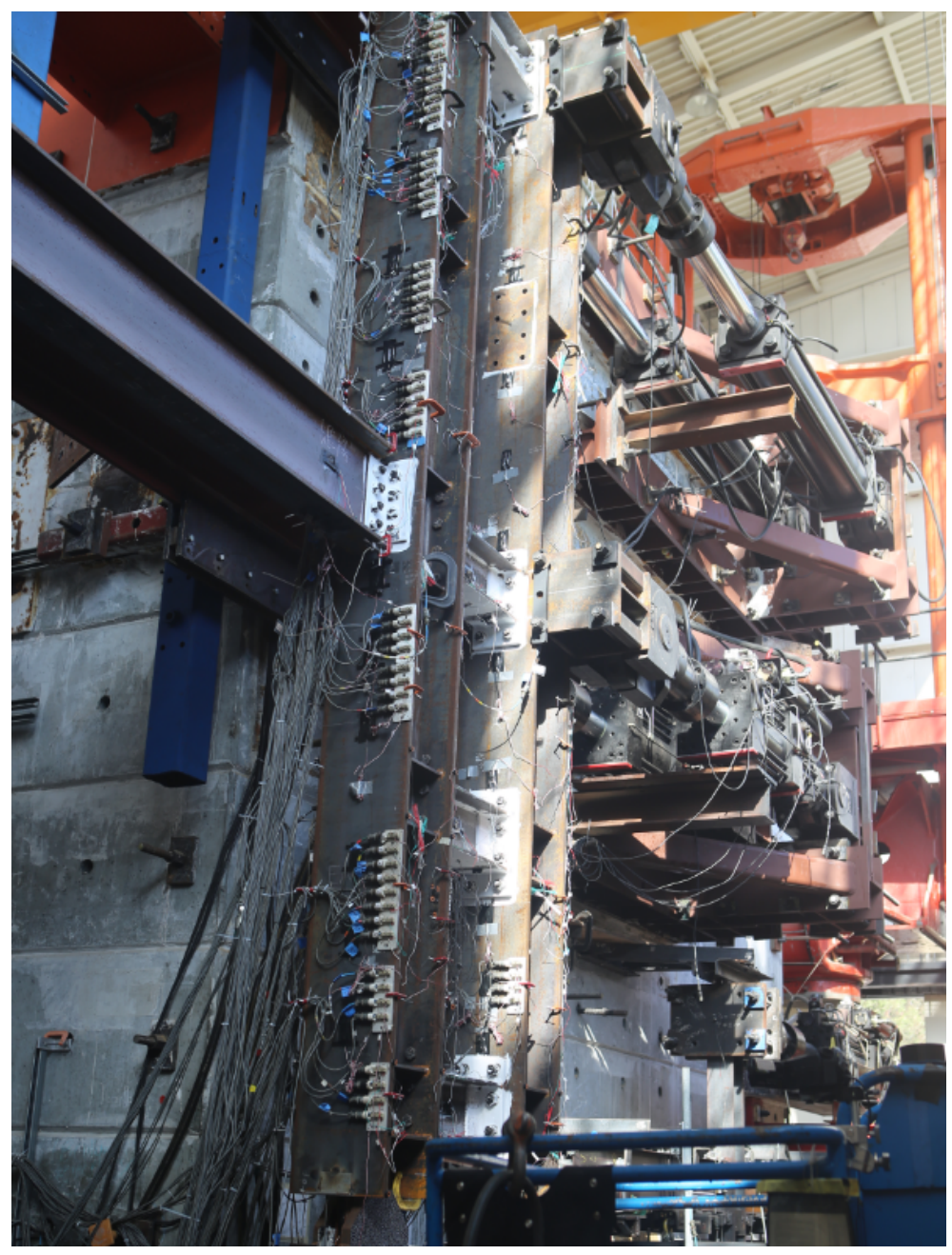

Figure 6.22: Replaceability of shear link at mid-height of second floor. 

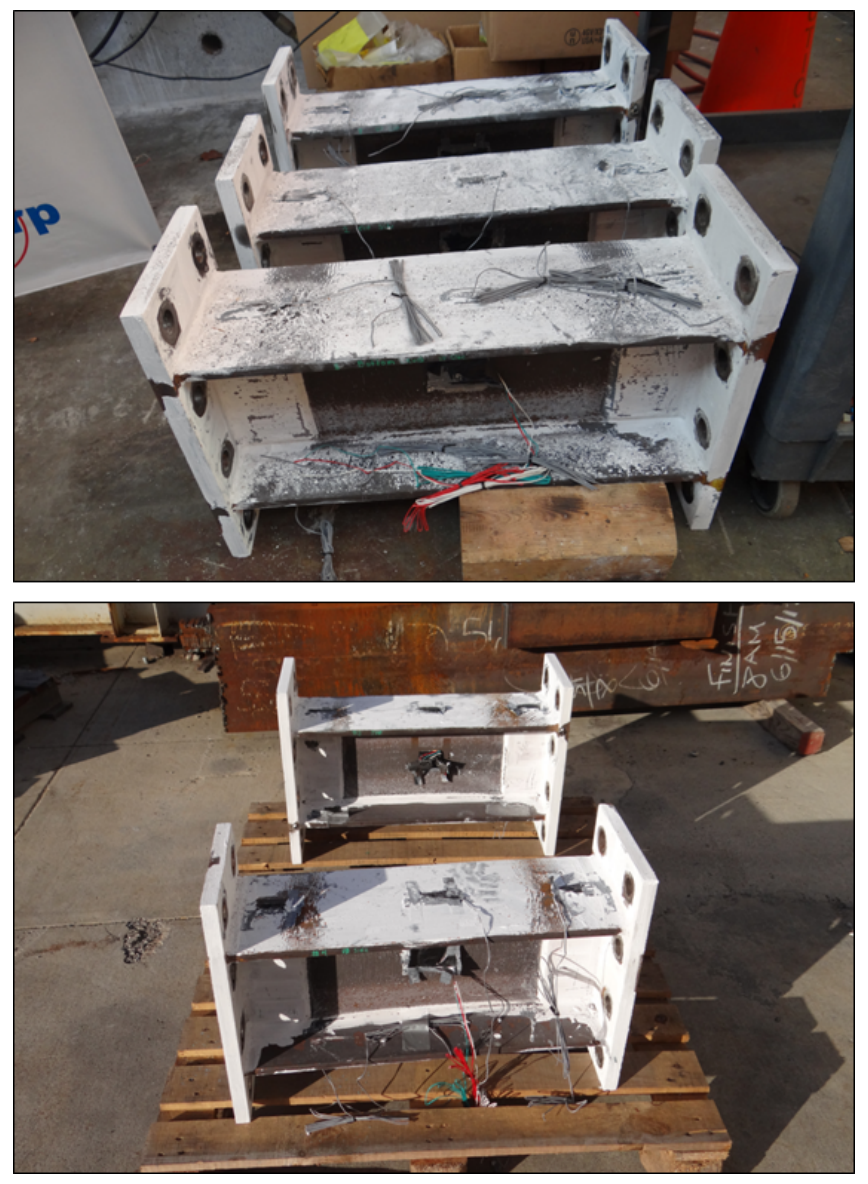

Figure 6.23: Deformed shear links after testing the LCF-5L specimen.

\subsection{Seismic Response of LCF-3L}

A 2-story, 1-bay physical specimen was built in the laboratory and a hybrid model was defined in a OpenSees tcl file. In this tcl file, two generic experimental elements, two rigid-link trusses and a leaning column were defined. Figure (6.24) shows the model used for the hybrid simulation. For the LCF-3L specimen, p-delta effects were considered through introducing a leaning column in the OpenSees model.

For the hybrid simulation testing on the LCF-3L specimen, a combined 


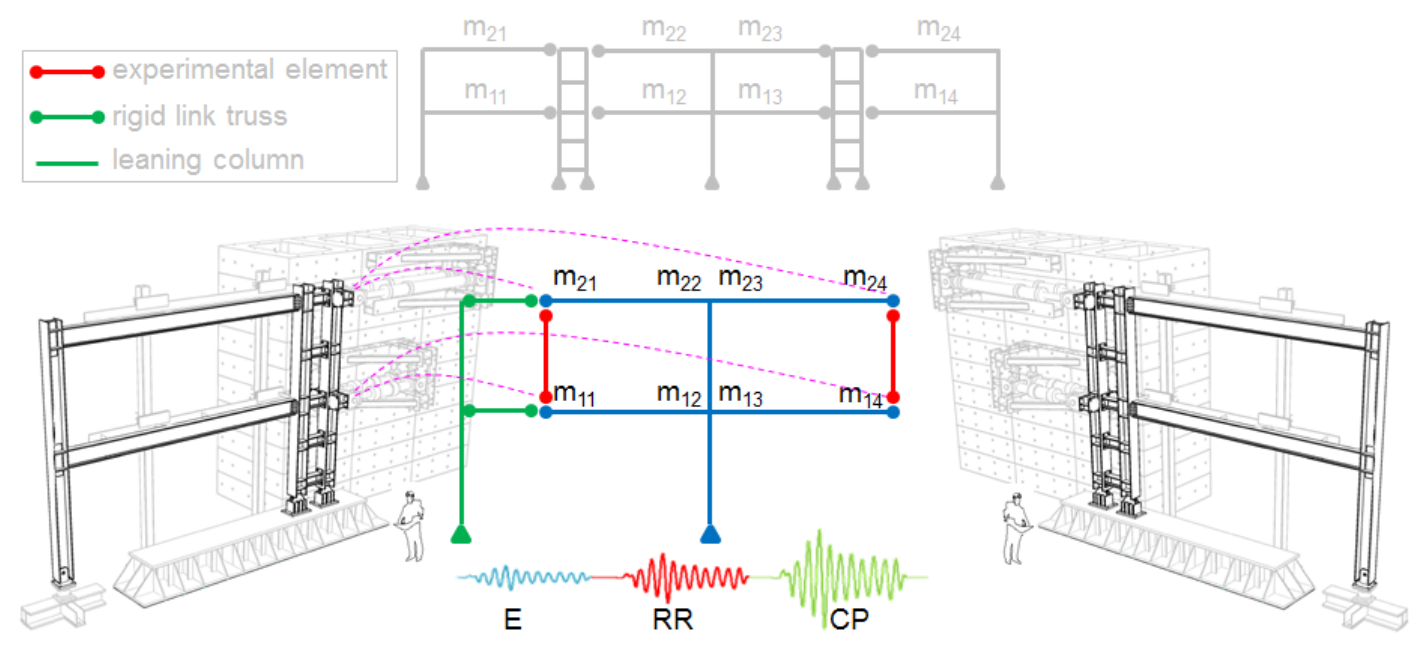

Figure 6.24: fig:LCF hybrid model.

ground motion input time history was used as well. A total of five earthquakes (EE, RR-1, RR-2, CP-1, and CP-2) were used on the LCF-3L specimen. The test was conducted very slowly compared to real time and continued until the end of the last earthquake. The experimental earthquake response is presented in terms of total base shear versus drift. The application of the ground motions revealed a sequence of yielding events as follows: Link A at base, Link B at first story, Link $\mathrm{C}$ at second story, GB at first story, and GB at second story. Figure (6.25) shows that the structure displaced up to $1.8 \%$ drift and $2.8 \%$ when subjected to earthquakes RR1 \& RR2 and CP1 \& CP2, respectively.

Following the same approach as discussed in Section (6.3), figures (6.26) and (6.27) show the seismic internal axial loads on LCs along its height as well as the Seismic Provisions and LCF design values for comparison. Experimental results indicate similar behavior on both columns and maximum values that 

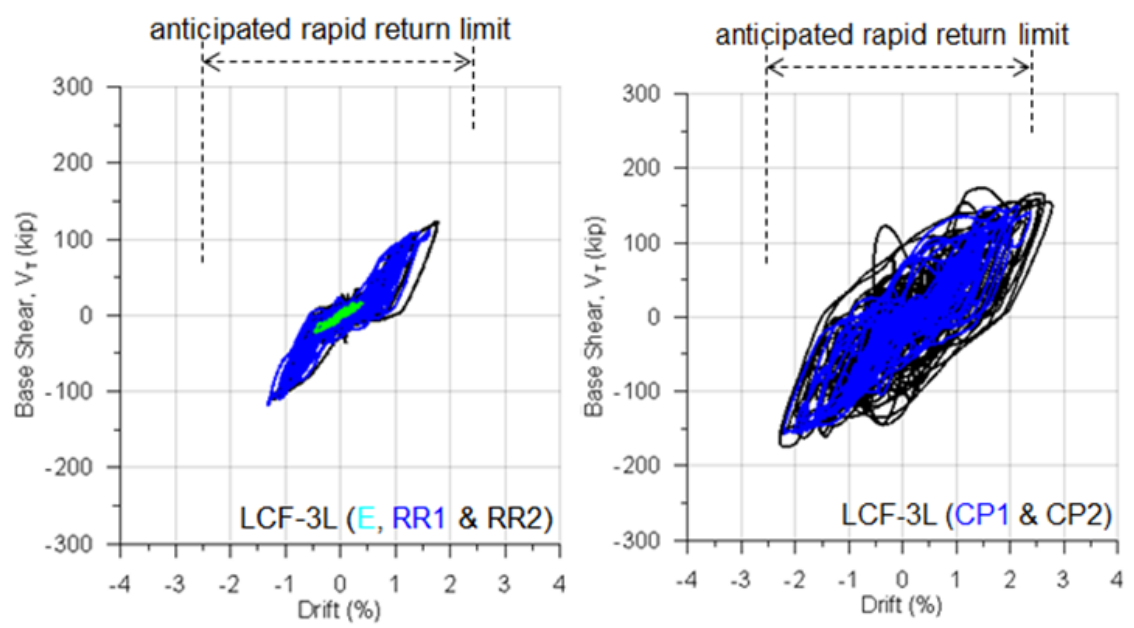

Figure 6.25: Base shear versus drift.

corroborate with the overstrength LCF design factor of 2.0.

Figure (6.26) shows a relatively well distributed axial load along the column and a maximum axial load on the foundation of about 720 kips. As expected, the increase of axial loads was obtained while changing the level of earthquake. Using Seismic provisions overstrength factor had shown that the structure might be underdesigned if this factor is used to design the foundation element. A similar behavior was obtained in Figure (6.27) with a maximum axial load on the foundation of also about 720 kips. Even though an overstrength factor of 2.0 was used for the LCF design, a difference of about $18 \%$ was noted between the LCF design value and the maximum axial load on the linked columns, suggesting that a overstrength factor greater than 2.0 might be considered in the future, while considering the collapse prevention performance level.

In addition to the analyses of axial loads on the linked columns, an evaluation of shear forces on the replaceable links was performed. Once the axial loads were 


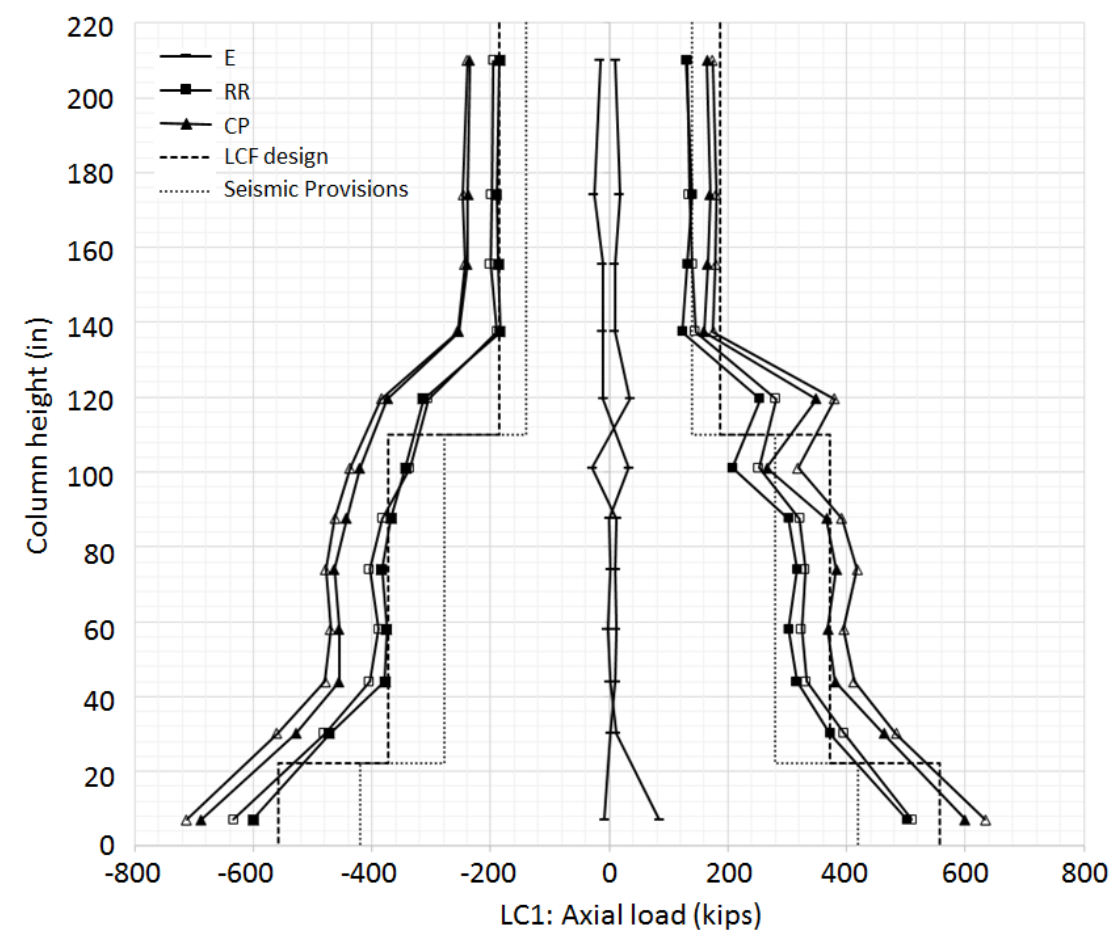

Figure 6.26: Axial loads along LC1 height for LCF-3L specimen

determined through strain gage data, the next step was to calculate the shear on the replaceable links. This procedure was done taking the difference on axial loads between the column above and below the replaceable link. For example, the shear on Link A for the collapse prevention level was obtained from the difference between 550 kips and 355 kips which corresponds to 195 kips. In order to capture the correct shear on the link located at the floor levels, Links $\mathrm{B}$ and $\mathrm{C}$, the shear from the gravity beam must be calculated as well. Figure (6.19) shows a free-body diagram to calculate shear on links. For example, the shear on Link B for the rapid return to occupancy level was obtained from the difference between 326 kips and 150 kips which corresponds to 176 kips. 


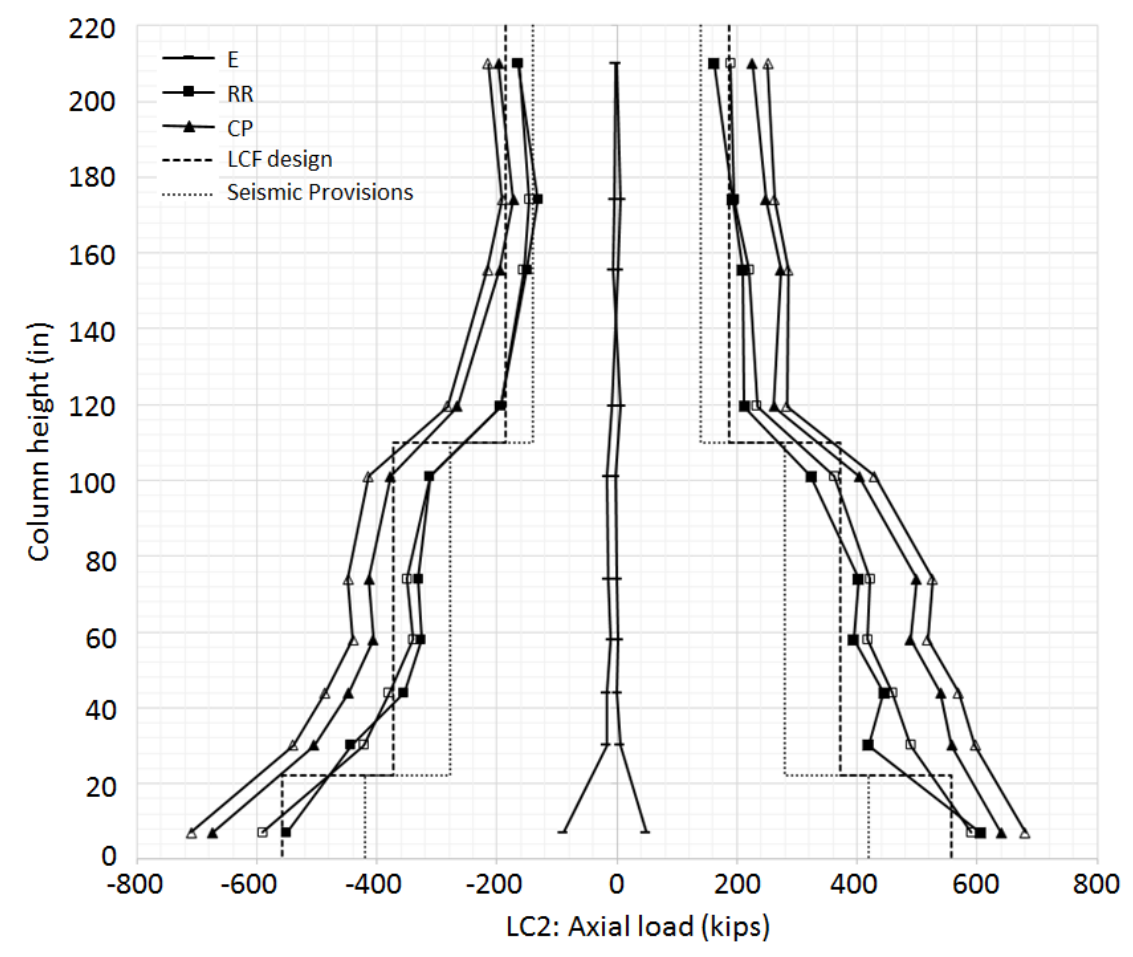

Figure 6.27: Axial loads along LC2 height for LCF-3L specimen

This value needs to be subtracted from the gravity beam shear which is 4 kips. Therefore, shear on Link B is 172 kips. Figure (6.28) summarizes the shear force on links for each level of earthquake.

Figures (6.29) and (6.30) show link rotation for all rapid return to occupancy and collapse prevention performance levels, respectively. The link rotation demands in Link A at base are greater than in Link $\mathrm{C}$ at second story which concur with the sequence of yielding events mentioned above. Whitewash on the links began flaking near mid-span, then propagated toward the end plates as shown in Figure (6.31). In the collapse prevention performance level the links have larger inelastic demand and are more likely to require replacement. It should 


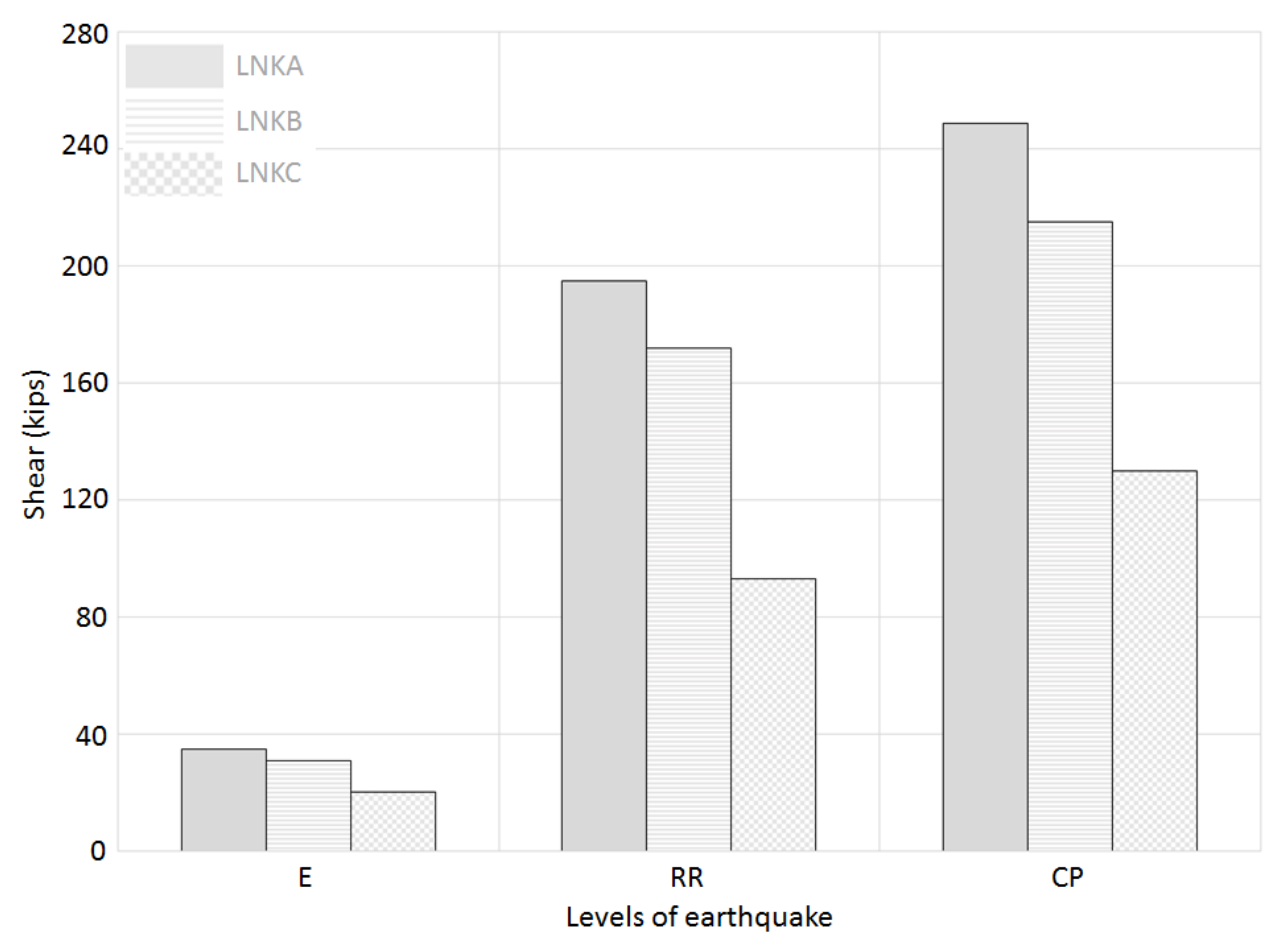

Figure 6.28: Shear on links vs levels of earthquake for LCF-3L specimen.

be noted that none of the links failed under any ground motions used in the hybrid simulation.

Figure (6.32) shows the gravity beam flange strain distribution for the rapid return to occupancy and collapse prevention performance levels. The yielding strain $\epsilon_{y}$ is shown by horizontal dashed lines for reference and was obtained using Hooke's law. As shown, beam flange yielding does not occur until collapse prevention performance level is achieved. This ensures that no repairs would be necessary and will help to minimize post event repair costs. Figure (6.32) also shows limited demands on gravity beams and this could indicate less rigorous detailing connections. LCF-3L specimen exhibited three regions within the lateral response; elastic, yielding of links and yielding of links as well as MF 

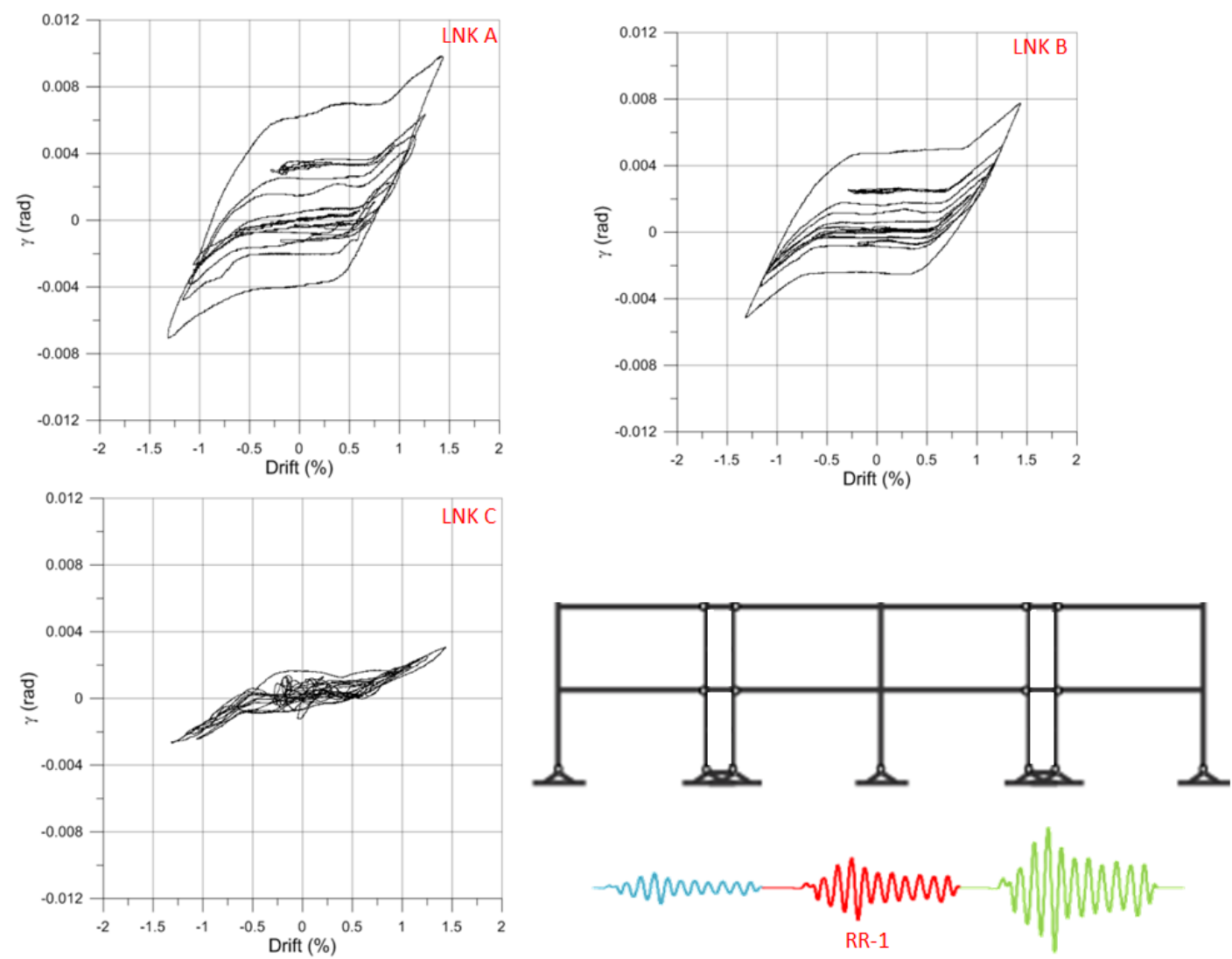

Figure 6.29: Shear deformation versus drift for LCF-3L specimen during RR earthquake.

beams. Provided the links are replaceable, these correspond to three distinct performance levels; elastic, rapid return to occupancy and collapse prevention. No significant permanent drift was observed during the earthquake motions.

\subsection{Ultimate Cyclic Loading}

In the framework of the project two full scale experimental specimens could be tested. The LCF is a new lateral system and has never been tested before, hence after the hybrid test was finished; the physical LCF sub-system was tested under 

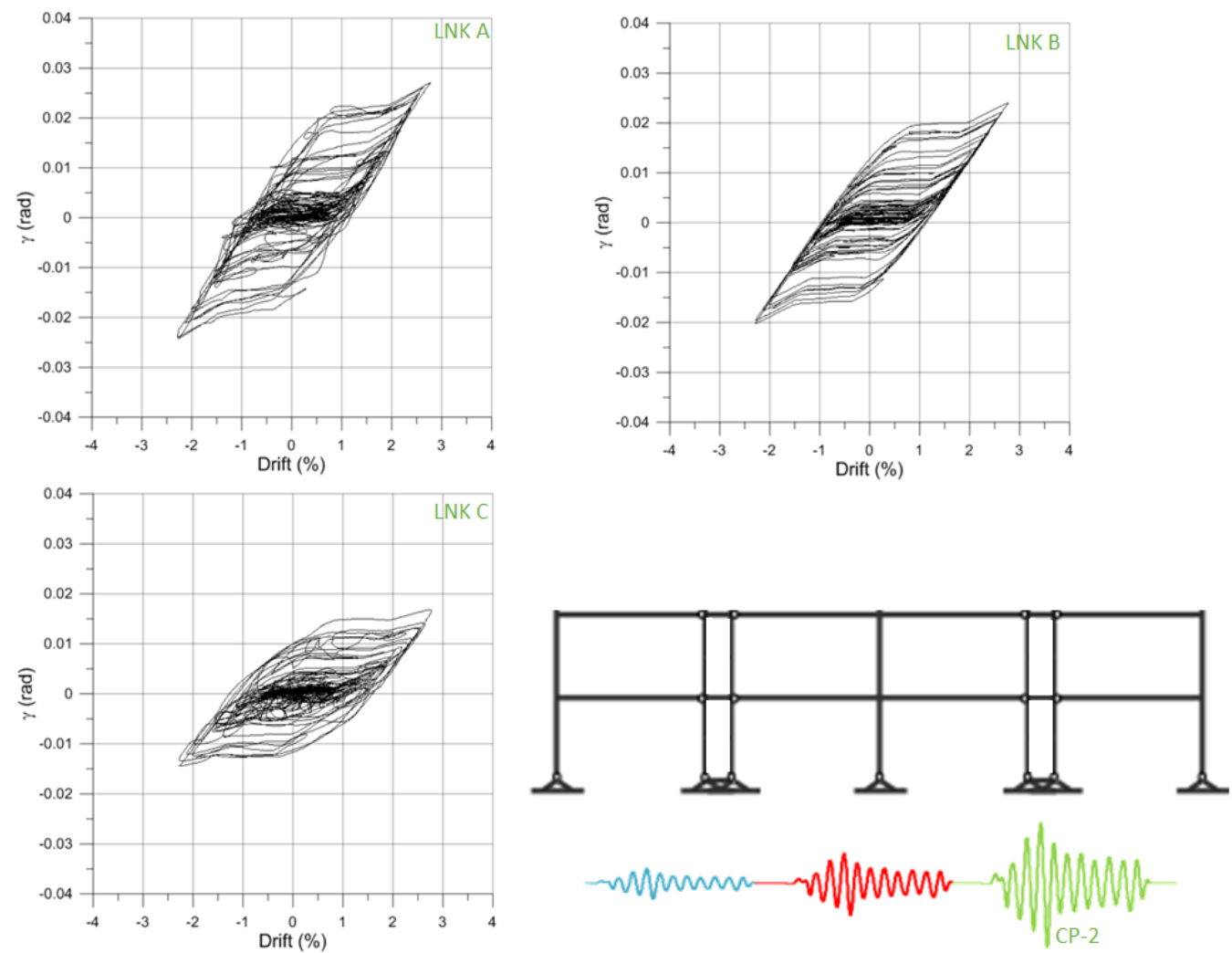

Figure 6.30: Shear deformation versus drift for LCF-3L specimen during CP earthquake.

an ultimate cyclic loading up to $5 \%$ total drift. Lateral loads were applied at first and second story elevations using four servo-controlled hydraulic actuators, two per floor. The displacements of both first and second story were monitored and controlled during the entire test. Figure (6.33) shows the maximum response in terms of hysteresis loop of the physical LCF sub-system. A maximum second story displacement of 10.8 in. with a 200 kip base shear was obtained. In regards to the shear links, Figure (6.33) also shows the link rotation demands in Link $\mathrm{A}(\gamma=0.07 \mathrm{rad}$.$) are greater than in Link \mathrm{C}(\gamma=0.05$ rad. $)$. Web buckling did not start to form in the web of the shear link until $4 \%$ drift. With 

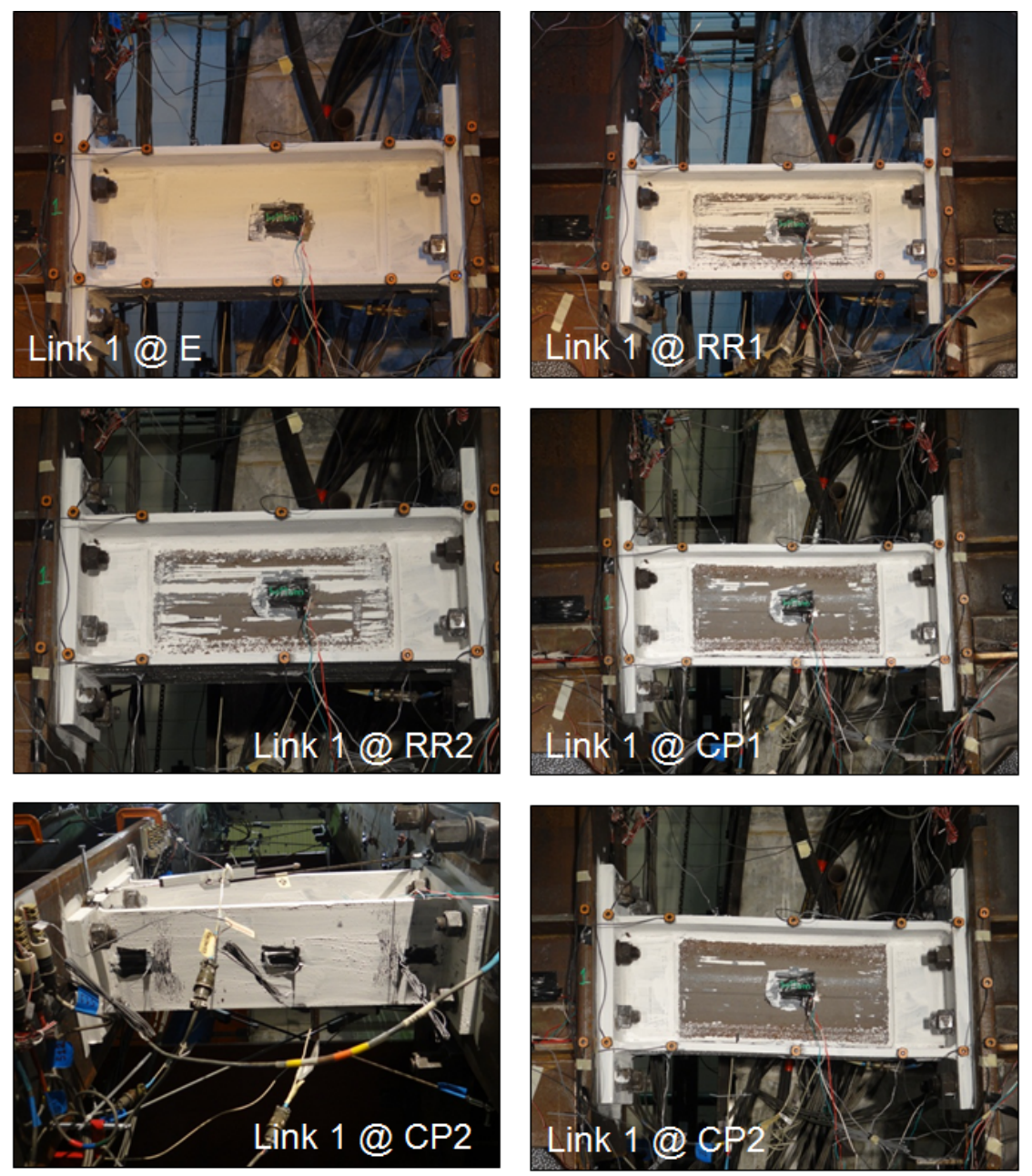

Figure 6.31: Progression of damage of Link A for LCF-3L specimen.

the progression of the cycles, web buckling became more pronounced and a crack started at mid-span followed by a crack between the top flange and web of the link. The cracks kept propagating until 5\% drift. The web began to tear and the test was stopped. Figure (6.34) shows the behavior of Links A, B and C; and gravity beams 1 and 2 at 5\% drift. Shear links shown to be effective in protecting gravity system and participating well past $4 \%$ drift and gravity beams had limited damaged. The friction test apparatus used after the ultimate 

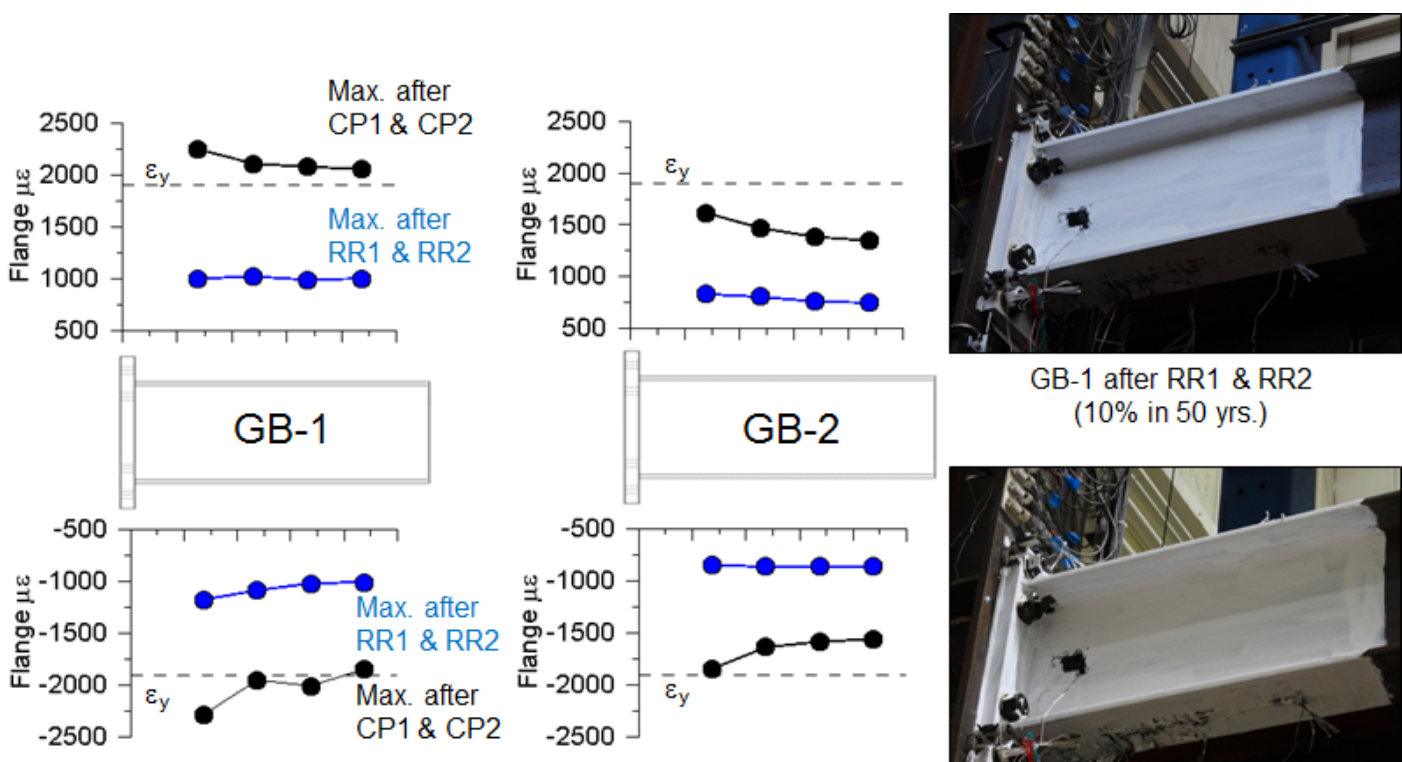

GB-1 after RR1 \& RR2 $(10 \%$ in $50 \mathrm{yrs}$.)

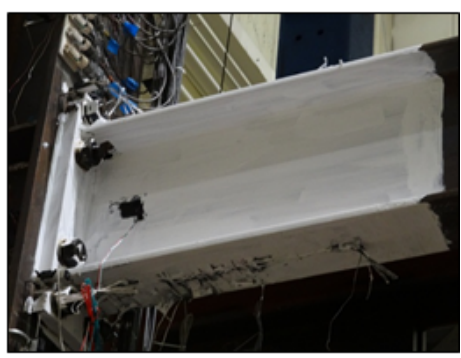

GB-2 after CP1 and CP2 ( $2 \%$ in 50 yrs.)

Figure 6.32: Flange strain distribution of gravity beams for LCF-3L specimen.

cyclic loading test is shown in Figure (6.35).

All in all, the LCF system exhibited three distinct performance levels; linearly elastic, rapid return to occupancy where only the replaceable links would yield, and collapse prevention where the gravity beam components also became damaged. From visual inspection, whitewash on the web links began flaking near midspan, then propagated toward the end plates. Links yielded prior gravity beams, all columns remained elastic, and the ultimate system failure mode was ductile and non-catastrophic. The LCF system is a viable system under cyclic and seismic loading, offering a ductile structural system with the ability to rapidly return to occupancy. 

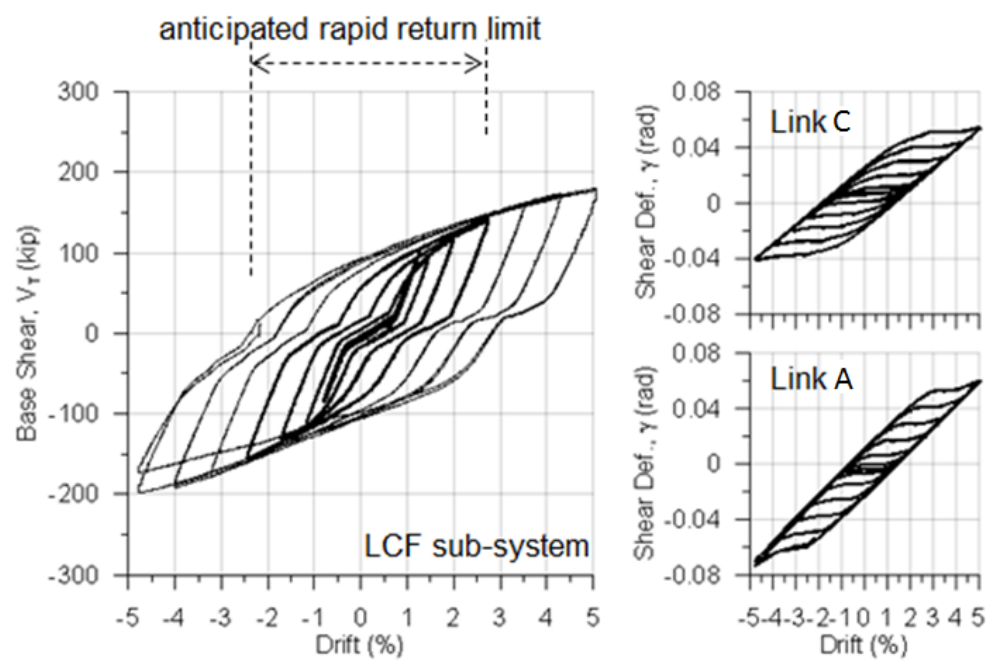

Figure 6.33: Base shear versus drift; Link shear deformation versus drift hysteresis.
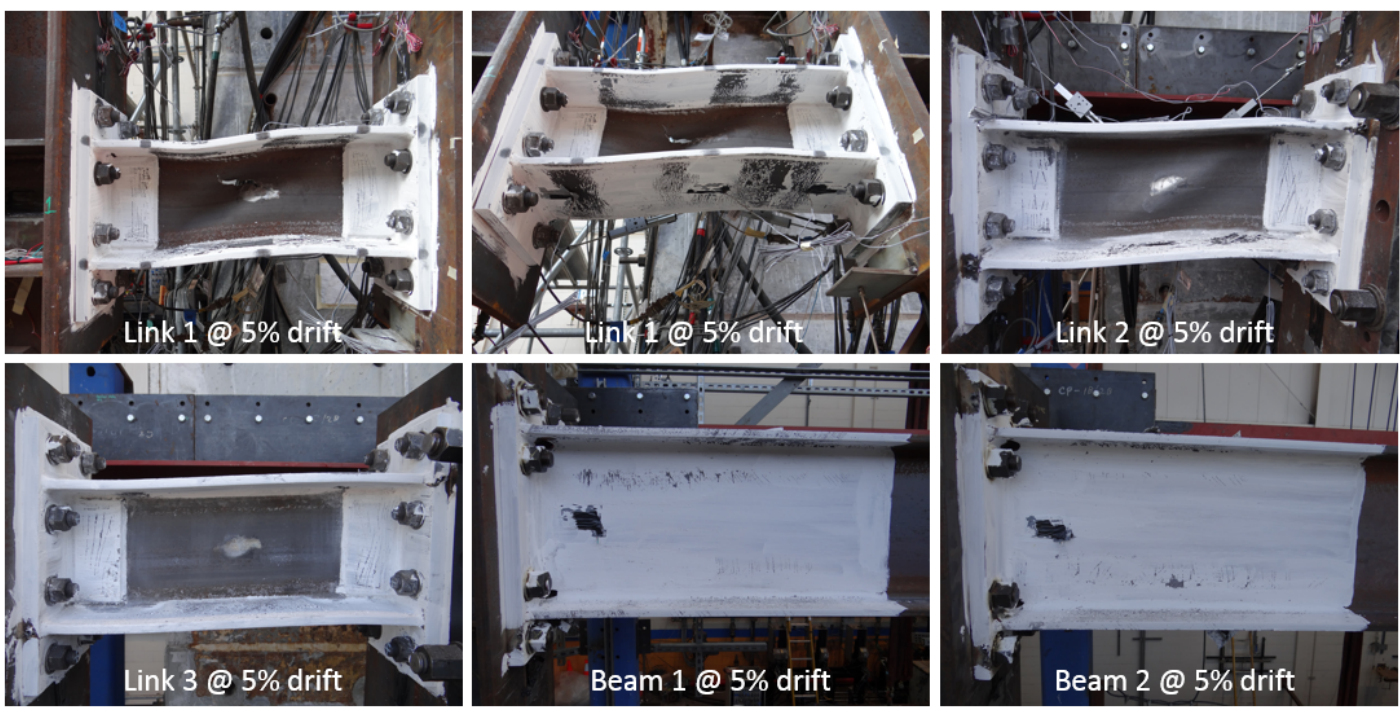

Figure 6.34: Damage in structural members at 5\% drift. 


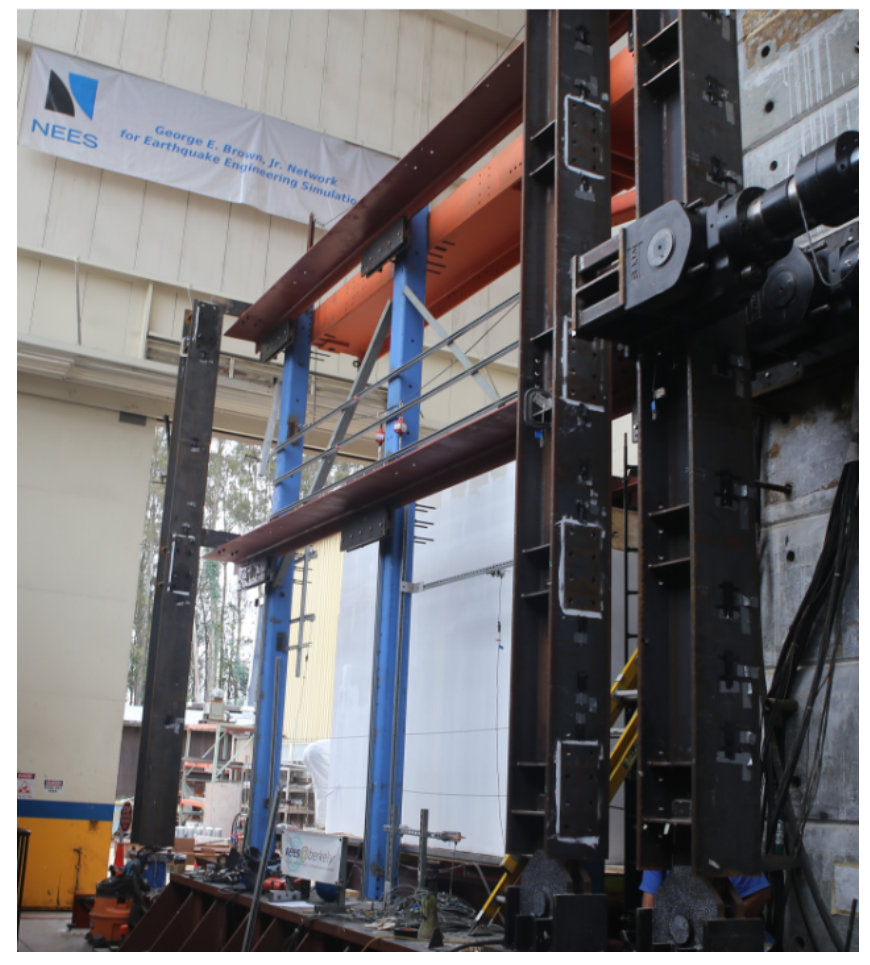

Figure 6.35: Friction test setup. 


\section{Chapter 7}

\section{LCF Structural Mechanics}

\subsection{Introduction}

An analytical investigation was performed on the LCF system and verified based on results from rigorous iterative design conducted for experimental frame. This investigation is presented based on the mechanics of the system to evaluate the lateral stiffness of LCF buildings in order to develop closed-form equations. The expressions developed may be used to select the geometric properties not only for the closely spaced dual columns but also the shear links, meeting the design inter-story drift limits. Analytically estimating the stiffness provides the designers with tools to size structural members and provide a first estimate of drift under earthquake loading, which is useful since the LCF system tends to be drift controlled.

One of the main issues in regards to the LCF design is how to conduct an efficient and accurate preliminary analysis based on elementary structural 
mechanics and select optimum member sizes to be used in the eventual computer analysis portion of the design. This chapter addresses consistent and accurate approaches for member proportioning of two-dimensional LCF multistory frames subjected to lateral loads. For the stiffness estimation, the first approach, denoted hereafter as cantilever column, is based on the assumption that the linked columns of the LCF building could be represented by a cantilever column and is used to determine the member sizing of the linked columns. The second approach, denoted hereafter as Vierendeel column, is based on the assumption that the linked columns of the LCF building could be represented by a rectangular configuration with rigid joints. This latter approach is used to determine the member sizing of the links. In addition to estimating the lateral stiffness, closed-form equations were developed to size gravity beam members as well to estimate the axial load on the foundation element using parameters already known by practitioners.

\subsection{Cantilever Column}

In order to achieve more predictable structural performance under lateral seismic forces, knowledge of the ultimate structural behavior of the structure is essential. Therefore, design factors such as drift for given hazard levels should become part of the design process from the beginning. The important factors affecting the behavior of LCF buildings using the cantilever column are: allowable story drift, story height, lateral seismic force given by the equivalent lateral 
force procedure, and modulus of elasticity.

Consider, for example, a 2-story LCF building subjected to lateral seismic forces represented by a cantilever column shown in Figure (7.1). The lateral deflection of the 2-story LCF building is given by Equation (7.1):

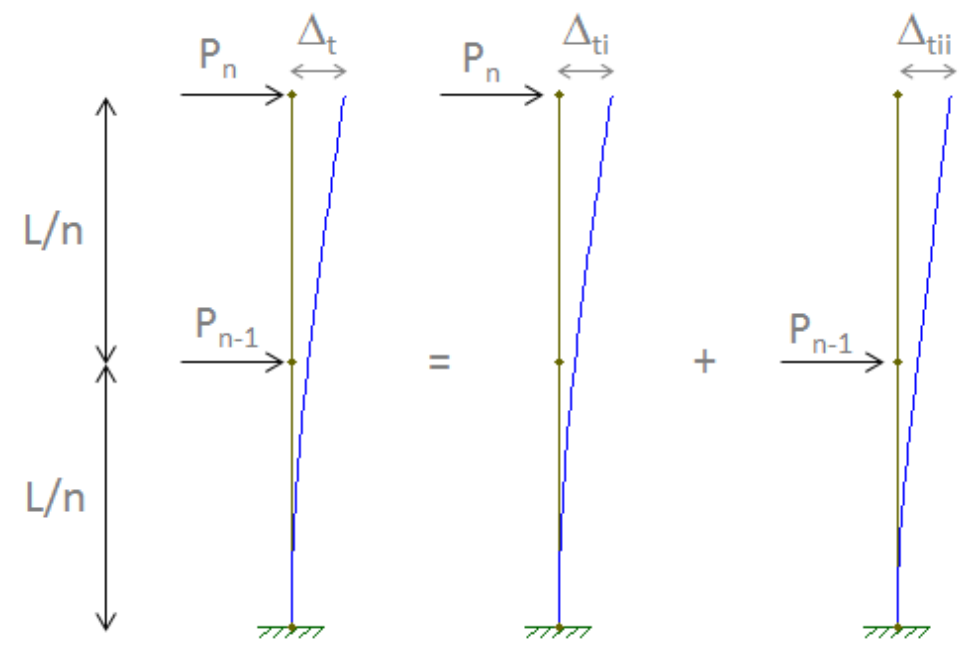

(i)

(ii)

Figure 7.1: Cantilever column of a 2-story LCF building.

$$
\Delta_{t}=\Delta_{t_{i}}+\Delta_{t_{i i}}
$$

in which $L$ is the total height, $n$ is the story number, $\Delta_{t}$ is the total lateral displacement at the second story, $\Delta_{t_{i}}$ is the lateral deflection caused by a seismic force at the second story, and $\Delta_{t_{i i}}$ is the lateral deflection caused by a seismic force at the first story. The total lateral displacement and considering flexure only is obtained the equation of virtual work given by Equation (7.2): 


$$
\Delta_{t}=\int_{0}^{L} \frac{m M}{E I} d x
$$

where $M$ is the internal moment caused by the real load, $m$ is the internal moment caused by the external virtual unit load, $E$ is the modulus of elasticity, and $I$ is the moment of inertia. An external virtual unit load is applied to the structure at the point and in the direction of the desired displacement. With the virtual load placed and all the real loads removed from the structure, the internal virtual moment is calculated. Using the same coordinates as those established for the internal virtual moment, the internal moment caused only by the real loads is determined. Therefore, for a 2 -story LCF building, $\Delta_{t_{i}}$ and $\Delta_{t_{i i}}$ are given by the following equations:

$$
\begin{gathered}
\Delta_{t_{i}}=\frac{P_{2} L^{3}}{3 E I} \\
\Delta_{t_{i i}}=\frac{5 P_{1} L^{3}}{48 E I}
\end{gathered}
$$

Substituting the equations above in Equation (7.1) and applying the limits such as $P_{2}=2 P$ and $P_{1}=P$, the total lateral deflection at the second story of the LCF building is given by Equation (7.5):

$$
\Delta_{t}=\frac{37 P L^{3}}{48 E I}
$$


The only unknown of Equation (7.5) is the moment of inertia which can be used to determine what sections should be used in the linked columns.

Now consider a 3-story LCF building subjected to lateral seismic forces represented by a cantilever column shown in Figure (7.2). The lateral deflection of the 3-story LCF building is given by Equation (7.6):

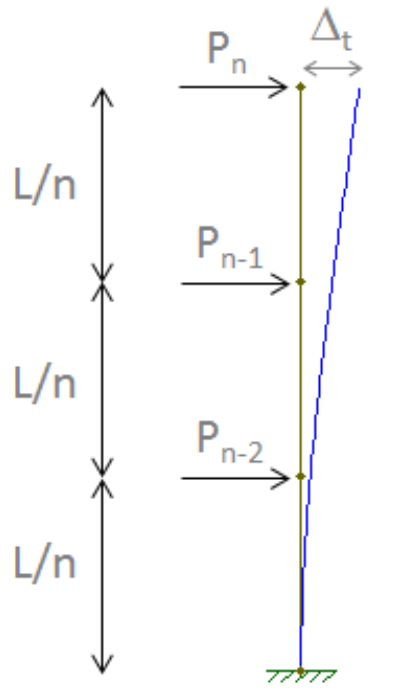

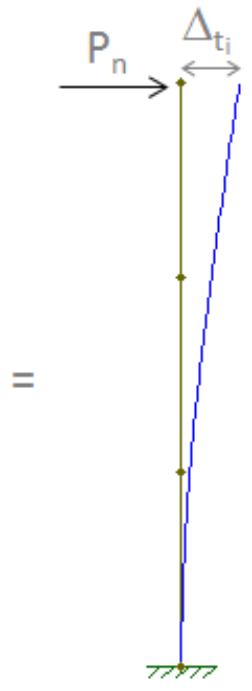

(i)

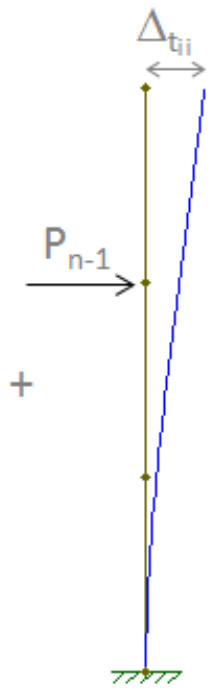

(ii)

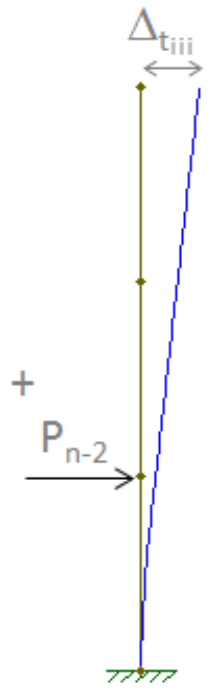

(iii)

Figure 7.2: Cantilever column of a 3-story LCF building.

$$
\Delta_{t}=\Delta_{t_{i}}+\Delta_{t_{i i}}+\Delta_{t_{i i i}}
$$

For a 3-story LCF building, $\Delta_{t_{i}}, \Delta_{t_{i i}}$ and $\Delta_{t_{i i i}}$ are given by the following equations:

$$
\Delta_{t_{i}}=\frac{P_{3} L^{3}}{3 E I}
$$


Table 7.1: Partial and total displacements for different story levels.

\begin{tabular}{|c|c|c|c|c|c|c|c|}
\hline $\mathrm{x}$ & $\Delta_{\mathrm{ti}}$ & $\Delta_{\mathrm{tii}}$ & $\Delta_{\mathrm{tiii}}$ & $\Delta_{\mathrm{tiv}}$ & $\Delta_{\mathrm{tv}}$ & $\Delta_{\mathrm{tvi}}$ & $\Delta_{\mathrm{t}}$ \\
\hline 2 & $\frac{P_{2} L^{3}}{3 E I}$ & $\frac{5 P_{1} L^{3}}{48 E I}$ & - & - & - & - & $\frac{37 P L^{3}}{48 E I}$ \\
\hline 3 & $\frac{P_{3} L^{3}}{3 E I}$ & $\frac{14 P_{2} L^{3}}{81 E I}$ & $\frac{12 P_{1} L^{3}}{243 E I}$ & - & - & - & $\frac{113 P L^{3}}{81 E I}$ \\
\hline 4 & $\frac{P_{4} L^{3}}{3 E I}$ & $\frac{P_{3} L^{3}}{5 E I}$ & $\frac{5 P_{2} L^{3}}{481 E I}$ & $\frac{3 P_{1} L^{3}}{100 E I}$ & - & - & $\frac{11 P L^{3}}{5 E I}$ \\
\hline 5 & $\frac{P_{5} L^{3}}{3 E I}$ & $\frac{23 P_{4} L^{3}}{100 E I}$ & $\frac{7 P_{3} L^{3}}{50 E I}$ & $\frac{3 P_{2} L^{3}}{25 E I}$ & $\frac{P_{1} L^{3}}{50 E I}$ & - & $\frac{33 P L^{3}}{10 E I}$ \\
\hline 6 & $\frac{P_{6} L^{3}}{3 E I}$ & $\frac{P_{5} L^{3}}{4 E I}$ & $\frac{14 P_{4} L^{3}}{81 E I}$ & $\frac{5 P_{3} L^{3}}{48 E I}$ & $\frac{12 P_{2} L^{3}}{243 E I}$ & $\frac{17 P_{1} L^{3}}{1296 E I}$ & $\frac{437 P L^{3}}{100 E I}$ \\
\hline
\end{tabular}

$$
\begin{gathered}
\Delta_{t_{i i}}=\frac{14 P_{2} L^{3}}{81 E I} \\
\Delta_{t_{i i i}}=\frac{12 P_{1} L^{3}}{243 E I}
\end{gathered}
$$

Substituting Equations (7.7), (7.8) and (7.9) in Equation (7.6) and applying the limits such as $P_{3}=3 P, P_{2}=2 P$ and $P_{1}=P$, the total lateral deflection at the third story of the LCF building is given by Equation (7.10).

$$
\Delta_{t}=\frac{113 P L^{3}}{81 E I}
$$

Similar expressions for partial and total deflections can be obtained for different story levels. Lopes et. al. $\left(2012^{a}\right)$ and Malakoutian et. al. (2013) numerically analyzed 6-story LCF buildings using pushover and time-history analyses, respectively. For completeness, the cantilever column approach is summarized in Table (7.1) up to 6-story buildings as illustrated in Figure (7.3). 


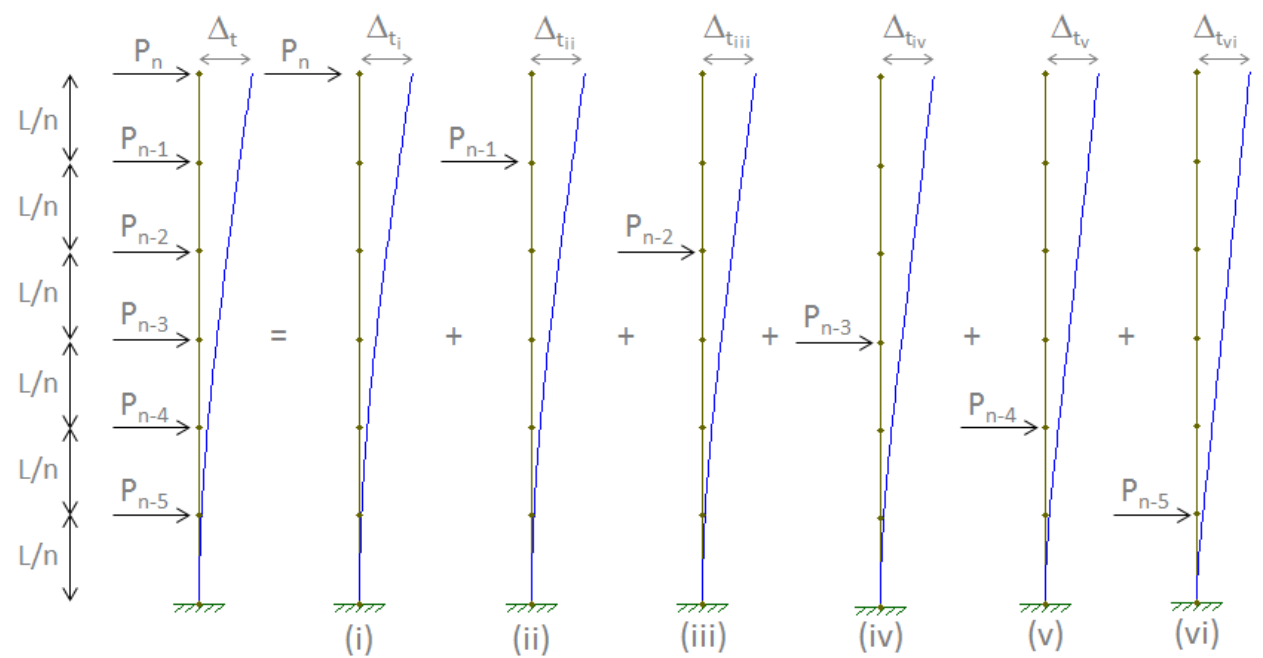

Figure 7.3: Cantilever column of a 6-story LCF building.

Total displacement parameters have been plotted in Figure (7.4) for a varying number of stories. A power trendline was used to obtain a recurrence equation, given by Equation (7.11), for the moment of inertia of the Linked Column.

$$
I_{L C}=\frac{x^{4.6} P L^{3}}{8 E \Delta}
$$

where $I_{L C}$ is the moment of inertia for a single linked column, $x$ is the number of stories and $h$ is the story height of the LCF building. Note that the linked columns are also known as dual columns, hence the moment of inertia $\left(I_{L C}\right)$ is already divided by 2 in order to obtain the member sizing for a single linked column. 


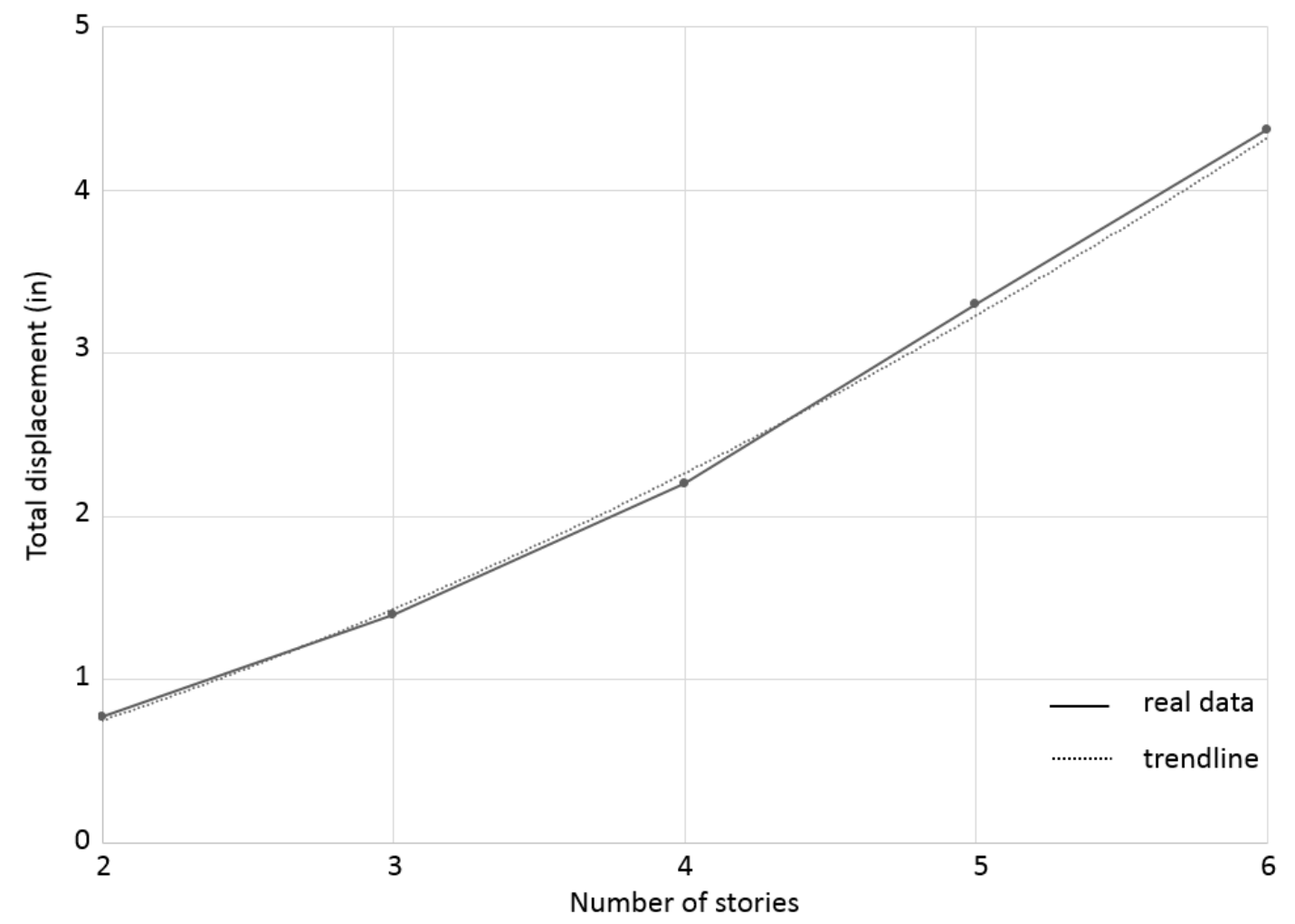

Figure 7.4: Total displacement versus number of stories.

\subsection{Vierendeel Column}

The Vierendeel column approach is based on the assumption that the linked columns of the LCF building could be represented by a rectangular configuration with rigid joints. Vierendeel systems take advantage of the member's resistances eliminating the need for diagonal members. Figure (7.5) shows a LCF Vierendeel column where $h$ is the distance between links, $H$ is the length of the link and $I_{L}$ is the link's moment of inertia, which is the unknown. Assume nodes 1 and 4 are fixed and that nodes 2 and 3 move laterally, $\Delta$, the same amount.

Equations (7.12) give the moments at ends $i$ and $j$ of a member $i j$ in terms of 
joint rotations and translations at the ends of the member and also the fixed-end moments (FEM). These equations are known as the slope-deflection equations.

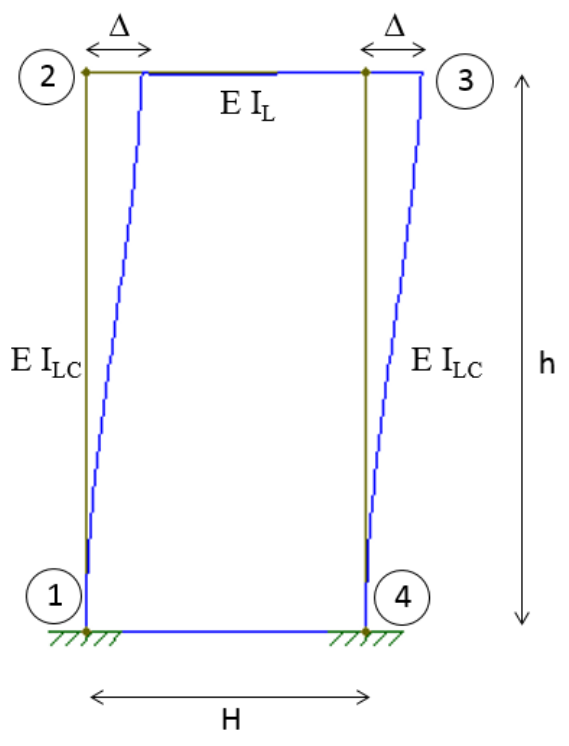

Figure 7.5: Vierendeel column.

$$
\begin{aligned}
& M_{i j}=M_{i j}^{F E M}+\frac{2 E I}{L}\left(2 \theta_{i}+\theta_{j}-3 \frac{\Delta}{L}\right) \\
& M_{j i}=M_{j i}^{F E M}+\frac{2 E I}{L}\left(\theta_{i}+2 \theta_{j}-3 \frac{\Delta}{L}\right)
\end{aligned}
$$

Using the slope-deflection equations, the end-moments for the Vierendeel column can conveniently be expressed as the following set of Equations (7.13): 


$$
\begin{gathered}
M_{12}=0+\frac{2 E I_{L C}}{h}\left(2 \theta_{1}+\theta_{2}-3 \frac{\Delta}{h}\right) \\
M_{21}=0+\frac{2 E I_{L C}}{h}\left(\theta_{1}+2 \theta_{2}-3 \frac{\Delta}{h}\right) \\
M_{43}=0+\frac{2 E I_{L C}}{h}\left(2 \theta_{4}+2 \theta_{4}-3 \frac{\Delta}{h}\right) \\
M_{34}=0+\frac{2 E I_{L C}}{h}\left(\theta_{4}+2 \theta_{3}-3 \frac{\Delta}{h}\right) \\
M_{23}=0+\frac{2 E I_{L C}}{H}\left(2 \theta_{2}+\theta_{3}\right) \\
M_{32}=0+\frac{2 E I_{L C}}{h}\left(\theta_{2}+2 \theta_{3}\right)
\end{gathered}
$$

Joint rotations at nodes 2 and 3 in Figure (7.5) are evaluated by writing equations of moment equilibrium at the joints that are free to rotate. This leads to the following set of Equations (7.14), known as compatibility equations.

$$
\begin{aligned}
& M_{21}+M_{23}=0 \\
& M_{32}+M_{34}=0
\end{aligned}
$$

Substitute Equations (7.13) in Equations (7.14) and assume $\theta_{2}=\theta_{3}=\theta$. Thus, 


$$
\left(\frac{3 E I_{L}}{H}+2 E I_{L C} h\right) \theta=\left(\frac{3 E I_{L}}{h^{2}}\right) \Delta
$$

ANSI/AISC 341 (2010) specifies the shear yielding links should be capable of developing a plastic rotation of $0.08 \mathrm{rad}$, whereas flexural yielding links should be capable of developing a plastic rotation of $0.02 \mathrm{rad}$. For a preliminary member sizing and using Equation (7.15), assume $\theta=0.02 \mathrm{rad}$ and also that the LCF should meet the design intent of $2.5 \%$ inter-story drift limits. Thus the moment of inertia of the link is accomplished by Equation (7.16).

$$
I_{L}=0.6 \frac{I_{L C} H}{h}
$$

\subsection{Serviceability}

In a design situation, it is common to develop approximate deflection equations in order to allow for quicker selection of a structural member based on deflection limitations. The gravity beams of the LCF system have got two different types of connections: extended end-plate moment connection and double-angle shear connection.

The indeterminate beam shown in Figure (7.6) models a LCF gravity beam and it is subjected to a uniformly distributed load. The load tends to deflect the beam as shown and the reactions are as follows: 


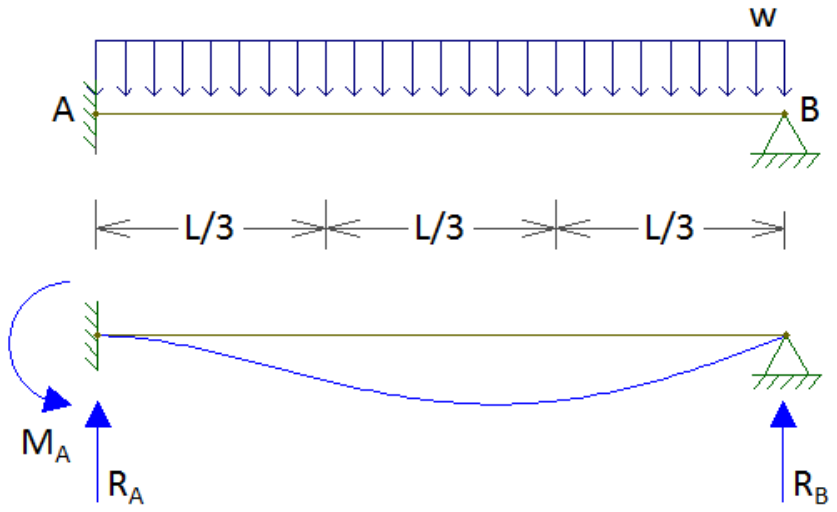

Figure 7.6: Gravity beam model for a LCF building.

$$
\begin{aligned}
R_{A} & =\frac{5 w L}{8} \\
R_{B} & =\frac{3 w L}{8} \\
M_{A} & =\frac{w L^{2}}{8}
\end{aligned}
$$

The bending moment equation, in function of $x$, from $B$ to $A$ in Figure (7.6) can be written as

$$
M(x)=\frac{3 w L}{8} x-\frac{w}{8} x^{2}
$$

where $x$ varies from 0 to $L$ and starts at support $B$.

Using the elastic curve equation and integrating twice, as well as applying the boundary conditions to find out the constants, the deflection equation of the beam shown in Figure (7.6) is given by Equation (7.19). 


$$
E I \Delta=\frac{w L}{16} x^{3}-\frac{w}{24} x^{2}+\frac{5 w L^{3}}{48} x
$$

Assume the maximum displacement occurs at $x=L / 3$ from support $B$. Therefore, Equation (7.19) can be rewritten as

$$
\Delta=\frac{71 w L^{4}}{1944 E I}
$$

Converting the units such that the moment is in kips - in, the beam length is in feet and, also, knowing that the two basic deflection limits are $L / 360$ and $L / 240$, Equation (7.20) can be modified such that the moment of inertia is calculated as follows:

$$
I_{G B}=\frac{4 w L^{3}}{5}
$$

where $I_{G B}$, in Equation (8.7), is the required moment of inertia of the gravity beam for $L / 360$ and $w$ is the live load.

Similarly, for the $L / 240$ case,

$$
I_{G B}=\frac{w L^{3}}{5}
$$

where $I_{G B}$, in Equation (7.22), is the required moment of inertia of the gravity beam for $L / 240$ and $w$ is summation of dead and live loads.

Equations (7.21) and (7.22) allow for quick selection of a shape based on a 
known load (dead and/or live) and beam span.

\subsection{Axial Load on Foundation}

One of the main goals in a preliminary analysis is to estimate the axial load at foundations. It should be said that the behavior of systems involving structurefoundation-soil interaction, especially when subjected to dynamic loads, is complex. The procedure used herein to determine the axial load on foundation is based upon statics.

Consider, for example, a 2-story LCF building subjected to lateral seismic forces represented by the linked columns shown in Figure (7.7). The lateral seismic force is obtained through the equivalent lateral force procedure and the total base shear is considered to be absorbed by the linked columns. First, determine the summation of moments about the center line of Figure (7.7) at the foundation level in order to calculate $R$. The axial load on the foundation element is given by Equation (7.23).

$$
R=\frac{5 P L}{2 H}
$$

where $R$ is axial foundation reaction for a single linked column, $P$ is the lateral seismic load, $L$ is the total height of the LCF building, and $H$ is the distance

between linked columns. Similar equations were obtained for 3-, 4-, 5- and 6-LCF buildings and results are summarized according to Table (7.2). 


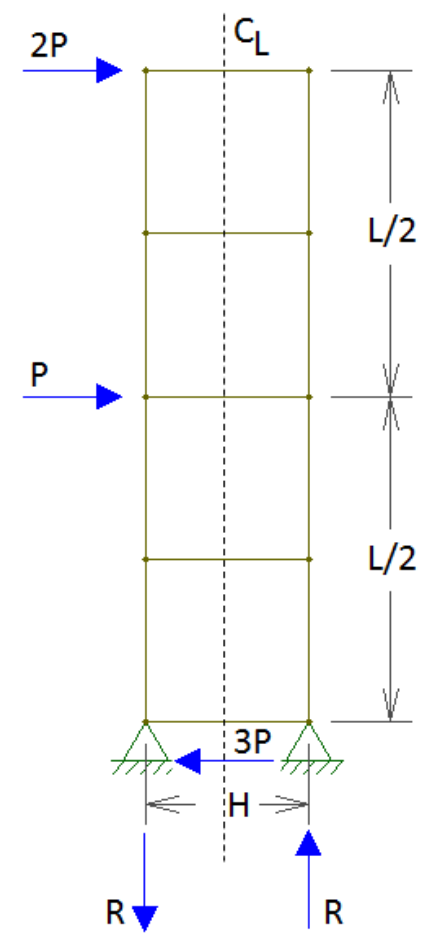

Figure 7.7: LCF 2-story linked column.

Foundation reaction parameters on Table (7.2) have been plotted in Figure (7.8) for a varying number of stories. A power trendline was used to obtain a recurrence equation, given by Equation (7.24), for the axial force on linked column foundations in function of the number of stories, $x$.

Table 7.2: Linked column axial force at foundation level.

\begin{tabular}{cc}
\hline $\mathrm{x}$ & $\mathrm{R}$ \\
\hline 2 & $\frac{5 P L}{2 H}$ \\
3 & $\frac{14 P L}{3 H}$ \\
4 & $\frac{15 P L}{2 H}$ \\
5 & $\frac{11 P L}{H}$ \\
6 & $\frac{91 P L}{6 H}$ \\
\hline
\end{tabular}




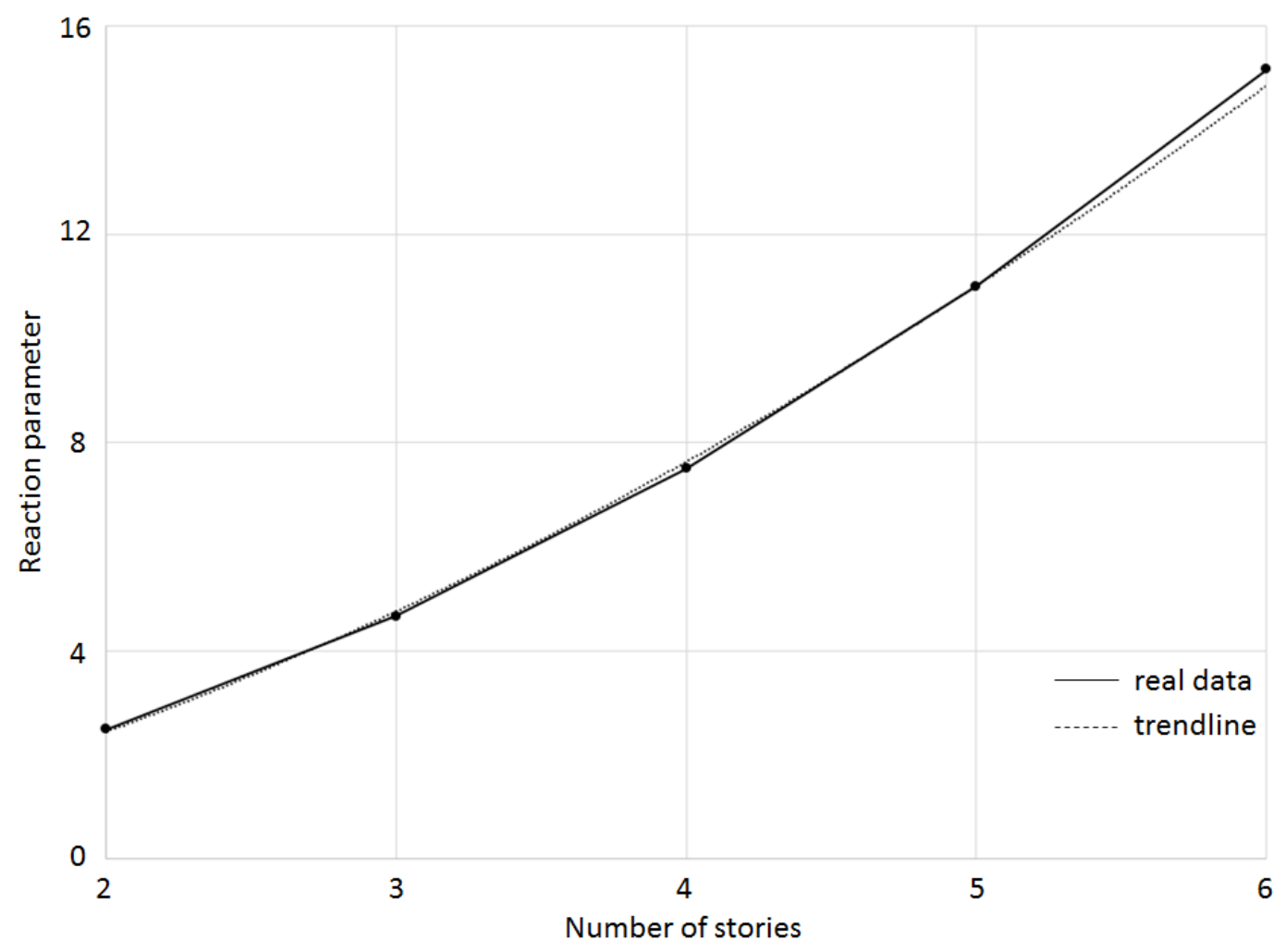

Figure 7.8: Foundation reaction parameters versus number of stories.

$$
R=\frac{17 x^{1.6} P L}{20 H}
$$

As a first example, consider both numerical and experimental results for a LCF 2-story building. Numerical investigation using pushover analysis indicated $R=509$ kips, whereas Equation (7.24), for $x=2, P=36$ kips, $L=220 \mathrm{in}$, and $H=42 \mathrm{in}$, provided $R=486$ kips. The difference is about $4.5 \%$. On the other hand, experimental results had shown a value of $R=957$ kips at $4 \%$ drift for the axial load on the foundation. Now, the difference is about $50 \%$.

This clearly indicates that an overstrength factor is necessary to realize the 
capacity design approach, which in this case is 2 . If the overstrength factor is too high, uneconomic structures will be designed, if it is too low, an undesirable mechanism may result. Based on the experimental results and the statics procedure presented in this Section, Equation (7.24) can be rewritten as:

$$
R=\Omega \frac{17 x^{1.6} P L}{20 H}
$$

Alternatively, consider that all shear links already achieved their plastic shear capacity, $V_{p}$, as shown in Figure (7.9). For the 2-story LCF building experimentally tested, there were five $\mathrm{W} 10 \mathrm{x} 45$ shear links with $V_{p}=93$ kips. Thus, the reaction at foundation level could also be estimated by Equation (7.26), where $\Omega$ is the foundation overstrength factor and $n$ is the number of shear links on the linked column. For this case, $R=930$ kips, with a difference about $4 \%$ when compared to the value obtained using Equation (7.25), $R=$ 972 kips.

$$
R=\Omega n V_{p}
$$

As a second example, consider the 3-story LCF building, LCF-W-L, presented in Chapter (4), there were seven W12x50 shear links with $V_{p}=87$ kips. For $x=3, P=25 \mathrm{kips}, L=351.5 \mathrm{in}$, and $H=60 \mathrm{in}$, Equation (7.25) gives a value for the reaction at foundation level as $R=1,212$ kips, whereas (7.26) indicates $R=1,218$ kips, a difference less than $1 \%$. 


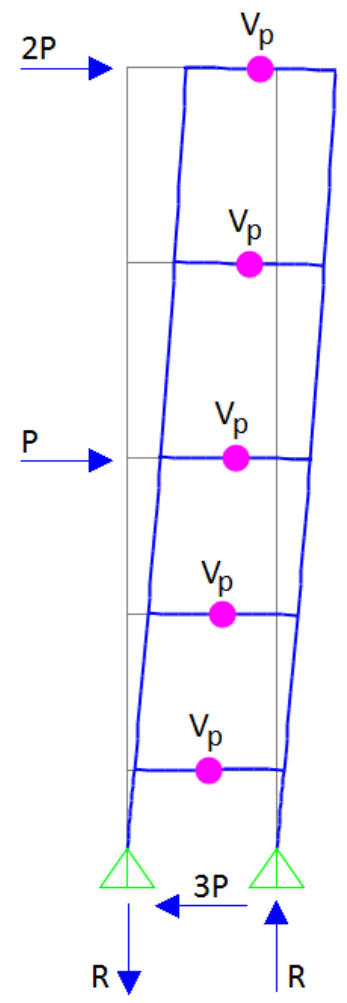

Figure 7.9: Plastic shear links on linked columns.

Finally, as a third example, consider a 4-story LCF building with the following design for shear links: three W14x145 with $V_{p}=272$ kips and two W10x45 with $V_{p}=98$ kips. For $x=4, P=13$ kips, $L=624 \mathrm{in}$, and $H=60 \mathrm{in}$, Equation (7.25) gives a value for the reaction at foundation level as $R=2,112 \mathrm{kips}$, whereas (7.26) indicates $R=2,029$ kips, a difference about $4 \%$. All in all, either Equation (7.25) or Equation (7.26) allows for quick estimation of axial load on the foundation with parameters already known by practitioners. This 4-story LCF building will also be discussed in Chapter (8). 


\subsection{Analytical Examples}

The following example examines the behavior of a 2-story LCF building, using both Cantilever and Vierendeel column approaches. The results are then compared with the design that was made prior experimentally testing the LCF specimen and the design was based on pushover analisis (Lopes et. al., 2014). The building layout is shown in Figure (7.10). A 2-story LCF building with a total height of $220 \mathrm{in}$, story height of $110 \mathrm{in}$ and length of the link equals to $42 \mathrm{in}$ is considered for a total base shear of 216 kips. The total base shear was obtained using the equivalent lateral force procedure as per ANSI/AISC 341 (2010). The location of the building was in Seattle, Washington. Another assumption is that all lateral load contribution is being taken care of by the linked columns with $50 \%$ of the base shear contribution going to each linked column. The building is then considered fixed at the base and is analyzed for a base shear of 108 kips as shown in Figure (7.11).

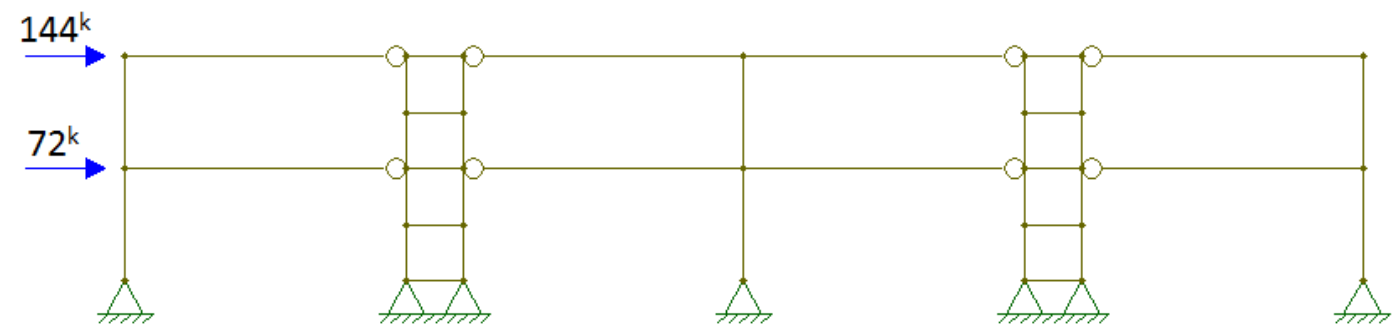

Figure 7.10: 2-story LCF building layout.

In order to limit the total building drift, the LCF system should meet the design intent of $2.5 \%$ inter-story drift limits. The moment of inertia of the 


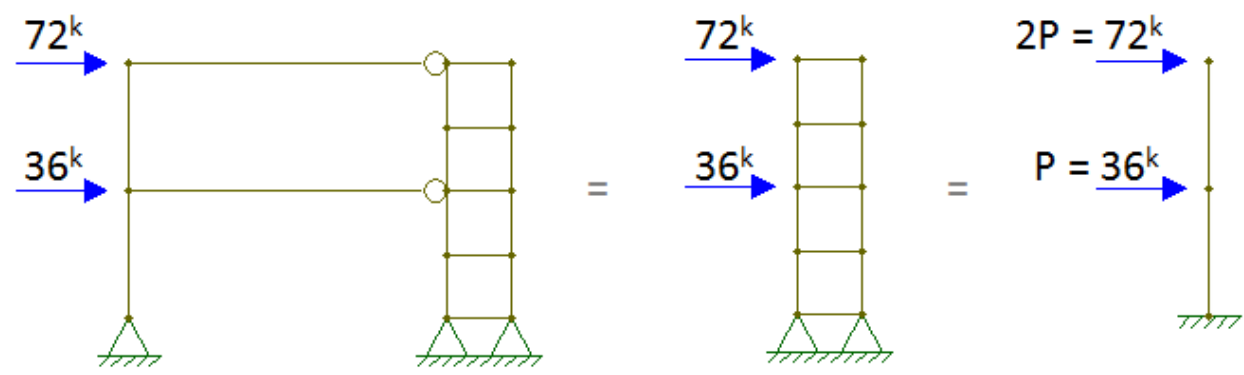

Figure 7.11: Cantilever model for a 2-story LCF building.

linked columns is then calculated from the recurrence relation given by Equation (7.11). The drift limit and the required moment of inertia are $5.5 \mathrm{in}$ and $911 \mathrm{in}^{4}$, respectively.

Using the Design Dimension Tables in the AISC Manual AISC (2011), one might select a W14 x90, the lightest W14 that has at least the required moment of inertia. However, W14x90 is considered to be a non-seismically compact member. Table (7.3) summarizes a set of possible members that could be used in order to proceed with the computer analysis. The other three available sections are also non-seismically compact members.

For seismically compact sections, the width-thickness ratios of the elements of the cross-section cannot exceed $\lambda_{p s}$ as defined in Table I-8-1 per ANSI/AISC 341 (2010). The next lightest section available is W14x132, which was the section used for the experimental tests. At the time, the criterion to choose the section, however, was based upon pushover analyses. 
Table 7.3: Linked Column Member Sizing for a 2-story LCF Building.

\begin{tabular}{|c|c|c|}
\hline W Section & $I_{x}\left(i n^{4}\right)$ & \multirow[b]{2}{*}{ Start with this section } \\
\hline W14x90 & 999 & \\
\hline W14x99 & 1,110 & \multirow{5}{*}{$\stackrel{\text { Final design }}{\longleftarrow}$} \\
\hline W14x109 & 1,240 & \\
\hline $\mathrm{W} 14 \times 120$ & 1,380 & \\
\hline W14x132 & 1,530 & \\
\hline W14 x145 & 1,710 & \\
\hline
\end{tabular}

Knowing the moment of inertia of the linked column member, one obtains the moment of inertia of the link through Equation (7.16). Thus, $I_{L}=208 \mathrm{in}^{4}$ which is the minimum required moment of inertia of the link that should be used in the more refined computer analysis. The links in the experimental design had $I_{L}=248 \mathrm{in}^{4}$

Figure (7.12) shows a 3-story LCF building with a total height of $348 \mathrm{in}$, story height of $116 \mathrm{in}$, length of the link equals to $60 \mathrm{in}$, and a total base shear of 300 kips. The assumptions stated in the previous example are still valid. The building is then considered fixed at the base and is analyzed for a base shear of 150 kips as shown in Figure (7.13).

The drift limit and the required moment of inertia of the linked column 


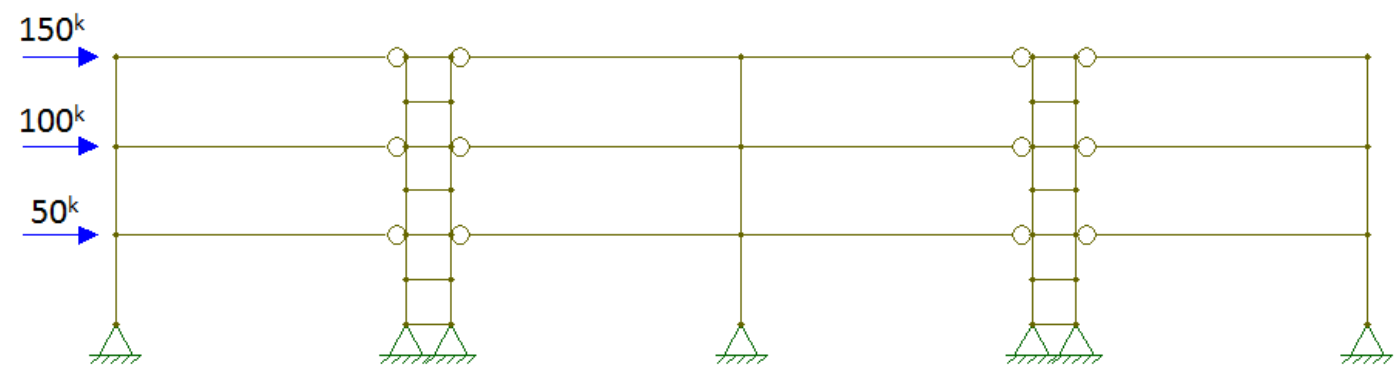

Figure 7.12: 3-story LCF building layout.
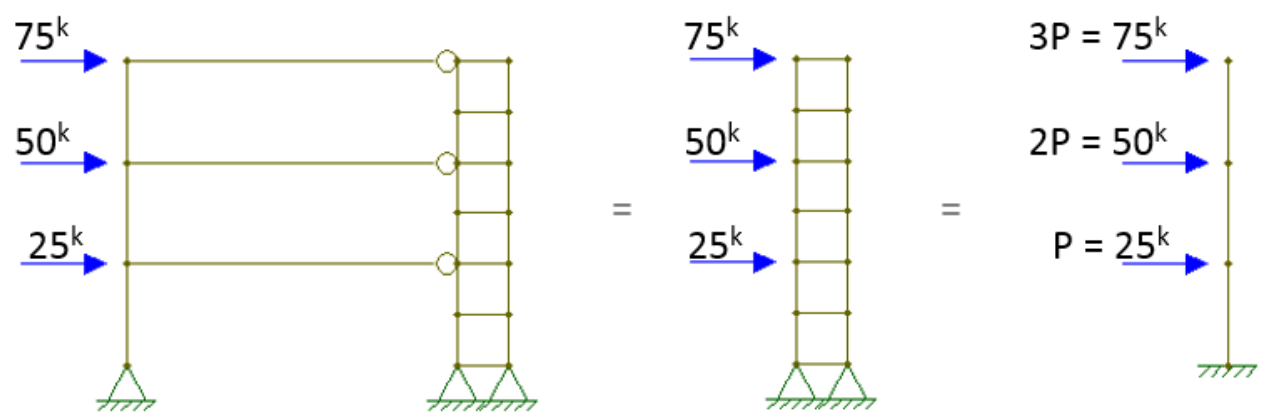

Figure 7.13: Cantilever model for a 3-story LCF building.

given by Equation (7.11) are $8.8 \mathrm{in}$ and 2,996 $\mathrm{in}^{4}$, respectively. Using the Design Dimension Tables in the AISC Manual AISC (2011), one might select a W14x233, the lightest W14 that has at least the required moment of inertia, whereas Malakoutian et. al. (2013) designed a 3-story LCF building with a W14x257 for the linked columns as indicated in Table (7.4). 
Table 7.4: Linked Column Member Sizing for a 3-story LCF Building.

\begin{tabular}{|c|c|c|}
\hline W Section & $I_{x}\left(i n^{4}\right)$ & \\
\hline W14x176 & 2,140 & \\
\hline W14x193 & 2,400 & \\
\hline $\mathrm{W} 14 \times 211$ & 2,660 & \\
\hline $\mathrm{W} 14 \times 233$ & 3,010 & $\stackrel{\text { Start with this section }}{\longleftarrow}$ \\
\hline $\mathrm{W} 14 \times 257$ & 3,400 & $\stackrel{\text { Final design }}{\longleftarrow}$ \\
\hline W14 x283 & 3,840 & \\
\hline
\end{tabular}

Once the moment of inertia of the linked column member is obtained, the moment of inertia of the link is calculated using Equation (7.16). Thus, $I_{L}=930 \mathrm{in}^{4}$ which is the minimum required moment of inertia of the link that should be used in the more refined computer analysis. The links designed by Malakoutian et. al. (2013) had $I_{L}=1,330 \mathrm{in}^{4}$.

As a third and final example, Figure (7.14) shows a 6-story LCF building with a total height of $756 \mathrm{in}$, story height of $126 \mathrm{in}$, length of the link equals to $80 \mathrm{in}$, and a total base shear of 336 kips. The assumptions stated in the previous example are still valid. The building is then considered fixed at the base and is analyzed for a base shear of 168 kips as shown in Figure (7.15).

The drift limit and the required moment of inertia of the linked column given by Equation (7.11) are $18.9 \mathrm{in}$ and $13,860 \mathrm{in}{ }^{4}$, respectively. Using the 


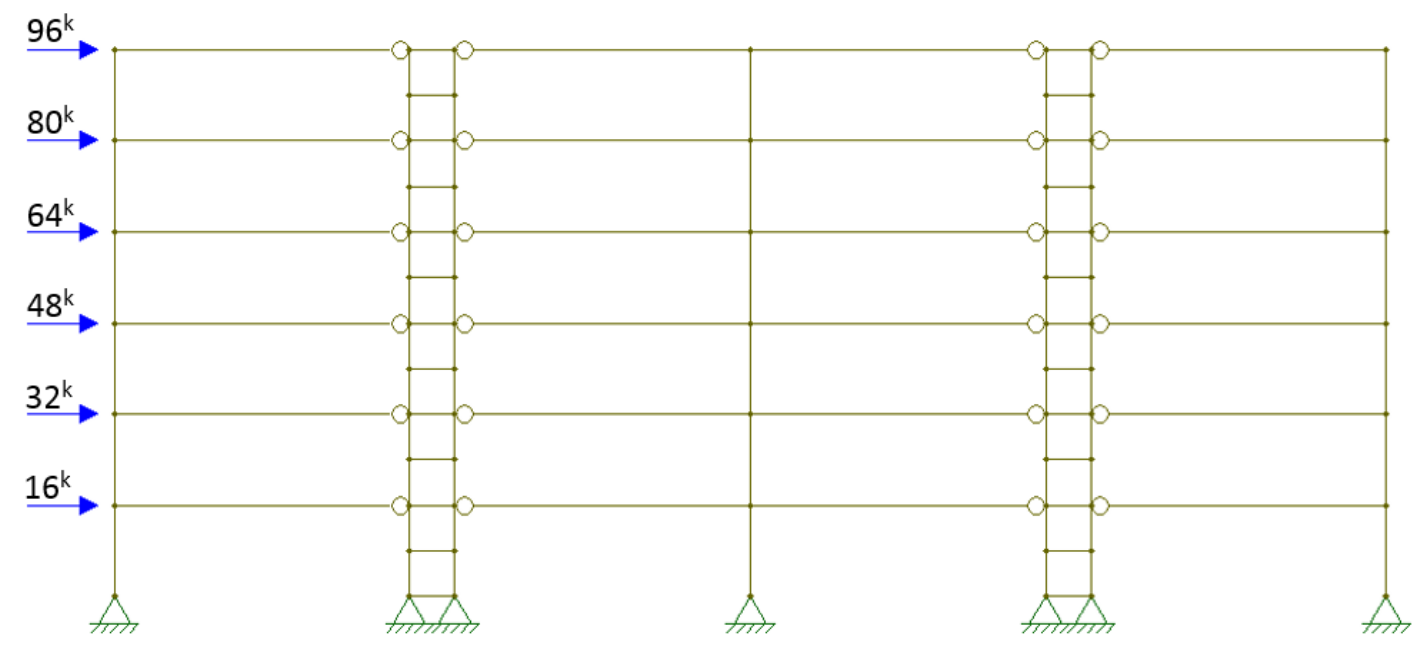

Figure 7.14: 6-story LCF building layout.

Design Dimension Tables in the AISC Manual AISC (2011), one might select a W14x730, the lightest W14 that has at least the required moment of inertia which is the same cross section chosen by Malakoutian et. al. (2013) as indicated in Table (7.5).

Table 7.5: Linked Column Member Sizing for a 6-story LCF Building.

\begin{tabular}{|c|c|}
\hline W Section & $I_{x}\left(\mathrm{in}^{4}\right)$ \\
\hline $\mathrm{W} 14 \times 550$ & 9,430 \\
\hline $\mathrm{W} 14 \times 605$ & 10,800 \\
\hline $\mathrm{W} 14 \times 665$ & 12,400 \\
\hline $\mathrm{W} 14 \times 730$ & 14,300 \\
\cline { 1 - 2 }
\end{tabular}

Similarly to the previous examples, once the moment of inertia of the linked column member is obtained, the moment of inertia of the link is calculated 182 


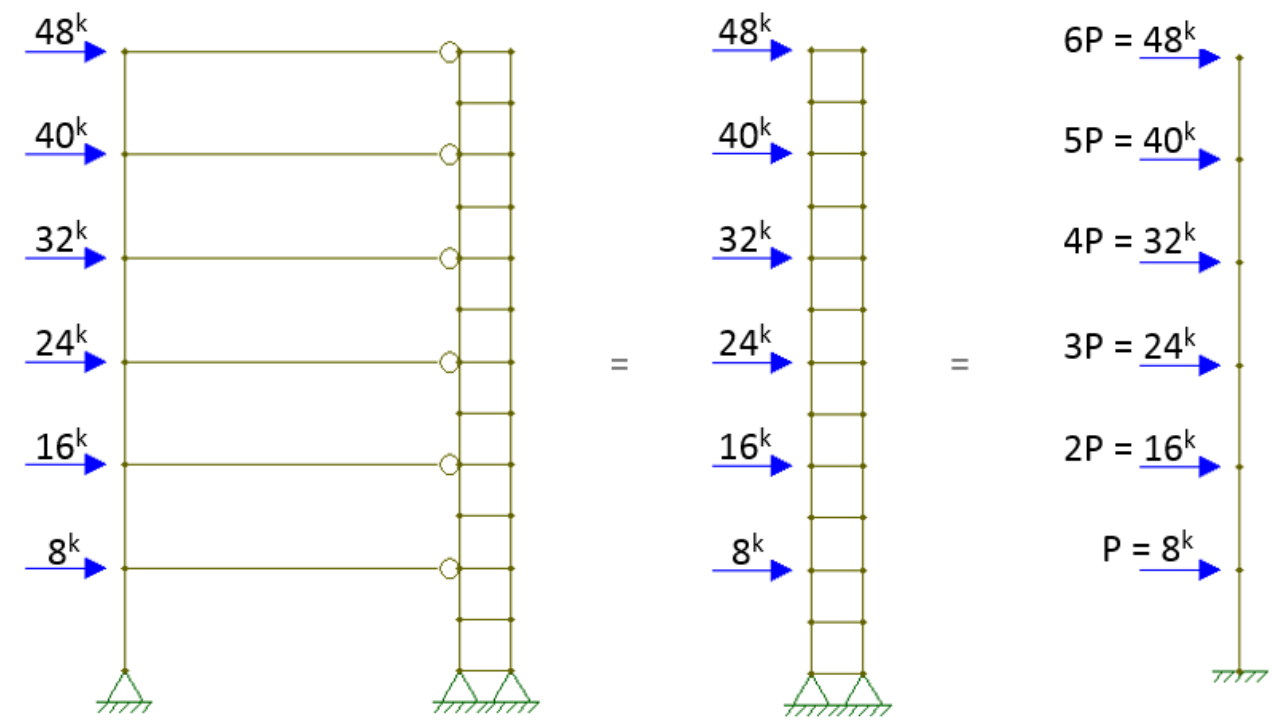

Figure 7.15: Cantilever model for a 6-story LCF building.

using Equation (7.16). Thus, $I_{L}=5,280 \mathrm{in}^{4}$ which is the minimum required moment of inertia of the link that should be used in the more refined computer analysis. The links designed by Malakoutian et. al. (2013) had $I_{L}=5,680 \mathrm{in}^{4}$. Table (7.6) summarizes the member sizing using both Cantilever and Vierendeel approaches and compares to the LCF buildings that were already designed.

Table 7.6: Summary of the member sizing.

\begin{tabular}{|c|c|c|c|c|}
\hline \multirow{2}{*}{$\begin{array}{c}\text { LCF number } \\
\text { of stories }\end{array}$} & \multicolumn{2}{|c|}{$I_{L C}\left(i n^{4}\right)$} & \multicolumn{2}{c|}{$I_{L}\left(i n^{4}\right)$} \\
\hline 2 & 911 & 1,530 & 208 & 248 \\
\hline 3 & 2,996 & 3,400 & 930 & 1,330 \\
\hline 6 & 13,860 & 14,300 & 5,280 & 5,680 \\
\hline
\end{tabular}

LCF systems were investigated analytically via structural mechanics in order 
to select preliminary member sizes to be used for computer model generation, which would subsequently be used for more detailed design. An apparently simple formulation was presented for the development of closed-form expressions of the lateral stiffness of the linked column steel frame system and also to size gravity beam members. In regards to the lateral stiffness, two approaches were presented based on classical methods of structural analysis.

The analytical examples demonstrated that the prediction of the member sizes is reasonable and illustrated the applicability and accuracy of the proposed formulations. By the same token, expressions developed for a quick estimation of the axial reaction on the foundation element were also presented. Although the apparent advantage of the approaches presented lies in the use of simple equations, member sizes should be adjusted accordingly to satisfy strength criteria. 


\section{Chapter 8}

\section{Design Practice for LCF}

LCFs combine the advantages of high ductility and lateral stiffness by limiting the inelastic behavior to ductile links and by keeping the rest of the frame essentially elastic. LCFs are designed so that yielding during a seismic event is restricted primarily to the ductile links. The overall goal of this chapter is to develop guidelines for preliminary LCF design that will result in configurations where linked columns and links are likely to satisfy capacity design requirements. Also, a step-by-step design example is presented in order to take advantage of an existing structural design practice.

\subsection{System Geometry}

The building architectural design plays a major role in determining the building's seismic performance. Ratios of span to height can often be relied upon to provide a guide and a starting point from which further refinement can be made. A reasonable solution is often required as a computer input. However, the validity of the computer output should always be verified. 


\subsubsection{Floor Height}

From experience, it has been observed that the practical limit of floor height for conventional steel structures is about 13 inches.

\subsubsection{Span Length}

For most steel frames, the span length varies between 20 and 30 feet.

\subsubsection{Number of Linked Columns}

The number of linked columns, $N_{L C}$ is defined by Equation (8.1)

$$
N_{L C}=\frac{N_{\text {Bays }}}{2}
$$

where $N_{\text {Bays }}$ is the number of bays and $N_{L C}$ should be equal to as least one.

\subsubsection{Link Length}

Selection of link length is often restricted by architectural restraints. In the absence of those restraints, preliminary link length is calculated using Equation

$$
L_{L}=0.2 L_{G B}
$$

where $L_{G B}$ is the gravity beam length. 


\subsubsection{Steel Floor Systems}

In steel floor systems, reinforced concrete slabs with shear connection on steel beams are often used with thickness of slabs in the range of $\mathrm{L} / 30$ to $\mathrm{L} / 15$ of the span.

\subsection{Loading Conditions}

One of the most difficult and yet important steps in the overall process of design is the definition of the loads. The loads that were considered in the LCF design can be grouped in three categories: dead load, live load and earthquake load. All loads could be combined before the analysis is performed and, for LRFD, the required strength is determined from Equation (8.3).

$$
P_{u}=1.2 D+0.5 L \pm 1.0 E
$$

where $P_{u}$ is the required strength, $D$ is dead load, $L$ is live load, and $E$ is earthquake load. The first phase of structural design consists of estimating the loads acting on the structure. This is done using the load values and combinations presented in ASCE/SEI 7 (2010).

In regards to earthquake loading, usually, the distribution of lateral forces is associated with the first mode of vibration of a structure modeled as a cantilever. Thus, the equivalent lateral force procedure (ASCE/SEI 7, 2010) is an option. This procedure consists of determining the base shear in function of the seismic 
response coefficient, $C_{S}$, and the effective seismic weight, $W$, including the total dead load of the building, according to Equation (8.4). The idea behind the equivalent lateral force procedure is to position static loads on a building with values and direction that approximate the effects of dynamic loading caused by earthquakes. For the LCF design, the proportion of structural members is performed once the lateral force distribution is completed.

$$
V=C_{S} W
$$

\subsection{Proportion Structural Members}

The LRFD method of proportioning structural members and their connections is used. The method consists of using load and resistance factors such that no applicable limit state is reached when the structure is subjected to appropriate load combinations.

\subsubsection{Linked Columns}

The moment of inertia of a single linked column is obtained using Equation

$$
I_{L C}=\frac{x^{4.6} P h^{3}}{8 E \Delta}
$$

where $I_{L C}$ is the moment of inertia for a single linked column, $x$ is the 
number of stories, $P$ is the lateral seismic force, $\mathrm{h}$ is the story height of the LCF building, $E$ is the modulus of elasticity, and $\Delta$ is the lateral deflection caused by a seismic force. The derivation of Equation (8.5) is based on the LCF Cantilever Method.

\subsubsection{Links}

The moment of inertia of a shear link is obtained using Equation (8.6)

$$
I_{L}=\frac{0.6 I_{L C} H}{h}
$$

where $I_{L}$ is the moment of inertia for a link and $H$ is the length of the link. The derivation of Equation (8.6) is based on the LCF Vierendeel Method. Also, links should be designed as shear links for eccentrically braced frames.

\subsubsection{Gravity Beams and Gravity Columns}

The gravity beams are primarily loaded in bending about a primary axis of the member. However, depending on the lateral loads of the system there are beams with axial loads as well. The basic design checks for beams includes checking bending, shear and deflection. The typical design procedure for beams involves selecting a member that has adequate strength in bending and shear, and adequate stiffness for serviceability. The loading conditions and beam configuration will dictate which of the preceding design parameters controls the size of the 
beam. A practical equation to determine the required moment of inertia of the gravity beam for $L / 360$ is given by:

$$
I_{G B}=\frac{4 w L^{3}}{5}
$$

where $w$ is the live load and $L$ is the length of the beam.

As mentioned before, the LCF structural members are subjected to combined axial and bending loads, and such members are called beam-columns. Because of the many variables involved, no simple design procedure is likely to account for such varied behavior. The design of beam-columns is essentially a trial-and-error process, as such AISC (2011) formulas for beam-columns are

of the interaction type. Interaction equations come closer to describing the true behavior since they account for stability situations commonly encountered. For practical purposes, once the linked column size is determined consider the gravity column having the same cross section.

\subsection{Strong Column and Weak Beam Design}

When a building sways during an earthquake, the distribution of damage over height depends on the distribution of lateral drift. If the building has weak columns, drift tends to concentrate in one or a few stories. However, if columns are stronger than the beams, drift will be more uniformly distribute. Figure 
(8.1) shows two different mechanisms: (a) story mechanism and (b) beam mechanism. The former mechanism should be avoided at all costs during the LCF design while the latter mechanism is preferable in order to spread inelastic response over several stories. ANSI/AISC 341 (2010) adopts a strong-column and weak beam design approach that requires that the summation of column flexural strengths at each joint exceed the summation of beam flexural strengths. Practitioners may want to increase columns sizes, beyond the code requirements in order to obtain better performance in severe earthquake events and to avoid the need of web stiffeners and doubler plates, but will increase, however, the total weight of the steel used on the project.

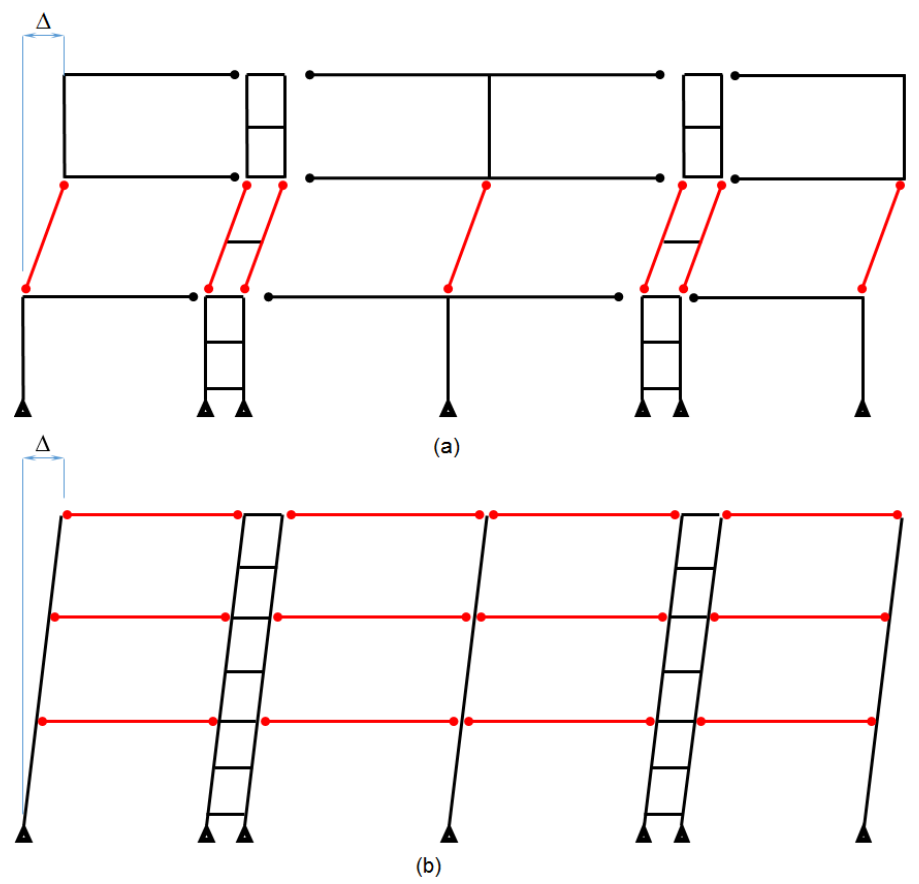

Figure 8.1: (a) story mechanism and $(b)$ beam mechanism. 


\subsection{Serviceability and Drift}

For beams, deflections must be limited such that the occupants of the structure recognize that the structure is safe. The deflection equations for common loading conditions are found in IBC (2012) and are summarized in Table (8.1).

Table 8.1: Deflection limit for beams.

\begin{tabular}{rcc}
\hline Member description & Live Load & Dead + Live Load \\
\hline Roof: supporting plaster ceiling & L/360 & L/240 \\
Roof: supporting non-plaster ceiling & L/240 & L/180 \\
Roof: not supporting plaster & L/180 & L/120 \\
Floor members & L/360 & L/240 \\
\hline
\end{tabular}

Sizing of columns in LCF systems typically is controlled by consideration of drift. The stiffness of the LCF system must be sufficient to control the drift of the building at each story within the limits specified by the building code. Drift limits in ASCE/SEI 7 (2010) are a function of both type of structure and risk category. The drift of the structure is to be calculated using the factored seismic load, amplified by $C_{d}$, when comparing it with the values in ASCE/SEI 7 (2010). Therefore, practitioners should adjust member sizes accordingly checking interstory drifts. Should drift criteria not be met, increase the stiffness of the shear links and linked columns first before increasing the stiffness of the gravity beams. 


\subsection{Pushover Analysis}

A preliminary pushover analysis is often necessary to enable comparisons with inelastic time-history analysis. However, there are still some reservations about the inelastic time-history analysis, which are mainly related to its complexity and suitability for practical design applications due to its sensitivity to the characteristics of the input motions. On the other hand, pushover analysis involves only a predefined lateral load pattern which is distributed along the building height, a target displacement and a chosen level of performance. The expectation is that the results will provide a sequence of yielding and failure on the structural members, as shown in Figure (8.2), as well as the progress of the overall capacity curve of the structure in order to predict the inelastic forcedeformation behavior of the structure. Pushover analysis is selected because of its applicability to performance-based seismic design approaches. Moreover, pushover analysis is one of methods described and recommended in FEMA 440 (2005).

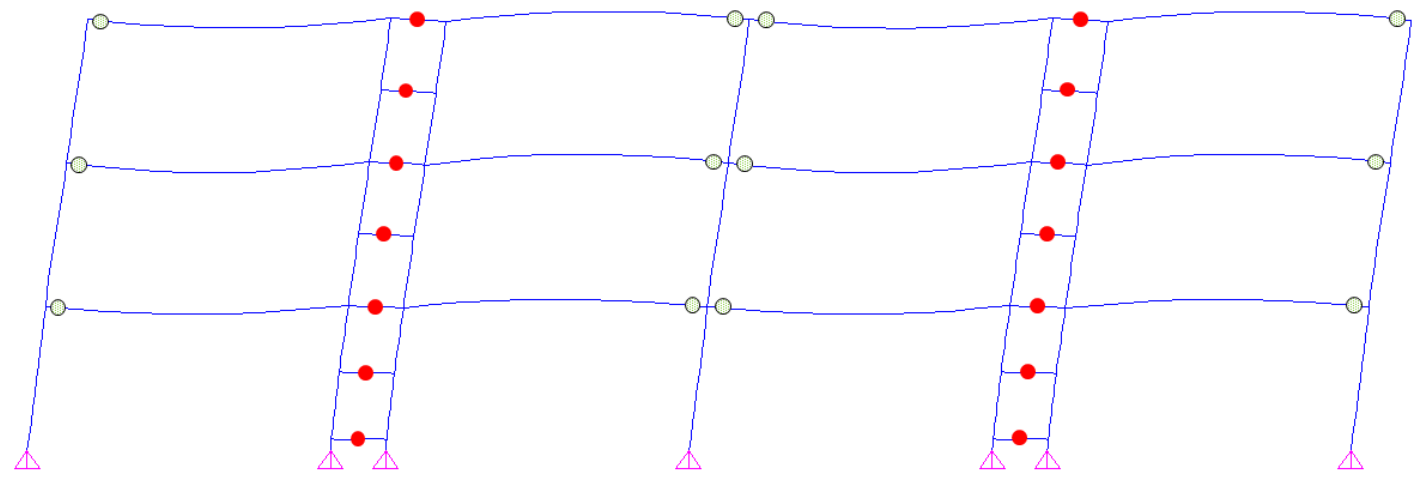

Figure 8.2: LCF building sequence of yielding: links and then gravity beams. 


\subsection{Performance Levels}

New structural systems are needed to target specific performance levels especially for lower than maximum expected events. The LCF system is a new lateral load resisting system with inherent specific target performance levels and the ability for rapid return to occupancy following an earthquake while maintaining the appeal of non-braced frame system. There are three design performance levels, as shown in Figure (8.3), in regards to suitability of the LCF building for occupancy and function:

1. Immediate occupancy where the system remains linearly elastic. No damage has occurred and repair is not required. $50 \%$ of exceedence in 50 years.

2. Rapid return to occupancy where only the shear links would yield. Moderate damage has occurred and is limited to parts of the structure. Repair is required in terms of removing and replacing any permanently deformed shear links to restore the self-centering mechanism. In other words, the building would be repairable at low costs. $10 \%$ of exceedence in 50 years.

3. Collapse prevention where the gravity beam components also become damaged. Moderate and severe damage had occurred. Building is unsafe for occupancy and would be repairable, although not economically. $2 \%$ of exceedence in 50 years. 


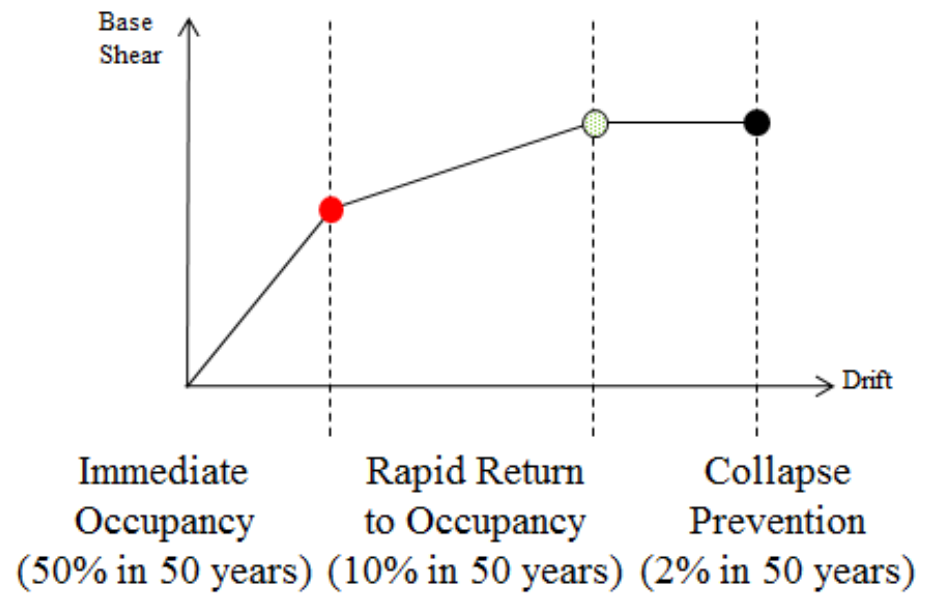

Figure 8.3: Limit states of a LCF building.

The effectiveness of the rapid return to occupancy performance level depends on the relative transitions from elastic to plastic response of the $\mathrm{LC}$ and of the MF. Relative displacements, given by Equation (8.8), should be computed in order to guarantee the potential for rapid return to occupancy performance level.

$$
\frac{\Delta_{L C}}{\Delta_{M F}}=\frac{V_{L C} K_{M F}}{V_{M F} K_{L C}}<1
$$

Ratios less than unity describe structural systems in which LC reaches plasticity at lower displacements than MF, thereby helping the system to achieve the rapid return to occupancy performance level. Should relative displacements not be met, increase the stiffness of the shear links and linked columns first before increasing the stiffness of the gravity beams.

At this point, several pushover analyses were already carried out and the 
output is a pushover curve (drift or displacement vs base shear). From the pushover curve for the LCF system first yield point of the shear link is obtained. This value is then compared to the base shear design computed using the equivalent lateral force procedure. Ratio approximate to unity indicates lower foundation forces. A practical value of 1.2 is still acceptable.

$$
\frac{V_{L C}}{V_{\text {design }}} \approx 1.2
$$

Hence, LCF systems are designed to have a base shear closer to the design at first link yield. This also ensures a reduction of the system overstrength factor, $\Omega_{0}$, since the first beam yield would be reduced as well. One of the advantages of the LCF system is that the practitioners have control of this parameter. Quantification of the actual overstrength can be employed to reduce the forces used in the design, therefore, leading to more economical structures.

\subsection{Design Example}

The advantage of using a LCF system relies on the combination of traditional structural elements, such as beams and columns, and the use of an existing structural design practice. The construction of the LCF system is not difficult and, despite of being novel, the system is still implementable. The design of a typical LCF system can be performed by following the flowchart given in Figure (8.4) and is illustrated through an example of a 4-story steel frame building with 
LCFs for its lateral force resisting system. 


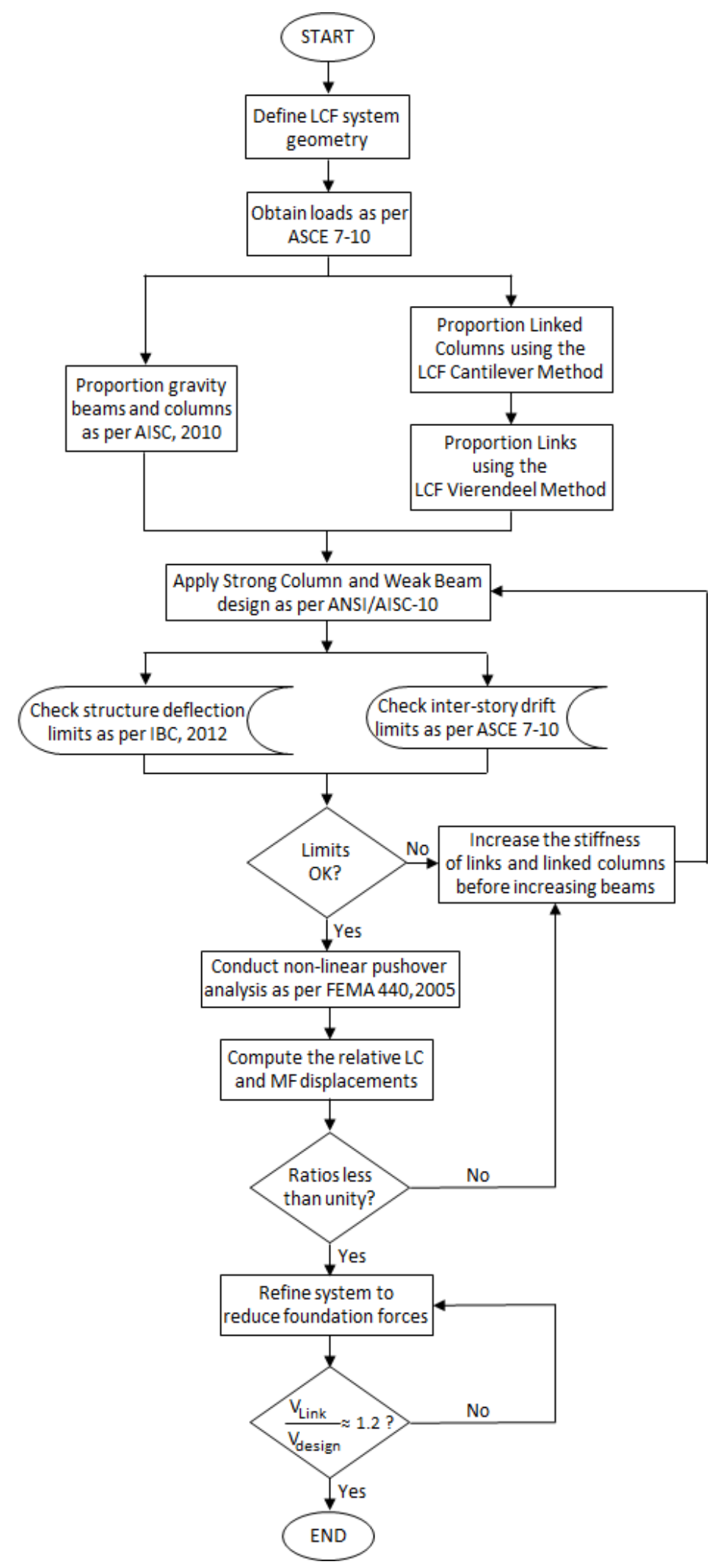

Figure 8.4: Linked Column Frame Flowchart. 


\section{System Geometry}

The LCF sample building site is located in Seattle, Washington. The floor plan of this 4-story building consists of 4-bays in the North-South direction and 4-bays in the West-East direction.

The building is square in plan with a total plan area of $14,400 \mathrm{ft}^{2}$ and the plan consists of a $30 \mathrm{ft}$ by $30 \mathrm{ft}$ typical bays. The building plan and LCF elevation are shown in Figures (8.5) and (8.6), respectively. The LCF studied has a story height of $13 \mathrm{ft}$ and spacing between linked-columns of $H=5 \mathrm{ft}$.

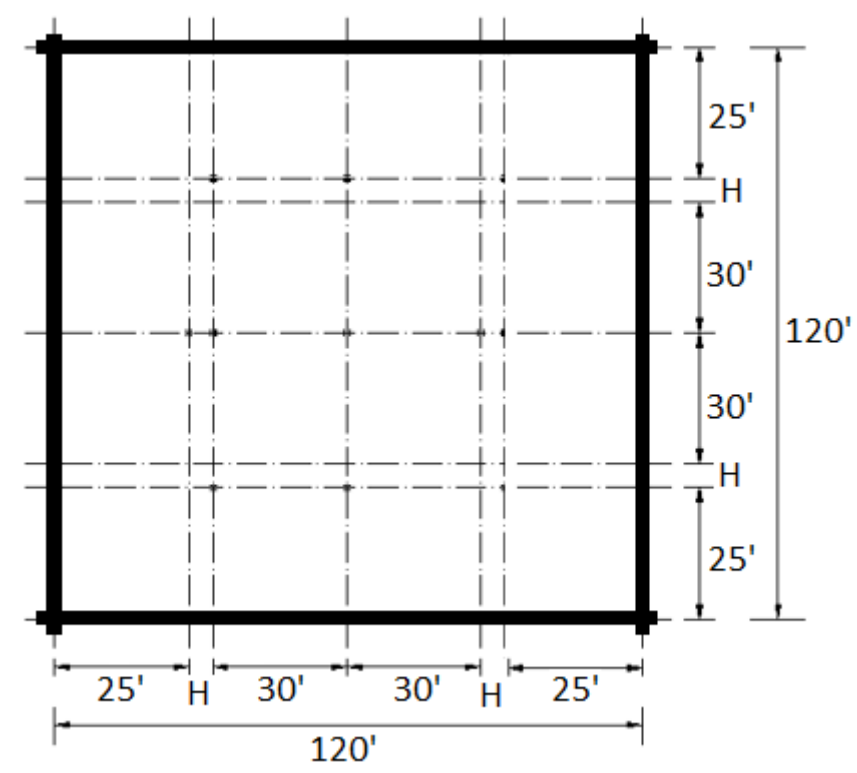

Figure 8.5: Plan view of LCF sample building. 


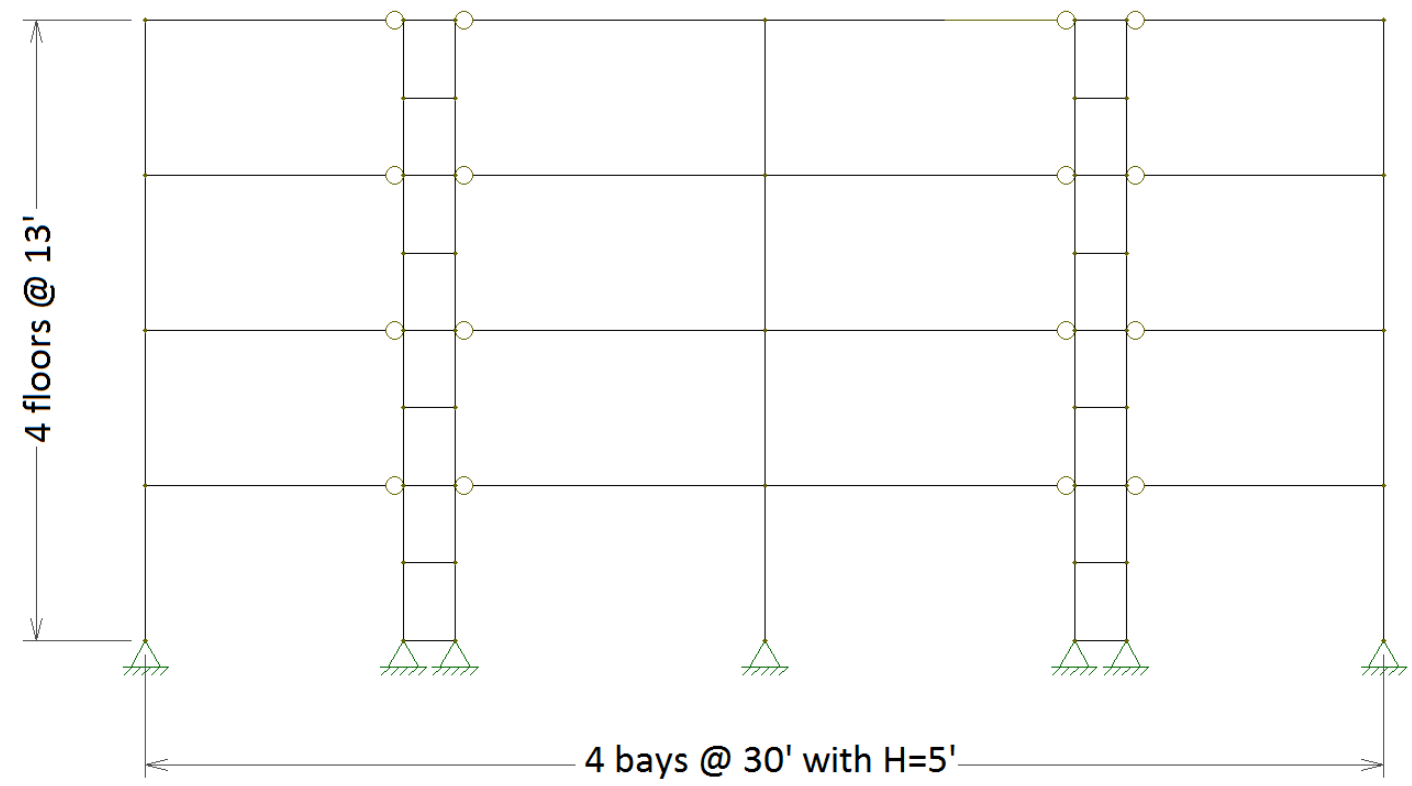

Figure 8.6: Elevation view of LCF sample building.

\section{Loading Conditions}

The loading used for the analysis of the LCF system is based on the details given in FEMA 355-C (2000), which results in the following floor load distribution:

Floor dead load for weight calculations : 96psf

Floor dead load for mass calculations : : 86psf

Roof dead load : $\quad$ : 3 psf

Reduced live load per floor and for roof : 20psf

Roof seismic mass $\quad$ : $70.90 \mathrm{kips}^{-\mathrm{sec}^{2} / \mathrm{ft}}$

Floor seismic mass $\quad: 65.53 \mathrm{kips}^{-\mathrm{sec}^{2} / \mathrm{ft}}$

Corresponding seismic hazard parameters are given in Table (8.2). 
Table 8.2: Seismic design parameters for the LCF sample building.

\begin{tabular}{|c|c|}
\hline Parameter & Value \\
\hline $\mathrm{R}$ & 8 \\
\hline$\Omega$ & 2 \\
\hline $\mathrm{C}_{\mathrm{d}}$ & 5.5 \\
\hline Occupancy Category & 1.0 \\
\hline Importance Factor & 1.0 \\
\hline Soil Profile Type & B \\
\hline $\mathrm{S}_{\mathrm{MS}}$ & $1.3 \mathrm{~g}$ \\
\hline $\mathrm{S}_{\mathrm{M} 1}$ & $0.443 \mathrm{~g}$ \\
\hline $\mathrm{S}_{\mathrm{DS}}$ & $0.867 \mathrm{~g}$ \\
\hline $\mathrm{S}_{\mathrm{D} 1}$ & $0.295 \mathrm{~g}$ \\
\hline Building Height & $39 \mathrm{ft}$ \\
\hline $\mathrm{C}_{\mathrm{t}}$ & 0.02 \\
\hline $\mathrm{x}$ & 0.75 \\
\hline Approximate Period & $0.39 \mathrm{sec}$ \\
\hline $\mathrm{C}_{\mathrm{s}}$ & $0.095 \mathrm{~g}$ \\
\hline Building Area & $14,400 \mathrm{ft}^{2}$ \\
\hline Effective Seismic Weight & 5,342 kips \\
\hline Base Shear & 260 kips \\
\hline Maximum Drift Ratio & $2.5 \%$ \\
\hline
\end{tabular}

Since most structures are multiple degrees-of-freedom systems with several modes of vibration, the distribution of the seismic lateral force is a combination of the contributions from all significant modes of vibration of the structure. 
The force distribution to each level is a function of the seismic weight, at that level, the height of the structure and the predominant mode of vibration. The corresponding lateral force distribution is given in Table (8.3). The previous calculations, based on the equivalent lateral force procedure, were necessary in order to obtain a value for the base shear.

Table 8.3: Lateral Force Distribution Calculations for the LCF sample building.

\begin{tabular}{cccccccc}
\hline Level & $w_{\mathrm{x}}($ kips $)$ & $\sum w($ kips $)$ & $h_{\mathrm{x}}($ kips $)$ & $h($ kips $)$ & $w_{\mathrm{x}} \cdot h_{\mathrm{x}}$ & $\%$ & $F_{\mathrm{x}}($ kips $)$ \\
\hline Roof & 1196 & 1196 & 52 & 13 & 62192 & 37 & 96 \\
Floor 3 & 1382 & 2578 & 39 & 13 & 53898 & 31 & 82 \\
Floor 2 & 1382 & 3960 & 26 & 13 & 35932 & 21 & 56 \\
Floor 1 & 1382 & 5342 & 13 & 13 & 17966 & 11 & 26 \\
$\sum$ & 5342 & - & - & - & 169,988 & 100 & 260 \\
\hline
\end{tabular}

\section{Proportion Structural Members}

Proportioning linked columns and shear links is performed after the lateral force distribution is obtained. Figure (8.7) shows a 4-story building layout with its corresponding lateral forces. Note that at foundation level all supports are considered to be pinned and the total base shear is 260 kips.

The next step is to isolate each LCF sub-system and divide the base shear equally among all LCF sub-systems. Figure (8.8) shows the cantilever model for a 4-story LCF building with a total total base shear of 130 kips. Note that at foundation level, on the cantilever model, supports were changed from pin to fix 
support. The value of $P$ acting on the first floor of the building is $P=13$ kips.

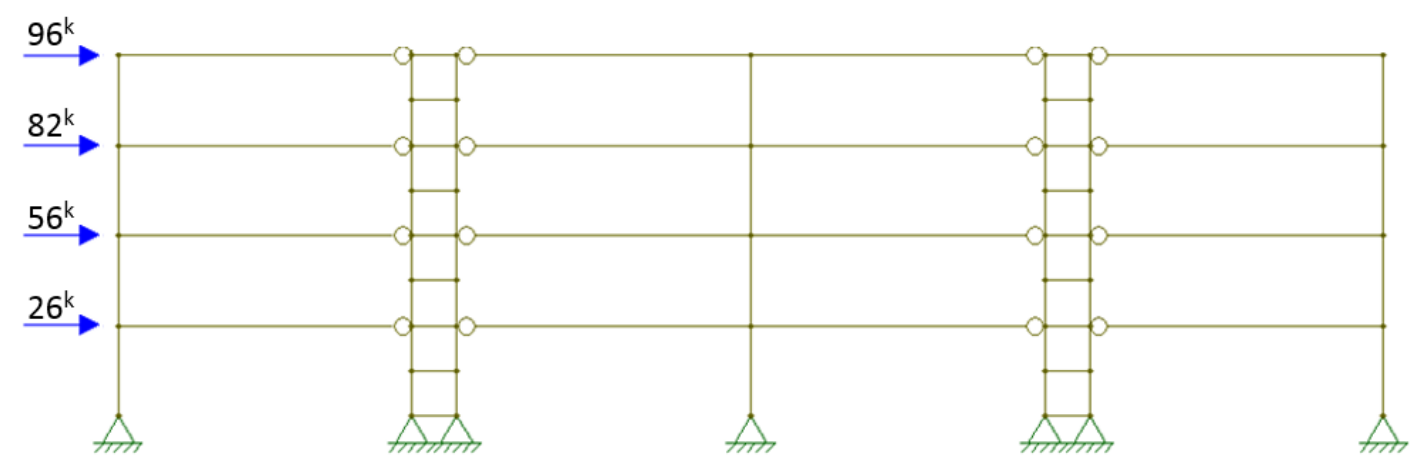

Figure 8.7: 4-story LCF building layout.
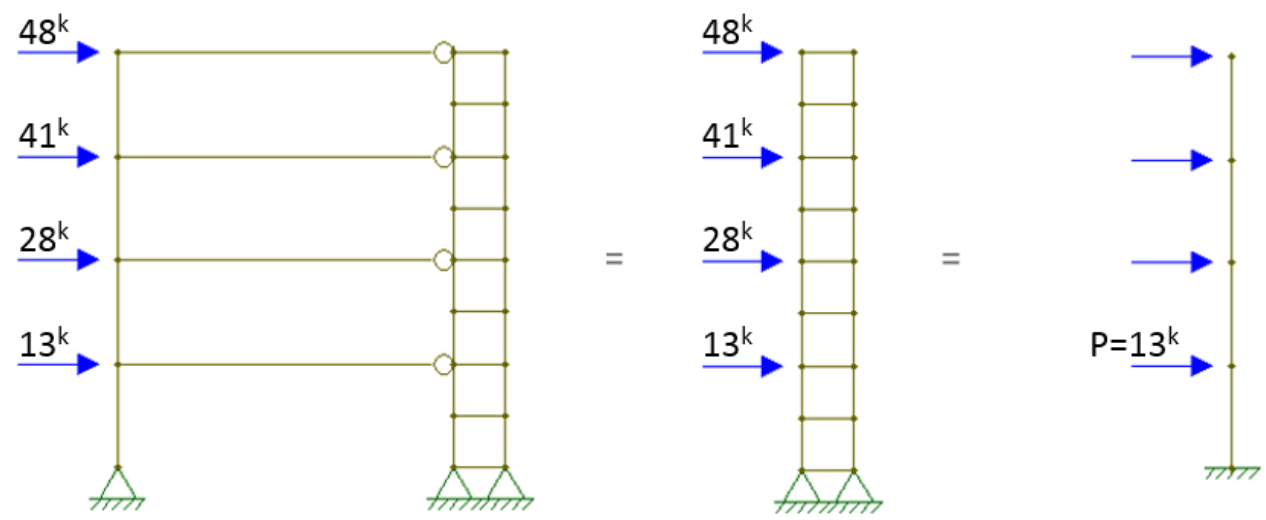

Figure 8.8: Cantilever model for a 4-story LCF building.

For the sake of understanding, equations developed in Chapter (7) are used to determine the preliminary sections for columns, links and beams, as follows. First, determine the target displacement at the top of the building to be used in Equation (8.11) in order to calculate the moment of inertia of a single linked column.

$$
\Delta=\frac{2.5}{100} L_{\text {total }}=\frac{2.5}{100}(624)=15.6 \text { in }
$$




$$
I_{L C}=\frac{x^{4.6} P h^{3}}{8 E \Delta}=\frac{4^{4.6}(13)(156)^{3}}{8(29,000)(15.6)}=8,020 i n^{4}
$$

The next step is to proportion the shear links in function of the moment of inertia of a single linked column. Thus, the moment of inertia for a shear link is given by:

$$
I_{L}=\frac{0.6 I_{L C} H}{h}=\frac{0.6(8,020)(60)}{156}=1,850 \mathrm{in}^{4}
$$

For the gravity beam, use the following equation based on serviceability:

$$
I_{G}=\frac{4 W L^{3}}{5}=\frac{4(0.03)(27.5)^{3}}{5}=500 \mathrm{in}^{4}
$$

With these moments of inertia, previously obtained, choose a proper section from the Design Dimension Tables in the AISC Manual AISC (2011).

Table (8.4) summarizes a set of possible seismically compact wide flanges, denoted hereafter as first model, that could be used in order to proceed with the computer analysis. Note that the moments of inertia are slightly greater than the ones calculated. A total of three numerical models were analyzed in order to optimize the LCF design.

Table 8.4: First model sizing for a 4-story LCF Building.

\begin{tabular}{cc}
\hline W Section & $I_{x}\left(i n^{4}\right)$ \\
\hline W14x500 & 8,210 \\
W14x159 & 1,900 \\
W16x40 & 518 \\
\hline
\end{tabular}




\section{Strong Column and Weak Beam Design}

The strong column and weak beam design requires that, at a node, the summation of the column's plastic moment capacities exceed the summation of the beam's plastic moment capacities, based on simple moment equilibrium at the node.

Consider, for example, the sizing members for the first model. Hence, the acceptance criteria is given by Equation (8.14) and calculations are shown in Figure (8.9).

$$
\frac{\sum M p_{c}}{\sum M p_{b}}>1.0=\frac{7,115}{4,495}=1.6
$$




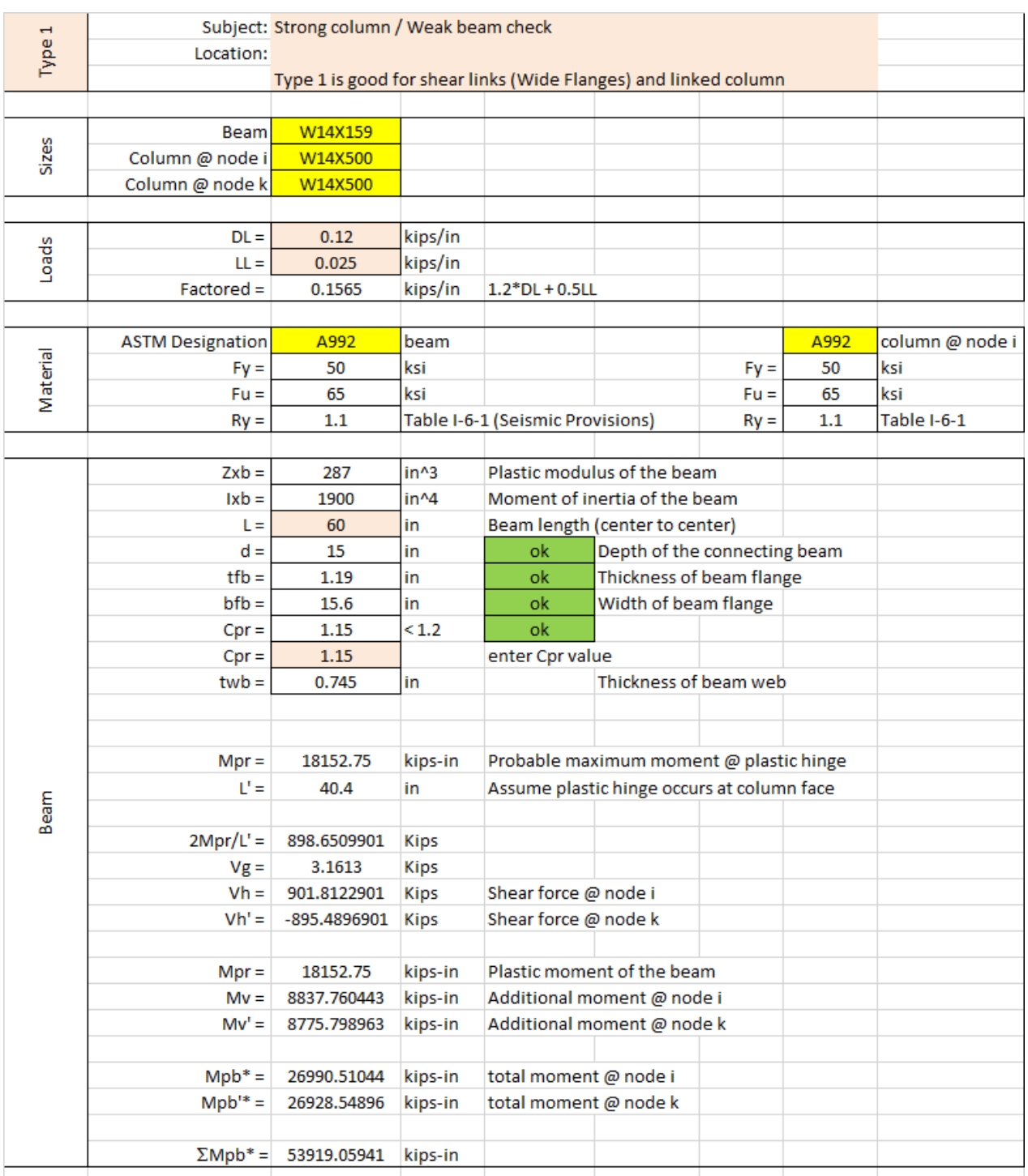

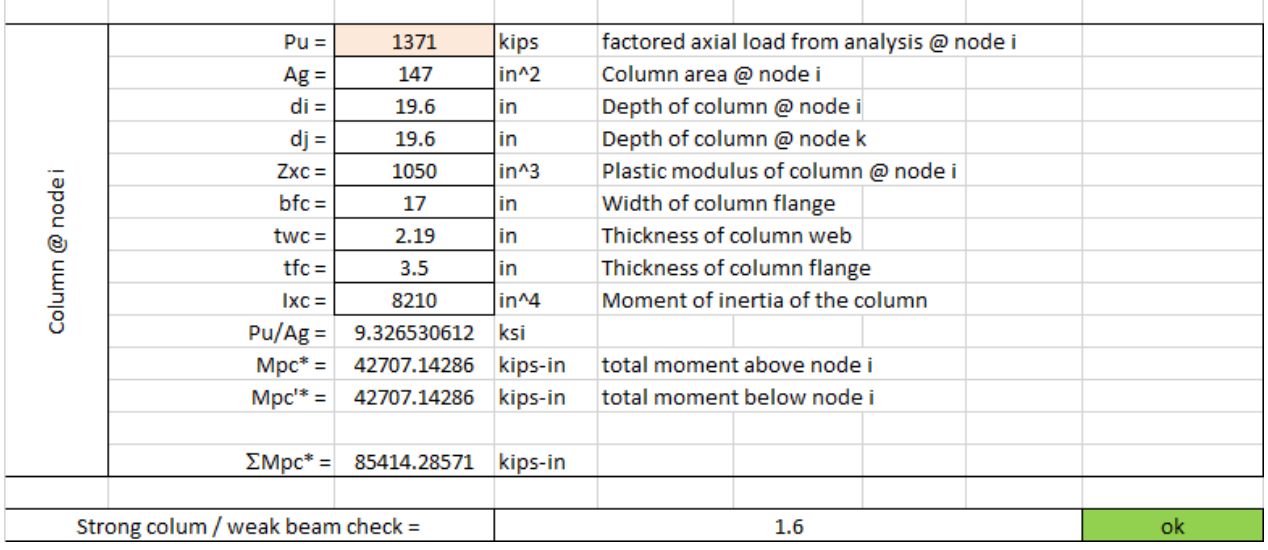

Figure 8.9: First model strong column and weak beam design calculations. 


\section{Serviceability and Drift}

In addition to being safe, a structure must be serviceable. The gravity beam was already chosen considering serviceability requirements, which means that deflections were limited to avoid vibration problems and also to avoid very flexible beams.

A two-dimensional analysis of the structure was performed under the loading conditions, previously described, and to account for accidental torsion, the mass of each level was assumed to be displaced from the center of mass by a distance equal to $5 \%$ of the building dimension perpendicular to the direction of force.

Rigid diaphragms were assigned at each level as well as rigid-end offsets were defined at the ends of each member. Also, $\mathrm{P}-\Delta$ effects were considered in the lateral analysis. The lateral displacements of the design example building, computed elastically under the distributed lateral forces of Table (8.3), are shown in Table (8.5), where $h$ is the story height of the building, $\delta_{x e}$ is the

elastic displacement, $\Delta_{x e}$ is the elastic drift, $\delta_{x}$ is the design drift, and $\Delta_{a}$ is the drift limit. 
Table 8.5: First model drift analysis for the LCF sample building.

\begin{tabular}{rcccccc}
\hline & $\mathrm{h}$ & $\delta_{x e}$ & $\Delta_{x e}$ & $\delta_{x}$ & $\Delta_{a}$ & $\%$ \\
\hline Roof & 156 & 2.11 & 0.47 & 2.59 & 3.9 & 50 \\
Floor 3 & 156 & 1.64 & 0.54 & 2.97 & 3.9 & 31 \\
Floor 2 & 156 & 1.10 & 0.56 & 3.08 & 3.9 & 27 \\
Floor 1 & 156 & 0.54 & 0.54 & 2.97 & 3.9 & 31 \\
\hline
\end{tabular}

For the first model, shear links were placed only at story levels and all of them were wide flanges. Should drift limits are not satisfied, increase the stiffness of links and linked columns before increasing beams. Another option is to increase the number of links, for example, place extra shear links at mid-height story levels.

Table (8.5) indicates that the first model satisfied the drift limit, however, the difference between $\Delta_{a}$ and $\delta_{x}$, at roof level, is about $50 \%$. This difference shows that the structure should be optimized in order to obtain a more economical LCF structure. Thus, two additional models are presented regarding structural optimization. The main difference between them is that one model has variable wide flange shear links along the height of the LCF building, denoted hereafter as second model, while the other one has variable built-up sections for shear links, denoted hereafter as third model. Figure (8.10) shows the labeling that were used in the analyses. 


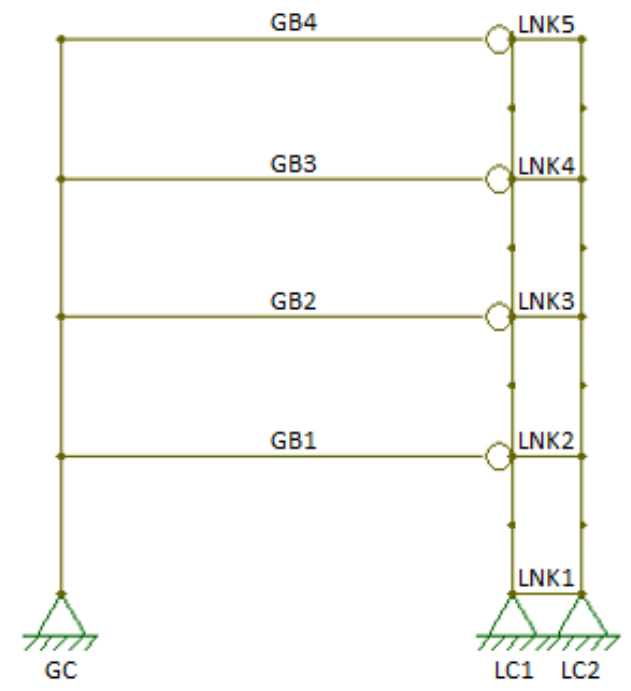

Figure 8.10: LCF substructure labeling.

Note that labeling presented in Figure (8.10) represents only one LCF substructure. The remaining of the building was still modeled and cross section members for the moment frame, that connects the two LCF substructures, have the same section properties. Table (8.6) presents the member sizing that were used in all analyses while Table (8.7) presents the shear link section properties used on the third model. 
Table 8.6: Member sizing for the LCF sample building.

\begin{tabular}{cccc}
\hline Member & $1^{\text {st }}$ model & $2^{\text {nd }}$ model & $3^{\text {rd }}$ model \\
\hline GC & W14x500 & W14x605 & W14x605 \\
LC1 & W14x500 & W14x605 & W14x605 \\
LC2 & W14x500 & W14x605 & W14x605 \\
GB & W16x40 & W18x46 & W18x46 \\
LNK1 & W14x159 & W14x145 & A \\
LNK2 & W14x159 & W14x145 & A \\
LNK3 & W14x159 & W14x145 & A \\
LNK4 & W14x159 & W10x45 & B \\
LNK5 & W14x159 & W10x45 & B \\
\hline
\end{tabular}

Table 8.7: Third model link dimensions (in)

\begin{tabular}{cccccc}
\hline \multicolumn{4}{c}{ 4-story LCF } & \multicolumn{4}{c}{ sample building } \\
\hline Links & $h_{w}$ & $t_{w}$ & $b_{f}$ & $t_{f}$ \\
\hline $\mathrm{A}$ & 10 & 0.5 & 17.4 & 1.5 \\
$\mathrm{~B}$ & 6 & 0.5 & 17.4 & 1.0 \\
\hline
\end{tabular}

The same elastic analysis that was performed for the first model was also conducted for both second and third models. Table (8.8) indicates that the second model satisfied the drift limit, but now the difference between $\Delta_{a}$ and $\delta_{x}$, at roof level, was reduced to about $7 \%$. First, second and third floor levels differences were $6 \%, 14 \%$ and $24 \%$, respectively. The more the difference is close to zero, more the structure tends to be optimal. Unfortunately, wide flange sections are not versatile enough to minimize those differences. 
Table 8.8: Second model drift analysis for the LCF sample building.

\begin{tabular}{rcccccc}
\hline & $\mathrm{h}$ & $\delta_{x e}$ & $\Delta_{x e}$ & $\delta_{x}$ & $\Delta_{a}$ & $\%$ \\
\hline Roof & 156 & 2.52 & 0.66 & 3.63 & 3.9 & 7 \\
Floor 3 & 156 & 1.86 & 0.67 & 3.69 & 3.9 & 6 \\
Floor 2 & 156 & 1.19 & 0.62 & 3.41 & 3.9 & 14 \\
Floor 1 & 156 & 0.57 & 0.57 & 3.14 & 3.9 & 24 \\
\hline
\end{tabular}

Built-up sections become practical to control the strength of the shear link while controlling the drift of the system. They also offer flexibility in regards to thickness of the flange and web, and also the depth of the section to desired values. The difference between $\Delta_{a}$ and $\delta_{x}$ was analyzed for the third model which consisted of built-up sections along the height of the LCF building. As expected, the third model not only satisfied the drift limit, but also reduced the difference between the inter-story drifts to less than $5 \%$ in all floors, as indicated in Table (8.9).

Table 8.9: Third model drift analysis for the LCF sample building.

\begin{tabular}{rcccccc}
\hline & $\mathrm{h}$ & $\delta_{x e}$ & $\Delta_{x e}$ & $\delta_{x}$ & $\Delta_{a}$ & $\%$ \\
\hline Roof & 156 & 2.76 & 0.67 & 3.71 & 3.9 & 5 \\
Floor 3 & 156 & 2.09 & 0.70 & 3.85 & 3.9 & 1 \\
Floor 2 & 156 & 1.39 & 0.71 & 3.90 & 3.9 & 0 \\
Floor 1 & 156 & 0.68 & 0.68 & 3.74 & 3.9 & 4 \\
\hline
\end{tabular}




\section{Pushover Analysis}

Nonlinear static pushover analyses were conducted for LCF behavior evaluation purposes. The response for three numerical models, ranging from a preliminary LCF structure which used the same shear links along the height of the building to an optimized model that incorporated built-up sections as shear links, is evaluated.

The effect of modeling assumptions is highlighted using nonlinear pushover curves for the different models. First yield link and first yield gravity beam, obtained by subjecting the different models to a target displacement, are compared do evaluate the influences of different types of LCF structures toward to rapid return to occupancy. Figure (8.11) shows the normalized base shear versus the normalized drift response for the different models.

This comparison forms the basis for the selection of a representative model to be used in a more refined dynamic time-history analysis, which is not the scope of this research. However, pushover analysis provide information in regards to inelastic deformation of the structure which cannot be studied in detail from a time- history analysis perspective. The nonlinear static pushover analysis procedure has been shown to provide a reasonable estimate of the deformation response for LCF structures. Differences on performance levels for the different models are discussed in the next section. 


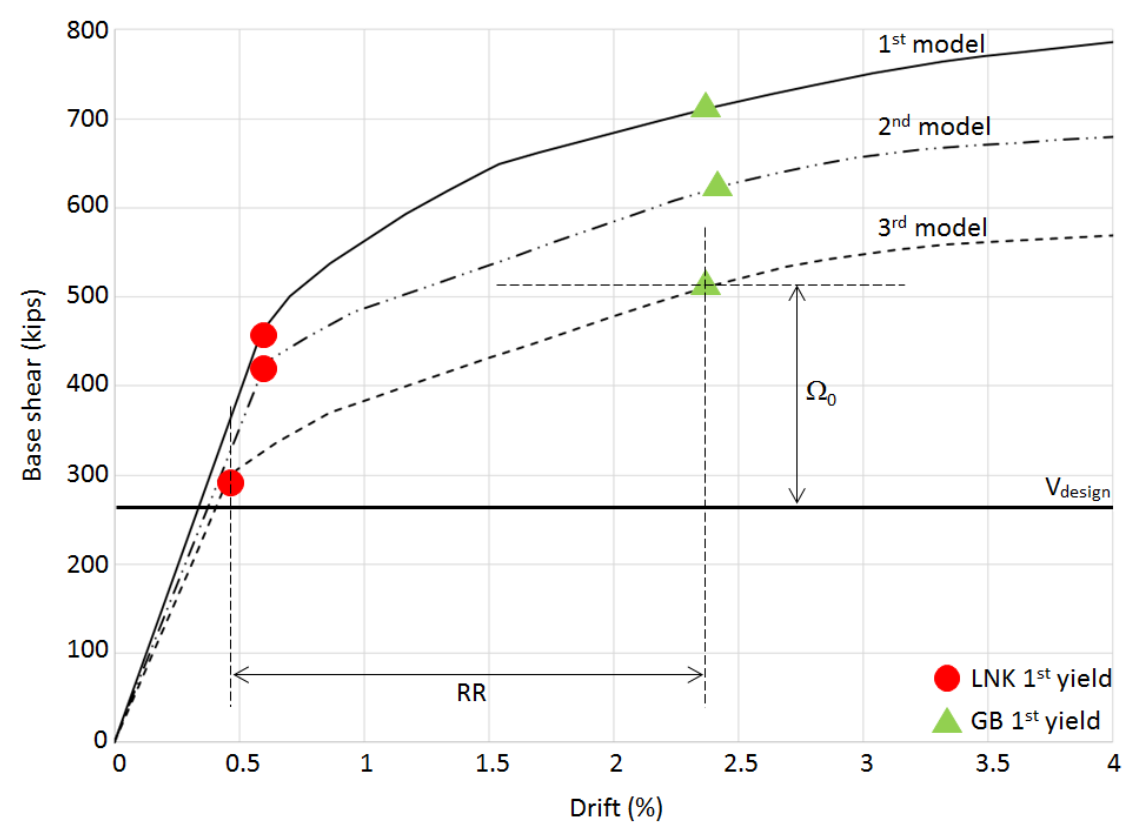

Figure 8.11: Pushover curves for the LCF sample building.

\section{Performance Levels}

The main goal of the LCF system is to achieve performance levels that could result in post-event rapid return to occupancy. This is only possible due to ductile steel shear links that limit the demand on the rest of the structure and also to dissipate energy.

Under extreme lateral earthquake loads, the relative displacements of the linked columns engage the links which are designed to yield in shear to dissipate energy, control drift and limit the forces transferred to the surrounding structural members.

The pushover curves for the LCF sample building shown in Figure (8.11) indicate that there were similarities and differences in the response of the three 
different models. The elastic stiffness of all models is almost identical, indicating that IO performance level, where the system remains linearly elastic, is almost the same. The range of the RR performance level is almost identical as well. However, the first yield link for the third model had a base shear $(V=294$ kips $)$ closer to the design base shear $\left(V_{\text {design }}=260\right.$ kips $)$ when compared to the first and second models, $V=460$ kips and $V=420$ kips, respectively. Also, the first yield of the gravity beam for the third model had a base shear of $V=511$ kips whereas the base shear for the first and second models were, $V=712$ kips and $V=625$ kips, respectively, indicating a foundation demand reduction from the first to the third model. This shed light on the system overstrength factor, $\Omega_{0}$, that varied from 1.96 to 2.74 , a difference about $40 \%$, indicating a controlled flexibility on this seismic parameter. Table (8.10) indicates the performance parameters for the LCF sample building using different models. 
Table 8.10: Performance parameters for the LCF sample building.

\begin{tabular}{cccc}
\hline Parameters & $1^{s t}$ model & $2^{\text {nd }}$ model & $3^{\text {rd }}$ model \\
\hline$\Delta_{L C}$ & $3.66 \mathrm{in}$ & $3.71 \mathrm{in}$ & $2.83 \mathrm{in}$ \\
$V_{L C}$ & $460 \mathrm{kips}$ & $420 \mathrm{kips}$ & $294 \mathrm{kips}$ \\
$\%_{L C}$ & $0.59 \%$ & $0.59 \%$ & $0.45 \%$ \\
$\Delta_{M F}$ & $14.88 \mathrm{in}$ & $15.27 \mathrm{in}$ & $14.81 \mathrm{in}$ \\
$V_{M F}$ & $712 \mathrm{kips}$ & $625 \mathrm{kips}$ & $511 \mathrm{kips}$ \\
$\%_{M F}$ & $2.38 \%$ & $2.44 \%$ & $2.37 \%$ \\
$\mathrm{RR}$ & $1.79 \%$ & $1.85 \%$ & $1.92 \%$ \\
$\Omega_{0}$ & 2.74 & 2.40 & 1.96 \\
$V_{L C} / V_{M F}$ & 0.65 & 0.67 & 0.58 \\
$K_{L C} / K_{M F}$ & 2.60 & 2.79 & 3.05 \\
$\Delta_{L C} / \Delta_{M F}$ & 0.25 & 0.24 & 0.19 \\
$V_{L C} / V_{\text {design }}$ & 1.77 & 1.61 & 1.13 \\
$\mathrm{FR}$ & $1835 \mathrm{kips}$ & $1245 \mathrm{kips}$ & $930 \mathrm{kips}$ \\
\hline
\end{tabular}

Lateral performance ratio parameters were also calculated and compared. Base shear ratios, $V_{L C} / V_{M F}$, and stiffness ratios, $K_{L C} / K_{M F}$, were almost the same, leading to a relative displacement ratio, $\Delta_{L C} / \Delta_{M F}$, between 0.19 and 0.25 which indicates a potential system for rapid return to occupancy. Should the deformation ratio greater than unity, increase the stiffness of links and linked columns before increasing beams. Ratio between first yield link and base shear design, $V_{L C} / V_{\text {design }}$, and foundation axial reactions on LC2 were also determined. 
The first model presented a ratio of, $V_{L C} / V_{\text {design }}=1.77$, and a foundation reaction of $F R=1835$ kips, whereas the third model presented a ratio of, $V_{L C} / V_{\text {design }}=1.13$, and a foundation reaction of $F R=930$ kips, also indicating a significant foundation demand reduction. LCF structures present the advantage of controlling the demand on the foundation elements, just changing the link layout. As expected, all three models presented a reasonable RR level of performance and none of them exhibit column yielding. All in all, the third model had a better performance and is more economical when compared to the other two models. 


\section{Chapter 9}

\section{Seismic Performance Assessment}

\subsection{Introduction}

This chapter presents the results of a structural seismic performance assessment using ASCE/SEI 41 (2013) procedures utilizing LCF as the lateral force resisting system. The main goal of this assessment was to find out if LCF buildings provide consistent levels of performance according to this relatively new standard (not a code) for seismic evaluation and retrofit of existing buildings. ASCE/SEI 41 (2013) replaces the seismic evaluation ASCE/SEI 31 (2003) and the seismic rehabilitation ASCE/SEI 41 (2006) design standards. In order to seismic evaluate a building, target building performance levels shall be selected, such as Imediate Occupancy (IO) Building Performance Level, which is a seismic hazard with a 50\% probability of exceedance in 50 years, Life Safety (LS) Building Performance Level, which is a seismic hazard with a $20 \%$ probability

of exceedance in 50 years and Collapse Prevention (CP) Building Performance Level, which is a seismic hazard with a $5 \%$ probability of exceedance in 50 
years. The seismic performance assessment was conducted using a linear analysis procedure and assessment calculations for beams, shear links and columns are presented.

\subsection{Analysis Procedure Selection}

There are four types of analysis stated in ASCE/SEI 41 (2013) that could potentially be used for both the evaluation of an existing building and the design of retrofit measures, as follows: 1) Linear Static Procedure (LSP), 2) Linear Dynamic Procedure (LDP), 3) Nonlinear Static Procedure (NSP) and 4) Nonlinear Dynamic Procedure (NDP). Each of those methods have their own requirements and limitations.

In general, static procedures are appropriate where the first mode of vibration dominates the response of the structure, whereas dynamic procedures are required for buildings that have significant contributions of higher modes and for buildings with torsional irregularities. Linear analysis obeys Hooke's law and has a linear stress-strain relationship, whereas nonlinear analysis has an inelastic response.

LCF systems tend to be short-to-mid height buildings and for the cases presented, in this research, the buildings do not have torsional irregularities. For these reasons, the LSP seems to be appropriate. However, the magnitude and distribution of inelastic demands for existing components shall be defined by demand-capacity ratios (DCRs) and calculated according to Equation (9.1). 


$$
D C R=\frac{Q_{U D}}{Q_{C E}}
$$

where $Q_{U D}$ is the force caused by gravity loads and earthquake forces and $Q_{C E}$ is the expected strength of the component. If a component DCR is greater than 3.0, then linear procedures are not applicable and shall not be used. Should DCR values remain below the threshold, an acceptance criteria must be establish in terms of the various response quantities obtained from the analysis.

If the LSP is selected, forces and deformations in components shall be calculated for a pseudo seismic force calculated in accordance with ASCE/SEI 41 (2013). This pseudo seismic force is similar to the base shear used in the equivalent lateral force procedure defined in ASCE/SEI 7 (2010). For a LCF building, the LSP can be performed by following the flowchart given in Figure (9.1).

\subsection{Linear Static Procedure}

An outline of the step-by-step Linear Static Procedure applied to a LCF building is presented. Even though the procedure is so called linear static, nonlinear and modal analyses should be performed prior using this method, in order to find parameters to calculate pseudo seismic forces distributed throughout the building. The idea behind the pseudo seismic force, $V$, is when applied to the structure, it results in displacement amplitudes approximating maximum 


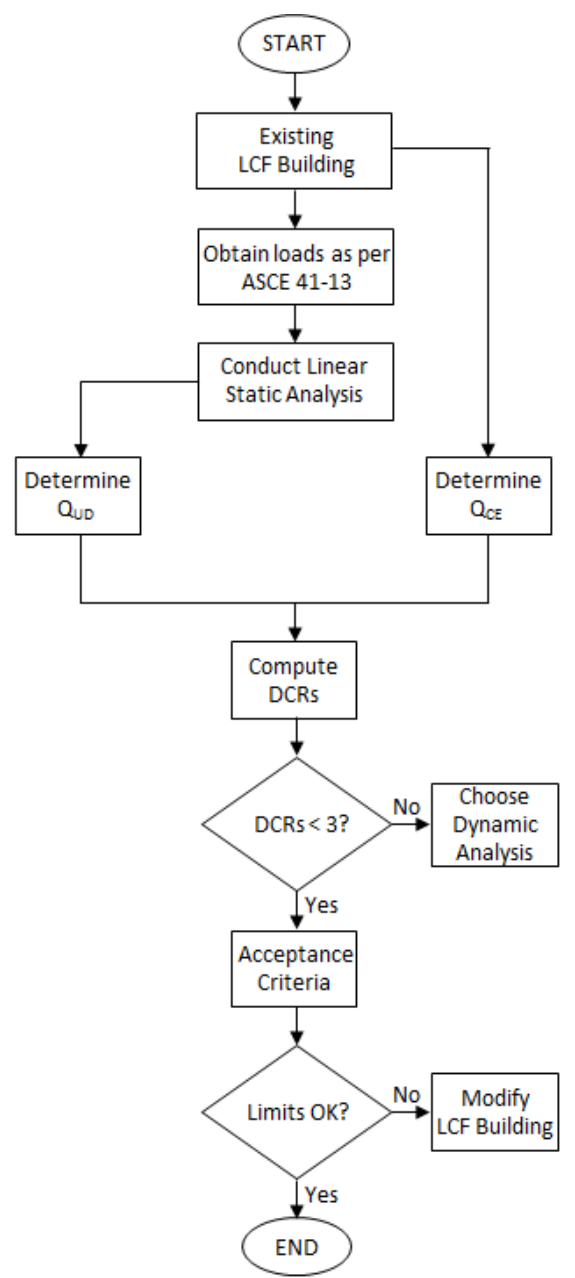

Figure 9.1: Flowchart for the LCF linear static procedure.

displacements expected during the selected target performance levels. The stepby-step is presented as follows:

1. Define the LCF building location. Once the building site is selected, several parameters should be established. The United States Geological Survey (USGS) - US Seismic Design Maps Application - provides the values of the response acceleration parameters, $S_{S}$ and $S_{1}$. For the given site class and the values of $S_{S}$ and $S_{1}$, the site coefficient parameters, $F_{a}$ and 
$F_{v}$, are determined. Site class factor, $a$, varies from 130 (site class A or B) to 60 (site class $\mathrm{D}, \mathrm{E}$ or $\mathrm{F}$ ). Also, $\beta$, which is the effective viscous damping ratio is chosen.

2. Typically, a 2-5\% damping ratio is implicit in the code-specified earthquake forces and design spectrum. The damping factor, $B_{1}$, is calculated according to Equation (9.2).

$$
B_{1}=\frac{4}{5.6-\ln (100 \beta)}
$$

3. Determine the design spectral response acceleration parameters, $S_{X S}$ and $S_{X 1}$, according to the following set of Equations (9.3):

$$
\begin{gathered}
S_{X S}=F_{a} S_{S} \\
S_{X 1}=F_{v} S_{1}
\end{gathered}
$$

4. Once $S_{X S}$ and $S_{X 1}$ are established, a general horizontal response spectrum shall be develop as shown in Figure (9.2).

Determine values for $T_{0}$ and $T_{S}$ using Equations (9.4) and (9.5), respectively.

$$
\begin{gathered}
T_{0}=0.2 T_{S} \\
T_{S}=\frac{S_{X 1}}{S_{X S}}
\end{gathered}
$$




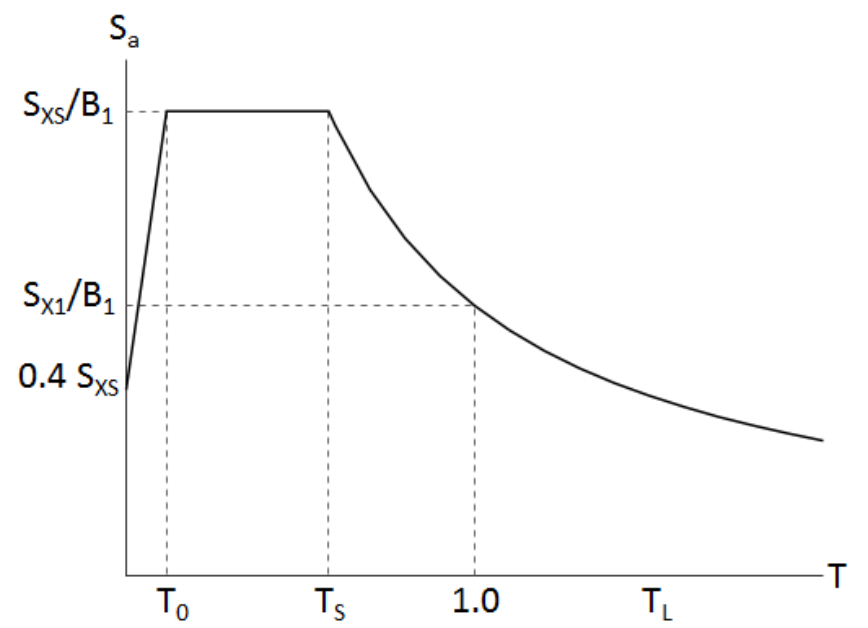

Figure 9.2: General Horizontal Response Spectrum.

5. Determine the effective seismic weight of the building, $W$, and the effective mass factor, $C_{m}$, while performing a LCF modal analysis in order to obtain the period of the structure, $T$.

6. Perform a LCF nonlinear static analysis and determine the yield strength of the building, $V_{y}$.

7. Determine the spectral response acceleration, $S_{a}$, as follows:

For the short period region, $0<T<T_{0}$, known as acceleration sensitive region, $S_{a}$ is given by

$$
S_{a}=\left[\left(\frac{5}{B_{1}}-2\right) \frac{T}{T_{S}}+0.4\right]
$$

For the intermediate period region between points $T_{0}$ and $T_{S}$, known as 
velocity sensitive region, $S_{a}$ is calculated according to

$$
S_{a}=\frac{S_{X S}}{B_{1}}
$$

Finally, the region period region between points $T_{S}$ and $T_{L}$ is called displacement sensitive region because structural response is related most directly to ground displacement. LCF tends to be drift controlled structures. Therefore, for this region, $S_{a}$ becomes

$$
S_{a}=\frac{S_{X 1}}{B_{1} T}
$$

8. By now a pushover analysis was already performed. Determine the ratio of elastic strength demand to yield strength coefficient, $\mu_{\text {strength }}$, in function of yield strength of the building and mass parameters. $\mu_{\text {strength }}$ shall be calculated in accordance with:

$$
\mu_{\text {strength }}=\frac{S_{a}}{\left(V_{y} / W\right)} C_{m}
$$

9. Determine the modification factors $C_{1}$ and $C_{2}$, where $C_{1}$ is the modification factor to relate expected maximum inelastic displacements to displacements calculated for linear elastic response. For fundamental periods less than $0.2 \mathrm{sec}, C_{1}$ need not be taken as greater than the value at $T=0.2 \mathrm{sec}$. For fundamental periods greater that $1.0 \mathrm{sec}, C_{1}=1.0 . C_{2}$ is 223 
the modification factor to represent the effect of pinched hysteresis shape, cyclic stiffness degradation, and strength deterioration on maximum displacement response. For fundamental periods greater than $0.7 \mathrm{sec}, C_{2}=$ 1.0. $C_{1}$ and $C_{2}$ are given by the following Equations, respectively.

$$
\begin{gathered}
C_{1}=1+\frac{\mu_{\text {strength }}-1}{a T^{2}} \\
C_{2}=1+\frac{1}{800}\left(\frac{\mu_{\text {strength }}-1}{T}\right)^{2}
\end{gathered}
$$

10. Finally, determine the pseudo lateral force according to Equation (9.12).

$$
V=C_{1} C_{2} C_{m} S_{a} W
$$

Once the pseudo lateral force is obtained, the lateral forces are distributed throughout the building in a similar way as the equivalent lateral force procedure. The pseudo seismic force analysis for the LSP of a typical LCF system can be performed by following the flowchart given in Figure (9.3) and its application is illustrated in Section (9.7). 


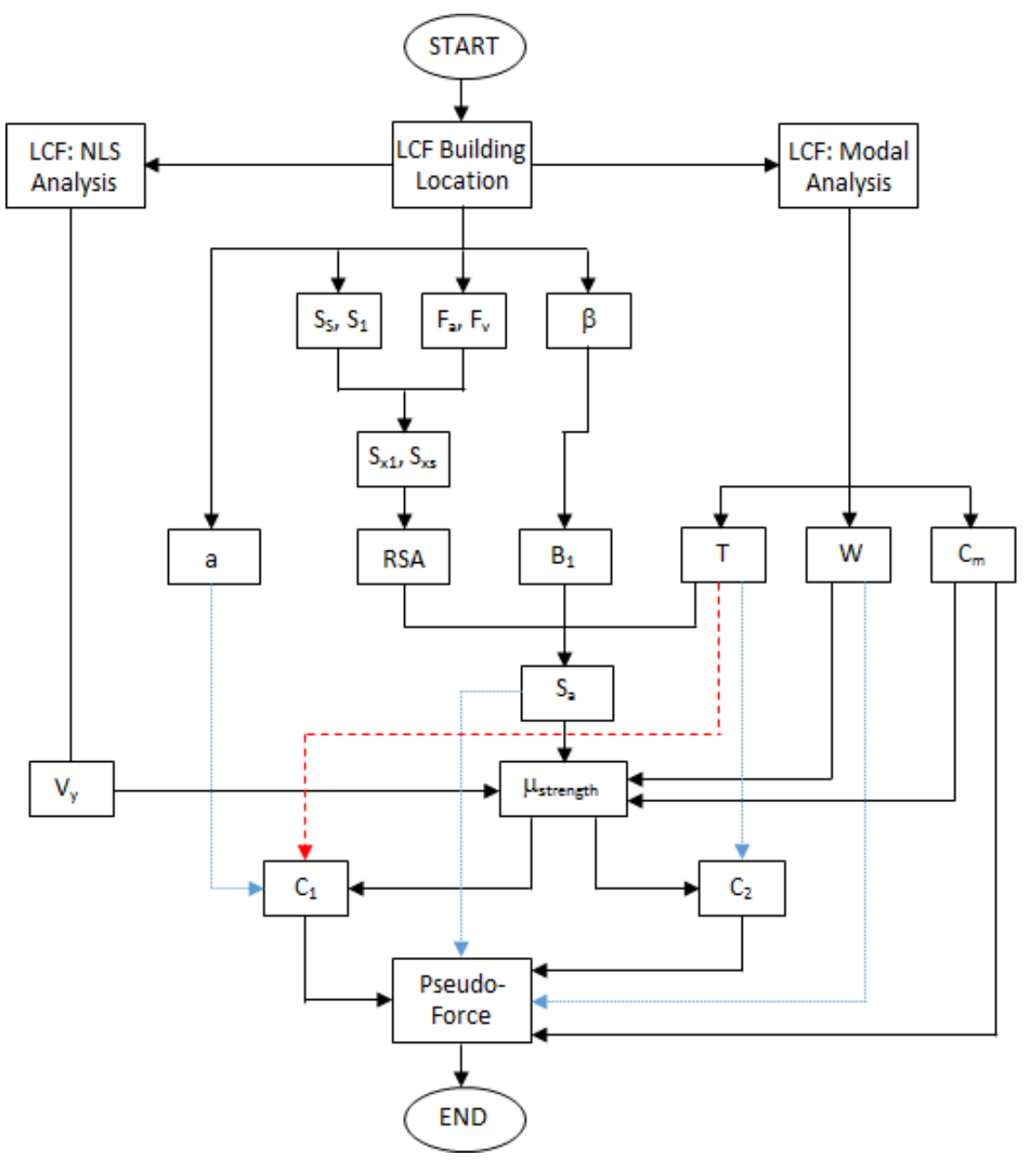

Figure 9.3: LCF pseudo seismic force flowchart for linear static procedure.

\subsection{Load Combination}

For the linear static procedure, the following actions caused by gravity loads shall be considered for combination with actions caused by seismic forces: dead and live loads. The effects of gravity loads and seismic forces are additive. The component gravity load combination used in the analysis for the LCF buildings 
is given by Equation (9.13).

$$
Q_{G}=1.1\left(Q_{D}+Q_{L}\right)
$$

where $Q_{G}$ is the action caused by gravity loads, $Q_{D}$ is the action caused by dead loads and $Q_{L}$ is the action caused by live loads, equal to $25 \%$ of the unreduced live load. Therefore, the coefficients for the load combination are as follows: $Q_{D}=1.1, Q_{L}=0.28$ and $Q_{E}=1.0$. If the actions of gravity loads and seismic forces are counteracting, $Q_{G}$, shall be obtained according to Equation (9.14).

$$
Q_{G}=0.9 Q_{D}
$$

\subsection{Strength of LCF components}

The capacity of LCF components to be used in Equation (9.1) is defined by its strength according to the limit states for each structural member. This section summarizes the calculations that were used in the LCF design process for beams, shear links and columns.

\subsubsection{Beams}

The expected flexural strength $\left(Q_{C E}\right)$ of flexural deformation-controlled members shall be the lowest value obtained for the limit states of local flange buckling, local web buckling, moment yielding, lateral-torsional buckling, or shear 
yielding. The procedure for computation of expected flexural strength for compact wide-flange sections bent about the $x$ axis is summarized as follows.

1. Check local flange buckling.

$$
\frac{b_{f}}{2_{t f}} \leq 0.38 \sqrt{\frac{E}{F_{y}}}
$$

where $b_{f}$ is the flange width, $t_{f}$ is the thickness of the flange, $E$ is the modulus of elasticity, and $F_{y}$ is the yield stress.

2. Check local web buckling.

$$
\frac{h}{t_{w}} \leq 2.45 \sqrt{\frac{E}{F_{y}}}
$$

where $h$ is the distance from inside of compression flange to inside of tension flange and $t_{w}$ is $t_{w}$ is the web thickness.

3. Determine the unbraced beam length $\left(L_{b}\right)$, given from the geometry of the LCF building, the largest unbraced beam length for which lateraltorsional buckling will not occur $\left(L_{p}\right)$ and the unbraced beam length at which elastic lateral-torsional buckling will occur $\left(L_{r}\right)$.

$$
L_{p}=1.76 r_{y} \sqrt{\frac{E}{F_{y}}}
$$


where $r_{y}$ is radius of gyration about the $y$ axis.

$$
L_{r}=1.95 r_{t s} \frac{E}{0.7 F_{y}} \sqrt{\frac{J c}{S_{x} h_{0}}} \sqrt{1+\sqrt{1+6.76\left(\frac{0.7 F_{y} S_{x} h_{0}}{E J c}\right)^{2}}}
$$

where $r_{t s}$ is the radius of gyration about the weak axis of a flexural member for a portion of the cross section consisting of the compression flange and one third of the compressed part of the web, $J$ is the polar moment of inertia, $c$ is a constant for critical lateral torsional buckling stress, $S_{x}$ is the elastic section modulus for $x$ axis, and $h_{0}$ is the distance between $\mathrm{W}$-shape flange centroids.

4. For $L_{b} \leq L_{p}$, beam is fully supported and can fail becoming fully plastic. Therefore,

$$
M_{n}=M_{p}
$$

where $M_{n}$ is the nominal bending strength and $M_{p}$ is the plastic moment.

5. For $L_{p}<L_{b} \leq L_{r}$, beam can fail through inelastic lateral-torsional buckling. For this case $M_{n}$ is given by

$$
M_{n}=C_{b}\left[M_{p}-\left(M_{p}-0.7 F_{y} S_{x}\right)\left(\frac{L_{b}-L_{p}}{L_{r}-L_{p}}\right)\right] \leq M_{p}
$$

where $C_{b}$ is the moment gradient factor for lateral-torsional buckling strength. 
6. For $L_{b}>L_{r}$, beam can fail through elastic lateral-torsional buckling. Thus,

$$
M_{n}=F_{c r} S_{x} \leq M_{p}
$$

where $F_{c r}$ is the critical bending stress and is given by

$$
F_{c r}=\frac{C_{b} \pi^{2} E}{\left(\frac{L_{b}}{r_{t s}}\right)^{2}} \sqrt{1+0.078 \frac{J c}{S_{x} h_{0}}\left(\frac{L_{b}}{r_{t s}}\right)^{2}}
$$

7. Check shear

$$
V_{n}=0.6 F_{y} A_{w} C_{v}
$$

where $V_{n}$ is the nominal shear strength, $A_{w}$ is the nominal area of the web and $C_{v}$ is the ratio of critical web stress to shear yield stress.

\subsubsection{Replaceable Links}

Replaceable links shall be considered as deformation-controlled actions. The procedure for computation of the expected shear strength is summarized as follows.

1. Determine the plastic moment $\left(M_{p}\right)$

$$
M_{p}=F_{y} Z_{x}
$$

where $Z_{x}$ is the plastic section modulus for $x$ axis. 
2. Determine the shear force for shear yielding in the web $\left(V_{p}\right)$

$$
V_{p}=0.6 F_{y} A_{w}
$$

3. In order to be a shear link, check if the length of the link $(e)$ is limited as follows:

$$
e \leq \frac{1.6 M_{p}}{V_{p}}
$$

4. Check if the link strength is governed by the shear strength of the unstiffened web given by

$$
\frac{h}{t_{w}} \leq \frac{418}{\sqrt{F_{y}}}
$$

5. Determine $V_{C E}$ in accordance with Equation (9.28).

$$
Q_{C E}=V_{C E}=0.6 F_{y e} A_{w}
$$

where $V_{C E}$ is the expected shear strength and $F_{y e}$ is the expected yield strength of the material.

\subsubsection{Columns}

The lower-bound strength $\left(Q_{C L}\right)$ of steel columns under axial compression shall be the lowest value obtained for the limit states of local flange buckling, local 
web buckling or column buckling. The procedure for computation of the lowerbound strength for nonslender element compression members is summarized as follows.

1. Check local flange buckling.

$$
\frac{b_{f}}{2_{t f}} \leq 0.56 \sqrt{\frac{E}{F_{y}}}
$$

2. Check local web buckling.

$$
\frac{h}{t_{w}} \leq 1.49 \sqrt{\frac{E}{F_{y}}}
$$

3. Determine the effective slenderness ratio.

$$
\frac{K L}{r_{y}} \leq 200
$$

where $K$ is the effective length factor for compression members and $L$ is the member length.

4. Calculate Euler buckling stress $\left(F_{e}\right)$

$$
F_{e}=\frac{\pi^{2} E}{\left(\frac{K L}{r_{y}}\right)^{2}}
$$

5. In order to calculate the critical compressive stress $\left(F_{c r}\right)$ according to 
Equation (9.47), check the following ratio

$$
\begin{gathered}
\frac{K L}{r_{y}} \leq 4.71 \sqrt{\frac{E}{F_{y}}} \\
F_{c r}=\left(0.658^{\frac{F_{y}}{F_{e}}}\right) F_{y}
\end{gathered}
$$

Otherwise, $\left(F_{c r}\right)$ is given according to Equation (9.35)

$$
F_{c r}=0.877 F_{e}
$$

6. Determine the nominal compressive strength $\left(P_{n}\right)$

$$
P_{n}=F_{c r} A_{g}
$$

where $A_{g}$ is the gross area of the column.

For the structural members that are subjected to axial tensile forces, the expected axial strength of a column in tension, $T_{C E}$, shall be computed in accordance with Equation (9.37):

$$
T_{C E}=A_{g} F_{y}
$$




\subsection{Acceptance Criteria}

The performance of a LCF building is assessed based on demand-capacity ratio values. When the ratio is less than or equal to 1.0, then the structural component is expected to behave elastically at a target performance level. On the other hand, an inelastic response to the earthquake ground shaking is achieved when the ratio is greater than 1.0.

Before selecting the corresponding acceptance criteria, LCF structural members shall be classified as primary or secondary components, where the primary component is the one which is required to resist seismic forces and accommodate deformations for the structure to achieve a selected performance level. Also, LCF structural members shall be classified as deformation or force controlled depending on its actions (internal forces, deformations, drift, stiffness). For the LSP, flexure on beams and shear on links are considered deformationcontrolled actions, whereas axial load on columns could be either deformation or force-controlled action. For a LCF building, the acceptance criteria can be performed by following the flowchart given in Figure (9.4).

The acceptance criteria for flexure beam and shear on a short link are given by Equations (9.38) and (9.39), respectively.

$$
D C R=\frac{M_{U D}}{\kappa m M_{C E}}
$$

where $M_{U D}$ is the flexure demand acting on the beam, $M_{C E}$ is the flexure beam 


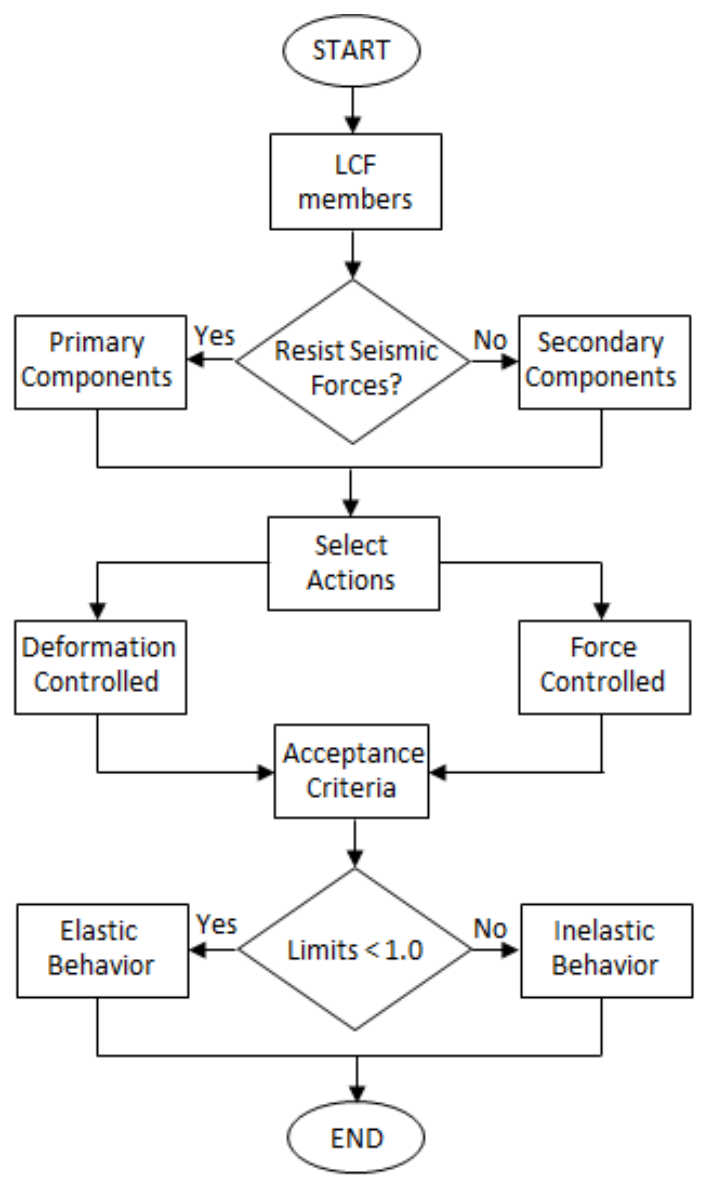

Figure 9.4: LCF acceptance criteria flowchart.

strength, $\kappa$ is a knowledge factor used to reduce component strength based on the level of knowledge obtained for individual components during data collection, and $m$ is the component demand modification factor to account for expected ductility associated with this action at the selected structural performance level.

$$
D C R=\frac{V_{U D}}{\kappa m V_{C E}}
$$

$V_{U D}$ is the shear demand acting on the short link and $V_{C E}$ is the strength of the short link. 
The acceptance criteria for steel columns under combined axial compression and bending stresses could be considered either deformation or force-controlled actions, depending on the ratio of $\frac{P_{U F}}{P_{U L}}$, where $P_{U F}$ is the compression demand acting on the column and $P_{U L}$ is the column strength.

For $0.2<\frac{P_{U F}}{P_{U L}}<0.5$, the acceptance criteria shall be evaluated by Equation (9.40).

$$
\frac{P_{U F}}{P_{C L}}+\frac{8}{9}\left[\frac{M_{x}}{\kappa m M_{C E}}\right] \leq 1.0
$$

where $M_{x}$ is the moment demand in the member for the x-axis.

For $\frac{P_{U F}}{P_{U L}}<0.2$, the acceptance criteria shall be computed by Equation (9.41).

$$
\frac{P_{U F}}{2 P_{C L}}+\frac{M_{x}}{\kappa m M_{C E}} \leq 1.0
$$

And, finally, for $\frac{P_{U F}}{P_{U L}}>0.5$, the acceptance criteria shall be considered force controlled for both axial loads and flexure and is determined by Equation (9.42).

$$
\frac{P_{U F}}{P_{C L}}+\frac{M_{x}}{M_{C E}} \leq 1.0
$$

Steel columns under combined axial tension and bending stresses shall be considered deformation controlled and evaluated by Equation (9.43).

$$
\frac{T}{\kappa m_{t} T_{C E}}+\frac{M_{x}}{\kappa m_{x} M_{C E}} \leq 1.0
$$


where $T$ is the tensile load demand on column, $m_{t}$ is the value of $m$ for the column in tension and $m_{x}$ is the value of $m$ for the column bending about the x-axis. Equations (9.40) through (9.43) play an important role on the assessment of the linked-columns, since the columns would perform either on the compression or tension sides.

\subsection{Assessment Example}

This section presents an example detailing the linear static assessment calculations for the following primary structural members: gravity beams, linked columns and shear links. The LCF system analyzed was the 2-story building (LCF-5L), experimentally tested, with its specifications given in Chapter (4). After several analyses and design iterations, prior to the LCF experimental test, the final member sizes for the LCF 2-story building are shown in Figure (9.5). GB-SA, LC-SA and LNK-SA are selected for hand calculations, whereas the rest of the building labeled as shown in Figure (9.6) would be evaluated afterwards. A LSP was performed on the LCF 2-story building and the corresponding seismic hazard parameters are given in Table (9.1). 


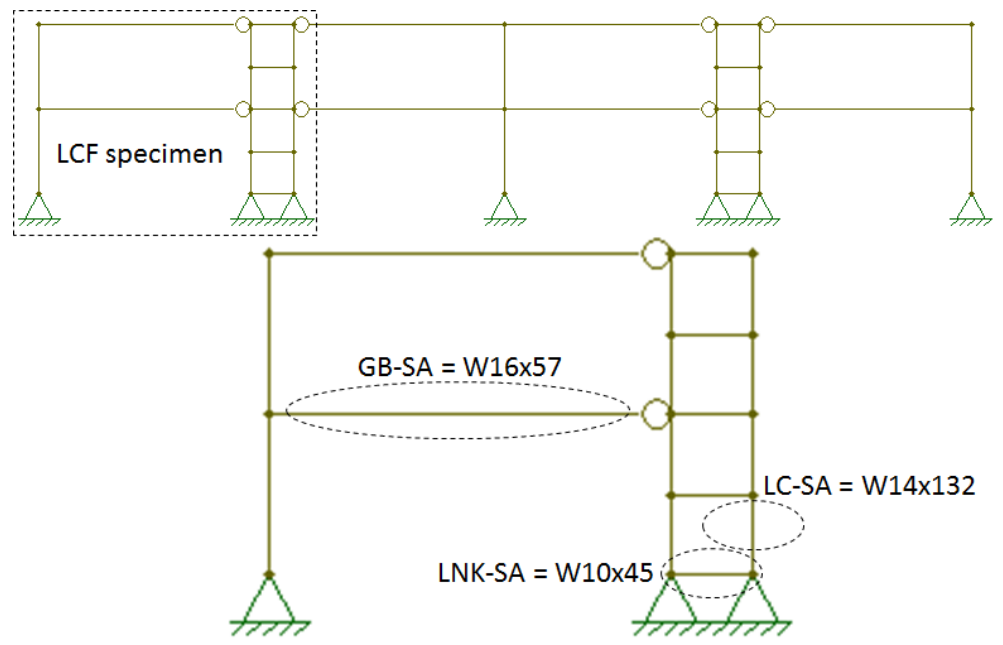

Figure 9.5: Seismic assessment for structural members on the LCF 2-story building.

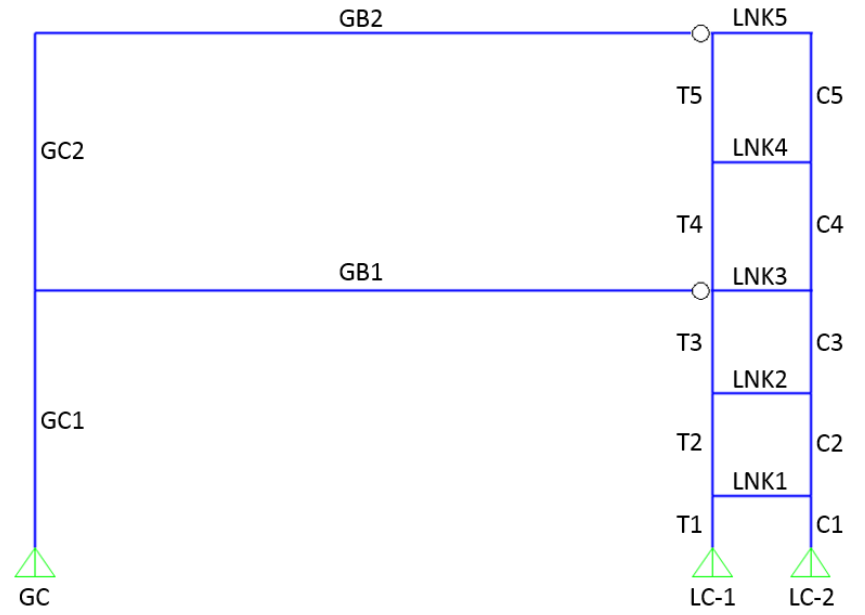

Figure 9.6: LCF 2-story building labeling for seismic assessment. 
Table 9.1: Seismic design parameters for the LSP on the LCF 2-story building.

\begin{tabular}{|c|c|}
\hline Parameter & Value \\
\hline $\mathrm{S}_{\mathrm{S}}$ & $0.498 \mathrm{~g}$ \\
\hline $\mathrm{S}_{1}$ & $1.287 \mathrm{~g}$ \\
\hline $\mathrm{F}_{\mathrm{a}}$ & 1.0 \\
\hline $\mathrm{F}_{\mathrm{v}}$ & 1.5 \\
\hline $\mathrm{a}$ & 60 \\
\hline$\beta$ & $2.0 \%$ \\
\hline $\mathrm{B}_{1}$ & 0.82 \\
\hline $\mathrm{S}_{\mathrm{XS}}$ & $0.75 \mathrm{~g}$ \\
\hline $\mathrm{S}_{\mathrm{X} 1}$ & $1.287 \mathrm{~g}$ \\
\hline $\mathrm{T}_{0}$ & $0.12 \mathrm{sec}$ \\
\hline $\mathrm{T}_{\mathrm{S}}$ & $0.58 \mathrm{sec}$ \\
\hline $\mathrm{T}$ & $0.80 \mathrm{sec}$ \\
\hline $\mathrm{W}$ & 2,857 kips \\
\hline $\mathrm{C}_{\mathrm{m}}$ & 1.0 \\
\hline $\mathrm{V}_{\mathrm{y}}$ & 210 kips \\
\hline $\mathrm{S}_{\mathrm{a}}$ & $1.14 \mathrm{~g}$ \\
\hline$\mu_{\text {strength }}$ & 7.8 \\
\hline $\mathrm{C}_{1}$ & 1.18 \\
\hline $\mathrm{C}_{2}$ & 1.0 \\
\hline $\mathrm{V}$ & 1922 kips \\
\hline
\end{tabular}




\subsubsection{Beams}

\subsubsection{Flexure Demand}

Flexure actions in the gravity beam are considered deformation controlled and shall be calculated according to Equation (9.44)

$$
Q_{U D}=Q_{G}+Q_{E}
$$

where $Q_{U D}$ is the deformation controlled action caused by gravity loads and earthquake forces, $Q_{G}$ is the action caused by gravity loads and $Q_{E}$ is the action caused by the response to the selected seismic hazard level. Thus, the maximum moment reported on GB-SA was:

$$
M_{U D}=2,959 \text { kips.in }
$$

\subsubsection{Flexure Strength}

For the determination of the flexure strength of component GB-SA, the procedure described in Section (9.5.1) was used

1. Check local flange buckling.

$$
\frac{b_{f}}{2_{t f}} \leq 0.38 \sqrt{\frac{E}{F_{y}}}=4.98 \leq 0.38 \sqrt{\frac{29000}{50}}=9.15 \Rightarrow \checkmark
$$


2. Check local web buckling.

$$
\frac{h}{t_{w}} \leq 2.45 \sqrt{\frac{E}{F_{y}}}=33 \leq 2.45 \sqrt{\frac{29000}{50}}=59 \Rightarrow \checkmark
$$

3. Determine $L_{b}, L_{p}$ and $L_{r}$.

$$
\begin{gathered}
L_{b}=288 \mathrm{in} \\
L_{p}=1.76 r_{y} \sqrt{\frac{E}{F_{y}}}=1.76(1.60) \sqrt{\frac{29000}{50}}=68 \mathrm{in} \\
L_{r}=1.95 r_{t s} \frac{E}{0.7 F_{y}} \sqrt{\frac{J c}{S_{x} h_{0}}} \sqrt{1+\sqrt{1+6.76\left(\frac{0.7 F_{y} S_{x} h_{0}}{E J c}\right)^{2}}} \\
L_{r}=220 \mathrm{in}
\end{gathered}
$$

4. Since $L_{b}>L_{r}$, GB-SA could fail through elastic lateral-torsional buckling. Thus,

$$
M_{p}=Z_{x} F_{y}=(105)(50)=5,250 \text { kips.in }
$$




$$
\begin{aligned}
& F_{c r}=\frac{C_{b} \pi^{2} E}{\left(\frac{L_{b}}{r_{t s}}\right)^{2}} \sqrt{1+0.078 \frac{J c}{S_{x} h_{0}}\left(\frac{L_{b}}{r_{t s}}\right)^{2}} \\
& F_{c r}=\frac{(1.14) \pi^{2}(29000)}{\left(\frac{288}{1.92}\right)^{2}} \sqrt{1+0.078 \frac{(2.22)(1.0)}{(92.2)(15.7)}\left(\frac{288}{1.92}\right)^{2}} \\
& F_{c r}=28 k s i \\
& M_{n}=F_{c r} S_{x}=(28)(92.2)=2,582 \text { kips.in } \leq M_{p}=M_{C E} \Rightarrow \checkmark
\end{aligned}
$$

5. Determine shear

$$
V_{n}=0.6 F_{y} A_{w} C_{v}=0.6(50)(6.44)(1.0)=193 \text { kips }
$$

\subsubsection{Acceptance Criteria Check}

The $m$ factor for GB-SA defined as primary component is taken from ASCE/SEI 41 (2013) as per the acceptance criteria for linear procedures - structural steel components. In order to choose the proper $m$ factor, the following conditions must be satisfied:

$$
\frac{b_{f}}{2_{t f}} \leq \frac{52}{\sqrt{F_{y}}}=4.98 \leq \frac{52}{\sqrt{50}}=7.35 \Rightarrow \checkmark
$$




$$
\frac{h}{t_{w}} \leq \frac{418}{\sqrt{F_{y}}}=33 \leq \frac{418}{\sqrt{50}}=59.11 \Rightarrow \checkmark
$$

Therefore, the $m$ factor for the CP performance level is $m=8$. Since the 2-story LCF building has testing records that are available to substantiate the design drawings, the knowledge factor, $\kappa$, is considered to be 1.0.

The acceptance criteria check for GB-SA is given below, which satisfies the $\mathrm{CP}$ acceptance criteria.

$$
D C R=\frac{M_{U D}}{\kappa m M_{C E}}=\frac{2,959}{(1)(8)(2,582)}=0.14<1.0 \Rightarrow \checkmark
$$

Table (9.2) indicates the DCR values for gravity beams shown in Figure (9.6)

Table 9.2: DCR values for gravity beams on the LCF 2-story building.

\begin{tabular}{ccc}
\hline Beams & $M_{U D}$ & DCR \\
\hline GB 2 & 2621 & 0.13 \\
GB 1 & 2959 & 0.14 \\
\hline
\end{tabular}

\subsubsection{Replaceable Links}

\subsubsection{Shear Demand}

Shear actions in short links are considered deformation controlled and shall be calculated according to Equation (9.45)

$$
V_{U D}=V_{G}+V_{E}
$$


where $V_{U D}$ is the deformation controlled action caused by gravity loads and earthquake forces, $V_{G}$ is the action caused by gravity loads and $V_{E}$ is the action caused by the response to the selected seismic hazard level. Thus, shear reported on LNK-SA was:

$$
V_{U D}=544 k i p s
$$

\subsubsection{Shear Strength}

The flexural and shear strength of LNK-SA is determined as follows:

$$
\begin{aligned}
& M_{C E}=M_{p}=F_{y} Z_{x}=(50)(54.9)=2,745 \text { kips.in } \\
& V_{C E}=V_{p}=0.6 F_{y} A_{w}=0.6(50)(3.10)=93 \text { kips } \\
& e \leq \frac{1.6 M_{p}}{V_{p}}=42 \text { in } \leq \frac{1.6(2,745)}{(93)}=47 \text { in } \Rightarrow \checkmark
\end{aligned}
$$

\subsubsection{Acceptance Criteria Check}

The $m$ factor for LNK-SA defined as primary component is taken from ASCE/SEI 41 (2013) as per the acceptance criteria for linear procedures - structural steel components. In order to choose the proper $m$ factor, the following condition must be satisfied:

$$
e \leq \frac{1.6 M_{p}}{V_{p}} \Rightarrow \checkmark
$$

Therefore, the $m$ factor for the CP performance level is $m=13$.

The acceptance criteria check for LNK-SA is given below, which satisfies the 243 
CP acceptance criteria.

$$
D C R=\frac{V_{U D}}{\kappa m V_{C E}}=\frac{544}{(1)(13)(93)}=0.45<1.0 \Rightarrow \checkmark
$$

Table (9.3) indicates the DCR values for shear links shown in Figure (9.6).

Table 9.3: DCR values for shear links on the LCF 2-story building.

\begin{tabular}{ccc}
\hline Links & $V_{U D}$ & DCR \\
\hline LNK 5 & 226 & 0.19 \\
LNK 4 & 298 & 0.25 \\
LNK 3 & 390 & 0.33 \\
LNK 2 & 458 & 0.38 \\
LNK 1 & 544 & 0.45 \\
\hline
\end{tabular}

\subsubsection{Columns}

\subsubsection{Axial Demand}

The axial force in column LC-SA, $P_{U F}$, is considered to be force-controlled and shall be calculated according to Equation (9.46)

$$
P_{U F}=P_{G}+\frac{P_{E}}{C_{1} C_{2} J}
$$

where $J$ is the force-delivery reduction factor. The value of 2.0 was selected because the building is considered to be in a zone with a high level of seismicity. Thus, the axial force in column is:

$$
P_{U F}=30+\frac{1351}{\left(\begin{array}{c}
1.18)(1)(2) \\
244
\end{array}\right.}=602 \text { kips }
$$




\subsubsection{Axial Strength}

For the determination of the axial strength of component LC-SA, the procedure described in Section (9.5.3) was used

1. Check local flange buckling.

$$
\frac{b_{f}}{2_{t f}} \leq 0.56 \sqrt{\frac{E}{F_{y}}}=7.15 \leq 0.56 \sqrt{\frac{29000}{50}}=13.49 \Rightarrow \checkmark
$$

2. Check local web buckling.

$$
\frac{h}{t_{w}} \leq 1.49 \sqrt{\frac{E}{F_{y}}}=17.7 \leq 1.49 \sqrt{\frac{29000}{50}}=35.88 \Rightarrow \checkmark
$$

3. Determine the effective slenderness ratio.

$$
\frac{K L}{r_{y}} \leq 200=\frac{(1) 44}{3.76}=11.7 \leq 200 \Rightarrow \checkmark
$$

4. Calculate Euler buckling stress $\left(F_{e}\right)$

$$
F_{e}=\frac{\pi^{2} E}{\left(\frac{K L}{r_{y}}\right)^{2}}=\frac{\pi^{2}(29000)}{(11.7)^{2}}=2,088 k s i
$$

5. Calculate the critical compressive stress $\left(F_{c r}\right)$

$$
F_{c r}=\left(0.658^{\frac{F_{y}}{F_{e}}}\right) F_{y}=\left(0.658^{\frac{50}{2,088}}\right)(50)=49.5 k s i
$$


6. Determine the nominal compressive strength $\left(P_{n}\right)$

$$
P_{C L}=P_{n}=F_{c r} A_{g}=49.5(38.8)=1921 \text { kips }
$$

7. Check the ratio between $P_{U F}$ and $P_{C L}$

$$
\frac{P_{U F}}{P_{C L}}=\frac{602}{1921}=0.31<0.50
$$

For steel columns under combined axial compression and bending stresses, where the axial column load is less than $50 \%$ of the lower-bound axial column strength, the column shall be considered deformation controlled for flexural behavior and force controlled for compressive behavior.

\subsubsection{Flexure Demand}

The flexure demand on LC-SA from the controlling load combination was reported as follows:

$$
M_{U F}=6,119 \text { kips.in }
$$

\subsubsection{Flexural Strength}

For the determination of the flexure strength of component LC-SA, the procedure described in Section (9.5.1) was used 
1. Check local flange buckling.

$$
\frac{b_{f}}{2_{t f}} \leq 0.38 \sqrt{\frac{E}{F_{y}}}=7.15 \leq 0.38 \sqrt{\frac{29000}{50}}=9.15 \Rightarrow \checkmark
$$

2. Check local web buckling.

$$
\frac{h}{t_{w}} \leq 2.45 \sqrt{\frac{E}{F_{y}}}=17.7 \leq 2.45 \sqrt{\frac{29000}{50}}=59 \Rightarrow \checkmark
$$

3. Determine $L_{b}, L_{p}$ and $L_{r}$.

$$
\begin{gathered}
L_{b}=44 \mathrm{in} \\
L_{p}=1.76 r_{y} \sqrt{\frac{E}{F_{y}}}=1.76(3.76) \sqrt{\frac{29000}{50}}=159 \mathrm{in} \\
L_{r}=672 \mathrm{in}
\end{gathered}
$$

4. Since $L_{b}<L_{p}$, LC-SA could fail by reaching the plastic moment. Thus,

$$
M_{C L}=M_{p}=Z_{x} F_{y}=(234)(50)=11,700 \text { kips.in }
$$




\subsubsection{Acceptance Criteria Check}

For $0.2<\frac{P_{U F}}{P_{U L}}<0.5$, combined strength is considered to be deformation controlled. In order to choose the proper $m$ factor, the following conditions must be satisfied:

$$
\begin{aligned}
& \frac{b_{f}}{2_{t f}} \leq \frac{52}{\sqrt{F_{y}}}=7.15 \leq \frac{52}{\sqrt{50}}=7.35 \Rightarrow \checkmark \\
& \frac{h}{t_{w}} \leq \frac{260}{\sqrt{F_{y}}}=17.7 \leq \frac{418}{\sqrt{50}}=36.77 \Rightarrow \checkmark
\end{aligned}
$$

Therefore, the $m$ factor for the CP performance level is given by Equation

$$
\begin{gathered}
m=12\left(1-\frac{5}{3} \frac{P}{P_{C L}}\right) \\
m=12\left[1-\frac{5}{3}(0.31)\right]=5.7
\end{gathered}
$$

The acceptance criteria for LC-SA regarding combined strength shall be evaluated as follows, which satisfies the $\mathrm{CP}$ acceptance criteria.

$$
\begin{gathered}
\frac{P_{U F}}{P_{C L}}+\frac{8}{9}\left[\frac{M_{x}}{\kappa m M_{C E}}\right] \leq 1.0 \\
\frac{602}{1,921}+\frac{8}{9}\left[\frac{6,119}{(1)(5.7) 11,700}\right]=0.39<1.0 \Rightarrow \checkmark
\end{gathered}
$$

Tables (9.4) and (9.5) indicate the DCR values for columns shown in Figure (9.6), subjected to compression and tension forces, respectively. 
Table 9.4: DCR values for columns subjected to compression forces on the LCF 2story building.

\begin{tabular}{ccccccc}
\hline LC-2 & $P_{U F}$ & $P_{C L}$ & $P_{U F} / P_{C L}$ & $\mathrm{~m}$ & $M_{U F}$ & $\mathrm{DCR}$ \\
\hline C5 & 104 & 1910 & 0.05 & 8 & 4156 & 0.07 \\
$\mathrm{C} 4$ & 231 & 1910 & 0.12 & 8 & 4768 & 0.11 \\
$\mathrm{C} 3$ & 408 & 1921 & 0.21 & 8 & 5444 & 0.27 \\
$\mathrm{C} 2$ & 602 & 1921 & 0.31 & 5.7 & 6119 & 0.39 \\
$\mathrm{C} 1$ & 791 & 1935 & 0.41 & 3.8 & 5312 & 0.51 \\
$\mathrm{GC} 2$ & 25 & 1822 & 0.01 & 8 & 2353 & 0.03 \\
$\mathrm{GC} 1$ & 54 & 1822 & 0.03 & 8 & 2113 & 0.04 \\
\hline
\end{tabular}

Table 9.5: DCR values for columns subjected to tension forces on the LCF 2-story building.

\begin{tabular}{ccccc}
\hline $\mathrm{LC}-1$ & $\mathrm{~T}$ & $\mathrm{mt}$ & $M_{U F}$ & $\mathrm{DCR}$ \\
\hline $\mathrm{T} 5$ & 199.0 & 5 & 4158 & 0.07 \\
$\mathrm{~T} 4$ & 496.0 & 5 & 4921 & 0.14 \\
$\mathrm{~T} 3$ & 856.0 & 5 & 5540 & 0.18 \\
$\mathrm{~T} 2$ & 1314 & 5 & 6119 & 0.24 \\
$\mathrm{~T} 1$ & 1857 & 5 & 5309 & 0.28 \\
\hline
\end{tabular}

\subsection{Observations}

Even though ASCE/SEI 41 (2013) is a performance-based engineering standard, some of the factors used toward seismic assessment are irrational factors, and caution must be exercised. To put things in perspective, modern codes have a response modification factor, $R$, which reduces the demands, whereas ASCE/SEI 41 (2013) have a component modification factor, $m$, which increases the demands. $R$ is related to the system while $m$ is related to component level. 
At the end of the seismic assessment, a building will be categorized into pass or fail in regards to the component level response and this might not be the proper way to seismically assess a building.

A LCF building was evaluated by current performance-based engineering guidelines, and although the acceptance criteria for beams and columns were within the acceptable margin and corresponded to what was observed during the LCF experiments, the results for the replaceable shear links indicated the contrary when compared to the LCF experimental results in terms of failure, suggesting that further investigating is needed. 


\section{Chapter 10}

\section{Design Recommendations}

This chapter provides design recommendations for LCF buildings. These recommendations are based on the analyses conducted for this research combined with the author's practical experience. First, seismic performance factors found for the LCF system is compared to conventional systems, followed by a proposed LCF section for the current seismic provisions and, finally, structural detailing for horizontal diaphragm, replaceable link and foundation, are presented.

\subsection{Seismic Performance Factors}

According to FEMA P-695 (2009) seismic performance factors are used in current building codes and standards to estimate strength and deformation demands on seismic-force-resisting systems that are designed using linear methods of analysis, but are responding in the nonlinear range. Malakoutian (2012) numerically quantified two seismic performance factors for LCF buildings using a recommended methodology (FEMA P-695, 2009) for reliably quantifying building system performance and response parameters for use in seismic design. The 
seismic performance factors numerically quantified were: the response modification factor, $R=8.0$, and the deflection amplification factor, $C_{d}=5.5$. Those values are comparable to SMRFs coefficients.

One of the main objectives of this dissertation was to experimentally determine the overstrength coefficient, $\Omega_{0}$, which is also a seismic performance factor. Chapter (6) has shown that, for the LCF-5L specimen tested, $\Omega_{0}$ was equal to 2.0, which is lower than those for SMRFs and EBFs, which are $\Omega_{0}=3.0$ and $\Omega_{0}=2.5$, respectively. The overstrength coefficient might be considered to provide a reasonable estimate of the reserve strength attributable to a rapid return to occupancy level of performance. Table (10.1) indicates the summary of seismic performance factors recommended for LCF buildings.

Table 10.1: Seismic performance factors for LCF buildings.

\begin{tabular}{cccc}
\hline System & $\mathrm{R}$ & $C_{d}$ & $\Omega_{0}$ \\
\hline $\mathrm{LCF}$ & 8.0 & 5.5 & 2.0 \\
\hline
\end{tabular}

\subsection{Proposed LCF Section for the Seismic Provisions}

\section{Scope}

Linked Column Frames (LCF) of structural steel shall be designed in conformance with this section.

\section{Basis of Design}

LCF designed in accordance with these provisions are expected to provide 
significant inelastic deformation capacity through flexural yielding of the beams and shear yielding of links. LCF shall be limited to low and midrise buildings. Linked columns shall be designed to remain elastic under the forces that can be generated by the fully yielded and strain-hardened replaceable link.

\section{Analysis}

The required strength of columns, beams, replaceable links, and connections in LCF shall be based on the load combinations in the applicable building code that include the amplified seismic load. In determining the amplified seismic load, the effect of horizontal forces including overstrength, $E_{m h}$, shall be taken as the forces developed in the member assuming the forces at the ends of the replaceable links correspond to the adjusted replaceable link shear strength. The adjusted replaceable link shear strength shall be taken as $R_{y}$ times the replaceable link nominal shear strength, $V_{n}$, given in Section F.5b(2a) multiplied by 1.8 for I-shaped replaceable links.

\section{System Requirements}

\section{(a) Displacement Ratio}

The following relationship shall be satisfied while designing the LCF system:

$$
\frac{\Delta_{y L C}}{\Delta_{y M F}}=\frac{V_{L C} K_{M F}}{V_{M F} K_{L C}}
$$


where $\Delta_{y L C}$ is the first yield displacement at the replaceable link, $\Delta_{y M F}$ is the first yield displacement at the gravity beam on the moment frame, $V_{L C}$ and $V_{M F}$ are the idealized lateral strengths, and $K_{L C}$ and $K_{M F}$ are the lateral stiffness values of Linked Column (LC) and Moment Frame (MF) subcomponents, respectively.

User Note: Ratios less than unity describe structural systems in which LC reaches plasticity at lower displacements than MF, thereby providing the potential for rapid return to occupancy performance level. The larger the difference between the yield displacements, the larger the drift range in which rapid return to occupancy can occur.

\section{(b) Link Rotation Angle}

The replaceable link rotation angle shall not exceed 0.08 rad for links of length $1.6 M_{p} / V_{p}$ or less, where $M_{p}$ is the nominal plastic flexural strength and $V_{p}$ is the nominal shear strength of an active replaceable link.

\section{Members}

\section{(a) Basic Requirements}

Beam and column members shall satisfy the requirements of Section D1.1 for highly ductile members, unless otherwise qualified by tests. 
Structural steel beams in LCF are permitted to be composite with a reinforced concrete slab to resist gravity loads.

\section{(b) Expected Vertical Axial Strength of Linked Column}

The expected vertical axial strength of the linked column, $P_{n}$, shall be:

$$
P_{n}=\Omega n V_{p}
$$

where $\Omega$ is the overstrength factor and is taken equal 2.0, $n$ is number of replaceable links and $V_{p}$ is the plastic shear yield strength.

\section{(c) Replaceable Links}

Replaceable links designed as short links positioned between the linked columns shall be provided. The replaceable link shall be considered to extend from column face to column face for link-to-column connections. Replaceable links shall be I-shaped cross sections and shall satisfy the requirements of Section D1.1 for highly ductile members. Use of parallel web stiffeners are permitted for the replaceable link components.

User Note: The use of parallel web stiffeners is an effort to provide elastic connections that shift plastic strains away from critical welds, thereby avoiding some of the failures observed in past tests on similarly detailed link-to-column eccentrically 
braced frames.

\section{(d) Protected Zones}

The region at each end of the gravity beam (moment side) subjected to inelastic straining shall be designated as a protected zone, and shall satisfy the requirements of Section D1.3. The extent of the protected zone shall be as designated in ANSI/AISC 358, or as otherwise determined in a connection prequalification in accordance with Section $\mathrm{K} 1$, or as determined in a program of qualification testing in accordance with Section K2.

User Note: The plastic hinging zones ate the ends of LCF beams (moment side) should be treated as protected zones. The plastic hinging zones should be established as part of a prequalification or qualification program for the connection, per Section E3.6c. In general, for unreinforced connections, the protected zone will extend from the face of the column to one half of the beam depth beyond the plastic hinge point.

Replaceable links in LCFs are a protected zone as well, and shall satisfy the requirements of Section D1.3.

\section{Connections}

\section{(a) Demand Critical Welds}


The following welds are demand critical welds, and shall satisfy the requirements of Section A3.4b and I2.3:

(1) Groove welds at column splices.

(2) Complete-joint-penetration groove welds of gravity beam flanges to gravity column, unless otherwise designated by ANSI/AISC 358 , or otherwise determined in a connection prequalification in accordance with Section K1, or as determined in a program of qualification testing in accordance with Section K2.

(3) Partial-joint-penetration groove welds of replaceable links connecting flanges to end-plate connections.

User Note: Weld access hole or rat hole in not permitted on flange welds.

(4) Partial-joint-penetration groove welds of replaceable links connecting web to end-plate connections.

\section{(b) Beam-to-Column Connections (Moment Side)}

Beam-to-column connections (moment side) used in the seismic force resisting system (SFRS) shall be capable of accommodating a story drift angle of at least 0.025 rad. Beam-to-column connections (moment side) and link-to-column connections shall be fully restrained moment connections and shall use extended end-plate connections.

(c) Beam-to-Column Connections (Shear Side) 
Beam-to-column connections (shear side) used in the SFRS shall be capable of accommodating a story drift angle of at least $0.025 \mathrm{rad}$. Beam-to-column connections (shear side) shall be shear connections.

\section{(d) Link-to-Column Connections}

Link-to-column connections shall be fully restrained (FR) moment connections and shall satisfy the following requirements:

(1) The connection shall be capable of sustaining a link rotation angle of $0.08 \mathrm{rad}$.

(2) The shear resistance of the connection, measured at the required link rotation angle, shall be at least equal to the expected shear strength of the link, $R_{y} V_{n}$, as defined in Section F3.5b(2).

Link-to-column connections shall be extended end-plate moment connections with parallel web stiffeners.

(e) Panel Zone

Panel zone criteria shall comply with the requirements of Section E3.6e.

(f) Continuity Plates

Continuity plates shall be provided in accordance with the provisions of Section E3.6f.

\section{(g) Column splices}

Column splices shall be provided in accordance with the provisions 258 
of Sections D2.5 and E3.6g.

\subsection{Horizontal Diaphragm Load Transfer}

Roof and floor framing systems have the primary function of supporting the gravity loads and to transfer these loads to the lateral system. In this regard, roof and floor framing systems, used primarily to resist gravity loads, are also designed as horizontal diaphragms. For analysis purposes diaphragms are classified as rigid, flexible and semi-rigid based upon the relative rigidity between the horizontal diaphragm and the lateral resisting element.

In general, diaphragms made of metal deck with concrete fill is considered to be rigid. The common standard width for metal deck is usually $36 \mathrm{in}$. center to center of the end flutes and the common practice is for the deck to be fastened to the steel framing member using $5 / 8 \mathrm{in}$. diameter puddle welds in the flutes or simply utilize steel headed-stud anchors.

For a LCF building, the load path goes from the gravity beam to the linked column, which in turn goes to the foundation element. The connection between the gravity beam and the linked column was considered to be a shear connection subjected to combined shear and axial load. In order to transmit these loads to the linked column, it is required to design drag struts or collectors as recommended in Figure (10.1). The idea is to decouple shear and axial forces, the shear force is transmitted to the steel stem through the double angles, whereas 
the axial force is transmitted via threaded rods on each side of the GB connecting the gravity beam itself to the steel stem, which in turn is connected to the linked column.

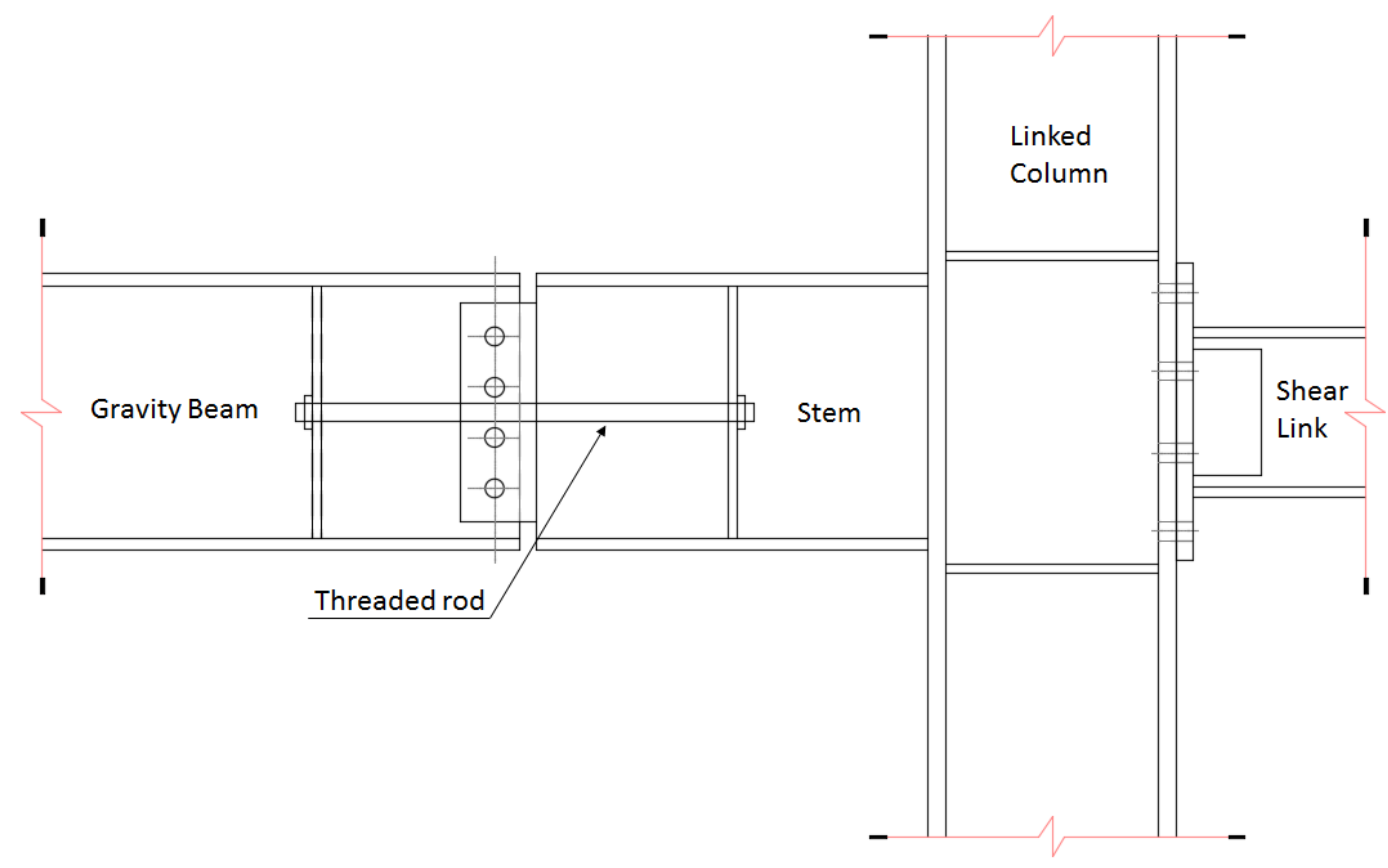

Figure 10.1: Horizontal diaphragm load transfer.

\subsection{Replaceable Link}

The behavior of shear dominated links on EBFs have been comprehensively studied considering link and beam sections as a unit. However, very limited research on the design of EBFs with replaceable links has been done to date. This dissertation was the first time that several replaceable links were tested all together within the LCF system. The intent of using this concept was to isolate the formation of plastic shear hinge through practical connection details 
(Lewis, 2010) for the replaceable link components in an effort to provide elastic connections that shift plastic strains away from critical welds, thereby avoiding some of the failures observed in past tests on similarly detailed link-to-column EBFs. Unlike EBFs, the replaceable shear links are bolted to the columns to facilitate post-earthquake replacement. Even when residual drift results in the structure, the permanent deformations are locked in the replaceable shear links, which can be removed using a plasma cutter or an oxygen-acetylene torch. Figure (10.2) shows the recommended detailing for the replaceable shear link, where the parallel web stiffener (on both sides) has the same thickness of the web and is fillet welded to the link web, partial-joint-penetration groove welding is used to connect flanges to end-plate connections and also to connect web to end-plate connections.

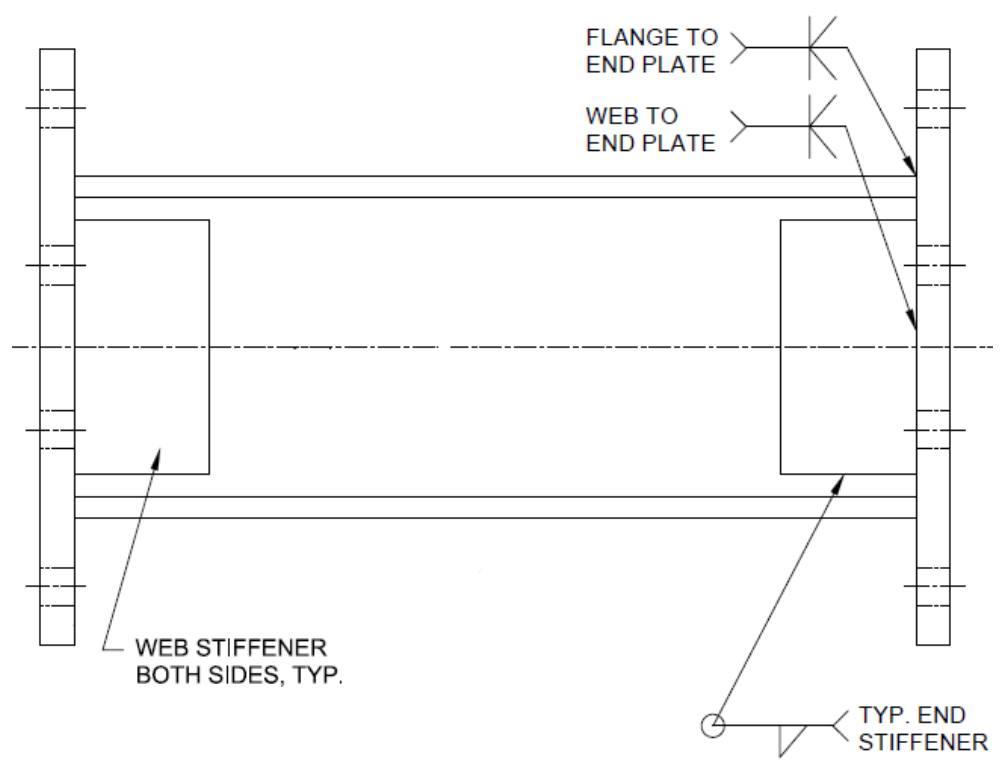

Figure 10.2: Replaceable shear link. 


\subsection{Foundation}

A critical consideration for the satisfactory LCF performance is the proper design of the foundation elements. LCF experimental tests had shown large axial forces on foundation elements and design of such elements needed to be addressed. The selection of a foundation type involves not only structural considerations, but also geotechnical factors. In general, a pile foundation is required when the soil does not have bearing capacity for the structure to withstand or when the imposed loads are very heavy.

This section discusses a suggested pile foundation detailing for LCF buildings. A LCF pile foundation consists of three components: pile cap, group of piles and base plate/anchor rods. Figures (10.3) to (10.5) give a typical general layout for this type of foundation. For practical considerations, a single pile cap is suggested for both linked columns. The pile cap is a reinforced concrete block on the head of a group of piles, to transmit the load from the LCF system to the group of piles and then to the soil. Suggested plane dimensions and corresponding main bar and secondary steel details are shown in Figure (10.3). 

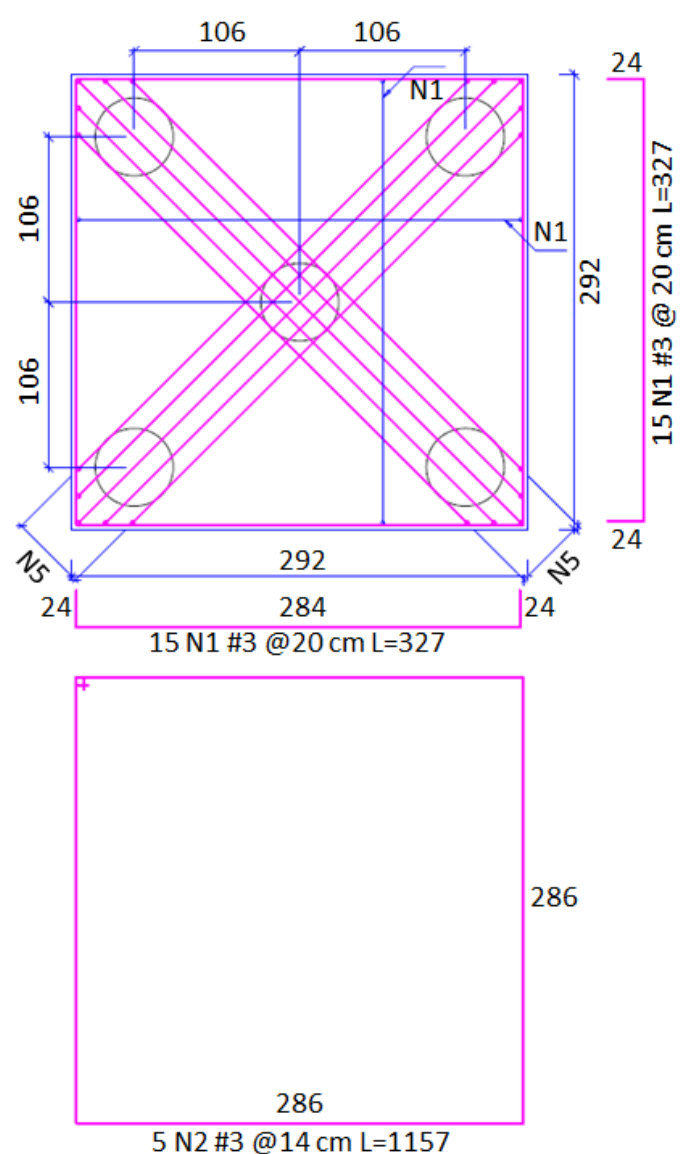

Figure 10.3: Pile cap plan view.

The number of piles depends on the axial load from the linked columns and the piles are arranged in a symmetric way with respect to the load. The linked columns are connected to the pile cap which helps the pile group act as an integral unit. Figure (10.4) shows the lateral view for the pile cap in which the linked columns are welded to a base plate, which is in turn bolted to the pile cap through anchor rods. The anchor rods are not shown for clarity. LCF system is designed in a way that plastic hinges are avoided on the columns. Attaching an extra plate connecting the linked columns may introduce a fixed condition 
on the foundation. This explains why the linked columns are welded directly to the base plate in order to simulate a pinned foundation.

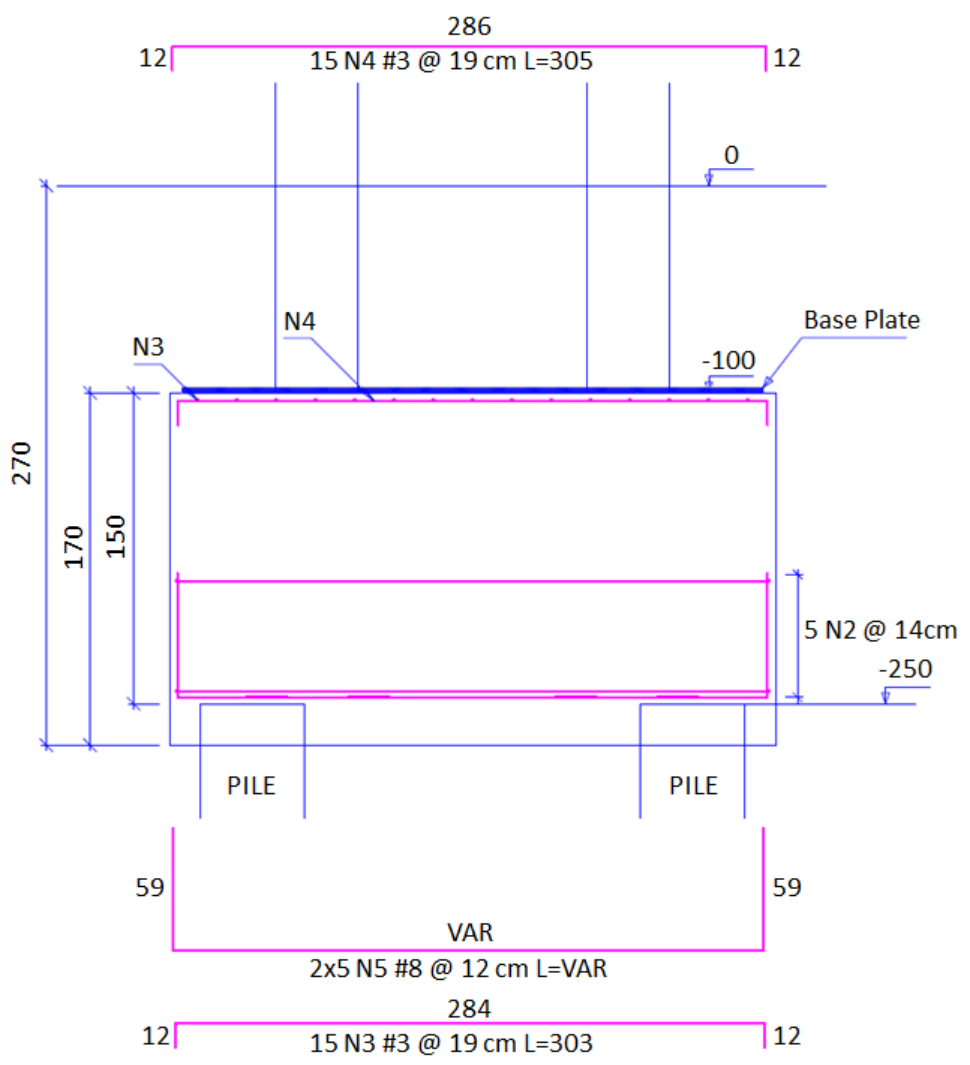

Figure 10.4: Pile cap lateral view without anchor bolts.

The anchor bolts are necessary to connect the base plate to the pile cap in order to avoid any uplift during the seismic event. The anchor bolts contain minimum bolt aspect ratio and minimum bolt/joint stiffness ratio. These requirements generally result in anchor bolts close to 1 in. diameter. In addition to the anchor bolts, it is suggested to include a plate at the end of each anchor bolt to increase the anchorage capacity of the anchor bolts. Also, the anchor bolt should be at least 1 meter long. Figure (10.5) shows the base plate plan view 
for the pile cap indicating anchor bolts and corresponding plates (3" $\times 5$ " $\times 1$ ") at the ends of the anchor bolts, while Figure (10.6) shows the pile cap lateral view with anchor bolts.

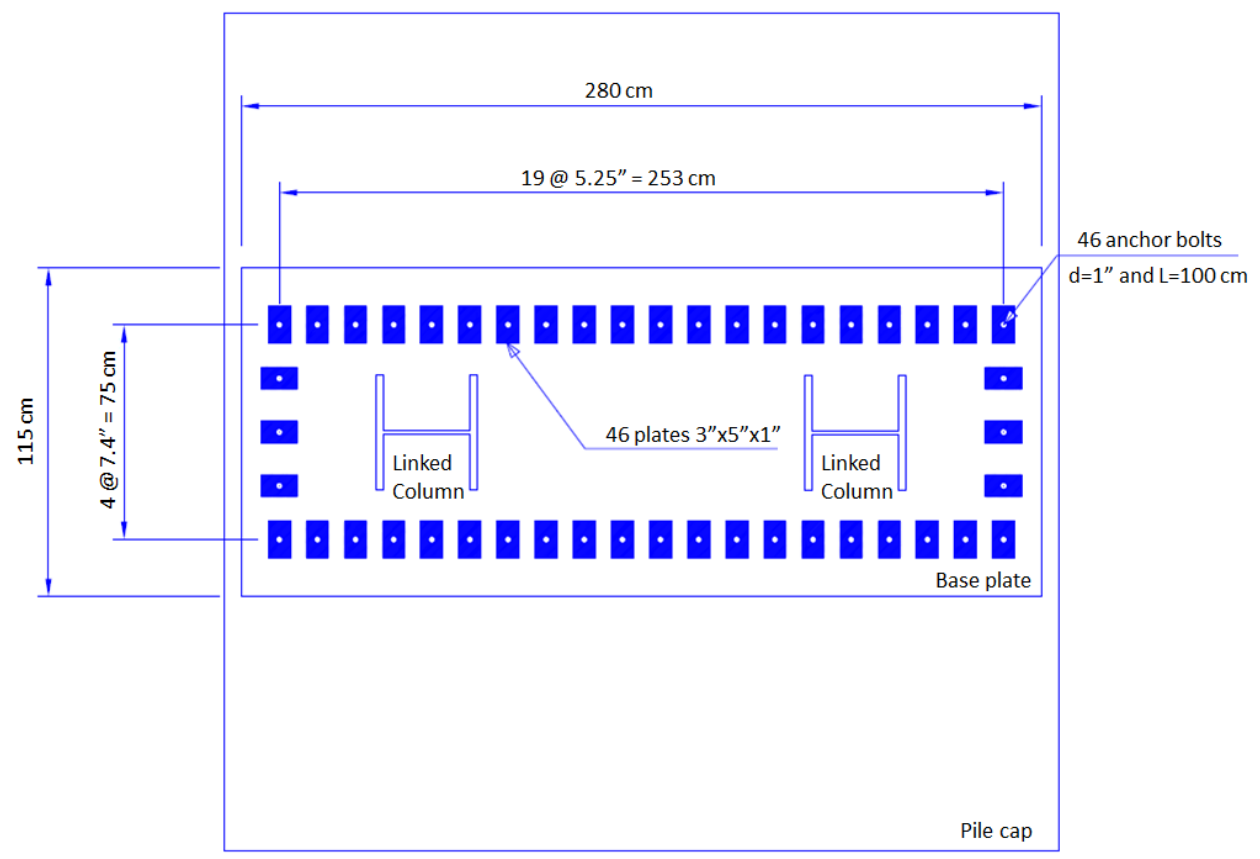

Figure 10.5: Base plate pan view for the pile cap.

Whenever possible, the LCF system should be placed on soils with high bearing capacity to avoid long piles until they reach stronger strata. Additionally, it is recommended to position the base plate of the pile cap at least $1 \mathrm{~m}$ below the ground level and, most likely, the first replaceable shear link will be buried. Figure (10.7) shows the pile cap front view with anchor bolts. Even though the presented detailing is neither a pinned connection nor a fixed connection, all attempts should be made to avoid large moments at the foundation levels. 


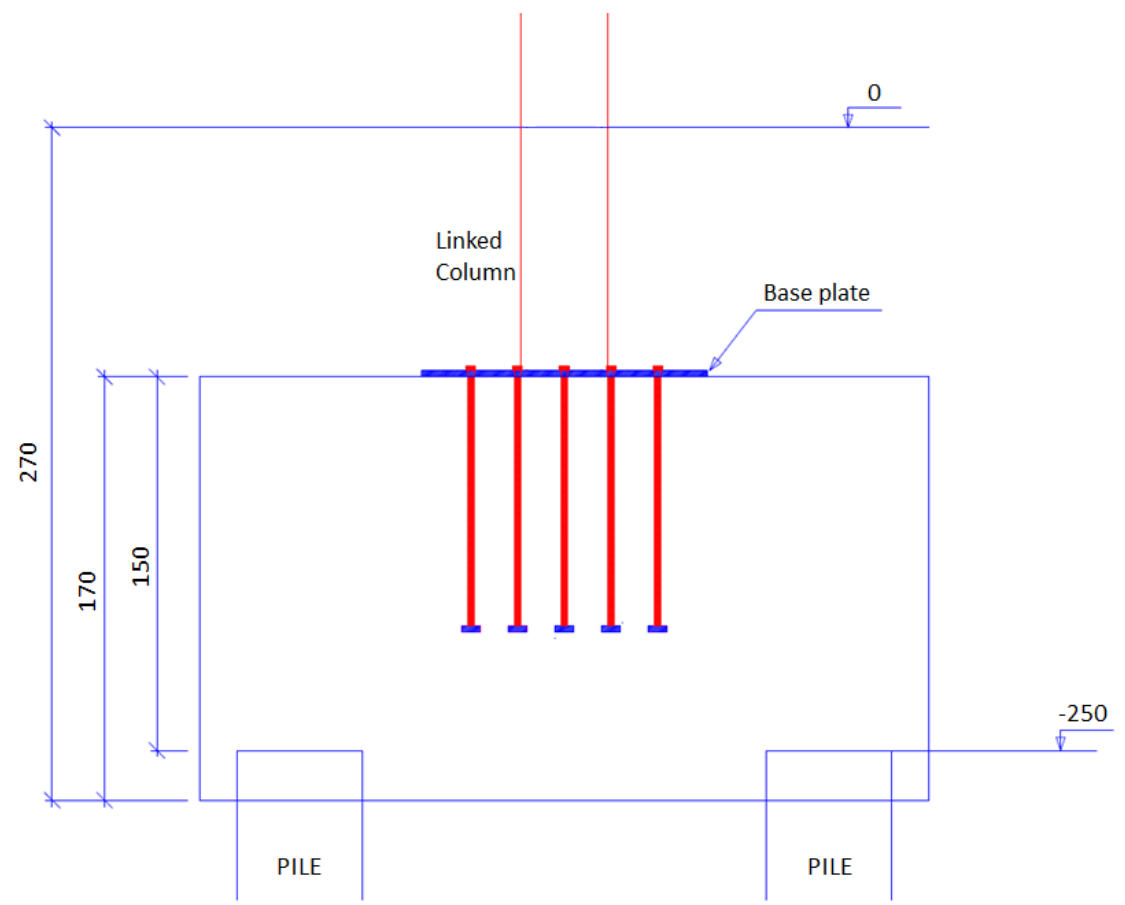

Figure 10.6: Pile cap lateral view with anchor bolts.

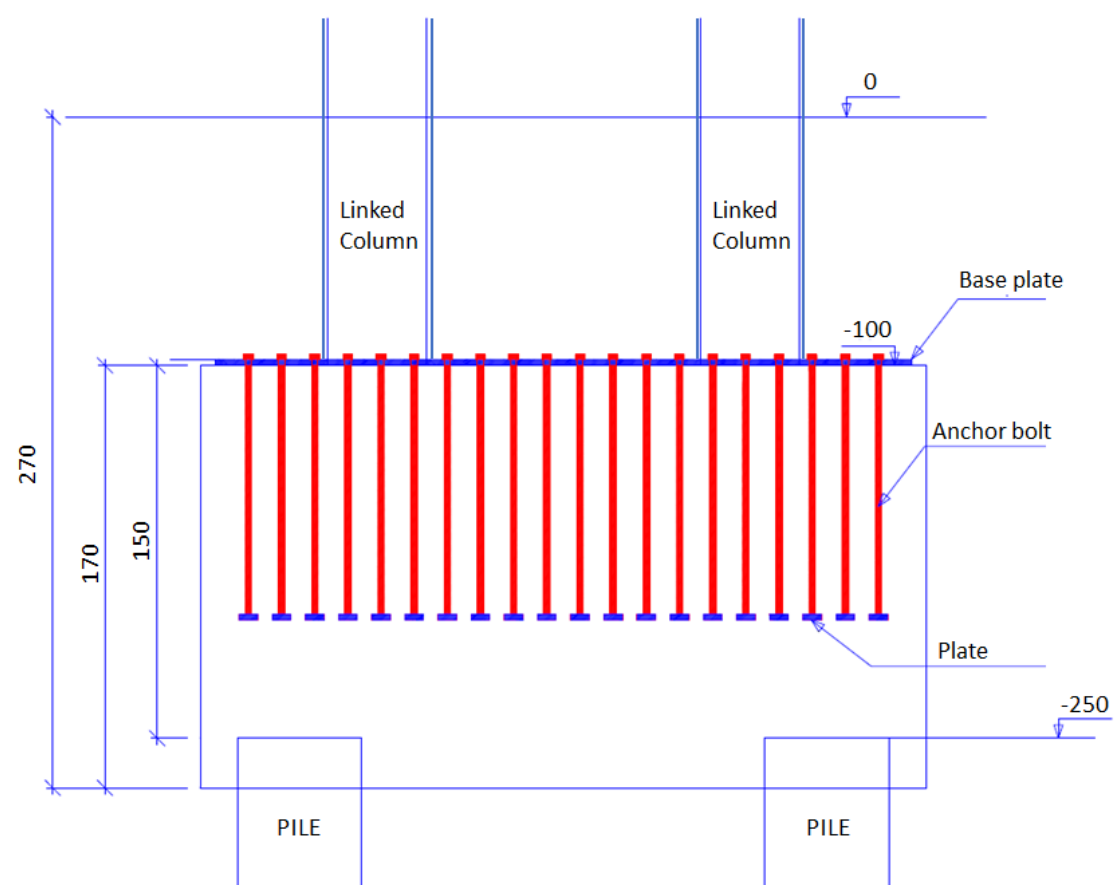

Figure 10.7: Pile cap front view with anchor bolts. 


\section{Chapter 11}

\section{Conclusions and Future Studies}

\subsection{Conclusions}

This study illustrated that a viable non-braced frame alternative is possible with the LCF as a lateral system with potential performance advantages over the more conventional SMRFs. Numerous designs of LCF buildings were completed, numerical system level analyses conducted to evaluate their performance, full-scale experimental tests were performed, and analytical equations were developed. For the cases considered, the following conclusions can be drawn:

- The LCF system takes an innovative step forward by separating the shear link from the framing system, acting as a seismic dissipater. In addition, the link can now be easily replaced and with less disruption after extreme events.

- LCF systems have the potential for effective repair in the rapid return to occupancy performance level.

- Drift criteria controlled the LCF designs based on equivalent static lateral 267 
loads for a moderate to high seismic areas.

- Inter-story drift of the LCF is most effectively controlled by the LCs in the first story and MF beams in the higher stories. However, a design strategy of controlling drifts with LC resulted in lighter structural frame systems than SMRF.

- The LCFs in which the design utilized the LCs to control drift exhibited rapid return to occupancy performance level over a drift range of at least twice the first yield. This performance advantage can be lost if MF are used to control drift during the design process.

- Should drift criteria not be met, increase the stiffness of the links and linked columns first before increasing moment frame beams.

- Unlike SMRFs, LCF systems have the potential for effective repair in the rapid return to occupancy performance level because no gravity members would be damaged. Unlike EBFs, the shear links are bolted to the columns to facilitate post-earthquake replacement. Even when residual drift results in the structure, the permanent deformations are locked in the shear links, which can be removed using a plasma cutter or an oxygen-acetylene torch. And, the elasticity of the MF component of the LCF system would provide restoring forces upon removal of the permanently deformed links.

- The LCF system's ultimate base shear capacity can be significantly lower 
than SMRF designs, resulting in lower demands and improved economy of the foundation. The lower base shears for the LCFs translates to lower demand on the foundation, which results in potential cost savings for the building.

- The LCF strength could be refined by using links as built-up sections/wide flanges such that flexural stiffness of the links is minimally affected while decreasing the strength to reduce foundation forces.

- Using built-up shear links allows for greater design choice to suit the system response over wide-flange sections.

- Parametric studies successfully identified the controlling frame members of the LCF structural system thereby affording the designer precise control over the system as a whole.

- The structural layout was suitable for entire frame validation using hybrid simulation.

- The LCF moment frame remained elastic until rapid return to occupancy performance level was achieved, while links yielded and deformed plastically.

- Shear links shown to be effective in protecting gravity system and participating well past $4 \%$ drift. 
- Limited demands on gravity beams could indicate less rigorous detailing connections. LCF systems tested had a maximum interstory drift of 0.025, suggesting that connection details could be the same as intermediate frames.

- From experimental results, replaceable links can be achieved using web end stiffeners and extended end plate connections.

- LCF specimens exhibited three regions within the lateral response; elastic, yielding of links and yielding of links as well as MF beams. Provided the links are replaceable, these correspond to three distinct performance levels; elastic, rapid return to occupancy and collapse prevention.

- Replaceable links yielded prior gravity beams, all columns remained elastic, and the ultimate system failure mode was ductile and non-catastrophic. The LCF system is a viable system under cyclic and seismic loading, offering a ductile structural system with the ability to rapidly return to occupancy.

- The linked column frame effectively protects the gravity beams as well as the columns such that the structure could rapidly return to occupancy through link replacement.

- The system performance depends in large part on the ability of the link to deform without premature failure. 
- Results have indicated that the LCF systems capability to achieve specific target performance levels and demonstrated the components potential ability to concentrate the desired plasticity within the link.

- Even though an overstrength factor of 2.0 was used for the LCF design, a difference of about $27 \%$ was noted between the LCF design value and the maximum axial load on the linked columns, suggesting that a overstrength factor greater than 2.0 might be considered in the future, while considering the collapse prevention performance level.

- LCF system allowed the replacement of the shear links with minimal disruption.

- A design methodology was developed for low-to-medium height buildings.

- A simple formulation was presented for the development of closed-form expressions of the lateral stiffness of the linked column steel frame system and also to size gravity beam members. In regards to the lateral stiffness, two approaches were presented based on classical methods of structural analysis.

- The analytical examples demonstrated that the prediction of the member sizes is reasonable and illustrated the applicability and accuracy of the proposed formulations. By the same token, expressions developed for a quick estimation of the axial reaction on the foundation element was also 
presented.

- A LCF building was evaluated by current performance-based engineering guidelines, and although the acceptance criteria for beams and columns were within the acceptable margin and corresponded to what was observed during the LCF experiments, the results for the replaceable shear links indicated the contrary when compared to the LCF experimental results in terms of failure.

\subsection{Suggestions for Future Studies}

- System level approaches for taller buildings. One could potentially use a belt truss on the top of the building and use of more linked columns along the building in order to change the stiffness of the entire building.

- Change of boundary conditions within the system in order to better transmit the diaphragm loads into the linked columns.

- Potential use of concrete-filled steel tube column for the linked columns.

- Use of replaceable shear link designs in conventional EBF systems or as external energy dissipaters for other self-centering systems that may be based on rocking principals.

- Development of structural optimization models in regards to cost and weight. 
- Include shear deformation on the analytical equations for lateral stiffness.

- Development of economic studies to compare initial cost versus life cycle cost.

- Development of detail connection for foundation due to large axial forces.

- Intermediate or long links are also potentially possible in LCF designs, however the effects on meeting drift criteria and building member sizes had not been investigated in this research.

- Further refinement of the system could be realized through greater integration of experimental and numerical data. 


\section{References}

ANSI/AISC 341, 2010. Seismic Provisions for Structural Steel Buildings. In American Institute of Steel Construction, Inc., Chicago, Illinois, USA. [11, $16,18,19,39,75,78,80,133,169,177,178,191]$

AISC, 2011. Steel Construction Manual. 14th Edition. In American Institute of Steel Construction, Inc., Chicago, Illinois, USA. [39, 74, 81, 82, 85, 86, $87,178,180,182,190,204]$

ASCE, 2003. Seismic Evaluation of Existing Buildings. In ASCE/SEI 31-03. American Society of Civil Engineers, Reston-VA, USA. [217]

ASCE, 2006. Seismic Rehabilitation of Existing Buildings. In ASCE/SEI 41-06. American Society of Civil Engineers, Reston-VA, USA. [217]

ASCE, 2010. Minimum Design Loads for Buildings and other Structures. In ASCE/SEI \%-10. American Society of Civil Engineers, Reston-VA, USA. $[18,29,39,40,42,51,57,61,128,187,192,219]$

ASCE, 2013. Seismic Evaluation and Retrofit of Existing Buildings. In 
ASCE/SEI 41-13. American Society of Civil Engineers, Reston-VA, USA. $[217,218,219,241,243,249]$

Astanesh, A. \& Zhao, Q., 2002. Cyclic Behavior of Steel Shear Wall Systems. In Proceedings of the Annual Stability Conference, Structural Stability Research Council, Seattle, Washington. [19]

ATC-24, 1992. Guidelines for Cyclic Seismic Testing of Components of Steel Structures. In Applied Technology Council 1992; Report No. ATC-24., Redwood City, California, USA. [-]

AWS, 2004. Structural Welding Code - Steel. In American Welding Society, Miami, Florida, USA. [88]

Bernal, D. (1987). Amplification Factors for Inelastic Dynamic P-delta Effects in Earthquake Analysis. In Earthquake Engineering 83 Structural Dynamics, International Association for Earthquake Engineering, volume 15 (5), pp. 635-651. [53]

Bruneau, M., Uang, C. M \& Whittaker, A., 1998. Ductile Design of Steel Structures. In McGraw-Hill, Boston, Massachusetts, USA. [8, 77]

Chao, S., Khandelwal, K., \& El-Tawil, S. (2006). Ductile Web Fracture Initiation in Steel Shear Links. In Journal of Structural Engineering, American Society of Civil Engineers, volume 132 (8), pp. 1192-1200. [11] 
Chao, S. \& Goel, S., 2008. Performance-Based Plastic Design of Special Truss Moment Frames. In Engineering Journal, pp. 127-150. [17]

Carter, C. J. 1999. Steel Design Guide No. 13: Stiffening of Wide-Flange Columns at Moment Connections: Wind and Seismic Applications. In American Institute of Steel Construction, Inc., Chicago, Illinois, USA. [-]

Clark, P., Frank, K., Krawinkler, H., \& Shaw, R. (1997). Protocol for Fabrication, Inspection, Testing, and Documentation of Beam-Column Connection Tests and Other Experimental Specimens. In SAC Steel Project Background Document; Report No. SAC/BD-97/02., Whashington, DC, USA. $[-]$

Dimakogianni, D., Dougka, G., Vayas, I., \& Karydakis, P. (2013). Seismic Behavior of Innovative Energy Dissipation Systems FUSEIS 1-2. In Proceedings of the 4 th ECCOMAS Thematic on Computational Methods in Structural Dynamics and Earthquake Engineering, Kos Island, Greece. [21]

Dusicka, P., Itani, A. \& Buckle, I. $\left(2004^{b}\right)$. Evaluation of Conventional and Specialty Steels in Shear Link Hysteretic Energy Dissipators. In Proceedings of the 13th World Conference on Earthquake Engineering, Vancouver, BC, Canada. [133]

Dusicka, P., Itani, A. \& Buckle, I. (2006). Built-up Shear Links as Energy 
Dissipators for Seismic Protection of Bridges. In Proceedings of the International STESSA Conference Behaviour of Steel Structures in Seismic Areas, Philadelphia, Pennsylavania, USA. [15]

Dusicka, P. \& Iwai, R., 2007. Development of Linked Column Frame System for Seismic Lateral Loads. In Proceedings of the American Society of Civil Engineers Structures Congress, Long Beach, California, USA. [25]

Dusicka, P. \& Lewis, G., 2010. Investigation of Replaceable Sacrificial Steel Links. In Proceedings of the 9th U.S. 83 10th Canadian Conference on Earthquake Engineering, Earthquake Engineering Research Institute, Toronto, Canada. $[27,53]$

FEMA 302, 1997. NEHRP Recommended Provisions for Seismic Regulations for New Buildings and Other Structures. In Federal Emergency Management Agency, Washington DC, USA. [54]

FEMA 350, 2000. Recommended Seismic Design Criteria for New Steel Moment-Frame Buildings. In Federal Emergency Management Agency, Washington DC, USA. [81]

FEMA 355-C, 2000. State of the Art Report on Systems Performance of Steel Moment Frames Subjected to Earthquake Ground Shaking. In Federal Emergency Management Agency, Washington DC, USA. [27, 200]

FEMA 356, 2000. Pre-standard and Commentary for Seismic Rehabilitation 277 
of Buildings. In Federal Emergency Management Agency, Washington DC, USA. $[1,34,45]$

FEMA 440, 2005. Improvement of Nonlinear Static Seismic Analysis Procedures. In Federal Emergency Management Agency, Washington DC, USA. $[1,40,193]$

FEMA 445, 2006. Next-Generation Performance-Based Seismic Design Guidelines: Program Plan for New and Existing Buildings. In Federal Emergency Management Agency, Washington DC, USA. [1]

FEMA 461, 2007. Interim Testing Protocols for Determining the Seismic Performance Characteristics of Structural and Nonstructural Components. In Federal Emergency Management Agency, Washington DC, USA. [-]

FEMA p-695, 2009. Quantification of Building Seismic Performance Factors. In Federal Emergency Management Agency, Washington DC, USA. [62, 251]

Filiatrault, A., Restrepo J., \& Christopoulos, C. (2003). Structural SelfCentering Earthquake-Resisting Systems. In Proceedings of the SEAOC Convention, Squaw Creek, California, USA. [20]

Galambos, T., 1998. Guide to Stability Design Criteria for Metal Structures. In Fifth Edition, John Wiley 63 Sons, New York NY, USA. [73]

Ghobarah, A., 2001. Performance-Based Design in Earthquake Engineering: 
State of Development. In Engineering Structures, volume 23, pp. 878-884. $[1]$

Gupta, A., \& Krawinkler, H. (1999). Seismic Demands for Performance Evaluation of Steel Moment Resisting Frames. In Report No. 132, The John A. Blume Earthquake Engineering Center, Stanford University, Stanford, California, USA. [53]

Gupta, A., \& Krawinkler, H. (2000). Dynamic P-Delta Effects for Flexible Inelastic Steel Structures. In Journal of Structural Engineering, American Society of Civil Engineers, volume 126 (1), pp. 145-154. [53]

Hines, E. M., 2009. Eccentric Braced Frame Design for Moderate Seismic Regions. In Structures 2009: Don't Mess with Structural Engineers, pp. 1-10.

IBC, 2012. International Building Code. 2nd Printing. In International Code Council, Inc., Country Club Hills, Illinois, USA. [39, 192]

Kasai, K. \& Popov, E. P. (1986). General Behavior of WF Steel Shear Link Beams. In Journal of Structural Engineering, American Society of Civil Engineers, volume 112 (2), pp. 362-382. [77]

Khoo, H.H., Clifton G.C., Butterworth, J.W., Mathieson, C.D. \& MacRae, 
G.A. (2011). Development of the Self-Centering Sliding Hinge Joint. In Proceedings of the 9th Pacific Conference on Earthquake Engineering, Auckland, New Zealand. [1, 21]

Krawinkler, H., Gupta A., Medina, R., \& Luco, N. (2000). Development of Loading Histories for Testing of Steel Beam-to-Column Assemblies. In SAC Background Report SAC/BD-00/10. [-]

Lai, J-W (2012). Experimental and Analytical Studies on the Seismic Behavior of Conventional and Hybrid Braced Frames. In PhD Dissertation, University of California, Berkeley, California, USA. [88]

Lewis, G. (2010). Replaceable Shear and Flexural Links for the Linked Column Frame System. In MS Thesis, Portland State University, Portland, Oregon, USA. $[2,261]$

Lopes, A. P., Dusicka, P. \& Berman, J. W. (2010). Design of Linked Column Frame System for Dual Shake Table Experiments. In Poster session of the Quake Summit NEES Annual Meeting, San Francisco, California, USA. [69]

Lopes, A. P., Khan, P. \& Dusicka, P. (2011). Drift Control Procedures for the Linked Column Frame System. In Proceedings of the $8^{\text {th }}$ Annual Research Conference at Portland State University, Portland, Oregon, USA. [43]

Lopes, A. P., Dusicka, P. \& Berman, J. W. $\left(2012^{a}\right)$. Design of the Linked Column Frame Structural System. In Proceedings of the International STESSA 
Conference Behaviour of Steel Structures in Seismic Areas, Santiago, Chile. $[2,27,39,42,44,164]$

Lopes, A. P., Dusicka, P. \& Berman, J. W. $\left(2012^{b}\right)$. Linked Column Framing System Analyses Toward Experimental Validation. In Proceedings of the American Society of Civil Engineers Structures Congress, Chicago, Illiinois, USA. [56]

Lopes, A. P., Dusicka, P. \& Berman, J. W. $\left(2012^{c}\right)$. Validation Experiments for the Linked Column Frame System. In Poster session of the Quake Summit NEES Annual Meeting, Boston, Massachusetts, USA. [69]

Lopes, A. P., Dusicka, P. \& Berman, J. W. (2013). Toward Hybrid Simulation of the Linked Column Frame System. In Poster session of the Earthquake Engineering Research Institute Annual Meeting, Seattle, Washington, USA. $[-]$

Lopes, A. P., Dusicka, P. \& Berman, J. W. (2014). Linked Column Frame Steel System Performance Validation using Hybrid Simulation. In Proceedings of the 10th U.S. National Conference on Earthquake Engineering, Earthquake Engineering Research Institute, Anchorage, Alaska. [177]

Lopes, A. P., Dusicka, P. \& Berman, J. W. (2015). Lateral Stiffness Approximation of the Linked Column Steel Frame System. In Proceedings of the 
American Society of Civil Engineers Structures Congress, Portland, Oregon, USA. [-]

Malakoutian, M. (2012). Seismic Response Evaluation of the Linked Column Frame. In PhD Dissertation, University of Washington, Seattle, Washington, USA. $[2,27,29,34,52,56,65,251]$

Malakoutian, M., Berman, J. W. \& Dusicka, P. (2013). Seismic Response Evaluation of the Linked Column Frame. In Earthquake Engineering \& Structural Dynamics, volume 10, pp. 795-814. [164, 180, 181, 182, 183]

Mansour, N. (2010). Development of the Design of Eccentrically Braced Frames with Replaceable Shear Links. In PhD Dissertation, University of Toronto, Toronto, Ontario, Canada. [11]

McCormick, D., Aburano, H., Ikenaga, M., \& Nakashima, M. (2008). Permissible Residual Deformation Level for Building Structures Considering both Safety and Human Elements. In Proceedings of the 14th World Conference on Earthquake Engineering, Beijing, China. [145]

McDaniel, C. C., Uang, C., \& Seible, F. (2003). Cyclic Testing of Builtup Steel Shear Links for the New Bay Bridge. In Journal of Structural Engineering, American Society of Civil Engineers, volume 129 (6), pp. 801809. [17]

McKenna, F. T. (1997). Object-Oriented Finite Element Programming: 282 
Frameworks for Analysis, Algorithms and Parallel Computing. In PhD Dissertation, University of California, Berkeley, California, USA. [73]

Mosqueda, G., Stojadinovic, B., \& Mahin, S. (2005). Implementation and Accuracy of Continuous Hybrid Simulation with Geographically Distributed Substructures. In Technical Report UCB/EERC-2005/02, Earthquake Engineering Research Center, Berkeley, California, USA. [23]

Murray, T. M. (2004). Steel Design Guide No. 4: Extended End-Plate Moment Connections: Seismic and Wind Applications. In American Institute of Steel Construction, Inc., Chicago, Illinois, USA. [81]

Okazaki, O., Arce, G., Ryu, H. \& Engelhardt, M. (2005). Experimental Study of Local Buckling, Overstrength, and Fracture of Links in Eccentrically Braced Frames. In Journal of Structural Engineering, American Society of Civil Engineers, volume 131 (10), pp. 1526-1535. [16]

Palkopoulou, O., Karydakis, P. \& Vayas, I. (2009). Innovative Bracing System for Seismic Resistant Steel Structures. In Proceedings of the International STESSA Conference Behaviour of Steel Structures in Seismic Areas, Philadelphia, Pennsylavania, USA. [27]

Pollino, M. \& Bruneau, M. (2007). Seismic Retrofit of Bridge Steel Truss Piers using a Controlled Rocking Approach. In Journal of Bridge Engineering, American Society of Civil Engineers, volume 12 (5), pp. 600-610. [21] 
Popov, E. P., \& Engelhardt, M. (1988). Seismic Eccentrically Braced Frames. In Journal of Constructional Steel Research, Elsevier, volume 10, pp. 321354. [16]

Ricles, J. M., Sause, R., Garlock, M. M., \& Zhao, C. (2001). Post Tensioned Seismic-Resistant Connections for Steel Frames. In Journal of Structural Engineering, American Society of Civil Engineers, volume 127 (2), pp. 113121. $[1,20]$

Roeder, C. W., Lehman, D. E., \& Christopulos, A. (2006). Seismic Performance of Special Concentrically Braced Frames with Buckling Restrained Braces. In Proceedings of the 8th U.S. National Conference on Earthquake Engineering, San Francisco, California, Paper No. 1503. [19]

Sayani, P. J., Erduran, E., \& Ryan, K. L. (2011). Comparative Response Assessment of Minimally Compliant Low-Rise Base-Isolated and Conventional Steel Moment-Resisting Frame Buildings. In Journal of Structural Engineering, American Society of Civil Engineers, volume 147 (10), pp. 1118-1131. [53]

SAP2000, 2010. SAP2000 Users Manual v.14. In Computers and Structures Inc., Berkeley, California, USA. [34, 45, 73]

Schellenberg, A. H. (2008). Advanced Implementation of Hybrid Simulation. In PhD Dissertation, University of California, Berkeley, California, USA. [-] 
Shing, P. B., \& Mahin, S.A. (1984). Pseudodynamic Test Method for Seismic Performance Evaluation: Theory and Implementation. In Technical Report UCB/EERC-84/01, Earthquake Engineering Research Center, Berkeley, California, USA. [23]

Somerville, P., Smith, N., Punyamurthula, S. \& Sun, J. (1997). Development of Ground Motion Time History for Phase 2 of the FEMA SAC Steel Project. In $S A C$ Joint Venture, Whashington, DC, USA. [54]

Stephens, M. (2011). Numerical and Experimental Analysis of Composite Sandwich Links for the LCF System. In MS Thesis, Portland State University, Portland, Oregon. [2]

Stratan, A., \& Dubina, D. (2004). Bolted Links for Eccentrically Braced Steel Frames. In Connections in Steel Structures V, Amsterdam. [11]

Sumner, E. A. \& Murray, T. M. (2002). Behavior of Extended End-Plate Moment Connections Subjected to Cyclic Loading. In Journal of Structural Engineering, American Society of Civil Engineers, volume 128 (4), pp. 501508. [81]

Sumner, E. A. (2003). Unified Design of Extented End-Plate Moment Connections Subjected to Cyclic Loading. In PhD Dissertation, Virginia Polytechnic and State University, Blacksburg, Virginia, USA. [-]

Thornton, W. A. (1990). Design of Base Plates for Wide Flange Columns 285 
- A Concatenation of Methods. In Engineering Journal, American Institute of Steel Construction, volume 4, pp. 173-174. [82]

Tremblay, R. \& Tirca, L. (2003). Behavior and Design of Multistory Zipper Concentrically Braced Steel Frames for the Mitigation of Soft Story Response. In Proceedings of the International STESSA Conference Behaviour of Steel Structures in Seismic Areas, Naples, Italy. [10] 
Appendix-A HS input file 
File: Numerical Model for LCF syste

Monday, July 20, 2015 4:01 Py

+ units kips and inches

+ Created: 05/2013

+ Start of model generation

+ create ModelBuilder (with two-dimensions and 3 DoF/node)

model BasicBuilder -ndm 2 -ndf 3

source LibUnits.tel

source Wsection.tcl

+ INPUTS

set Lbeam 289.3 ;

set Lcol 110.0 ;

set mass 10.6826

set mass 20.7385

set xDamp 0.02 ;

+ damping ratio

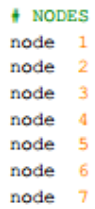

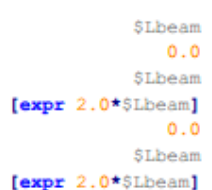

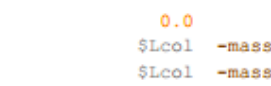

SLcol -mass

[expr $2.0 \star \$ L C O 1]-\operatorname{mas} 3$

$\begin{array}{ll}{[\operatorname{expr} 2.0 * \$ L c o 1]} & - \text { mass } \\ {[\operatorname{expr} 2.0 * \$ L c o 1]} & \text {-mass }\end{array}$

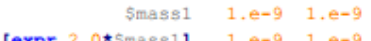

$\begin{array}{rll}\text { Smass1 } & 1 . e-9 & 1 . e-9 \\ \text { Smass2 } & 1 . e-9 & 1 . e-9 \\ \text { [expr 2.0* } \text { Smass2] } & 1 . e-9 & 1 . e-9 \\ \text { Smass2 } & 1 . e-9 & 1 . e-9\end{array}$

4 BOUNDARY CONDITIONS

fix 1 i 10

fix 20010

fix 4010

fix $\begin{array}{lllll}5 & 0 & 1 & 0\end{array}$

fix 7010

+ CONStraints

equalDoF 231

equalDOF 24 is

equalDOF 5 . 1 is

equalDOF 57 ,

+ Columan material, section and element

imaterial

set Fy [expr $50.0 \star 5 \mathrm{ksi}$

set nu 0.3 ;

set Gs [expr \$Es/2./[expr 1+\$nu]]; + Torsional stiffness Modulus

+ strain-hardening ratio

set $R 030$;

t control the transition from elastic to plastic branches

set $C R 1 \quad 0.925$

\# control the transition from elastic to plastic branches 


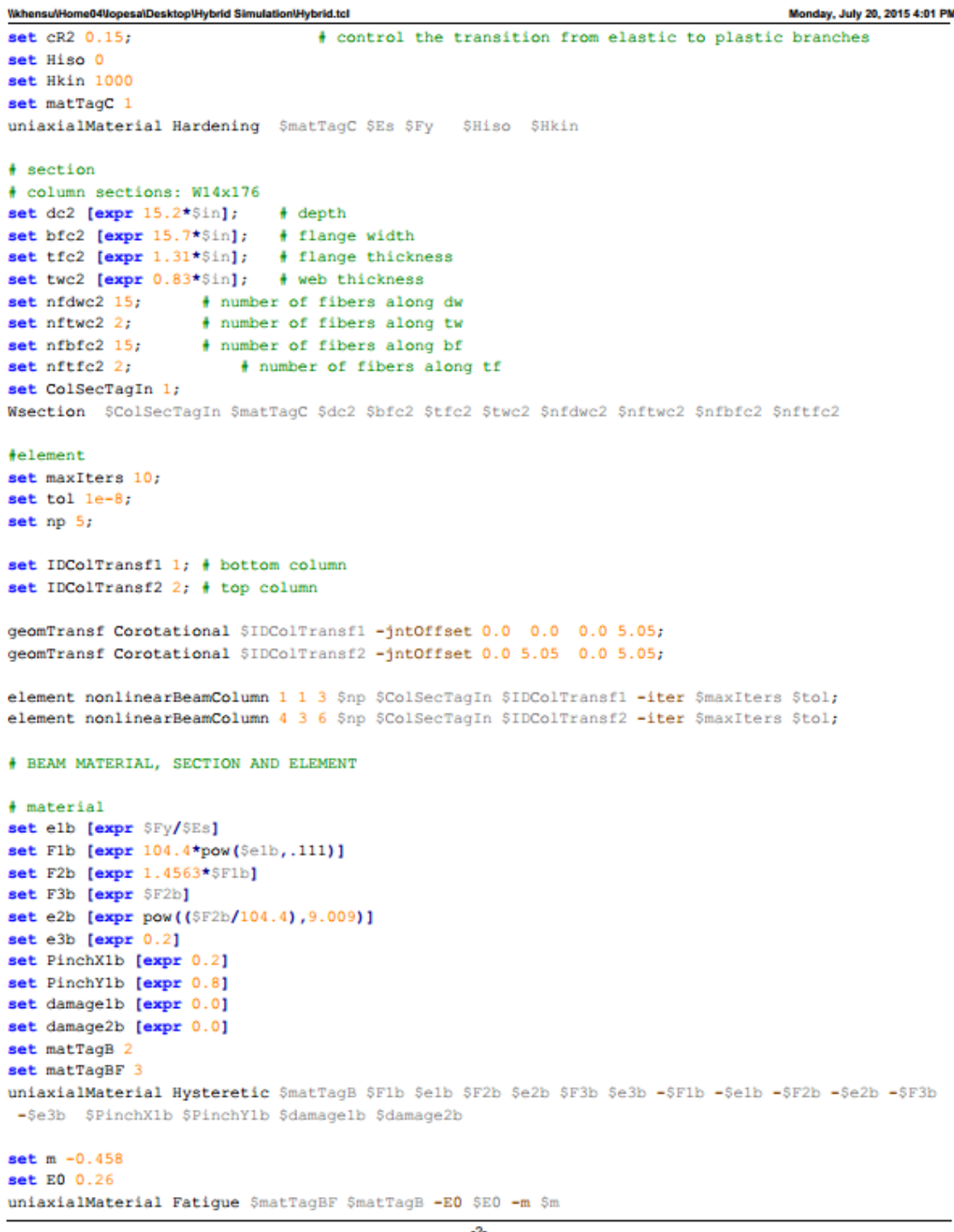


tsection

4 beam sections: w1 $6 \times 57$

set $\mathrm{d} 1$ [expr $16.4 \star \$ 1 \mathrm{n}] ;$; depth

set bf1 [expr 7.12*\$n]; \# flange width

set tf1 [expr 0.715*\$1n]; ; flange thicknes:

set tw1 [expr 0.43*\$in]; \# web thickness

set nfdw1 16; \# number of fibers along di

set nftw1 2; $\quad$ number of fibers along tw

set nfbf1 7; \# number of fibers along bf

set nfte1 2; $\quad$ number of fibers along tf

set BeamSecTag 4

Wsection \$BeamSecTag \$matTagBF \$d1 \$bf1 \$tf1 \$tw1 \$nfdw1 \$nftw1 \$nfbf1 \$nftf1

telement

set IDBeamTransf $13 ;$ i left beam

set IDBeamTransf2 $4 ;$ i right beam

geomTransf Corotational \$IDBeamTransf1 -jntoffset $0.0 \quad 0.07 .6 \quad 0.0$;

geomTransf Corotational \$IDBeamTransf2 -jntoffset $\begin{array}{lllll}7.6 & 0.0 & 0.0 & 0.0 & \text {; }\end{array}$

element nonlinearBeamColumn 223 snp \$BeamSecTag \$IDBeamTransfl -iter \$maxiters $\$$ to

element nonlinearBeamColumn 3 3 4 snp \$BeamSecTag \$IDBeamTransf2 -iter \$maxiters \$tol;

element nonlinearBeamColumn 556 snp \$BeamSecTag \$IDBeamTransf1 -iter \$maxiters \$tol,

element nonlinearBeamColumn $6 \quad 6 \quad 7$ sp $\$$ BeamSecTag $\$$ IDBeamTransf2 -iter $\$$ maxiters $\$$ tol,

+ EXPERIMENTAL SUBSTRUCTURE

loadPackage OpenFresco

expControl SCRAMNet 13810208

expSetup NoTransformation 1 -control 1 -dir 12 -sizeTrialout 22

expsite Localsite 1

Define experimental elements

t left and right columns

4 expElement twoNodeLink SeleTag \$iNode \$jNode -dir \$dirg -site \$siteTag -initstif \$Kij

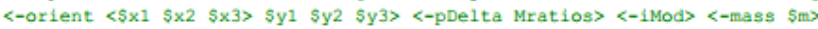

expElement generic 7 -node 25 -dof 1 -dof 1 -site 1 -initstif $1436.0-735.0$-735.0 434.0;

set dt 0.02 ;

set SCALE [expr 0.015]:

set nsteps 8500 ;

set accelseries "Path -filePath se25GM.txt -dt \$dt -factor [expr 1.0*\$SCALE]"

* pattern UniformExcitation Spatterntag \$dir -accel (TimeSeriesType arguements) <-velo \$velo> 


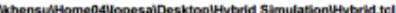

Monday, July 20, 2015 4:01 PW

pattern UniformExcitation 1 1 -accel $\$$ saccelSerles;

+ DAMPING

set MpropSwitch 1.0

set KcurrSwitch 0.0 ;

set Kcommswitch 0.0 ;

set KinitSwitch 1.0 ;

Hintial stifness proportional damping

set nEigenI 1; 4 mode 1

set nEigenJ 2;

set lambdan [eigen [expr $\$ n E i g e n J]$ ]

set lambdaI [1index $\$$ lambdan [expr $\$ n E i g e n I-1]$ ];

set lambdaJ [1index $\$$ lambdan [expr $\$ n E i g e n J-1]$;

t eigenvalue analysis for nEigenJ modes

+ eigenvalue mode 1

set omegaI [expr pow $(51$ lambdaI, 0.5$)$ ],

set omegaJ [expr pow $(\$ 1$ ambdaJ, 0.5$)$ ];

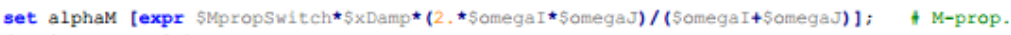

damping; $D$ - alphaM*M

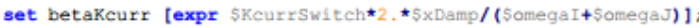

t current-K

tbeatKeurr*KCurrent

+ last-committed $\mathrm{K}$;

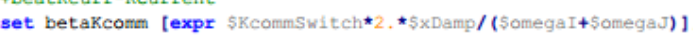

+betaKcomm*KlastCommitt

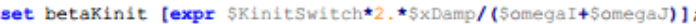

initial-K; +beatKinit*Kini

rayleigh \$alphaM \$betakcurr \$betakinit \$betakcomm;

+ RAYLEIGH damping

+ anALYSis

Create the system of equations

system BandGeneral;

t create the DOF numberer

numberer $\mathrm{RCM}$;

\# create the constraint handler

constraints Transformation;

t create the convergence test

test FixedNumIter 10 ;

4 create the integration scheme

integrator NewmarkHSFixedNumIter $0.5 \quad 0.25$,

t create the solution algorithm

algorithm Newton;

* create the analysis object

analysis Transient;

+ RECORDERS

t experimental nodes 
i calculated recorders

recorder Node -file Node_Dsp.out -time -node 12334567 -dof 1 disp recorder Node -file Node_Rot.out -time -node 122345567 -dof 3 disp

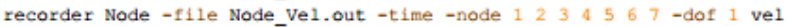

recorder Node -file Node_Acc.out -time -node 12344567 -dof 1 accel

+ control recorders

expRecorder Control -file Control_ctridsp.out -time -control 1 ctr1Disp

expRecorder Control -file Control_daqDsp.out -time -control 1 daqDisp

expRecorder Control -file Control_daqFrc.out -time -control 1 daqForce

recorder Element -file Exp_Elmt_Frc.out -time -ele 7 forces

recorder Element -file Columnforces.out -time -ele 14 forces

recorder Element -file Beamforces.out -time -ele 2356 forces

t open output file for writing

set outFileID [open elapsedTime.txt w]

+ perform the transient analysis

set dtanalysis 0.01 ;

set tTot [time $t$

for \{set $i$ if $\{\$ 1<$ Snsteps\} \{incr i\} \{

set $t$ [time fanalyze 1 [expr \$dtanalysis]l]

puts "step \$1"

puts SoutFileID $\$ t$

b)

puts "\nElapsed Time $=$ \$tTot $\mid \mathrm{n}$ "

+ close the output file

close SoutFlleID

wipe

exit 
Appendix-B Fabrication drawings 


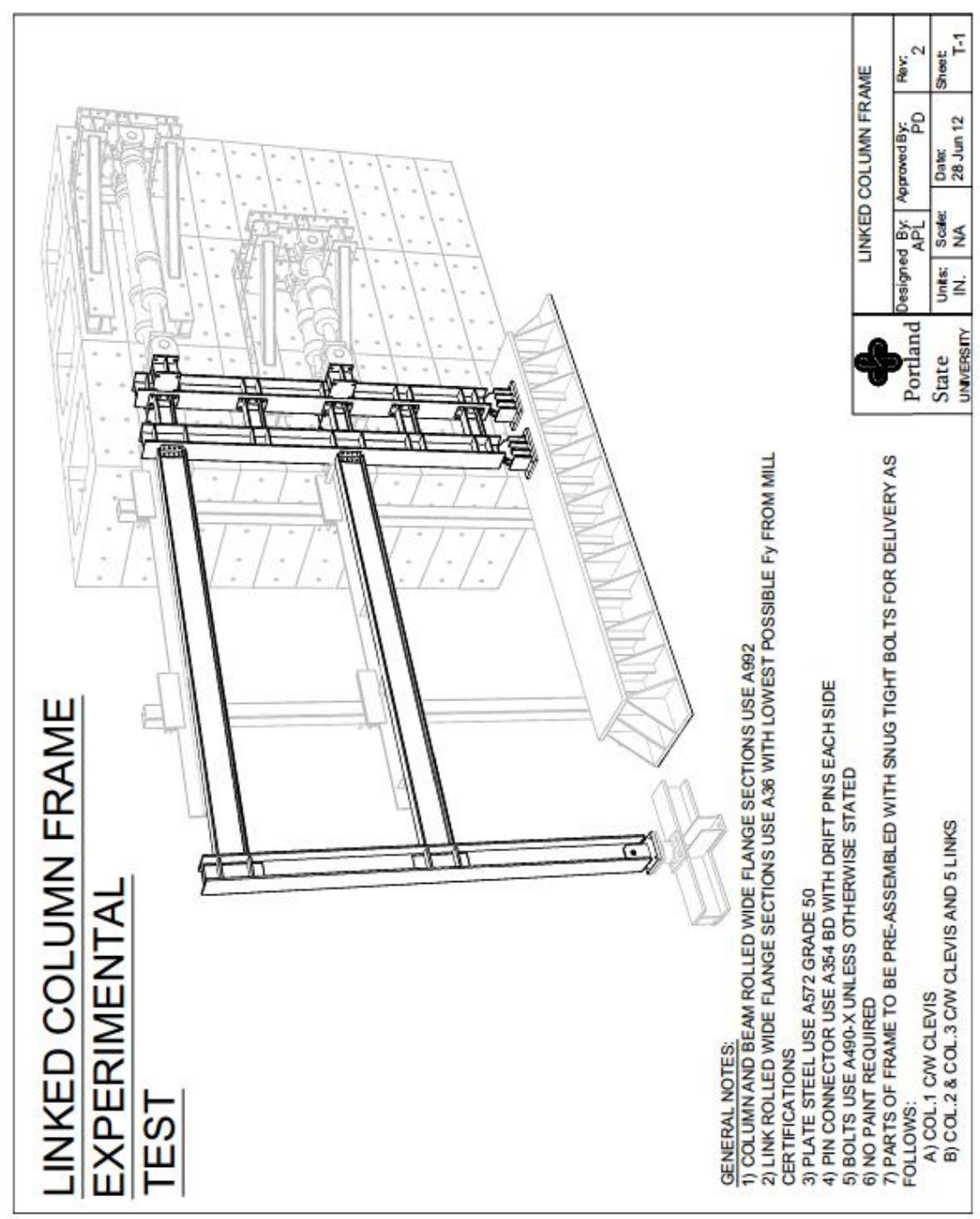




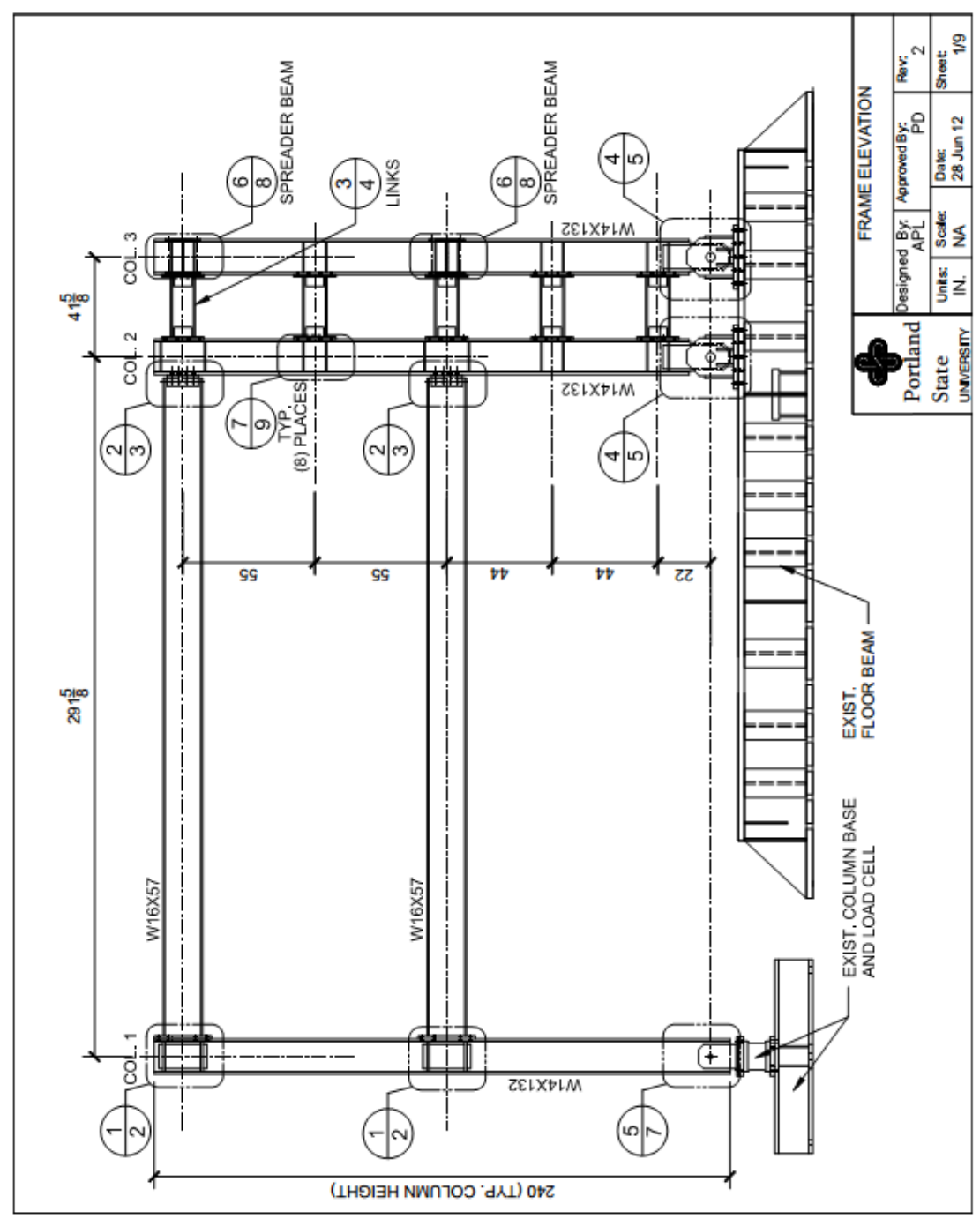




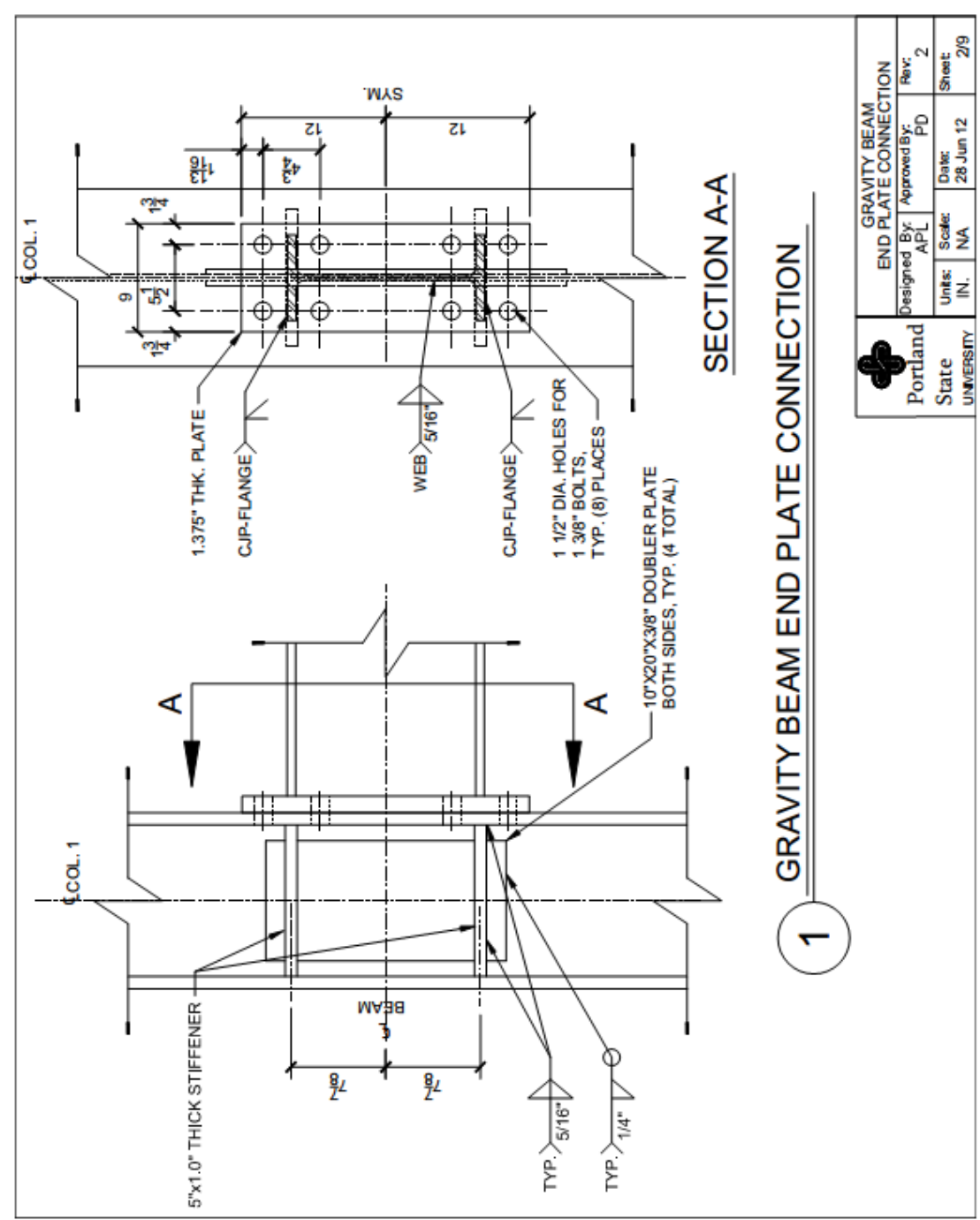




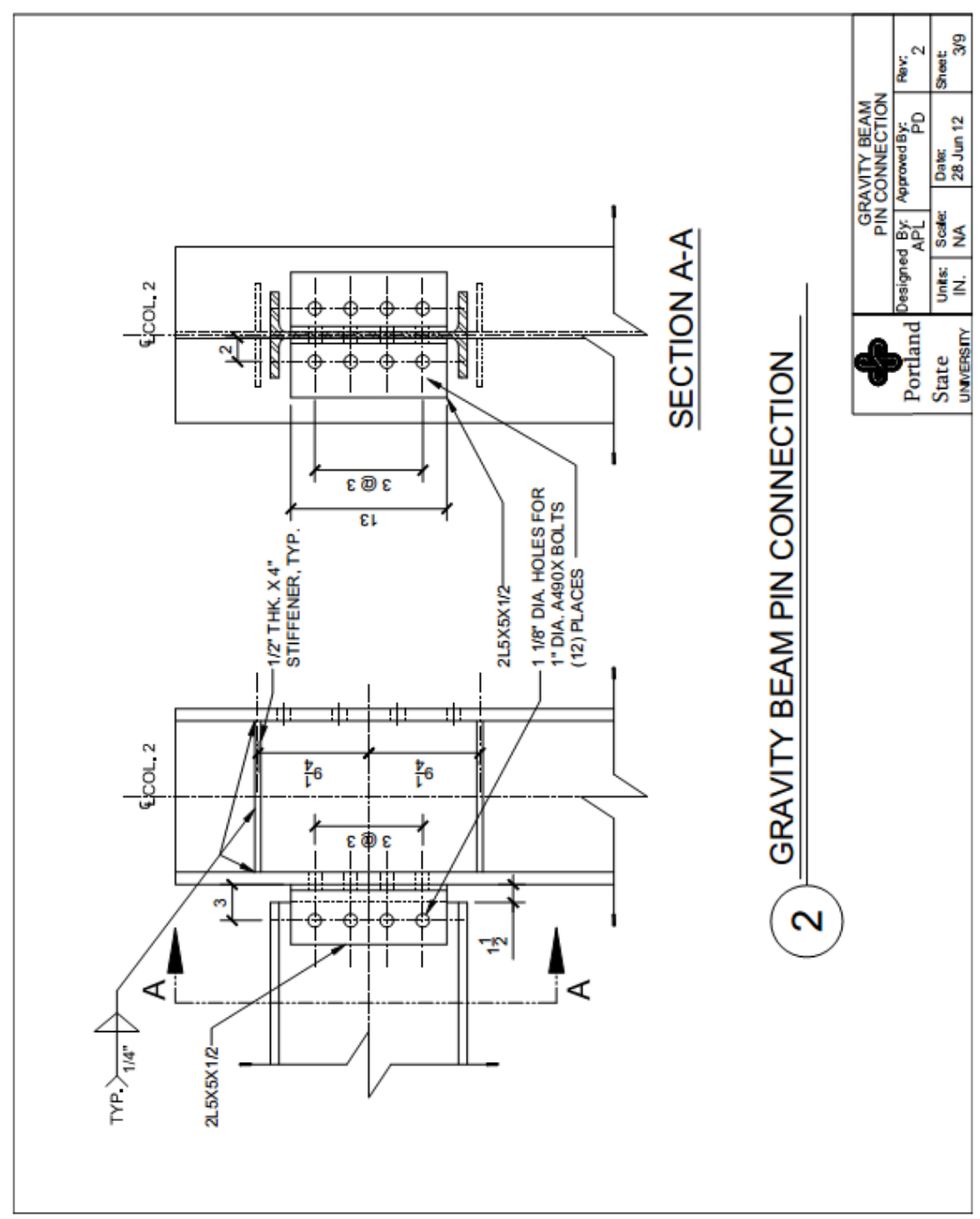




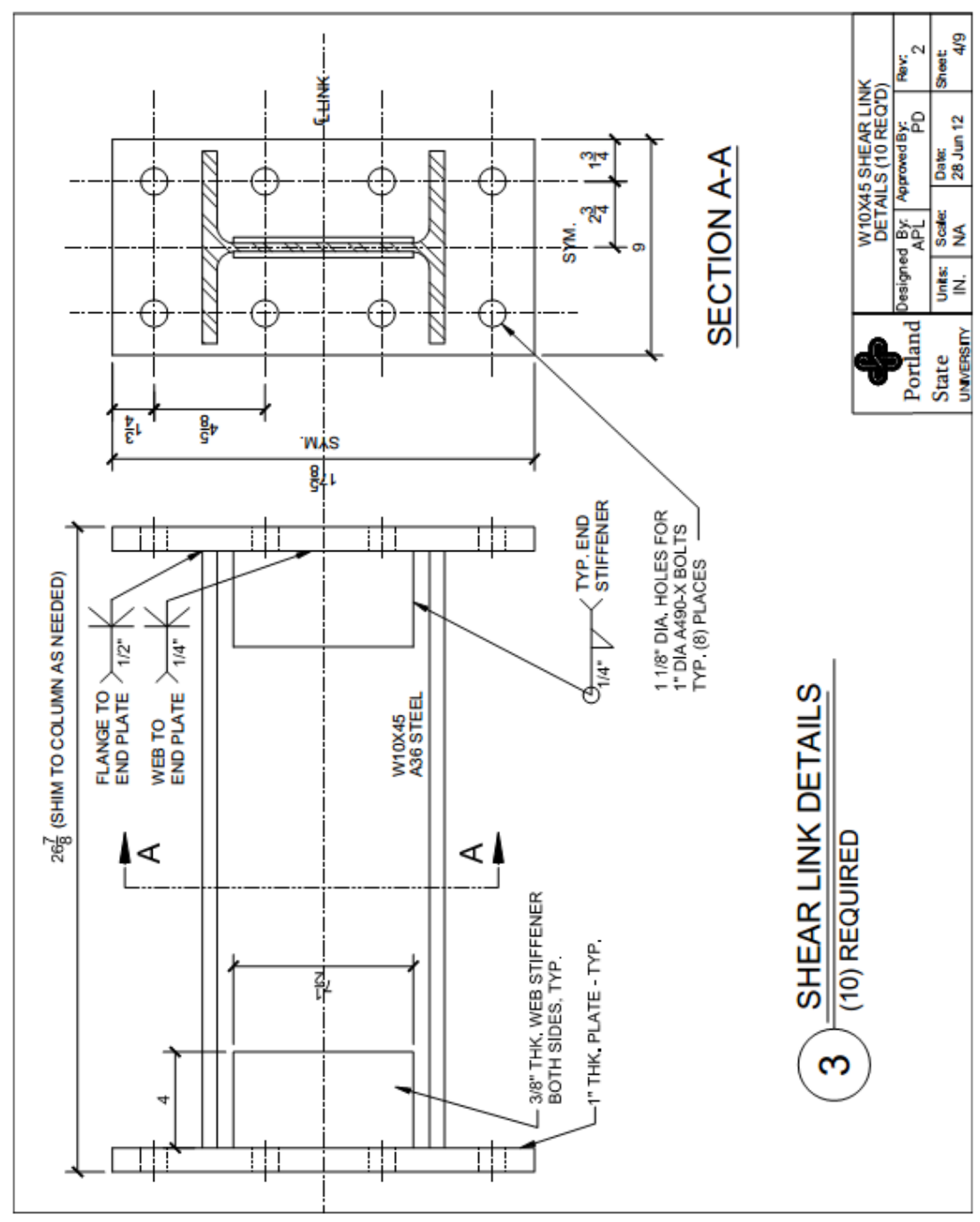




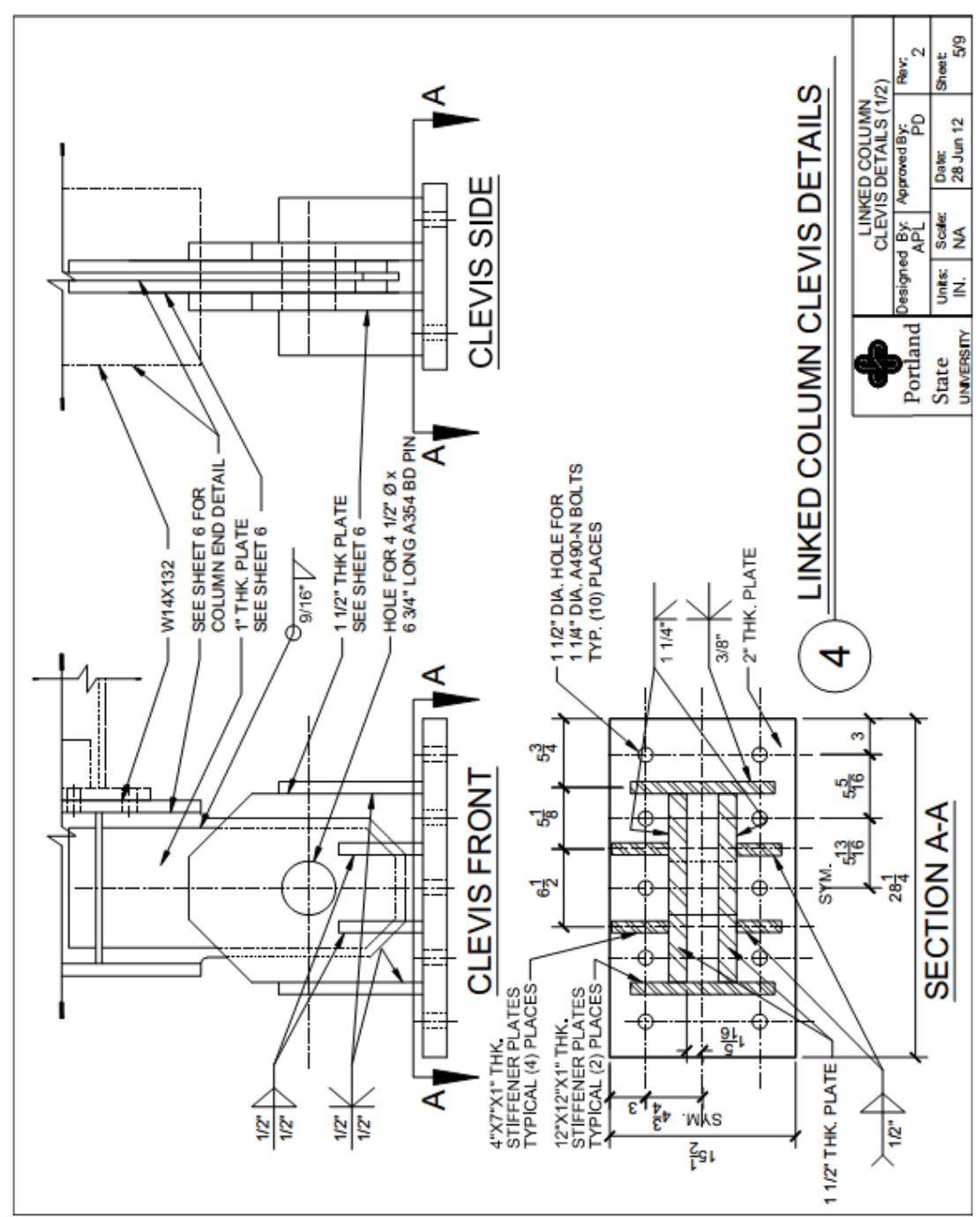




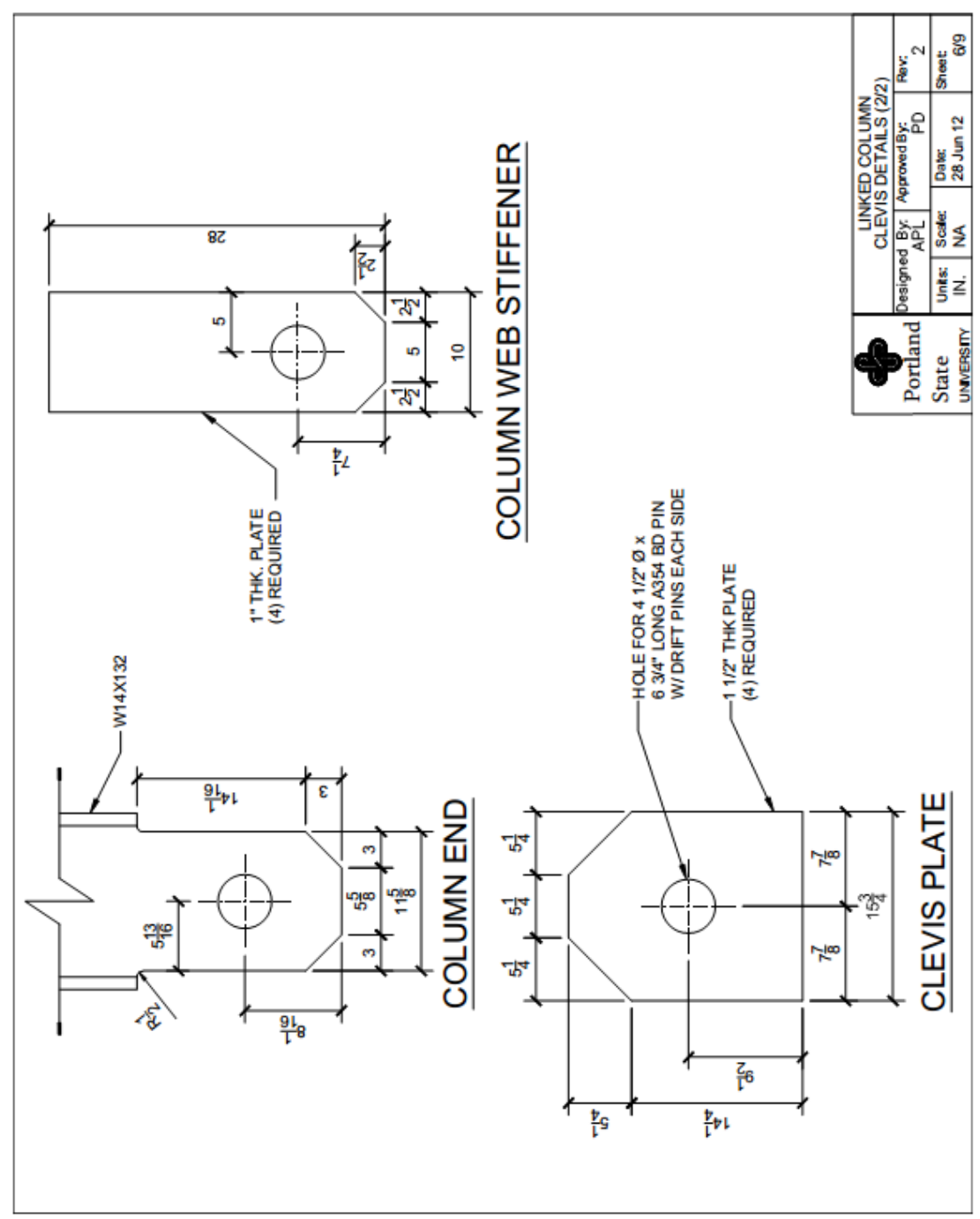




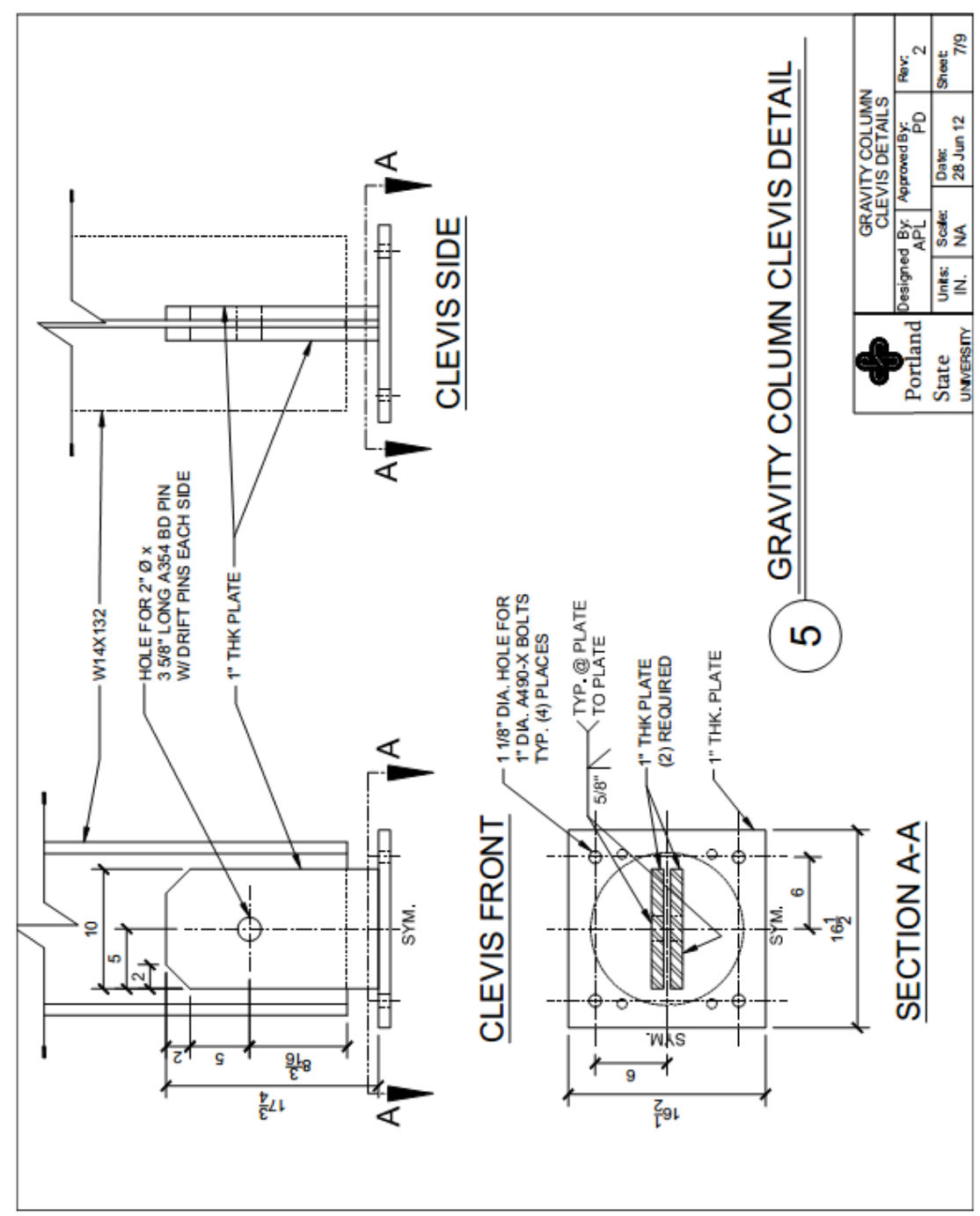




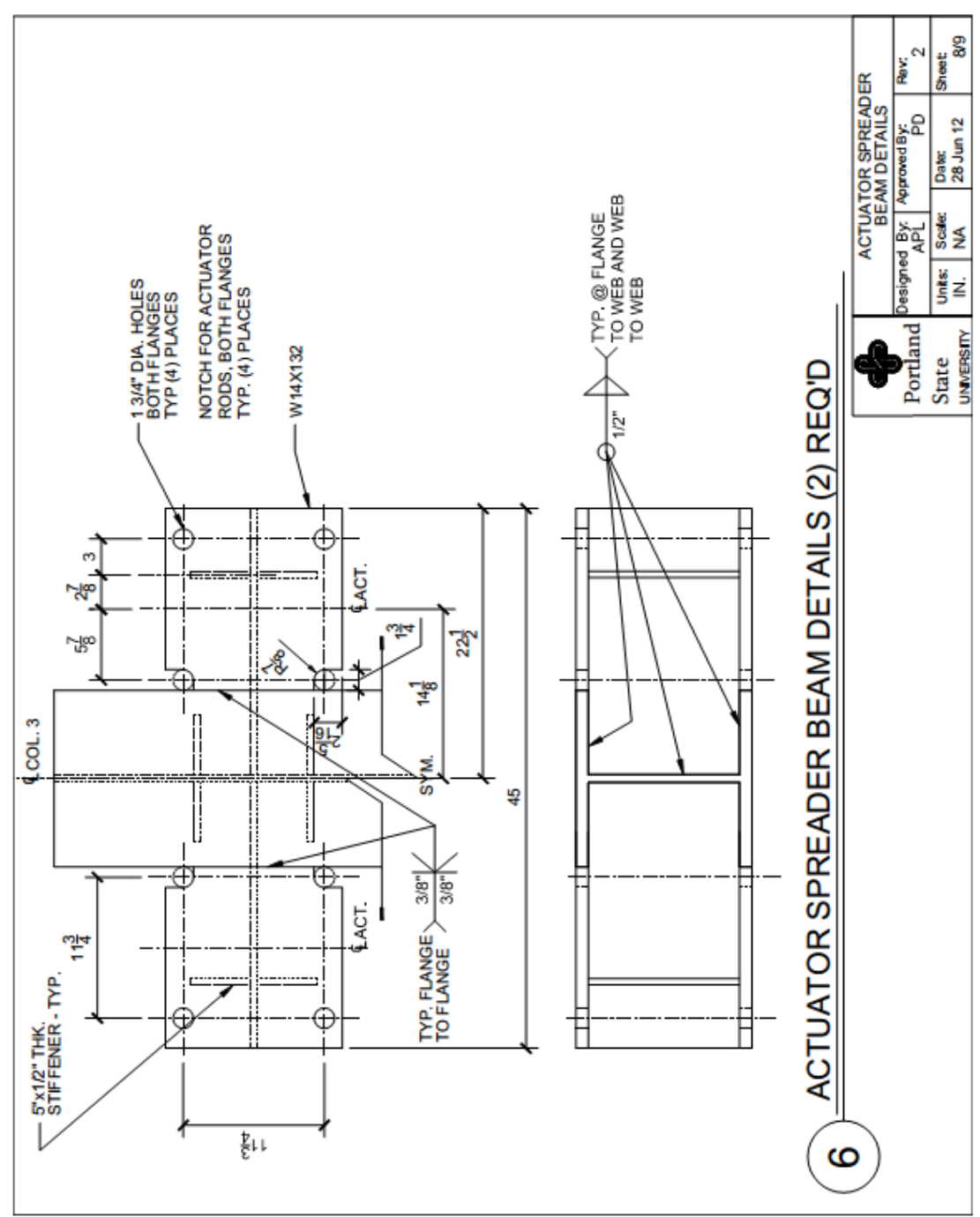




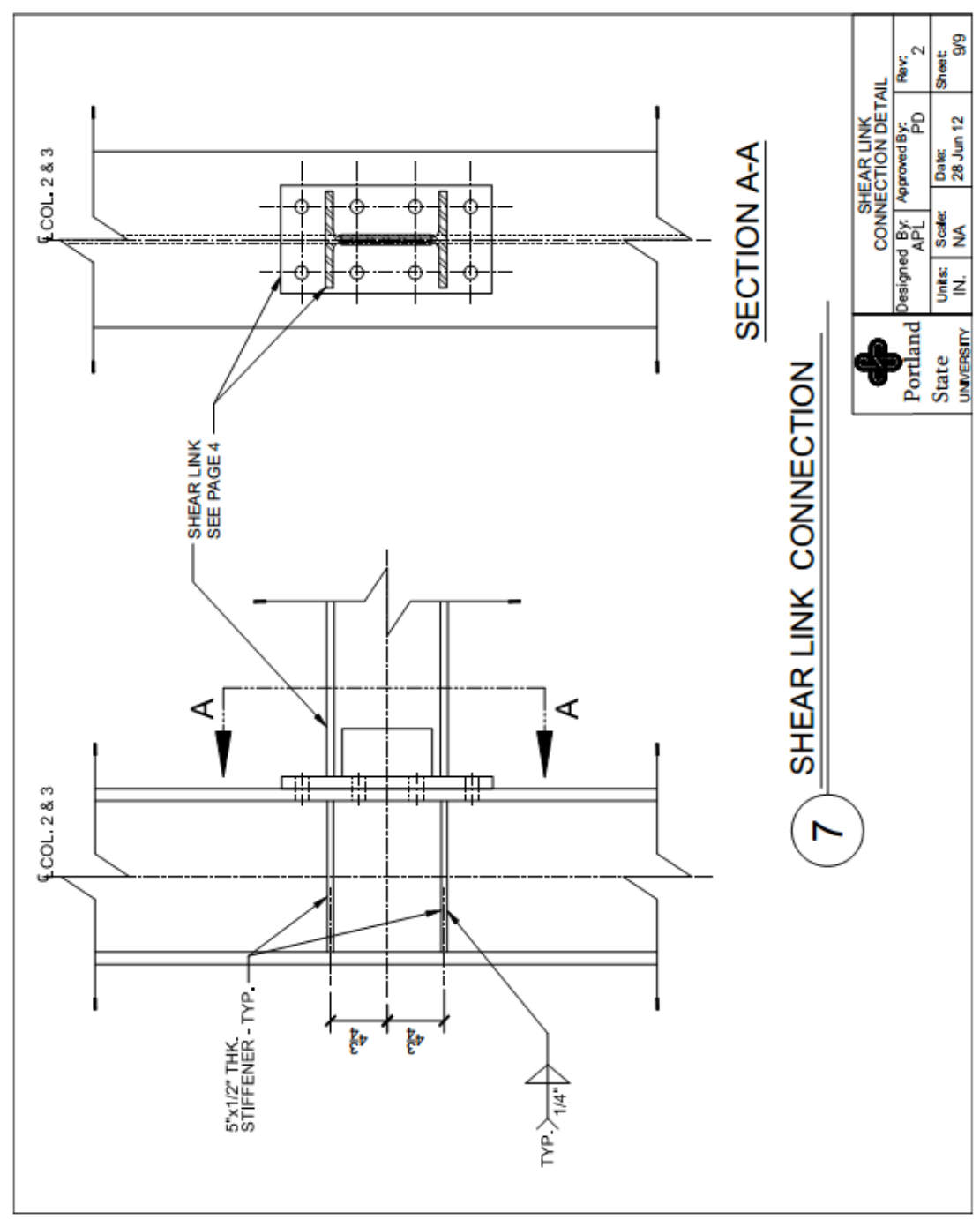


Appendix-C Mill reports 


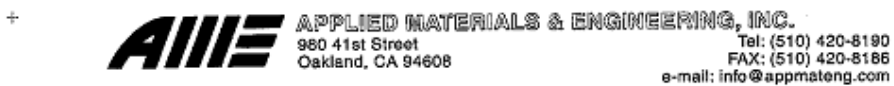

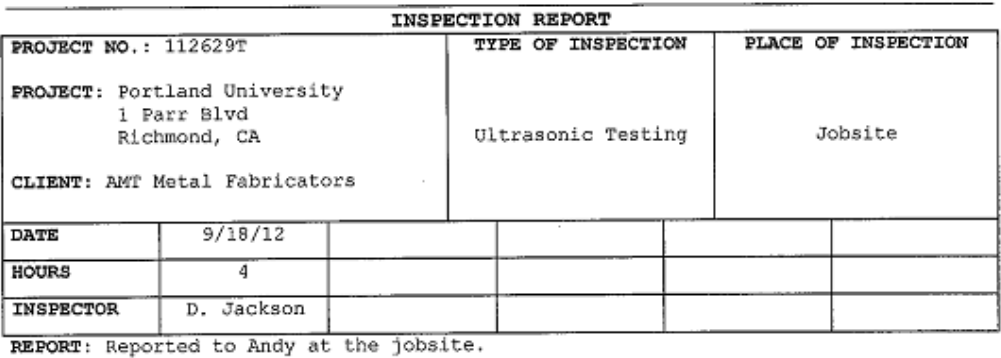

Performed ultrasonic inspection of complete foint penetration groove welds on wide flange beam end connection plates at piece mark M7 (2).

A total of 4 welds inspected with no rejectable indication detected.

Inspection performed as per AwS D1.1.

NOTE: Satisfactory. UT Only.

Work inspected was in compliance with approved job plans and specifications.

Reviewed By:

Dishegant Manomation

Dushyant Manmohan

ec: J. Muela 


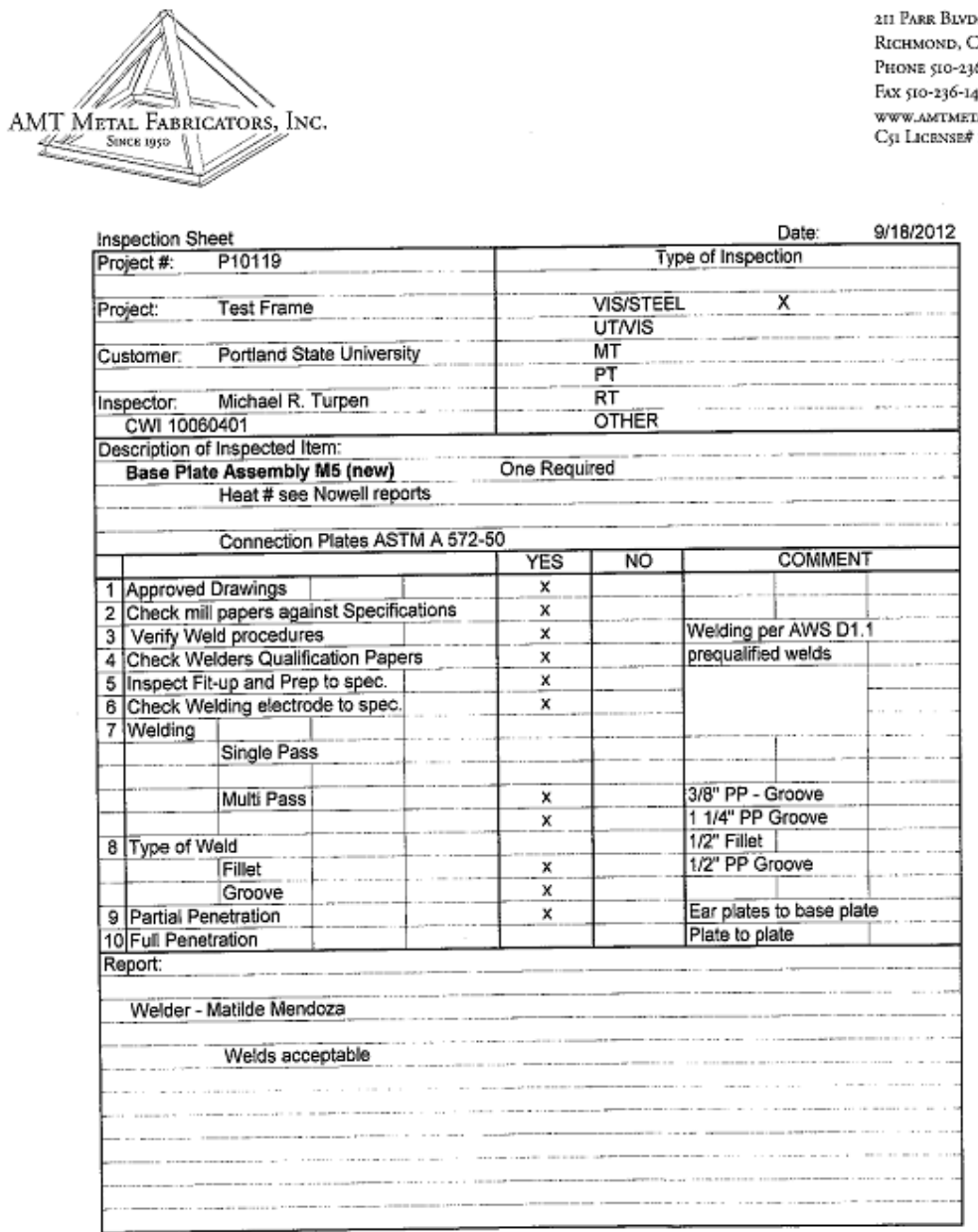

Inspector Michael R Turpen

Michael R Turpen

CWI 1C060401
OC1 EXP. 6/1/2013 


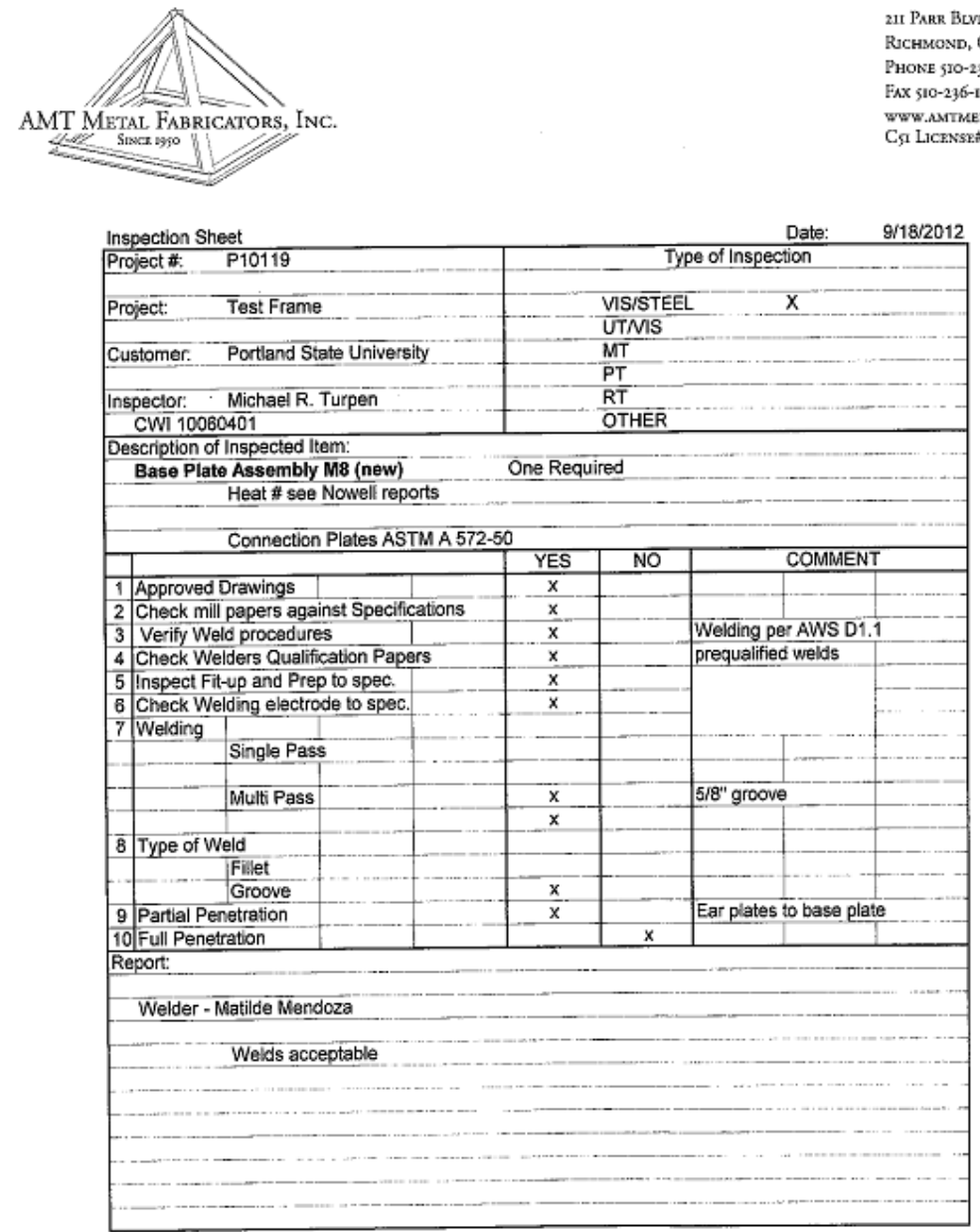

Inspector Michael R Turpen 


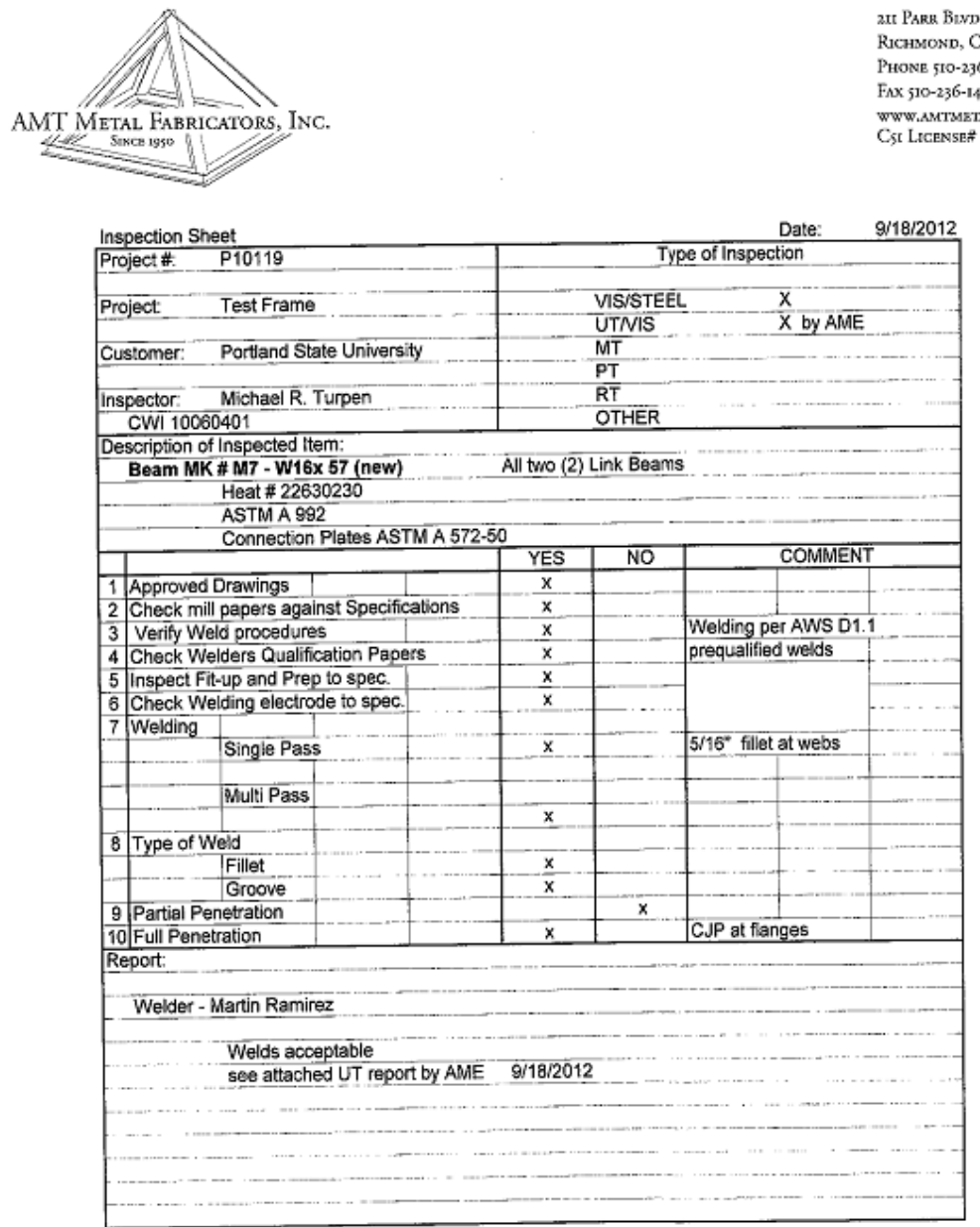

Inspector Michael R Turpen

Michael R Turpe

CWI 10060401

OC1 EXP. 8/1/2013 


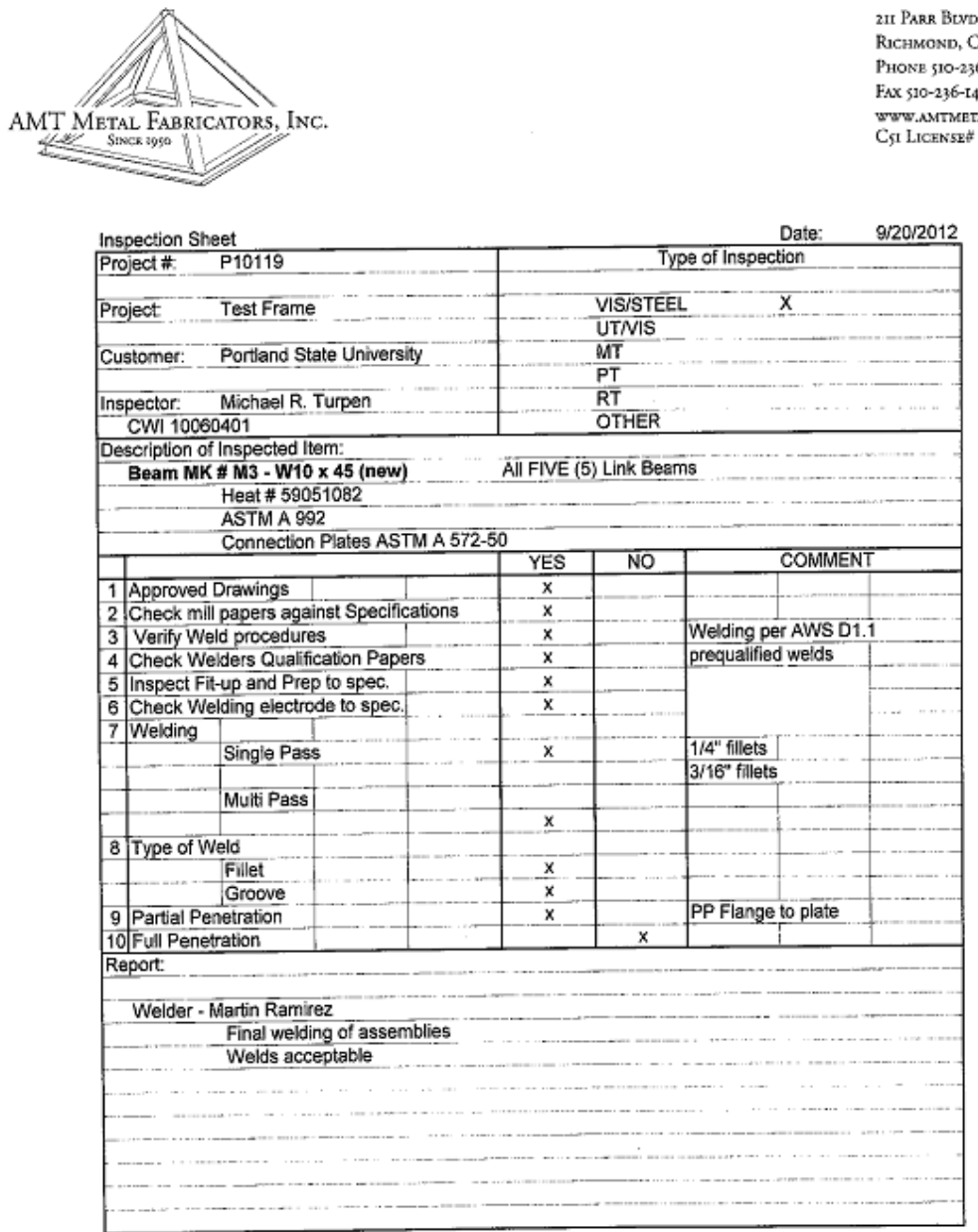

Inspector Michael R Turpen

Michael R Turpen

QC1 EXP. 6/1/2013 


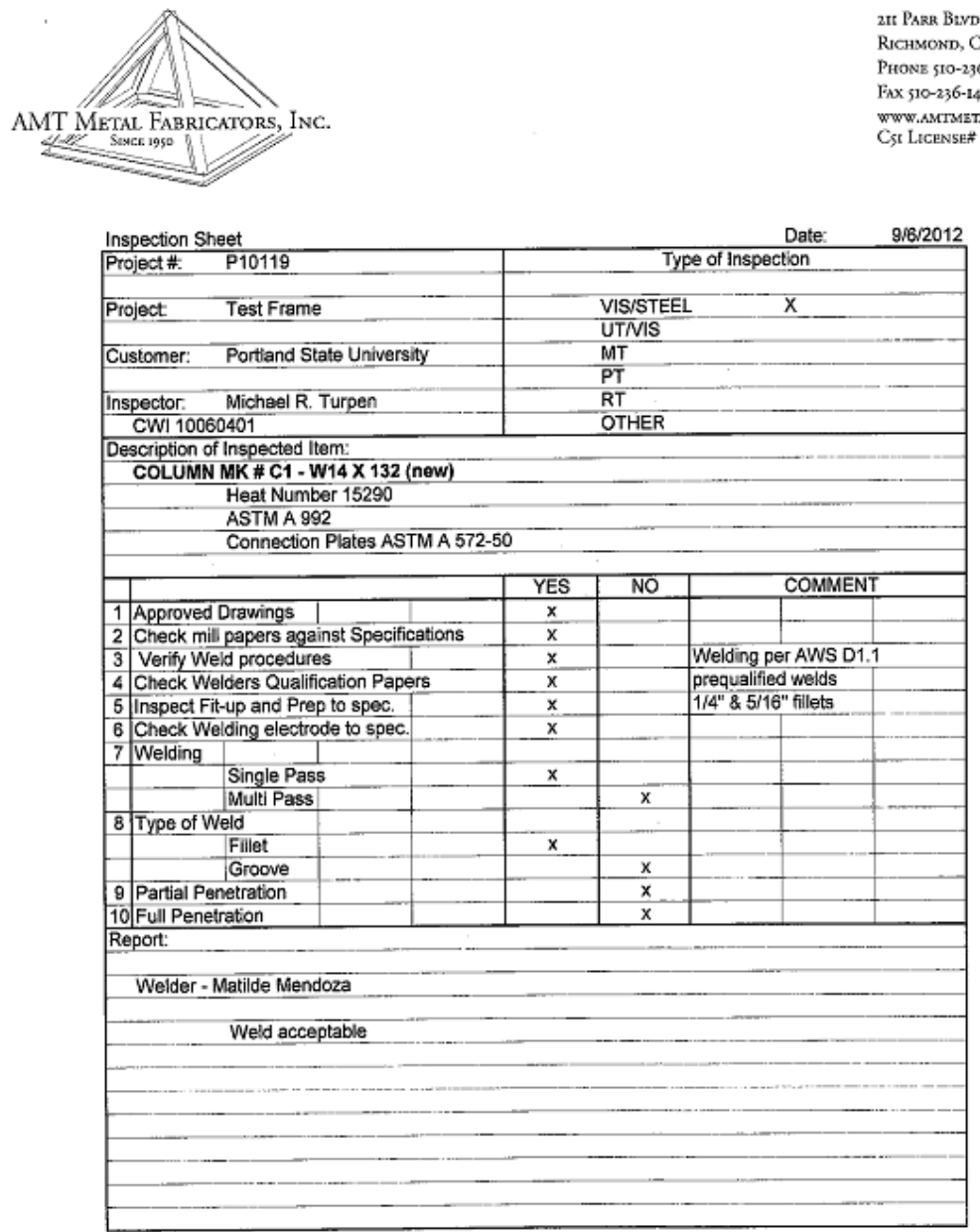

Inspector Michael R Turpen

Michael R Turpen

OC1 EXP 6/1/2013 


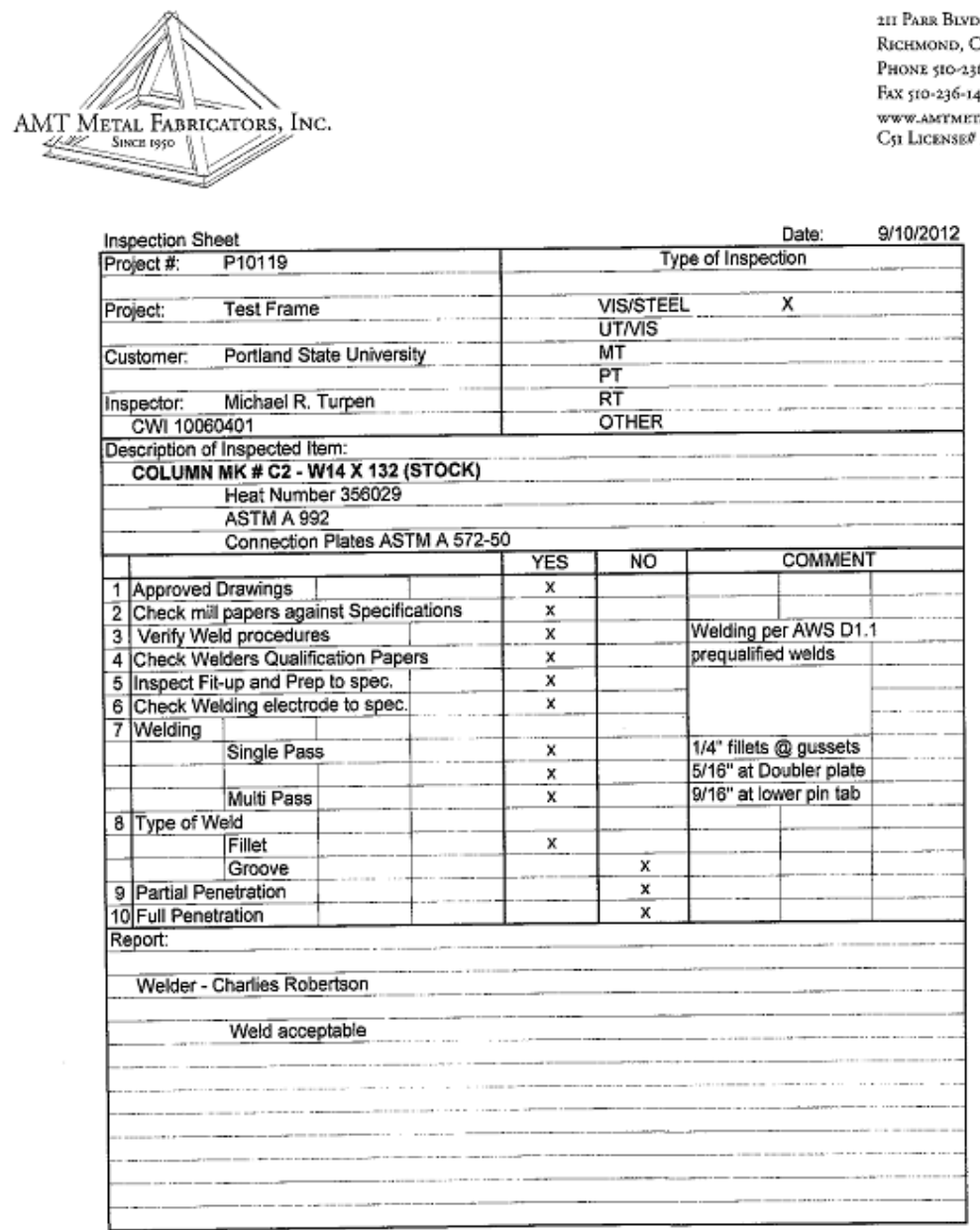

Inspector Michael R Turpen

Michaes R Turpe

QC1 EXP. 6/1/2013 


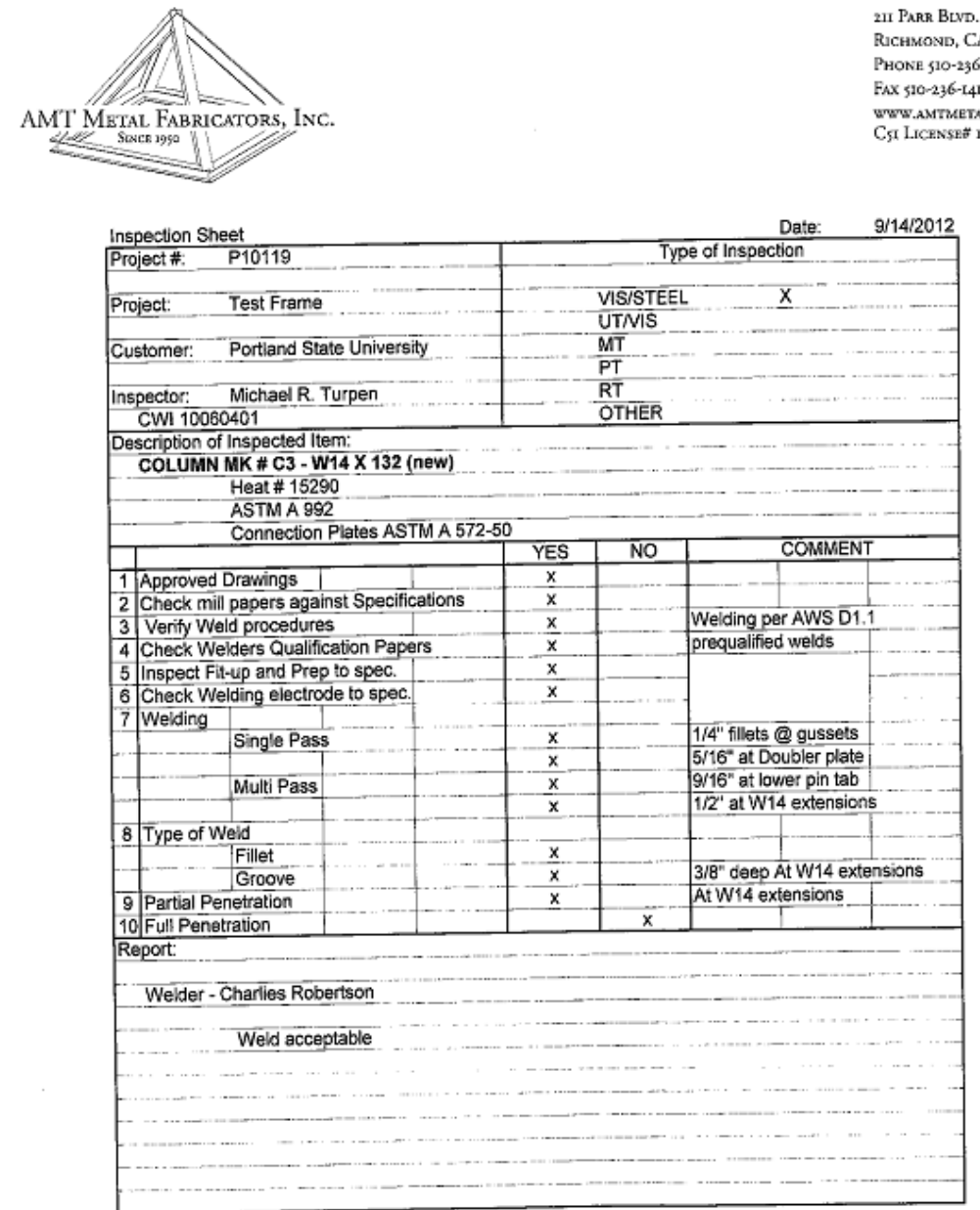

Inspector Michael R Turpen

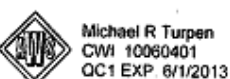

OC1 EXP 6/1/2013 


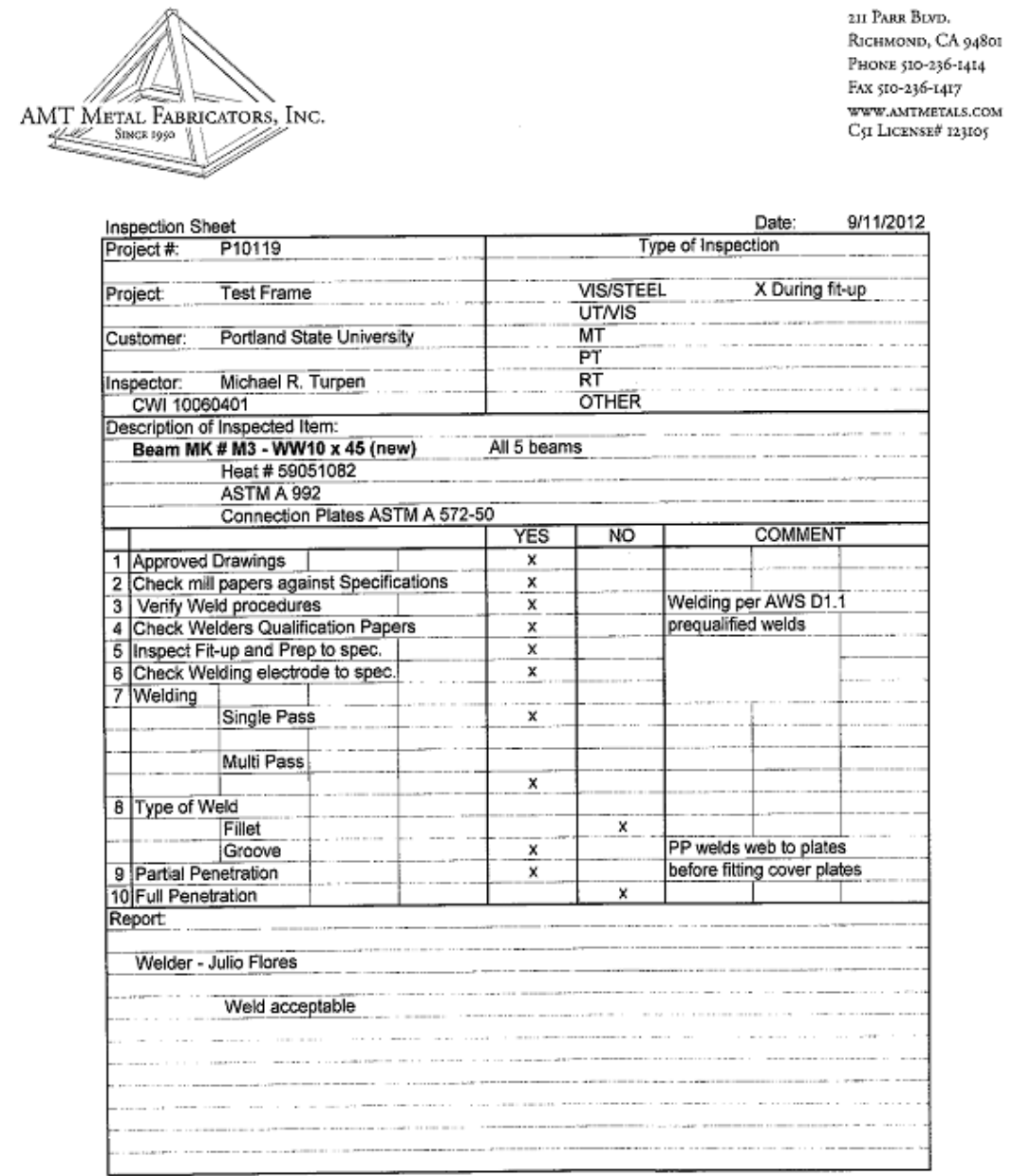

Inspector Michael R Turpen

Michael $R$ Turpen

QC1 EXP. 6/12013 


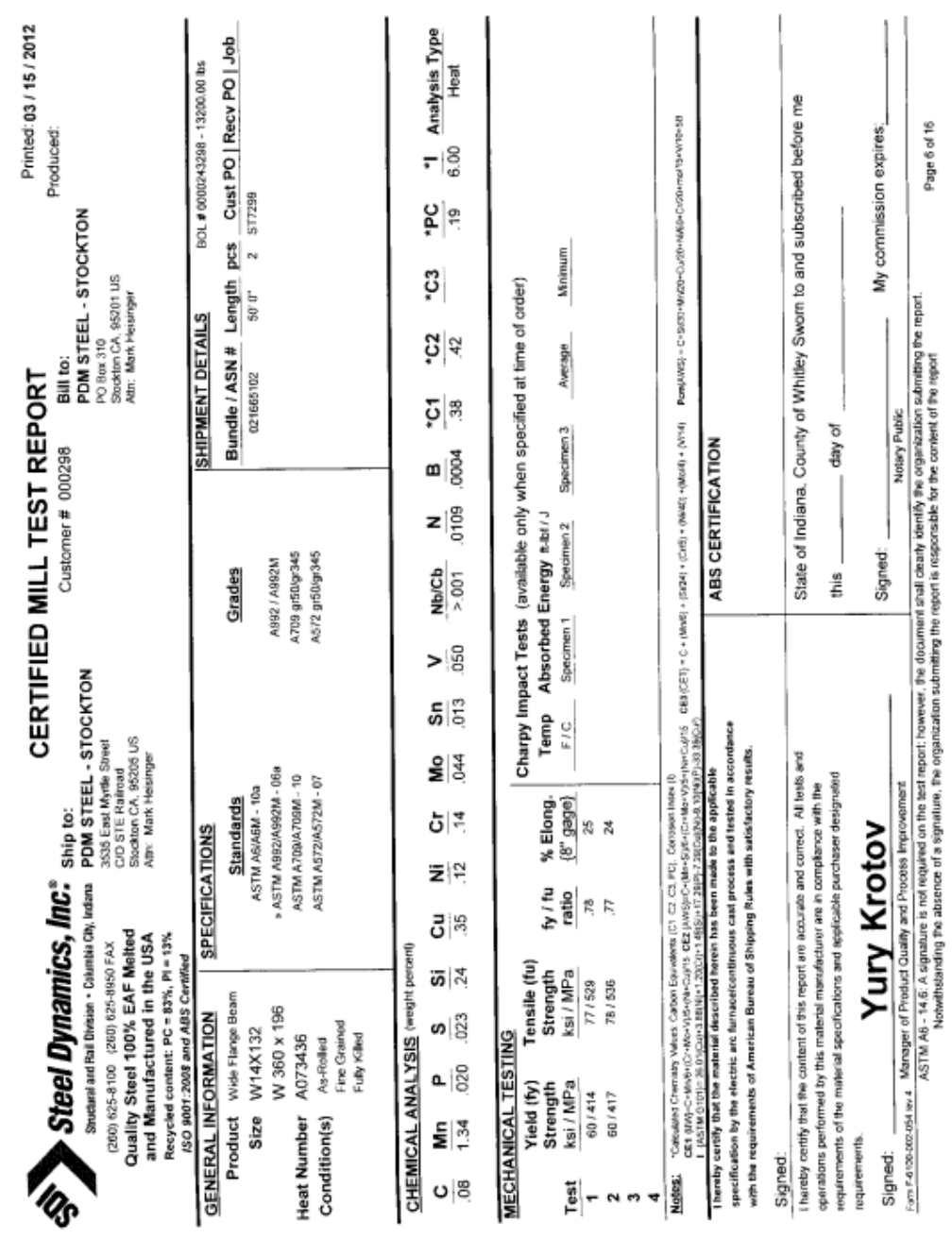



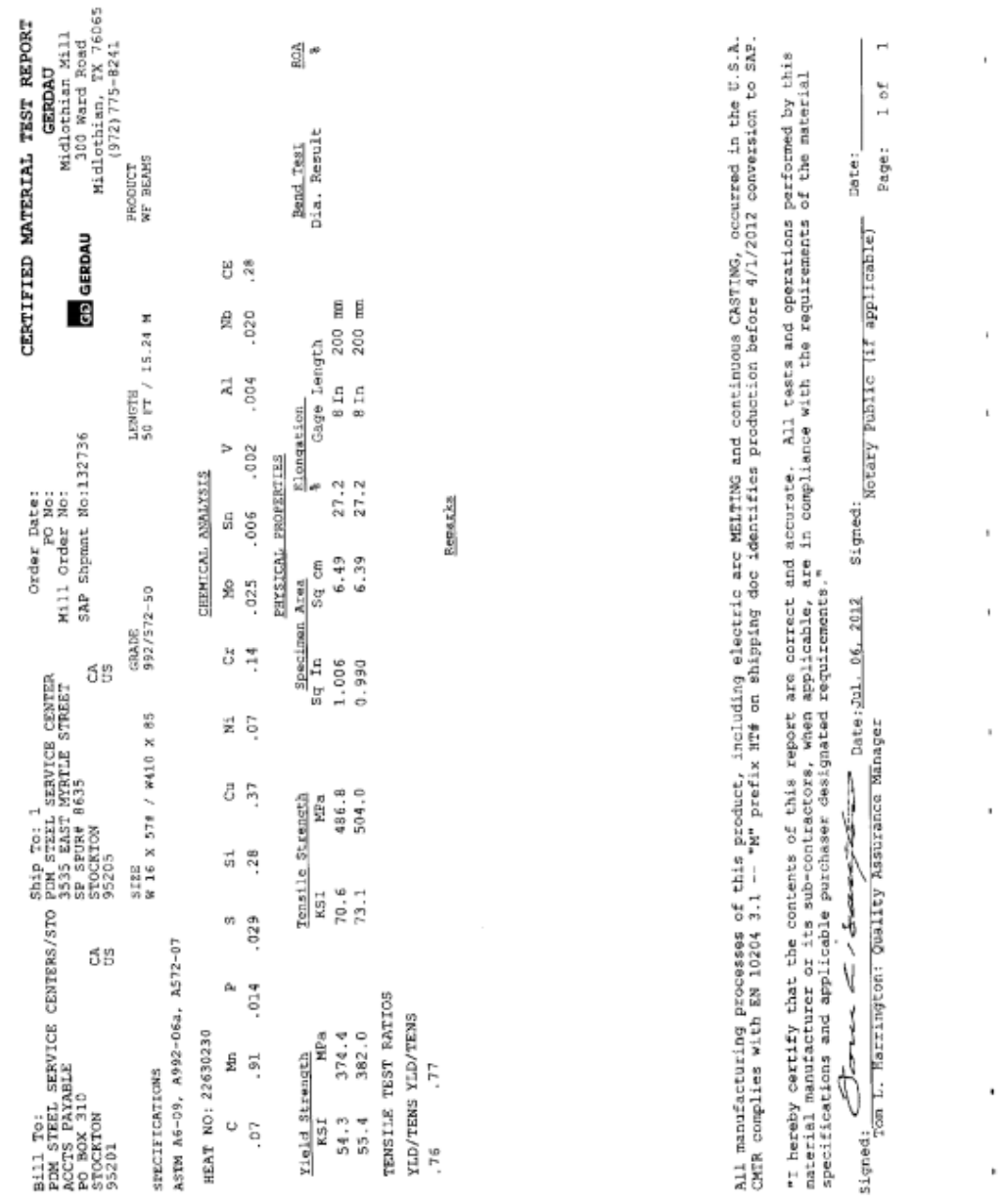

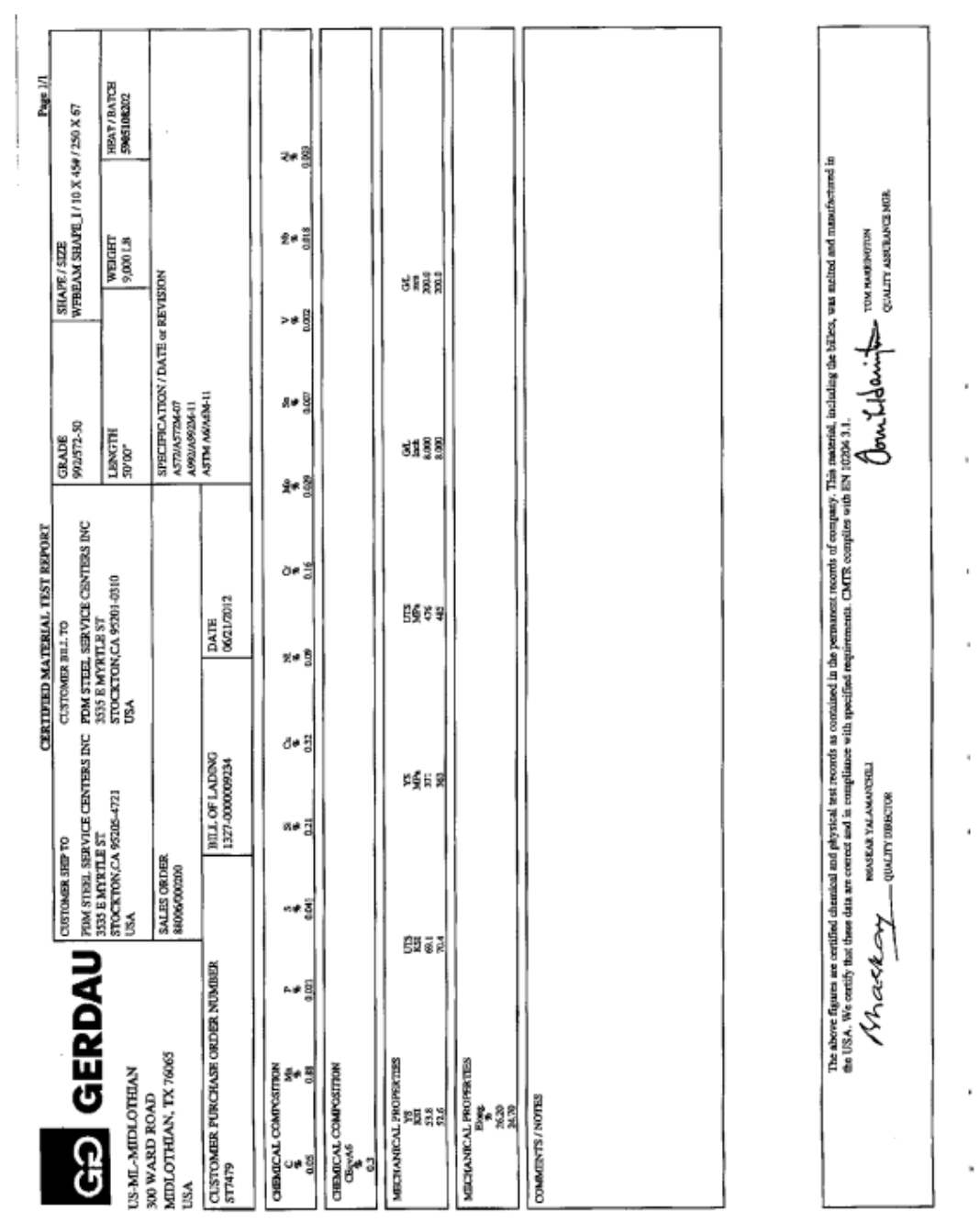